This item was submitted to Loughborough's Research Repository by the author.

Items in Figshare are protected by copyright, with all rights reserved, unless otherwise indicated.

\title{
A STEP-compliant approach to turning operations
}

PLEASE CITE THE PUBLISHED VERSION

PUBLISHER

(c) Yusri Yusof

\section{PUBLISHER STATEMENT}

This work is made available according to the conditions of the Creative Commons Attribution-NonCommercialNoDerivatives 4.0 International (CC BY-NC-ND 4.0) licence. Full details of this licence are available at: https://creativecommons.org/licenses/by-nc-nd/4.0/

\section{LICENCE}

CC BY-NC-ND 4.0

\section{REPOSITORY RECORD}

Yusof, Yusri. 2019. "A Step-compliant Approach to Turning Operations". figshare. https://hdl.handle.net/2134/35164. 
E Loughborough

\section{University Library}

University

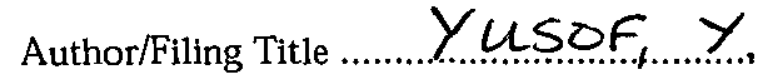

Class Mark ….........................

Please note that fines are charged on ALL overdue items.

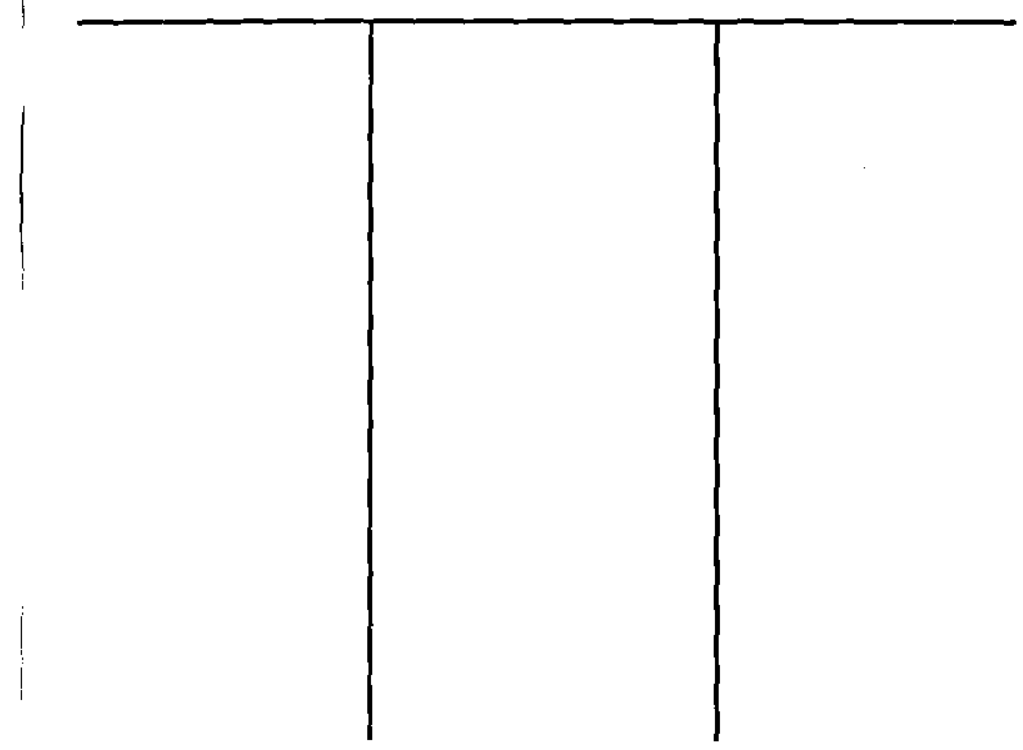




\title{
A STEP-COMPLIANT APPROACH TO TURNING OPERATIONS
}

\author{
By \\ Yusri Yusof
}

A Doctoral Thesis

submitted in partial fulfilment of the requirements

for the award of

Doctor of Philosophy

of Loughborough University

October 2007

Mechanical and Manufacturing Engineering

Loughborough University

(C) Yusri Yusof (2007) 


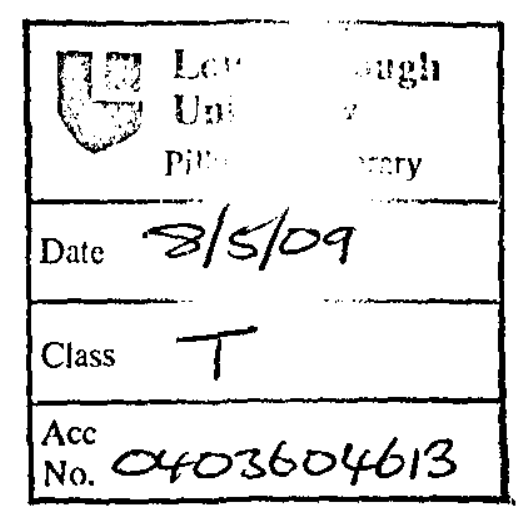




\begin{abstract}
There is no doubt that today manufacturing is more competitive and challenging than ever before in trying to respond to "production on demand". Companies from east and west and all over the world have changing rules of business and need to collaborate beyond geographic boundaries with the support of the rapid advancement of information technology associated with manufacturing technology. To satisfy customers' demands for product variety and the industrial need for high precision, numerically controlled machining with multiple axes and sophisticated machine tools are required. Due to the complexity of programming there is a need to model their process capability to improve the interoperable manufacturing capability of machines such as turning centres.

This thesis focuses on the use of the new standard; ISO 14649 (STEP-NC), to address the process planning and machining of discrete turned components. It explores how ISO 14649 can be used to combine turning and milling operations to support interoperable CNC manufacturing of rotational asymmetric components at a single turning centre. The STEP-NC standard presents the opportunity to develop a new structure for feature-based process planning to address the process planning and machining of discrete components. This research proposes a STEP Compliant NC structure for generation of ISO 14646 code which can be used for turned component manufacture.

The major contribution of this research is the creation of a computational environment for a STEP-NC compliant system for turning operations namely SCSTO. SCSTO is the experimental part of the research, supported by specification of information models and constructed using a structured methodology and object-oriented methods. SCSTO was developed to generate a Part 21 file based on machining features to support the interactive generation of process plans utilising feature extraction. Case study components have been tested to prove that the new approach (STEP-NC) can be used to generate code equivalent to currently used G-codes with some benefits such as the elimination of the post-processor. An important aspect was the need to overcome the complexities of component geometry including milling features so as to have ability to manufacture turn/mill components.
\end{abstract}




\section{Keywords}

STEP-NC, STEP Compliant, ISO 10303, ISO 14649, Rotational Component, Turning Operations and Process Plans 
To my lovely wife, Rosmawati and to my son Muhammad $\mathrm{A}_{\text {iman and }} \mathrm{M}_{\text {uhammad }} \mathrm{A}_{\text {mmar }}$ 


\section{ACKNOWLEDGEMENTS}

Without help from many work colleagues, friends and family, this thesis would not have been possible to accomplish. With that in mind the author would like to thank the following wonderful people. Firstly the I wish to express my sincere gratitude to my supervisor, Professor Keith Case, for his direction, support, patience and friendship. I am sure that would be harder to finish this work without your support. Keith, you are someone who shares my hopes and dreams. Also, to Professor Stephen Newman from University of Bath for your encouragement and interest in this research.

Many thanks to my colleagues in the TW214 room, especially Gareth Coates, Chris Edwards, Theodoros Staikos, Muhammad Abu Bakar, Abeer Pharaon and Dr. Simon Collins, for the conversations and asides help that made each day more enjoyable. I extend my thanks and appreciation to my friends, Masyarakat Melayu Loughborough (MML), Universiti Tun Hussein Onn (UTHM) and to all others who contributed directly or indirectly, to bring out this thesis successfully.

Last but not least, my deepest gratitude goes to my wife, Rosmawati Maarof, for her encouragement and patience for more than three years. Besides being the mother of our two children, Muhammad Aiman and Muhammad Ammar, she has supported me and given me constant encouragement and care. I wish to express my gratitude to my lovely mother Hjh. Che Gayah Salleh my lovely father Hj. Yusof Awang for their unwavering support, pray and encouragement throughout the period of my research study.

I am really indebted to my family and my parents for their love, cheerfulness, and their understanding of my not being able to spend a lot of time with them, especially during the final stage of this research. Without their sacrifice and support, it would not have been possible for me to complete my thesis.

The author would also like to thank anybody involved directly or indirectly with my research project. 


\section{ABBREVIATIONS}

AIM Application Interpreted Model

ABS Abstract Super-type

AP Application Protocols

API Application Programming Interface

APT Automatically Programmed Tool

ARM Application Reference Model

CAD Computer Aided Design

CAM Computer Aided Manufacturing

CAPP Computer Aided Process Planning

$\mathrm{CAx} \mathrm{CAD} / \mathrm{CAPP} / \mathrm{CAE} / \mathrm{CAM} /$ (the application of computer aided methods to the product lifecycle)

$\mathrm{CD}^{`} \quad$ Committee Draft

CNC Computer Numerical Control

DBMS Database Management Systems

DIS Draft International Standard

ESPRIT European Strategies Programme For Research Into Information Technology

FAPT Fanuc Automatically Programmed Tools

FDIS Final Draft International Standard

IDEF0 ICAM Definition Level 0

ICAM Integrated CAM

IGES Initial Graphics Exchange Specification

IMS Intelligent Manufacturing System

IP3AC Integrated Platform for Process Planning and Control

IR Integrated Resource

IS International Standard

ISO International Organisation for Standardisation

ISO/TC184 Technical Committee 184, Industrial Automation Systems and Integration JVM Java Virtual Machine 


$\begin{array}{ll}\text { MDM } & \text { Manufacturing Data Model } \\ \text { NC } & \text { Numerical Control } \\ \text { NRL-SNT } & \text { National Research Laboratory for STEP-NC Technology } \\ \text { OMAC } & \text { Open Modular Architecture Controller } \\ \text { PDDI } & \text { Product Definition Data Interface } \\ \text { PDES } & \text { Product Data Exchange Specification } \\ \text { PDM } & \text { Product Data Model } \\ \text { RAMP } & \text { Rapid Acquisition of Manufactured Parts } \\ \text { SC1 } & \text { Subcommittee 1, Physical Data Control } \\ \text { SC4 } & \text { Subcommittee 4, Industrial Data } \\ \text { SCSTO } & \text { STEP Compliant System for Turning Operations } \\ \text { SDAI } & \text { Standard Data Access Interface } \\ \text { SDK } & \text { Software Development Kit } \\ \text { SET } & \text { Standard d'Echange et de Transfert } \\ \text { SFP } & \text { Shopfloor -oriented NC Programming } \\ \text { STEP } & \text { Standard for the Transfer and Exchange of Product Model Data } \\ \text { TC184/CS1 } & \text { Technical Committee 184/Subcommittee 1 } \\ \text { TC184/CS4 } & \text { Technical Committee 184/Subcommittee 4 } \\ \text { TFL } & \text { Turning Feature Library } \\ \text { TS } & \text { Technical Specification } \\ \text { UML } & \text { Unified Modelling Language } \\ \text { VDA/FS } & \text { Verdand des Automobilindustrie Flachen Schnittstelle. A German } \\ \text { WG7 } & \text { extension of IGES for the transfer of surface data. } \\ \text { XML } & \text { Work Group 7 of Subcommittee 1, ISO/TC184 } \\ & \\ \text { Extensible Markup Language } \\ \text { ST }\end{array}$




\section{TABLE OF CONTENTS}

ABSTRACT .......................................................................................................III

ACKNOWLEDGEMENTS ................................................................................................... VI

ABBREVIATIONS ...................................................................................................... VII

CHAPTER 1 - INTRODUCTION ........................................................................ 1

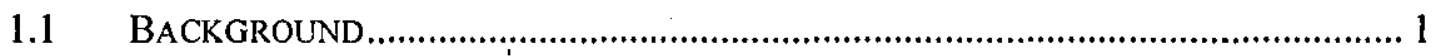

1.2 STANDARD PRODUCT DATA EXCHANGE ........................................................... 2

1.3 STEP-NC ENVIRONMENT FOR MANUFACTURING........................................... 2

1.4 OVERALL RESEARCH INTENTION ................................................................. 5

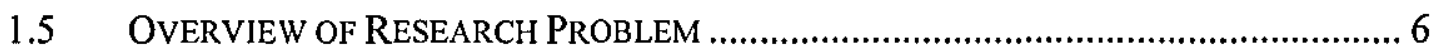

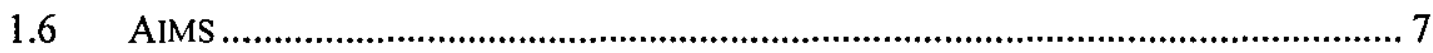

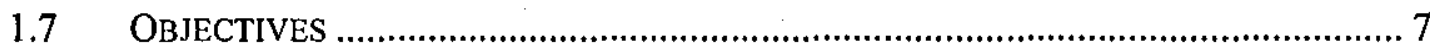

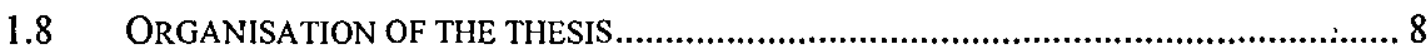

CHAPTER 2 - PRODUCT DATA MODEL AND RELATED STANDARDS ......... 11

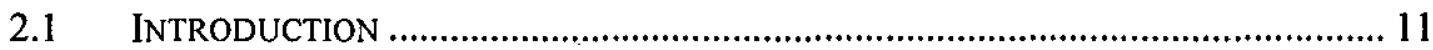

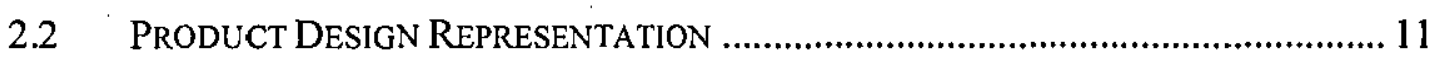

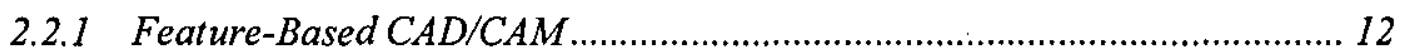

2.2.2 Feature Research Related to Rotational Parts........................................... 14

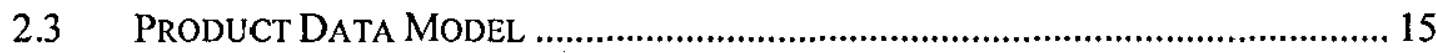

2.3.1 Drawing Transfer File (DXF).......................................................... 17

2.3.2 Initial Graphics Exchange Standard (IGES) ............................................. 17

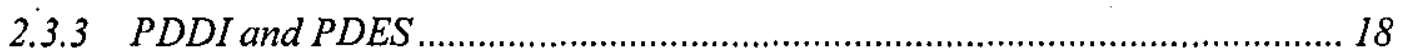

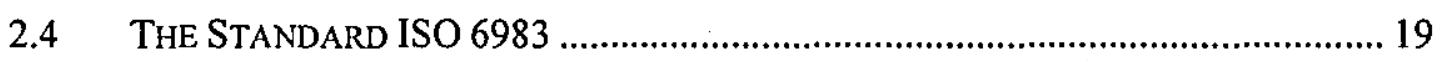

2.5 Standard For the EXChaNGE OF Product Model Data (STEP)................. 21

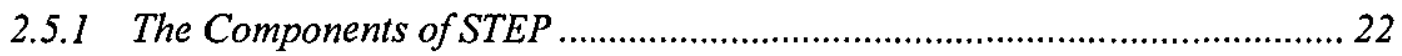

2.5.2 STEP Data Model Structure ………………………….......................... 23

2.5.3 Express Language ............................................................................... 24 
2.6 STEP-NC COMPLIANT MAChINING Process PLANNING ................................. 25

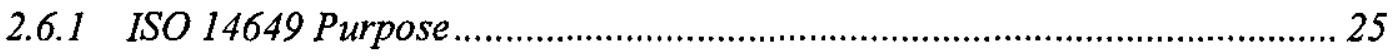

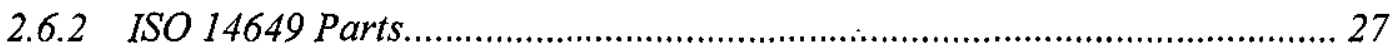

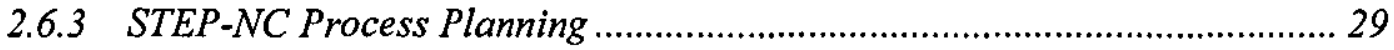

2.6.4 STEP-NC Compliant CAD/CAM System ................................................. 30

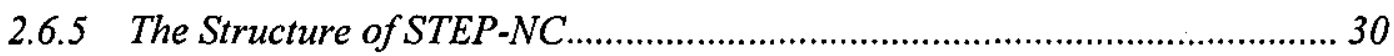

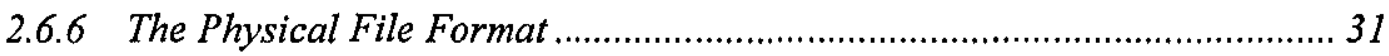

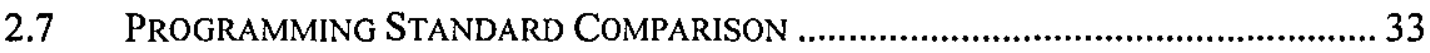

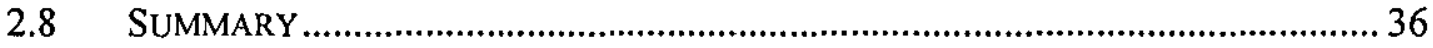

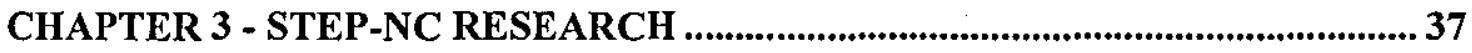

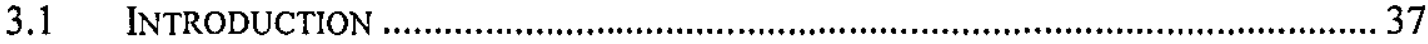

3.2 STEP-COMPLIANT NC: RESEARCH PROJECTS ..................................................... 37

3.3.1 The Intelligent Manufacturing System (IMS)............................................. 38

3.3.2 Intelligent Manufacture for STEP-NC Compliant Machining and Inspection. 40

3.4 STEP-COMPLIANT NC: RESEARCH AND DEVELOPMENT SYSTEMS.................... 41

3.4.1 Review of STEP-Compliant Manufacturing for Milling Machining............ 43

3.4.2 Review of STEP-Compliant Manufacturing for Turning ........................... 44

3.5 REVIEW OF STEP-COMPLIANT SYSTEMS ............................................................ 45

3.5.1 Shop-Floor Programming System (SFPS) ............................................. 47

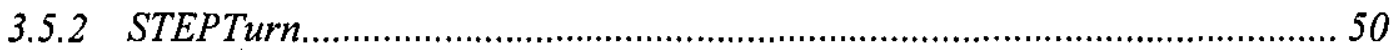

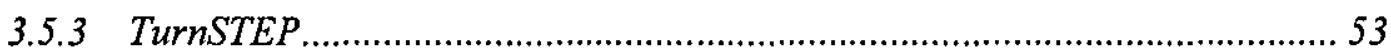

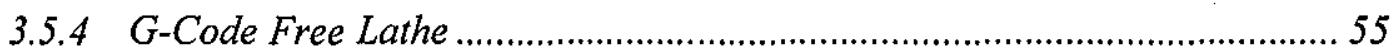

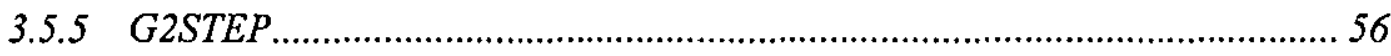

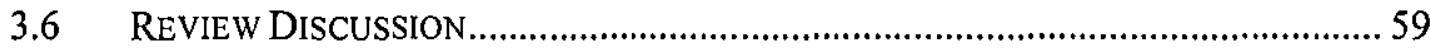

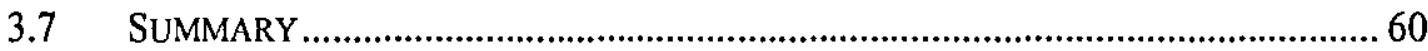

CHAPTER 4 - DESIGN OF THE STEP-NC COMPLIANT SYSTEM

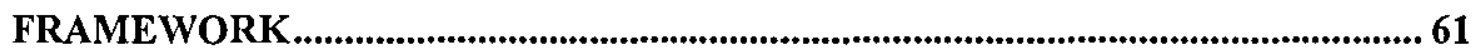

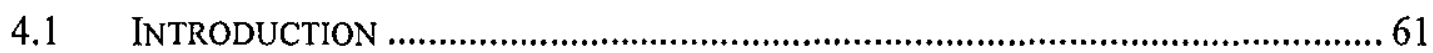

4.2 Fundamentals of MachINING Process PlanNING .................................... 61 
4.3 DESIGN OF A STEP COMPLIANT SYSTEM FOR TURNING OPERATIONS (SCSTO) 62

4.4 INTEROPERABLE MANUFACTURING ENVIRONMENT .......................................66

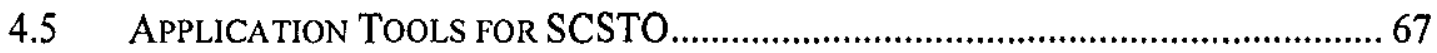

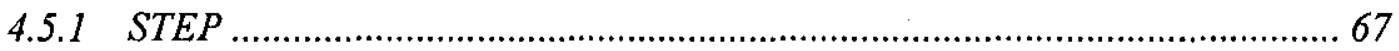

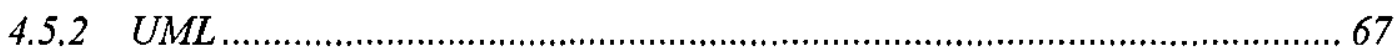

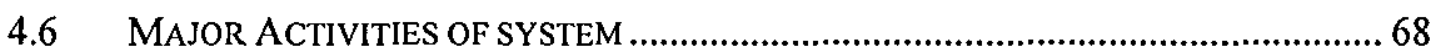

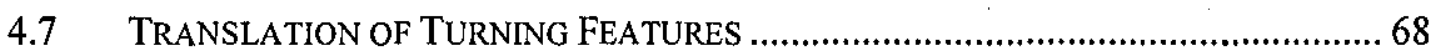

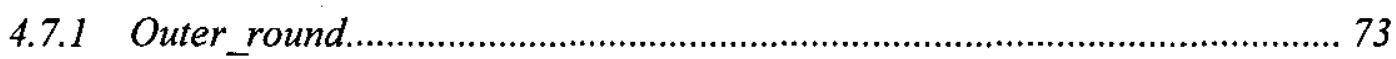

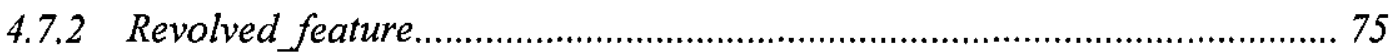

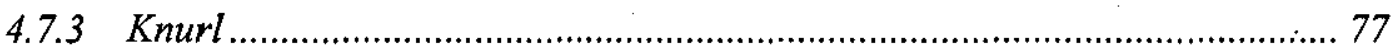

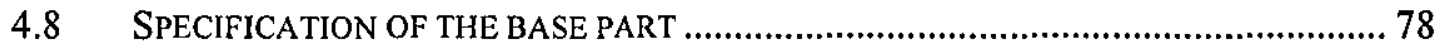

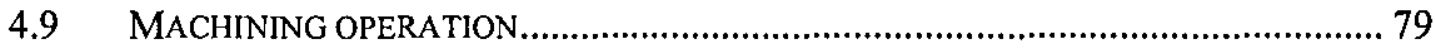

4.9.1 Workpiece and Manufacturing Features for Turning................................ 80

4.9.2 Turning Machining Strategy .............................................................. 81

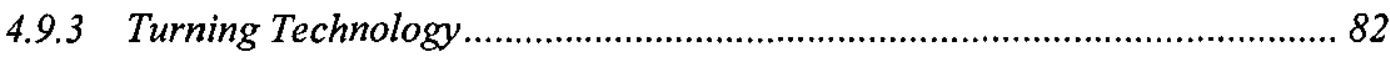

4.9.4 Turning Machine Function ................................................................... 83

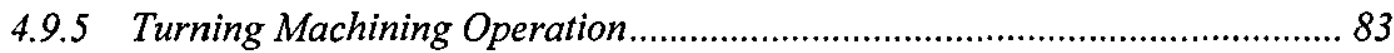

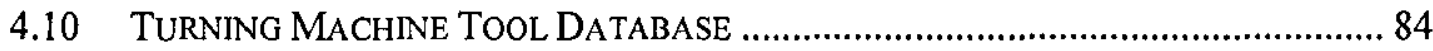

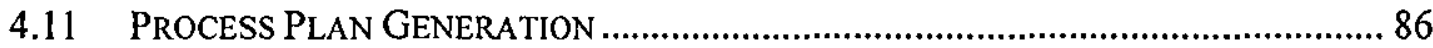

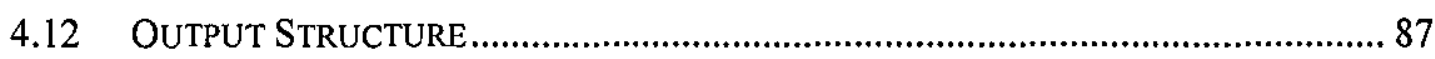

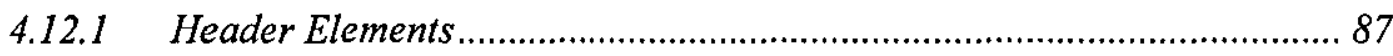

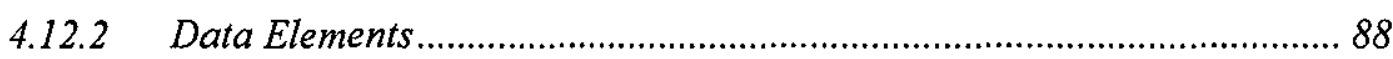

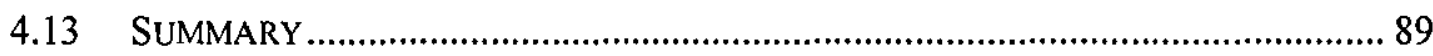

CHAPTER 5 - STEP-NC COMPLIANT INFORMATION MODELLING .............90 90

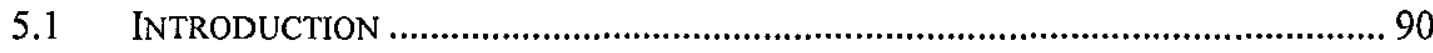

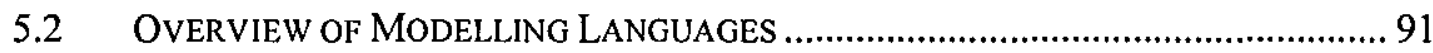

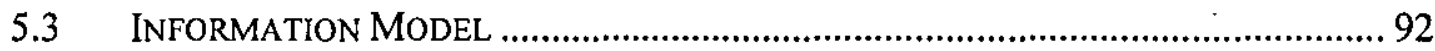

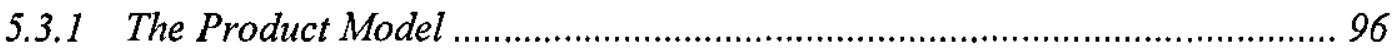

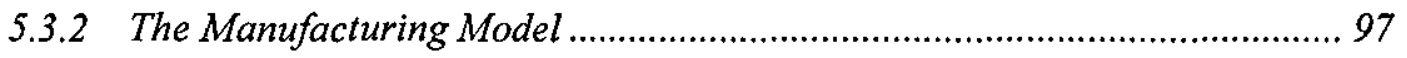

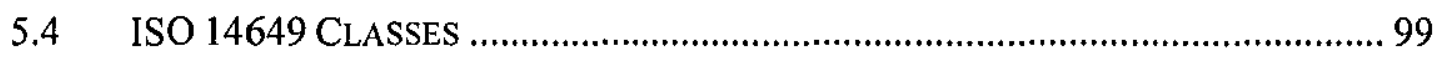

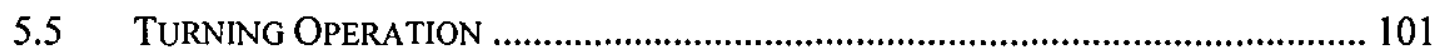




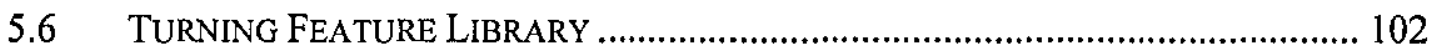

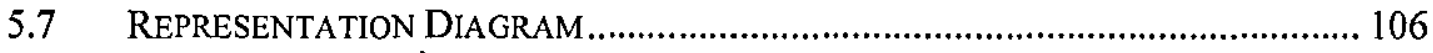

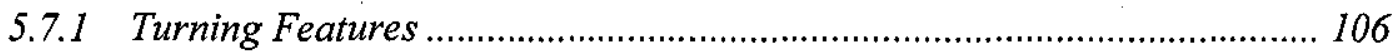

5.7.2 Turning Centre Classification........................................................... 108

5.7.3 Machining Operations ................................................................... 110

5.7.4 Manufacturing Strategy ................................................................ 110

5.7.5 Manufacturing Resources................................................................. 112

5.7.6 The UML Diagram for SCSTO ......................................................... 113

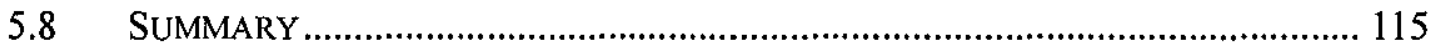

\section{CHAPTER 6 - REALISATION OF A STEP COMPLIANT SYSTEM FOR}

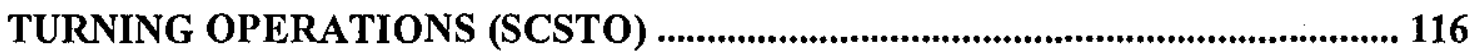

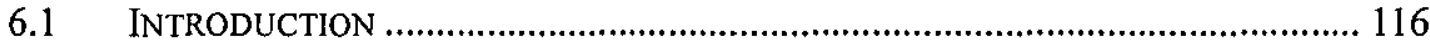

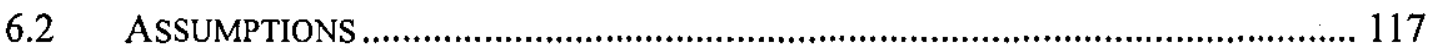

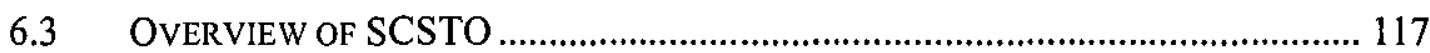

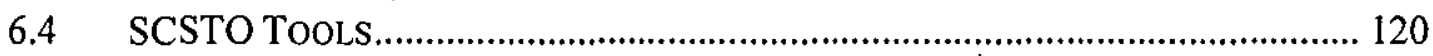

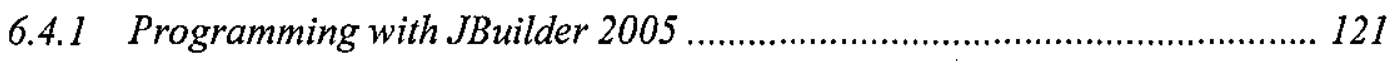

6.4.2 Creating ISO 14649 Classes.................................................................. 122

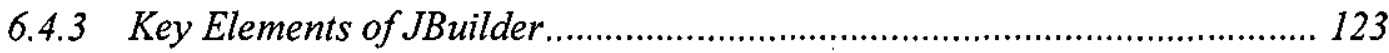

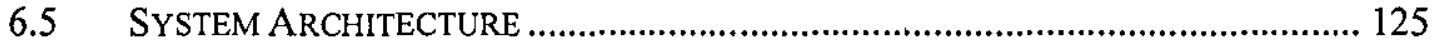

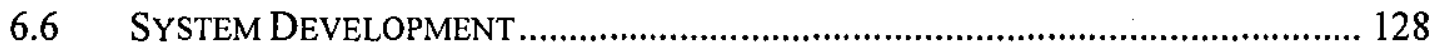

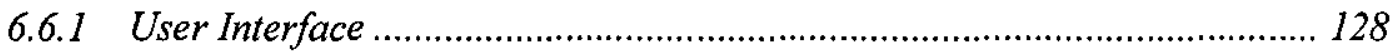

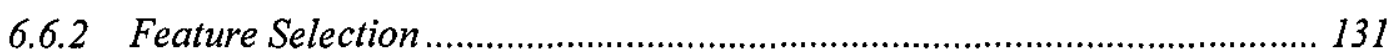

6.6.3 Turning Machining Operations ........................................................ 132

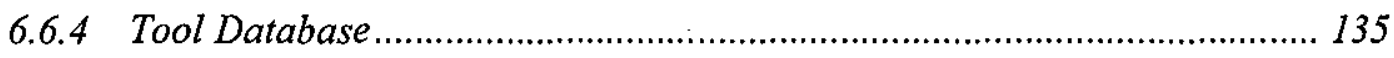

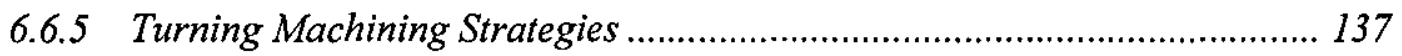

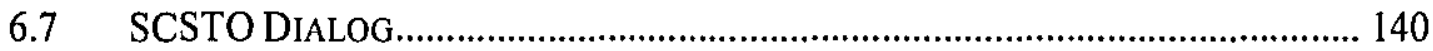

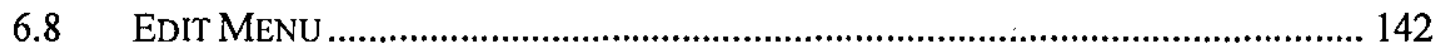

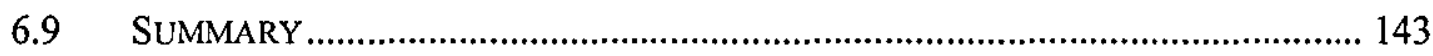

CHAPTER 7 - CASE STUDY IMPLEMENTATION ........................................... 144

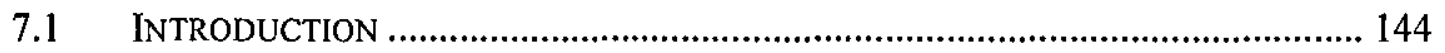




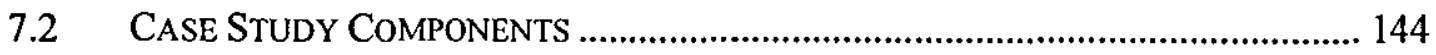

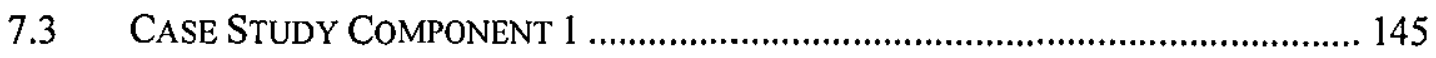

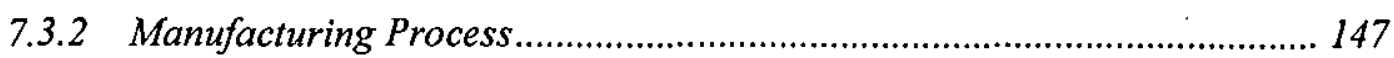

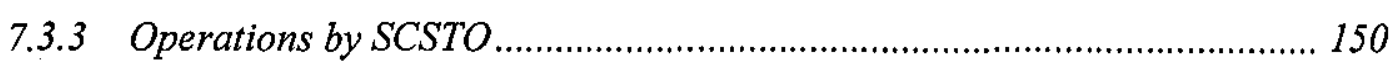

7.3.4 STEP Physical File Created by SCSTO.................................................... 161

7.3.5 NC Programming for Case Study Component 1..................................... 162

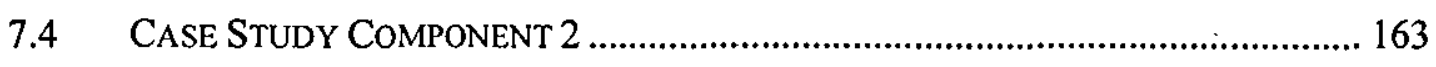

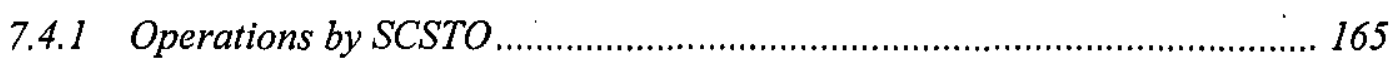

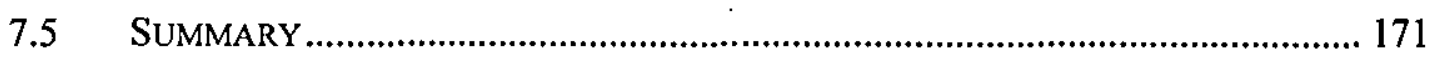

CHAPTER 8 - CONCLUSION AND RECOMMENDATIONS ............................... 172

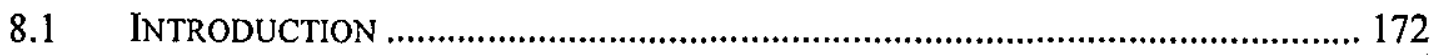

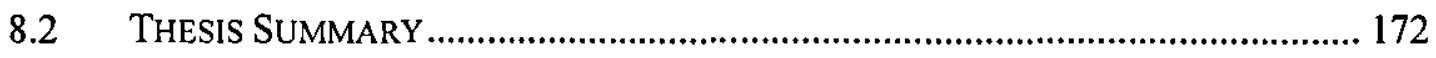

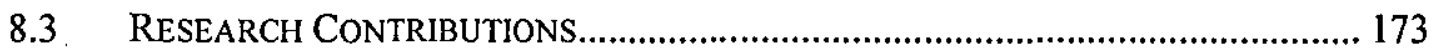

8.4 RECOMMENDATION FOR FUTURE RESEARCH ............................................. 174

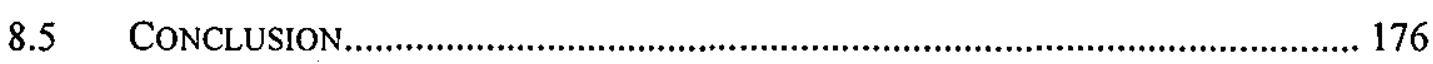

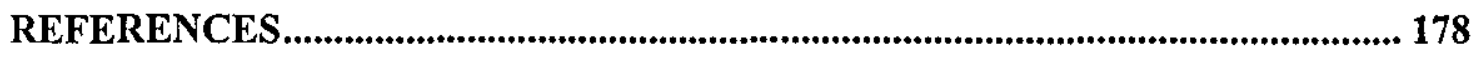

APPENDIX A - AUTHOR PUBLICATIONS............................................................ 198

APPENDIX B - SUMMARIES OF STANDARDS DOCUMENT............................... 200

APPENDIX C - TURNING FEATURES LIBRARY CODES...................................... 208

APPENDIX D - ACTIVITY DIAGRAMS.............................................................. 217

APPENDIX E - IMPLEMENTATION CASE STUDY COMPONENT 1 ............... 221

APPENDIX F - ISO 14649 PHYSICAL FILE FOR COMPONENT 1.................... 226

APPENDIX G - G\&M CODE FOR CASE STUDY COMPONENT 1.................... 231

APPENDIX H - OPERATION PLAN FOR CASE STUDY COMPONENT 2...... 234 


\section{Chapter 1 - Introduction}

\subsection{Background}

In the last few decades the economic priorities of manufacturing have shifted from being based on low cost of a standard product without compromise on consistency and quality, to the use of modern industrial manufacturing facilities with a "production on demand" concept. That concept has been adopted in order to better meet the challenges and take advantage of the opportunities of economic globalisation. This concept found expression in DABA, or "Design Anywhere, Build Anywhere" (Normile et al., 2000), where the changing business environment leads to a need to collaborate beyond geographic boundaries supported by the rapid advancement of information technology associated with manufacturing technology.

In a traditional approach design and manufacturing are considered to be separate but in a modern global and modern environment that separation becomes a major weakness leading to slow and costly production cycles. This happens because design has its own team and manufacturing has its team. The design focus is to design the product and pass information to engineers to decide how to make the product or how to realise the design. If anything happens and there is a need to redesign the product, the engineer will pass information back to the design team.

Today, with the use of computer technologies and communication technologies in the manufacturing industries, the methods mentioned above are largely being replaced by Computer Aided Design (CAD) and Computer Aided Manufacturing (CAM) to implement concurrent engineering, Widespread CADCAM systems will reduce human interaction and the result, should be increased production, reduced costs and better quality of product. 
Chapter 1

\subsection{Standard Product Data Exchange}

During the design process and production, both teams have to work closely until the final product is realised. One of the issues in that process is data transfer and data exchange. If the product is very complex such as those found in aerospace and automotive industries, the final product comes with varieties of subcomponents that have been designed by different departments using a variety tools or software. For example the part design information might be transferred using any one of a number of industry data exchange standards such as IGES, DXF and other formats. To eliminate the problem resulting from a variety of standards, new standards have been developed. ISO 10303 (informally known as STEP) and more recently ISO 14649 (informally known as STEP-NC) have been introduced as part of an international effort aimed at achieving fully interoperability and bi-directional information exchange (ISO, 2003b). To date, two different ISO subcommittees are working towards such a STEP-NC standard with two different focii; ISO TC 184/SC1 is working on ISO 14649 termed the Application Reference Model (ARM) whereas ISO TC 184/SC4 is developing STEP AP-238, termed the Application Interpreted Model (AIM). Both models represent the data model information to program intelligent CNC controllers, but the AIM is fully STEP compliant, whereas the ARM contains the information required to program a CNC machine. The ARM is to be used in an environment in which CAM systems have exact information from the shopfloor, wheres AIM is more suitable for a complete design and manufacturing integration (Xu et al., 2005).

\subsection{STEP-NC Environment for Manufacturing}

ISO 14649 is referred to as STEP-NC due to its interaction with ISO 10303 (STEP) and was initiated to provide a data model for a new breed of intelligent CNC controller that is well-structured with workplans and workingsteps. ISO 14649 aims to model the complete information requirement that must exist in a controller to control a machine tool by defining "what-to-make" and plans "how-to-make". 
STEP-NC has been developed as a result of several research projects carried out by companies and academic institutions. In terms of international research and development into these standards, projects such as OPTIMAL (ESPRIT, 1997) largely overcame the legacy standards of ISO 6983. OPTIMAL is one of the earliest STEP-compliant systems and is based on feature information and machining strategies. The research does not stop there, because the researchers now focus on identifying and defining interoperable manufacturing and STEP-NC compliance in the context of concurrent engineering. In particular, information reviews of STEP$\mathrm{NC}$, manufacturing processes and manufacturing resources are also major foci in this research area.

STEP-NC is aimed at overcoming the problems left from ISO 6983 which focuses on programming the path of the cutter centre location (CL) referred to the machine axes rather then machining tasks. One approach to the problem is to exchange a high level of information between CADCAM systems and NC controllers. STEP-NC works by manufacturing features, operations and the workingsteps. The STEP-Compliant Data programming interface for numerical controls has been introduced and proposed for standardization by the International community, where its higher level of information aims to overcome the shortcomings of contemporary NC programming. The new NC programming data model purports to support a well structured hierarchical interface, and objectoriented and two way communication from the CAD environment down to the shop floor (Muller, 2000a). STEP-NC is an improved interface between the CAD world and the manufacturing arena. It is recognized as such since it provides process information at the time and place of the manufacturing activity. The proposed STEP-NC data format supports accurate and timely adaptive control of the production equipment and provides feedback for information back to the planning activity.

The current standard of programming NC namely G \& M codes or ISO 6983 has had no significant change since the format of NC machines was developed at MIT in 1952 (Ahlquist, 2002; Allen et al., 2005; Fortin et al., 2001; Muller, 2000a; Newman, 2004b; Xu and Newman, 2006). Figure 1.1 shows the evolution of NC 
machines since using hardwired configurations to the current fully-integrated systems that can be found almost everywhere, from small job shops in rural communities to multi-national companies in large urban areas. During the preComputer-Numerical Control (CNC) epoch the program language had been modified by vendors and controller developers who added their own commands. Since the 1970's significant developments have been made towards more automatic and reliable computer numerically controlled machines with new machining processes.

Taking the Next Step in CNC Technology (Michaloski, 2005)

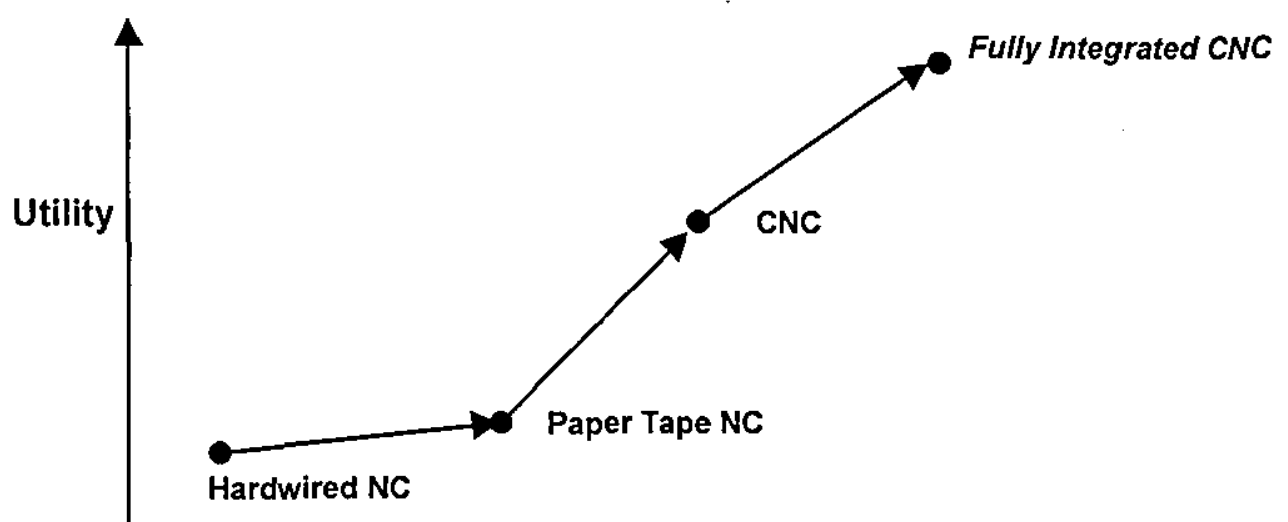

Evolution of NC Machines

Figure 1.1 : Evolution of NC Machines (Michaloski, 2005)

Today's highly sophisticated Computer Numerically Controlled (CNC) machines utilise a variety of cutting technologies such as multi-turret and multispindle in complex axial configurations and this machine capability increases the level of flexibility and capability compared to the previous decade (Nassehi et al., 2006c). A large number of Computer Aided Systems (CAx) have been developed and implemented in recent years to support all stages of product life by computer systems and many can simulate virtual CNC machining with the complete machine toolpath (Newman, 2004a). Since the first NC machine was introduced in 1952, various process plan packages have been developed and each system tried to 
interpret the part data format more reliably. Most of these systems are specialized to support certain applications, and are based on an information model that handles the application specific view of the product. These current trends are aimed at open systems but they are predominantly used in retrofitting applications for conventional NC machines. Some of the CAx systems do not share common databases for the product information due to the resistance from software and hardware vendors in terms of business strategy.

\subsection{Overall Research Intention}

Manufacturing companies invariably have more than one $\mathrm{CNC}$ machine model due to business development, technology and market demand. To date there nearly $2000 \mathrm{CNC}$ models around the globe from more than $300 \mathrm{CNC}$ machine manufacturers. Of course this variety of models needs a single standard particularly in the area of machining to improve productivity by increasing the richness of interactions and transactions. An initial standard is ISO 10303, informally known as the Standard for the Exchange of Product Model Data (ISO) which aims to provide a single International Standard for all aspects of technical products (ISO, 1994). Before that data exchange standards such as SET (Bhandarkar and Nagi, 1997), VDA and IGES (Roger et al., 1980) were proposed to meet the requirements of the CAD/CAPP/CAM industry but have still not solved the portability and interoperability issues.

The majority of researchers have focused their attention on milling operations, while this research opens the opportunity for the author to explore the lack of knowledge in the area of turning. During this research, commercial software from established $\mathrm{CNC}$ controllers has still not been launched as the products are still at the research and development stage. In terms of ISO documents, not all ISO 14649 documents have been finalised, some are at the draft version and still subject to discussion among ISO committee members (ISO TC184 SC1 and SC4). 
This thesis presents a comprehensive literature review of the background to the evolution of $\mathrm{CNC}$ manufacturing, past and current standards for machine programming and the current implementation of a new standard focusing on ISO 14649 or STEP-NC. The relevant research issues for the development and introduction of reconfigurable machines tools are presented focussed on turning operations. The section of this thesis focused on theoretical research consists of the proposed system framework and information models. For the experimental research section, a prototype STEP-NC compliant CAD/CAPP/CAM system has been implemented and an industrially-oriented case study has been developed to illustrate and demonstrate this system. Finally, the conclusions express the realisation that many opportunities exist beyond the boundaries of this research for future work related to global interoperable STEP-NC systems and a full solution is a long way away even with full commitment from all participants.

\subsection{Overview of Research Problem}

Much software and hardware has been developed around the globe to organise and integrate the different phases of a product life-cycle management (PLM) in conception, design, manufacture, service or disposal phases. The research in this thesis focuses just on the design (CAD) and manufacture (CAPP, CAM and $\mathrm{CNC}$ ) where there is potential to integrate a wide variety of software applications. In order to achieve this principal aim, a number of research problems have been identified.

1.5.1 The current $\mathrm{CNC}$ regime is considered to rely on low level codes such as the description of tool movements and switching instructions. Today with the latest technology the information beyond tool movement and switching instruction such as tooling, manufacturing features and process sequences are needed to support global adaptability for manufacturing with a specific focus on CNC-based manufacture. STEP-NC is considered to have the 
necessary rich information including "what to make" and "how to make".

1.5.2 Interoperability problem; particular companies use various systems for different components which come from more than one supplier and need to be able to exchange information reliably and rapidly. Another example is when a manufacturer needs to move machining operations to another location. The new location has a different software applications for the same function, so all software programs must be rewritten, potentially resulting in very considerable cost because of product launch delay, additional labour costs and loss of product quality.

1.5.3 ISO 6983, commonly known as $\mathrm{G}$ and $\mathrm{M}$ codes, focuses on programming the path of the cutter centre location (CL) with respect to the machine axes, rather then the machining task with respect to a part.

\section{$1.6 \quad$ Aims}

This research aims to explore the application of new data standards for CNC machining of turned parts to enable data exchange of "manufacturing rich" information from CAD to $\mathrm{CNC}$ to support interoperable manufacture.

\subsection{Objectives}

The main area of interest is to identify and define interoperable manufacturing and STEP-NC compliance in the context of concurrent engineering. In particular the information review of Standards for the Exchange of Product Model Data for Numerical Controls (ISO, 2005a), manufacturing processes, and manufacturing resources provides the basis for this research. In order to achieve this goal, the following objectives have to be accomplished:

1.7.1 Review the literature relating to relevant STEP standards and application in the area of turning centres. This research will review the various research 
work, carried out from the evolution of STEP-NC of the CNC manufacturing activities.

1.7.2 Design of a prototype interoperable STEP-NC compliant system for turning operations. One important aspect in this research is the design of a STEP compliant CAD/CAPP/CAM environment which conforms to the proposed framework.

1.7.3 Creation of a STEP-NC compliant information model for turning centre component manufacturing. This research aims to define two fundamental information models, namely the product and manufacturing models, representing the vital information required in the proposed system.

1.7.4 Creation of a computational environment for the STEP-NC compliant system for turning operations. The experimental part of the research will involve the development of a STEP-NC compliant system for turning operations to demonstrate the validity and effectiveness of the data model.

1.7.5 Case study implementation for testing the prototype system. The STEP-NC compliant system for turning operations will be evaluated with case study components, based on an example in ISO14649-12 standard and other industrial components.

\subsection{Organisation of the thesis}

This thesis focuses on the use of the new standard; ISO 14649 (STEP-NC), to address the process planning and machining of turn/mill discrete components. It explores how ISO 14649 can be used to combine turning and milling operations to support interoperable $\mathrm{CNC}$ manufacturing of rotational components at a single turning centre. This thesis is presented in eight chapters. 
Chapter 1: Presents a brief introduction of the topic to be investigated, identifying the motivations which have led to this research. The aims of the research and its objectives are outlined with a clear identification of the novel content of the research.

Chapters 2: The first part of literature review, focuses on ISO and the standards, and reviews standards for information exchange including the current status of ISO 14649 documents.

Chapter 3: The second part of the literature review, focuses on STEP-Compliant process planning and manufacturing, implementation in machining research work related to STEP-NC is discussed.

Chapter 4: Specification of a STEP Compliant system for Turning Operations. The design and specification has been developed on the basis of a detailed literature review and current systems developed by other developers and researchers.

Chapter 5: Information model - This chapter discusses efforts underway to make STEP information models available in universal formats familiar to most business application developers: specifically XML and the Unified Modelling Language (UML).

Chapter 6: Outlines the development of a prototype system based on the system framework and information model identified in the previous chapter, including design stage, implementation stage and the use of the SCSTO system. The computational environment for the prototype system is described in this chapter in terms of the development and functional and operational structure to generate a Part 21 file for turning operations. The SCSTO system is based on the data model described in ISO 14649 Part 10, 12 and 121 documents. The 
information content is composed of i) task description, ii) technology description, iii) tool description and iv) geometry description.

Chapter 7: Describes the case study components evaluated with the prototype system. It involved two components based on an ISO 14649 Part 12 example and an industrial part to show the complexity levels from standard turning machines to multi axis machines.

Chapter 8: Summarises the achievements based on the objectives outlined and describes the anticipated impact. The novel and unique engineering and scientific contributions which have been delivered through this research are presented. The future work that might be carried out is proposed, based on the experiences and lessons learned in this research by identifying directions for future research in this area.

In addition, Appendix A provides related published papers by the author on various aspects of the research. 


\section{Chapter 2 - Product Data Model and Related Standards}

\subsection{Introduction}

This literature survey aims to give an overview of the standards that are used in manufacturing process applications and computer aided software (CAx) systems to support the integration of design and manufacturing activities. Since the birth of the first NC milling machine at MIT, a huge number of process plans for machine parts have been developed all over the world (Aslan et al., 1999). CAx systems are developed separately with different data formats, so there is a need to exchange product data between systems. Product data exchange standards have been introduced, such as IGES, SET, STEP, etc. to facilitate the data exchange between different systems and attempted to interpret part data into various formats (Goldstein et al., 1998). In this research, standard documents refer to the field of industrial automation and integration concerning discrete part manufacturing. The first part of this chapter describes the conventional methods of part programming, and the data transfer standard representing and exchanging the product data model. The final part of the chapter focuses on STEP-NC compliant machining process planning.

\subsection{Product Design Representation}

There are two main approaches in terms of product design representation; i) geometric modelling and ii) feature modelling. Gu and Norrie have classified 
geometric modelling into two groups; $2 \mathrm{D}$ and 3D, based on wire frame, surface or solid models ( $\mathrm{Gu}$ and Norrie, 1995). In this thesis the focus is on feature modelling and brief summaries are presented of feature based CAD/CAM and feature research related to rotational parts.

\subsubsection{Feature-Based CAD/CAM}

Over the past last two decades significant research effort has been focused on feature based part modelling, process planning and $\mathrm{CAD} / \mathrm{CAM}$ integration in order to make it product design and manufacturing fully automated and integrated. One very popular topic for research since the 1980s is feature recognition and many methods have been proposed including syntactic pattern recognition, rule-based search, graph-based matching, volumetric decomposition, hint-based geometric reasoning, neutral networks and generic algorithm-based methods (Zhou et al., 2007). In terms of feature-based design or design by features, Dong et al., have defined as "feature-based design provides designers with a feature library in which a number of features are predefined and when designers design a part, they can select different features to form the parts" (Dong et al., 1996). Huang et al. suggested that a feature is a volume whose shape is an instance of some member of a predefined set of shape types (Huang et al., 2001). Case and Harun have stressed that features are not only for a particular application but also need to be used as the crucial integrating aspect of product models (Case and Harun, 2000). In terms of ISO standards, ISO 10303-224 does not define explicitly a feature but, in section 4 of this Application Protocol the Unit of Functionality (UoF) manufacturing feature (manufacturing_feature) is described as follows "...contains the information necessary to identify shapes which represent volumes of material that shall be removed from a part by machining" (ISO, 2000b). ISO 10303-224, named AP224, is concerned with machining features and that is reflected in the definition.

Fidan presented STEP AP224 features based modelling for rotational parts and his thesis covers features extracted from STEP AP224 for rotational parts and also gave the definitions, classifications, attributes, generation techniques, attachment methods and geometrical constraints (Fidan, 2004). Wang and Zhang 
have classified a model consisting of tree sub-models including product information model, component information model and part feature information model as shown in figure 2.1 (Wang and Zhang, 2002).

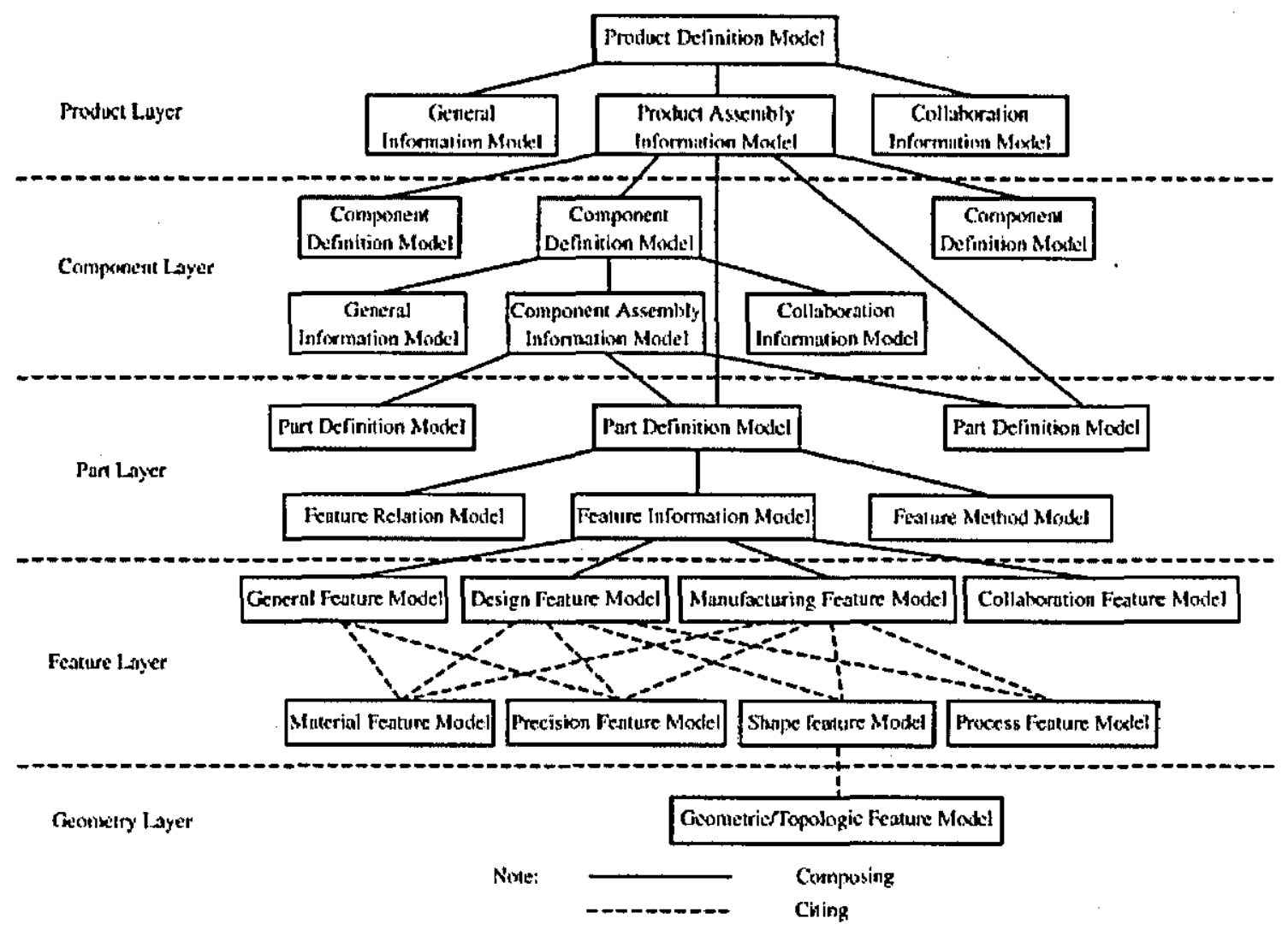

Figure 2.1 General structure of product definition model (Wang and Zhang, 2002)

A list of more than 100 CAPP systems is provided by Alting and Zhang, and these systems are divided into; i) variant CAPP systems (based on Group Technology coding), ii) generative CAPP systems (based on built-in decision making logic), iii) hybrid CAPP systems (which combine variant and generative approaches), and iv) knowledge-based CAPP systems (based on expert systems) (Alting and Zhang, 1989). Other systems have used feature-based approaches such as the prototype developed by $\mathrm{Lin}$ et al. that was integrated with a commercial CAD/CAM package for extracting machining features from the CAD model and called CAFÉ (Lin et al., 1997). Lee and Kim presented an approach for incrementally extracting machining features from a feature-based design model as a 
design evolves and implemented it as part of a feature-based modelling system (Lee and Kim, 1998). Patil et al. developed a feature-based process planning system that consists of a feature-based modeller and an automatic process planner to generate NC code for prismatic parts (Patil and Pande, 2002). Most recently, Amaitik et al. have developed an intelligent process planner using STEP features called STEP-FM which is also for prismatic parts (Amaitik and Kilic, 2007).

\subsubsection{Feature Research Related to Rotational Parts}

Li successfully developed a system for a part feature recognition for a conversion language from CAD to CAM for rotational parts ( $\mathrm{Li}, 1988)$. Yang el at. introduced a case-based process planning system called PROCASE which generates new process routines through learning from existing process routines for machining of rotational parts (Yang et al., 1994). Tseng and Joshi extended the feature recognition domain to include the classes of parts with interacting rotational and prismatic features to develope the machining volume generation method (Tseng and Joshi, 1998). Subsequently Aslan et al. proposed the approach of starting from part design, extracting data from the CAD model and finally making preparations for a feature called FMPA for rotational parts (Aslan et al., 1999)

Shunmugam et al. (2002) described a method for preliminary process planning to determine feature sequence and precedence in asymmetric parts using genetic algorithms. They categorised the features in a hierarchical structure that has primary features (face, cylinder and cone) and secondary features (groove, chamfer, thread and $\mathrm{C}$-axis features). In their structure the $\mathrm{C}$-axis features are a radial hole, axial hole, slot and keyway. Figure 2.2 depicts the hierarchical structure for the form features. Fidan developed a STEP AP224 feature generator where the features were used as the basic entities for part design consisting of three phases i) feature library, ii) feature modeller and iii) pre-processor (Fidan, 2004). Yildiz et al. developed an automatic feature recognition and $\mathrm{CNC}$ code generation system for rotational parts using the Delphi 7 programming language and using DXF format as data input to the system (Yildiz et al., 2006). 


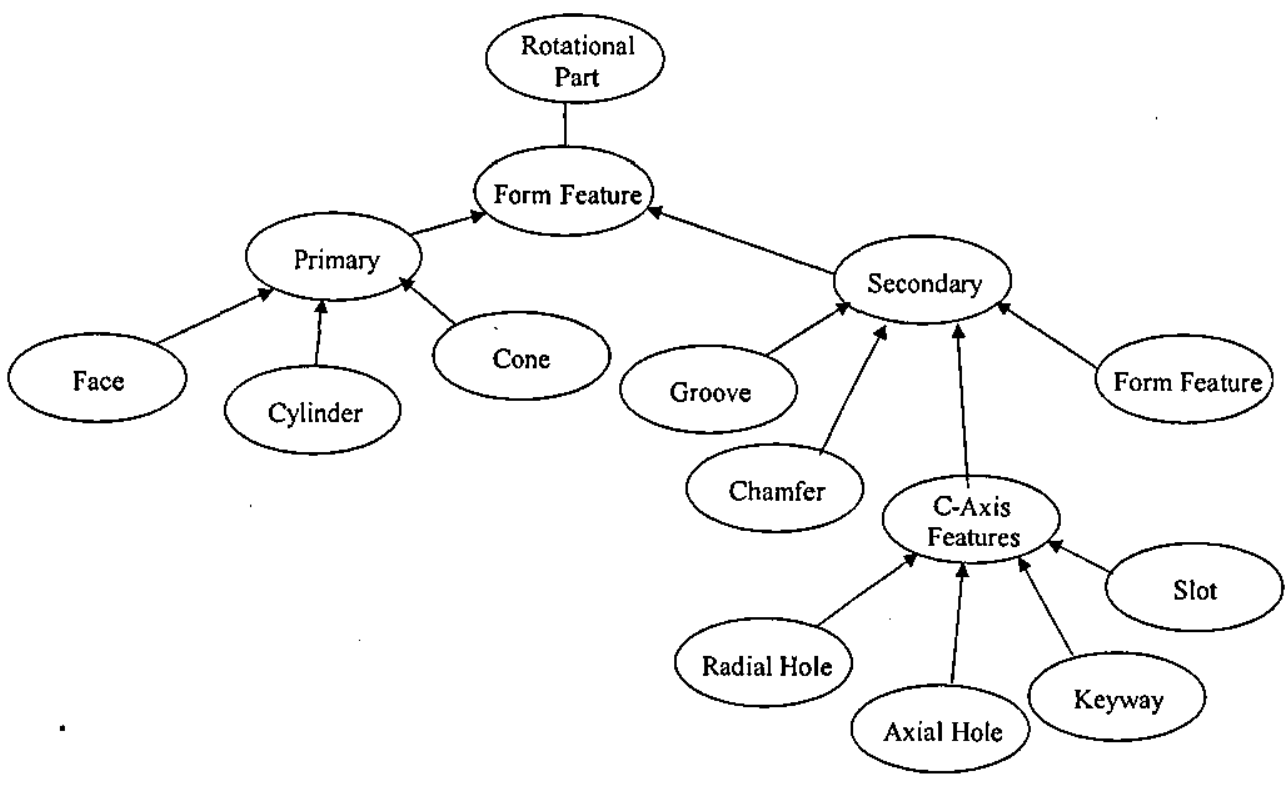

Figure 2.2 The hierarchical structure of form features proposed for asymmetric parts (Shunmugam et al., 2002)

\subsection{Product Data Model}

The three main reasons for transferring data among CAD systems (Vergeest, 1991) are, i) to communicate with remote co-workers or colleagues in the company, ii) long duration storage and retrieval for archival purposes, and finally iii) during the design process. This section describes selected data transfer standards relying on the neutral data format that have been developed in the past such as the Drawing Transfer File (DXF) and the Initial Graphics Exchange Standard (IGES) (Xu and $\mathrm{He}, 2004)$. Kemmerer outlines the evolution of product definition capabilities, as shown in figure 2.3 (Kemmerer, 1999). This shows that the first product definition was in the form of physical models. From the 1800s up to the late 1900s engineering was the major form of product data transfer, with today's product digital drawings being represented in the form of intelligent product data (Kemmerer, 1999). Ever since the beginning of CAD and CAM software, the problem of a model's portability from system to system was one of the key issues affecting the spread in the use of these tools. Many solutions were proposed for data exchange in past such as SET (Standard d'Echange et de Transfert, 1985), VDA 
(Verband Der Automobilindustrie Flachen Scnittstelle, 1986), and IGES (Roger et al., 1980), which were partially successful but were not totally suitable for all the needs of the CAD/CAPP/CAM industry (Kemmerer, 1999).

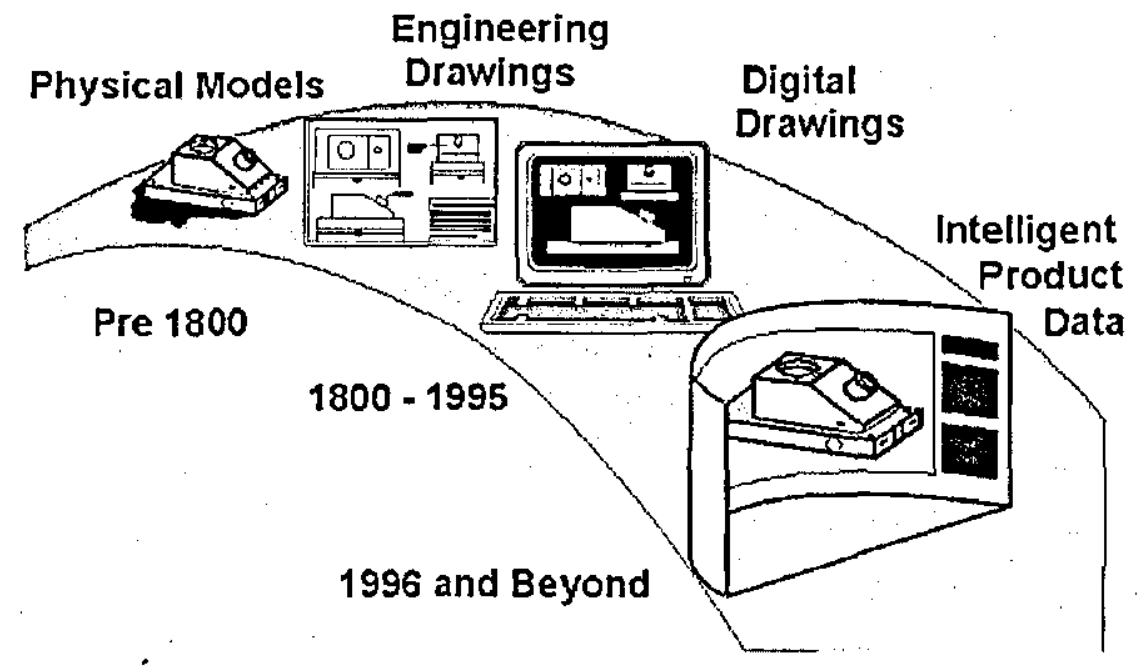

Figure 2.3 Evolution of Product Definition Capabilities (Kemmerer, 1999)

Figure 2.4 based on information from the Automotive Industry Action Group (AIAG, 1997), identifies some of the different CAx platforms used by members of the U.S. automobile supply chain between first-tier, sub-tier and tooling suppliers.

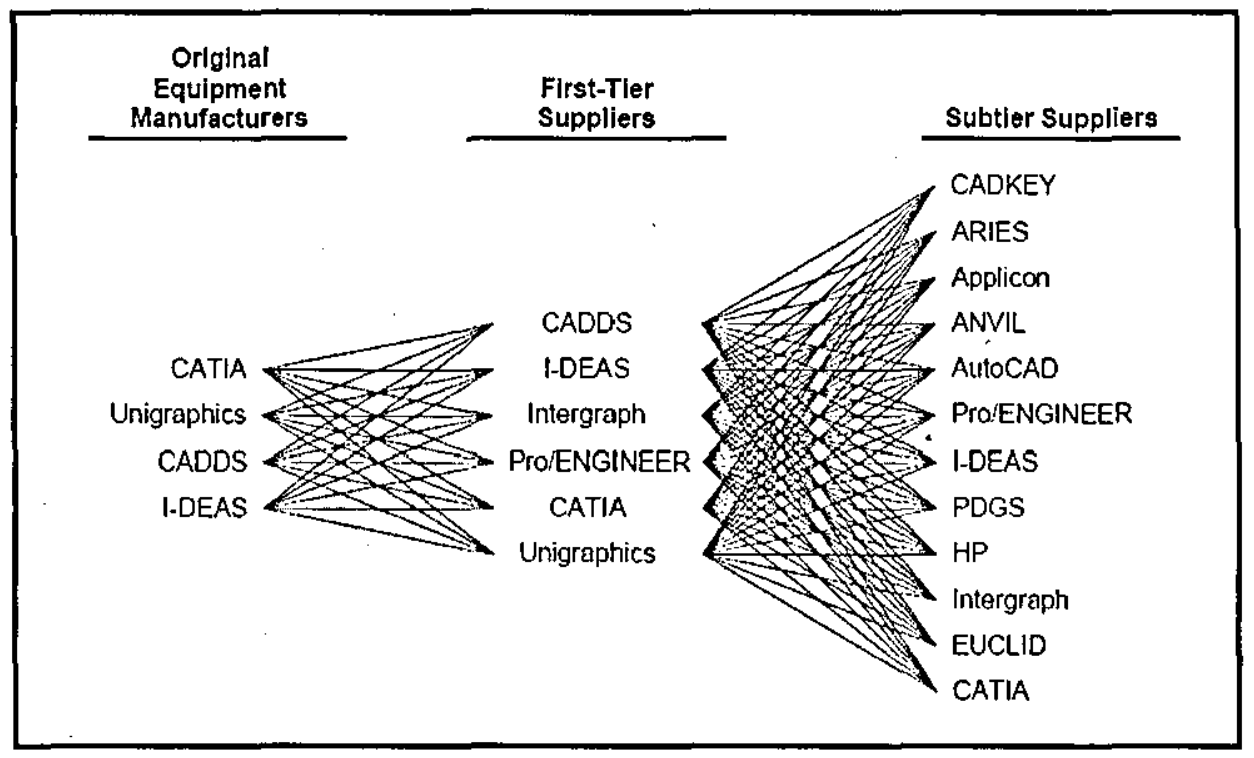

Figure 2.4 Multiples CAx systems in US automobile supply chain (AIAG, 1997). 


\subsubsection{Drawing Transfer File (DXF)}

The Drawing Interchange file format (DXF) was developed as an industrial standard to assist in interchanging drawings between AutoCAD and other programs. The AutoCAD drawing database (.dwg file) is written in a compact format that changes significantly as new features are added to AutoCAD. All implementations of AutoCAD accept this format and are able to convert it to and from their internal drawing file representation. DXF files are standard ASCII text files, so can easily be translated to the formats of other CAD systems or submitted to other programs. Fidan (2004) reported that at early versions of AutoCAD, more attention was paid to drafting rather then being focused on solid $3 \mathrm{D}$ models as used by most other CAD packages for building and construction, aerospace, automotive, shipbuilding, electrical, industrial, and consumer products (Fidan, 2004). When addressing CAD data exchange, DXF does not include the scope of product model data that is included in STEP (discussed in 2.4).

\subsubsection{Initial Graphics Exchange Standard (IGES)}

In 1979, a series of events catalysed the CAD vendor and user community to create the first national standard for CAD data exchange, which is documented in the Initial Graphics Exchange Specification, version 1.0 (Roger et al., 1980). The first draft of IGES was released in 1980, containing geometry, graphical data and annotations (Goldstein et al., 1998). The name was carefully chosen to avoid any suggestion of a database standard that would compete with the proprietary databases then used by the different CAD vendors. In early versions, IGES defined a collection of information structures, either of geometry or non-geometry format using ASCII codes. The geometry entities consisted of simple physical shapes, solid model and part features, while the non-geometry entities consisted of graphical characteristics such as dimension and text (Bhandarkar et al., 2000). Today, IGES is still used as a universal tool, providing a neutral format for many companies to transfer engineering data between $\mathrm{CAD} / \mathrm{CAM}$ systems, but usually in terms of $2 \mathrm{D}$ drawing and possibly wireframe models (Ravat and Nazemetz, 2007). Subsequently 
the IGES community made some revisions. The main problem in the context of geometry processing is that the IGES file does not contain information about the topological structure of the surface components. Therefore, it was necessary to convert the IGES description into an internal model that contained structural and metric information.

\subsubsection{PDDI and PDES}

The Product Definition Data Interface (PDDI) was developed by McDonnell Aircraft Corporation from the ICAM (Integrated CAM) project (Shah and Rogers, 1988). The standard format was intended to serve as the information interface between engineering and all manufacturing functions including process planning, numerical control programming, quality assurance, tool design and others (Goldstein et al., 1998; Zeid, 1991). The PDDI was interfaced with two commercial CAD systems to demonstrate its general applicability. Although the PDDI standard format could cater for discrete mechanical components such as sheet metal, turned, composite and machined configurations, it could not deal with component assemblies.

The Product Data Exchange Specification (PDES) is a United States organisation activity initialised by the IGES organisation aiming to establish a mechanism for complete product model data exchange (Shah, 1998). The PDES initiation effort began in 1986 and was a "proof of concept" project begun within the IGES organization to validate the methodology by which PDES would develop into a product data exchange specification (Gallaher and O'Connor, 2002). The documents developed as part of the PDES activity are periodically submitted to the International Standards Organization (ISO) in support of the STEP effort.

The ISO Technical Committee 184/Sub-Committee 4 (TC184/SC4) has driven the effort to replace the bewildering array of emerging data exchange standards and agree an international standard for representing and exchanging the product data. Finally the TC184/SC4 has coordinated and drawn together several world wide projects including the VDA-FS, (Verband Der Automobilindustrie 
Flachen Scnittstelle, 1986), standard file format to exchange surface data which is popular in Europe due to the endorsement by a group of 27 major stakeholders, and European car manufacturers such as DaimlerChrysler, Audi, BMW and Volkswagen. SET (Standard d'Echange et de Transfert, 1985), PDDI and PDES were combined into a single unified global standard specified as ISO 10303 (ISO, 1994) or more commonly known as STEP.

\subsection{The Standard ISO 6983}

The manual part programming in this research is defined as the use of $G$ \& $M$ codes based on ISO 6983; data format for positioning, line motion and contouring control systems (ISO, 1982). Two main formats have been defined in G \& $M$ codes, namely preparatory functions ( $G$ codes) such as $G 00$ for positioning (rapid transverse), G01 for linear interpolation, G02/G03 for circular interpolation and G99 return to reference point. The second set of functions known as miscellaneous functions ( $\mathrm{M}$ codes) include M00 for program stop, M03/M04 for spindle rotation either clockwise or counter clockwise, M06 for tool change and M99 for end sub program. This conventional method is considered as providing low level information due to the ability to provide just the centre line of the cutting tool path and simple switching instructions and as it only supports one way information flow from design to manufacturing (Xu and Newman, 2006; Xu et al., 2005). Childs (1973) has pointed out that there are only five instructions in NC part programming which specify;

i. Which tool to use (tool selection)

ii. How fast the tool turns (spindle speed)

iii. How fast the tool moves (feed rate)

iv. How the tool moves (machining mode) and

v. Where the tool moves to (coordinates) 
The preparatory and switching codes are address words for the controller as shown in table 2.1 and a typical example line of ISO 6983 part programming code is shown in figure 2.5 .

Table 2.2 The codes are address words; adapted from (Srinivas, 2004).

\begin{tabular}{|l|l|l|}
\hline No & Word address format & Purpose \\
\hline 1 & N & Operation sequence \\
\hline 2 & G & Preparatory function \\
\hline 3 & X,Y,Z & Dimension address \\
\hline 4 & S & Spindle \\
\hline 5 & F & Feed rate \\
\hline 6 & T & Tool address \\
\hline 7 & M & Miscellaneous functions \\
\hline
\end{tabular}

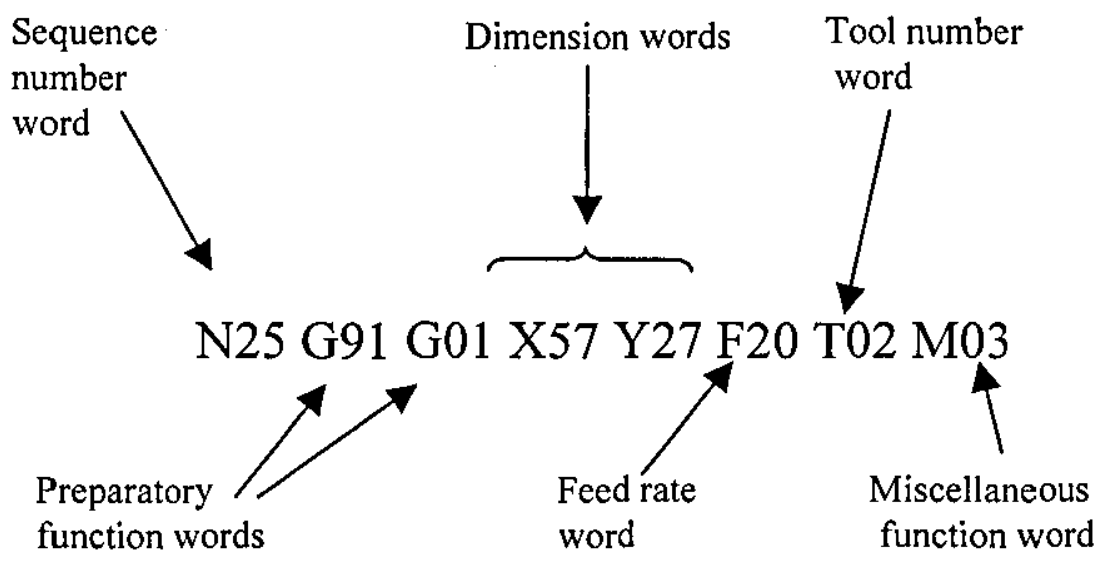

Figure 2.5 Program line in ISO 6983 code, adapted from (Williams, 1988)

$\mathrm{Xu}$ and $\mathrm{He}$ (2004) stated that a number of problems are found with ISO6983 including;

i. The language focuses on programming the path of the cutter centre location (CL) with respect to the machine axes, rather than the machining tasks with respect to the part. 
ii. Vendors usually supplement the language with extensions that are not covered in the limited scope of ISO 6983 and hence the CNC programs are not exchangeable.

iii. The CAD data are not used directly on the machine, instead, they have to be processed by a machine-specific post-processor, only to obtain a set of low-level, incomplete data that makes verification and simulation difficult, if not impossible.

Mechanical parts are becoming more and more complicated, increasing the preparation work and set-up time on CNC machine tools. In terms of an economic perspective, Kovacic and Balic (2003) report that the cost of programming CNC machine tools accounts for about $30 \%$ of the production costs of single parts or small batches, therefore rational programming of $\mathrm{CNC}$ machine tools is very important .

\subsection{Standard for the Exchange of Product Model Data (STEP)}

The standard, ISO 10303 is informally known as the Standard for the Exchange of Product model data, (STEP) and is much broader than IGES, covering the entire life-cycle of a product. STEP is an unofficial name; the actual designation of the STEP standard is ISO 10303 Industrial automation systems - Product data representation and exchange (ISO, 1994). STEP provides a computer interpretable representation and exchange of product data. Product data represents information about a product in a formal manner suitable for communication, interpretation, or processing by human beings or by computers. The objective of STEP is to provide a neutral mechanism capable of describing product data throughout the life cycle of a product independent from any particular system. The nature of this description makes it suitable not only for neutral file exchange, but also as a basis for implementing and sharing product databases and archiving (Peng and Trappey, 1998). 
In a modern global manufacturing environment, data exchange is commonly understood to be the exchange of neutral format data files between computer systems either based on data exchange; data translation and data sharing as shown in Figure 2.6 (Amaitik, 2005).

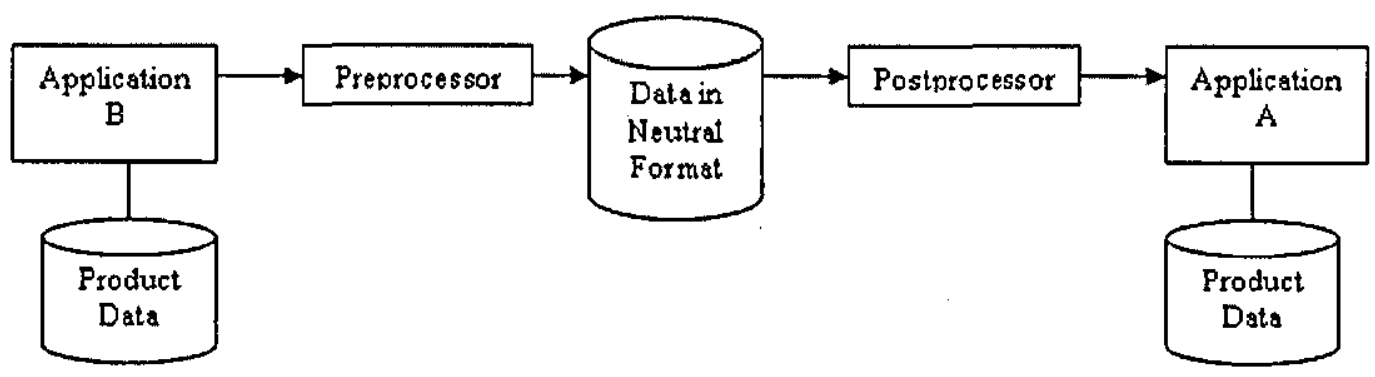

a) Data translation

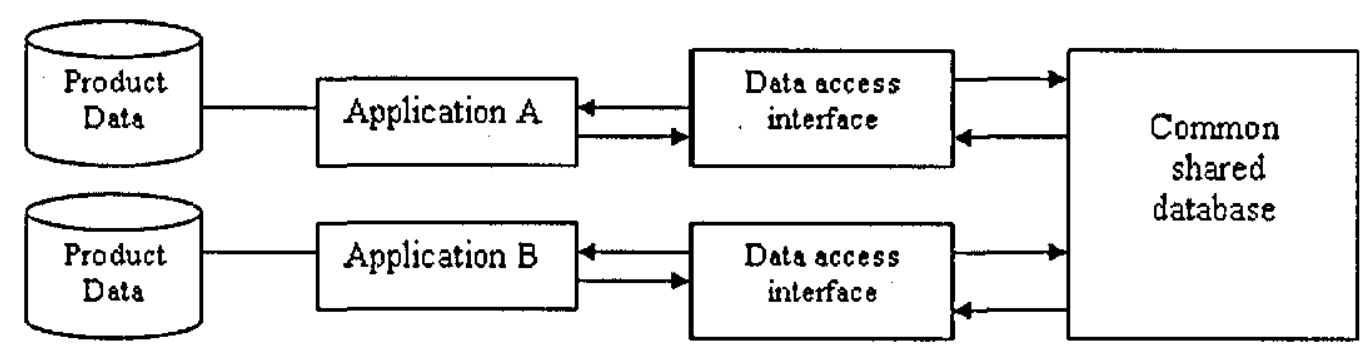

b) Data sharing

Figure 2.6 Product data exchange mechanisms (Amaitik, 2005)

\subsubsection{The Components of STEP}

STEP, or ISO 10303, covers a wide variety of different product types such electronic, mechanical, architectural and others, and life cycle stages such as design, planning and manufacture. The standard was designed to support the needs of many different industries and disciplines to exchange product data. The component of the STEP standard that addresses the specific needs for product data exchange of a particular industry sector, engineering discipline or CAD/CAM application technology area is called an Application Protocol (AP). Each Application Protocol 
defines the basis for implementing interfaces for the exchange of product data between similar applications from different software vendors. Further, the AP defines a basis for assessing these interfaces for conformance to the requirements of the standard. STEP is published as a series of Parts (e.g., Part 11 is ISO 1030311:1994). There are four major components of an Application Protocol:

i. Scope and activity model information about what the Application Protocol covers and what it excludes.

ii. Information requirements to describe the types of data to be exchanged.

iii. Standardized data specification to govern the data exchange (i.e., data structures, relationships, semantics and constraints) which contains the fully specified EXPRESS data model to be used.

iv. Conformance requirements to define the implemental subsets of the AP for exchange purposes.

\subsubsection{STEP Data Model Structure}

The STEP data model structure consists of information models that are shown shaded and infrastructure shown not shaded in figure 2.7 and uses the formal specification language called EXPRESS (Rahimifard and Newman, 1996). Figure 2.7 also shows that STEP is organised as series of parts, each published separately and consisting of description methods, integrated resources, application protocols, abstract test series, implementation methods and conformance testing (Kemmerer, 1999). Description methods are standardised in the ISO 10303-10 series of parts. Application protocols (APs) are the implemental data specifications of STEP. APs include an EXPRESS information model that satisfies the specific product data needs of a given application context.

An abstract test suite contains the set of abstract test cases necessary for conformance testing of an implementation of a STEP application protocol. Each abstract test case specifies input data to be provided to the implementation under test, along with information on how to assess the capabilities of the implementation. Implementation methods are standard implementation techniques for the 
information structures specified by the STEP data specifications intended for implementation, application protocols. Each STEP implementation method defines the way in which the data constructs specified using STEP description methods are mapped to that implementation method. The conformance testing methodology and framework series of 10303 parts provide an explicit framework for conformance and other types of testing as an integral part of the standard. This methodology describes how testing of implementations of various STEP parts are accomplished (Kemmerer, 1999).

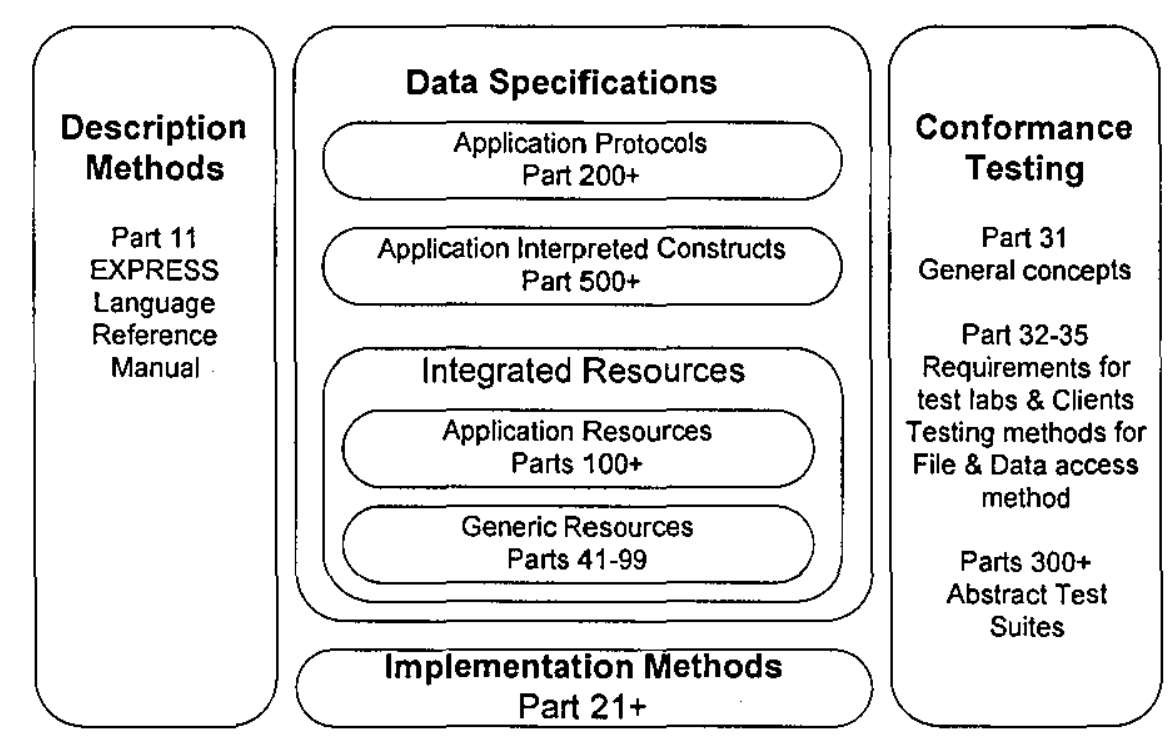

Figure 2.7 STEP architecture (Kemmerer, 1999).

\subsubsection{Express Language}

ISO 10303 Part 11 is a formal data specification language referred to as EXPRESS and is used to specify the representation of product information (ISO, 2004c). The EXPRESS family of languages has been, and is being, developed under the auspices of ISO TC184/SC4. EXPRESS itself is an object-flavoured lexical language for information modelling (Wilson, 1998). The EXPRESS Language Reference Manual also defines a graphical subset of the lexical language called EXPRESS-G. The third member of the family is called EXPRESS-I and is a lexical language for the display of data instances and also for the formal definition of test cases (Rahimifard and Newman, 1996). Schenck and Wilson provide a more user- 
oriented view of these languages than the ISO standard documents, and also outlines a modelling methodology (Schenck and Wilson, 1994). EXPRESS defines information models as schemas and each schema contains entities ancillary types, functions and rule definitions. The use of a formal language enables consistent representation of information within the data model and provides one definition of the manufacturing data common to many applications (Rahimifard and Newman, 1999).

\subsection{STEP-NC Compliant Machining Process Planning}

STEP-NC was initiated in the late 1990s to provide a data model for a new breed of intelligent $\mathrm{CNC}$ controller. Since its initial specification it has continuously evolved through third parties namely; vendors, users and academics from across the world. Major academics and industrialists involved in this standard are Aachen and Stuttgart Universities, Siemens in Germany, Pohang University, and ERC-ACI in Korea, CADCAMation in Switzerland and NIST with STEP Tools in the USA (Newman, 2004b). STEP-NC was introduced to an ISO Working Group in 1997 as a Draft International Standard (DIS) called ISO 14649 by ISO Technical Committee TC184/ subcommittee SC1 (Xu and He, 2004). ISO 14649 Part 1 (ISO, 2003b) provides an overview of the fundamental principles of the standard.

\subsubsection{ISO 14649 Purpose}

Since ISO 14649 aims to replace the legacy standard of NC programming, ISO 6983 and others, the purpose of ISO 14649 is (ISO, 2003b):

i. To cover the current and expected future needs for data exchange

ii. To support the direct use of computer-generated product data from ISO 10303

iii. To create an exchangeable, workpiece-oriented data model for CNC machine tools

iv. To be used as a standard for modern languages and libraries for the 
implementation of the data model;

v. To ensure compatibility of CNC input data.

ISO 14649 is applicable to advanced CNC machine tool and CAM systems. Figure 2.8 shows the manufacturing life cycle, from design to fabrication, and how ISO 14649 is envisioned to be used within this cycle. The design phase results in CAD data (ISO 10303-203 geometry) and includes the definition of all the part features in ISO 10303-224 (ISO, 2000b). The process planning phase generates the resource requirements for part fabrication, using ISO 10303-213, and other results suitable for use in a Manufacturing Execution System (MES). Process planning also splits the ISO 10303-224 manufacturing features into sets suitable for various processes, e.g. milling, turning, electrical discharge machining (EDM), and inspection (which also uses ISO 10303-219).

STEP AP238 Integrated resources, vla SDAl

STEP ISO 10303-224

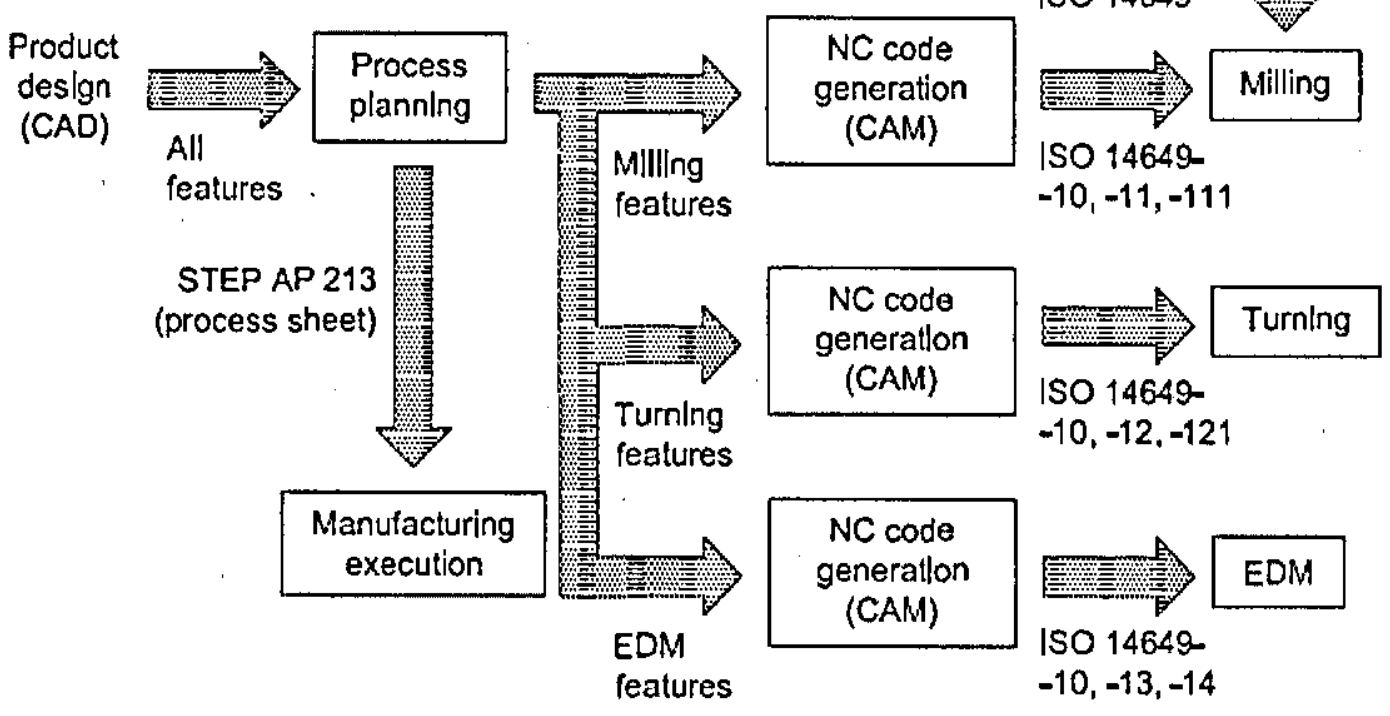

Figure 2.8 The manufacturing cycle, from design to fabrication, and how ISO 14649 is envisioned to be used within this cycle (ISO, 2003b) 


\subsubsection{ISO 14649 Parts}

ISO 14649 has several parts, covering machining data, process specific data and tool requirements for; milling, turning, wire and die-sink electrical discharge machining (EDM) and inspection (Proctor et al., 2002). A summary of ISO10303 and ISO 14649 related to this research can be found in Appendix B and each Part can be summarised as;

i. Part 1: Overview and fundamental principles - includes a data model for computerized numerical controllers, an overview and fundamental principles (ISO, 2003b).

ii. Part 10: General Process Data - specifies the process data that is generally needed for NC programming within all machining technologies and describes the interface between a computerized numerical controller and the programming system (ISO, 2004a).

iii. Part 11: Process Data for Milling - specifies the data elements needed as process data for milling. Together with the general process data described in ISO 14649-10 this part of ISO14649 describes the interface between a computerized numerical controller and the programming system (ISO, 2004b).

iv. Part 12: Process Data for Turning - specifies the data elements needed as process data for turning (ISO, 2005a).

v. Part 13: Process Data for wire-EDM - specifies the data elements needed as process data for wire-EDM.

vi. Part 14: Process Data for sink-EDM - specifies the data elements needed as process data for wire-EDM.

vii. Part 16: Data for touch probing based inspection - specifies the data elements needed as process data for touch probing based inspection.

viii. Part 111: Tools for Milling Machines - specifies the data elements needed as tools for milling. These data elements can be used as the criteria for selecting one of several milling and drilling type tools, not to describe the complete information about a particular tool (ISO/FDIS, 2004). 
ix. Part 121: Tools for Turning Machines - specifies the data elements needed as tools for turning. The turning_tool_schema defined in this part of ISO 14649 serves as a basic tool schema that includes only the most important information (ISO, 2005b).

Figure 2.9 illustrates the activities and related standards involved in manufacturing operations. It should be noted that STEP-NC lies under machining and on-line inspection and has been developed for use at CNC workstations. This STEP-Compliant Data programming interface for numerical controls has been introduced and proposed for standardization by the International community, where its higher level of information aims to overcome the shortcomings of contemporary NC programming. The new NC programming data model purports to support a well structured hierarchical interface, and object-oriented and two way communication from the CAD environment down to the shop floor (Muller, 2000c; Muller, 2003). STEP-NC is an improved interface between the CAD world and the manufacturing arena. It is recognised as such since it provides process information at the time and place of the manufacturing activity. The proposed STEP-NC data format supports accurate and timely adaptive control of the production equipment and provides feedback for information back to the planning activity. The main advantages of the new STEP-NC data interface presented in the report by Muller are as follows (Muller, 2004):

i. The STEP-NC data interface will be a neutral data description: CAM independent, NC machine-tool independent. This would significantly enhance exchangeability and flexibility, and allow considerable cost reduction as machine or controller specific data will no longer be needed.

ii. Straightforward information exchange using CAD standard STEP. Since geometry of parts is described using STEP syntax, a direct exchange of information between CAD, CAM and NC can be realized. 


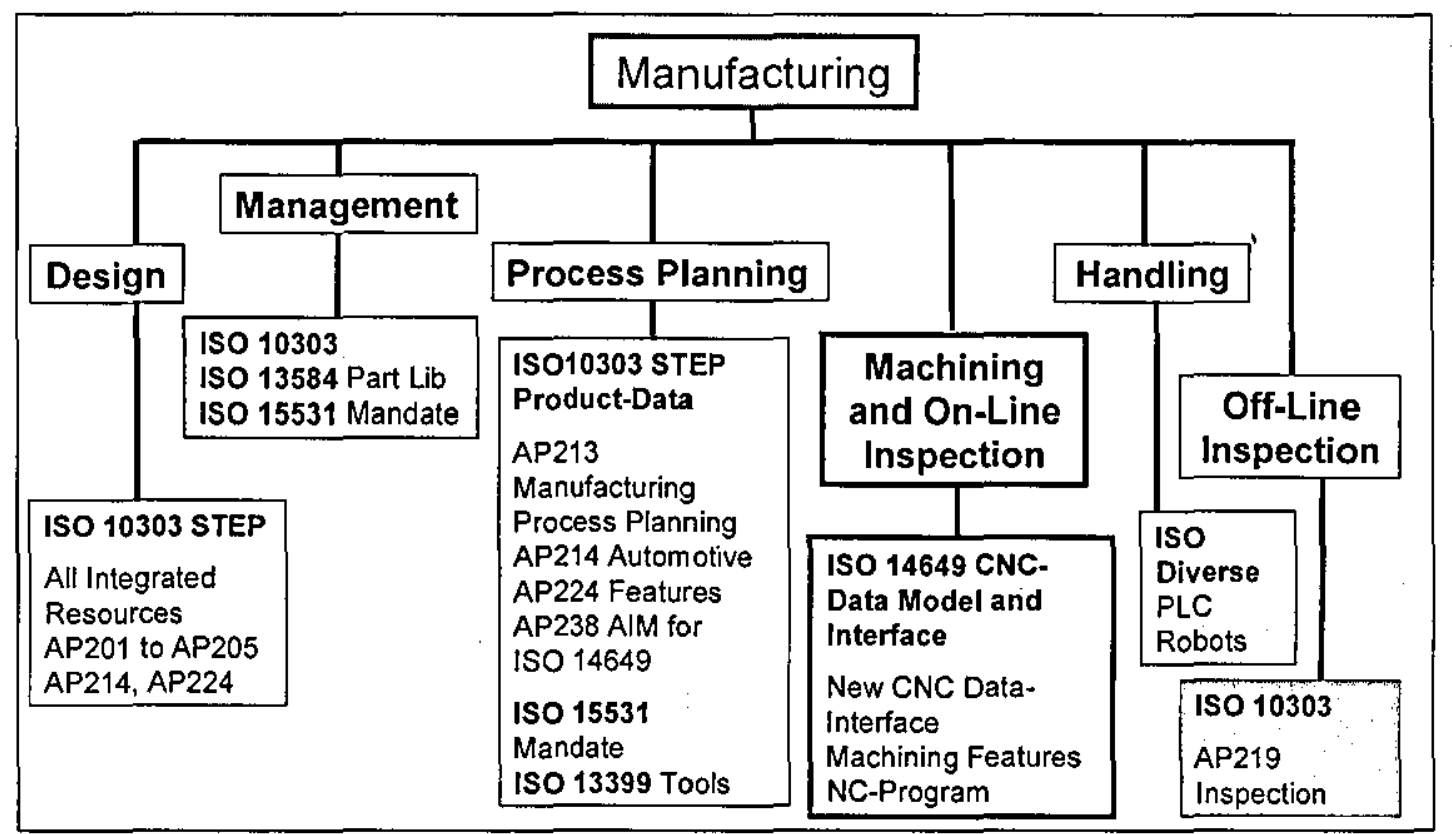

Figure 2.9 : Manufacturing activities and related standards (Glantschnig, 2001)

\subsubsection{STEP-NC Process Planning}

Suh et al (2004) have patented a method for automatically generating a part program for use in a STEP-NC (STEP-Compliant Data Interface for Numerical Controls) controller in a SFP (shop-floor programming) system based on an ISO 14649 data model. The method consists of several steps:

i. generating a geometric kernel data by interpreting a STEP physical file or an ISO 14649 part program;

ii. recognizing manufacturing features from the geometric kernel data

iii. setting a process plan according to the ISO 14649 on the basis of the manufacturing features;

iv. editing the process plan;

v. generating an ISO 14649 part program from the edited process plan;

vi. generating a tool path based on manufacturing feature information specified in the ISO 14649 part program; and

vii. verifying the produced tool path in a CNC (computer-based numerical control) apparatus. 


\subsubsection{STEP-NC Compliant CAD/CAM System}

This topic focuses on STEP-NC compliance, and the role it plays in closing the gap between design and manufacturing for a distributed and collaborative manufacturing environment. This new standard defines a new generation of NC programming language and is fully compliant with STEP. STEP-NC brings richer information to the $\mathrm{CNC}$ machine tools; hence intelligent machining and control are made possible. Its Web-enabled feature gives it an additional dimension in that distributed manufacturing can be readily supported.

\subsubsection{The Structure of STEP-NC}

The STEP-NC data model consists of two descriptions, the first is geometry describing manufacturing features and the second is the operations describing the process including working steps (Liu and Zhang, 2004). Four main groups of information are represented in the data model, namely: task description and part description, together with tool description and technology description. The first two items represent the scope of Part 10 of the standard and the latter two the machine and technological description for each process in Parts 11, 12, 13, etc. (Erdos and Xirouchakis, 2003). Figure 2.10 illustrates the basic structure and context of the STEP-NC data model.

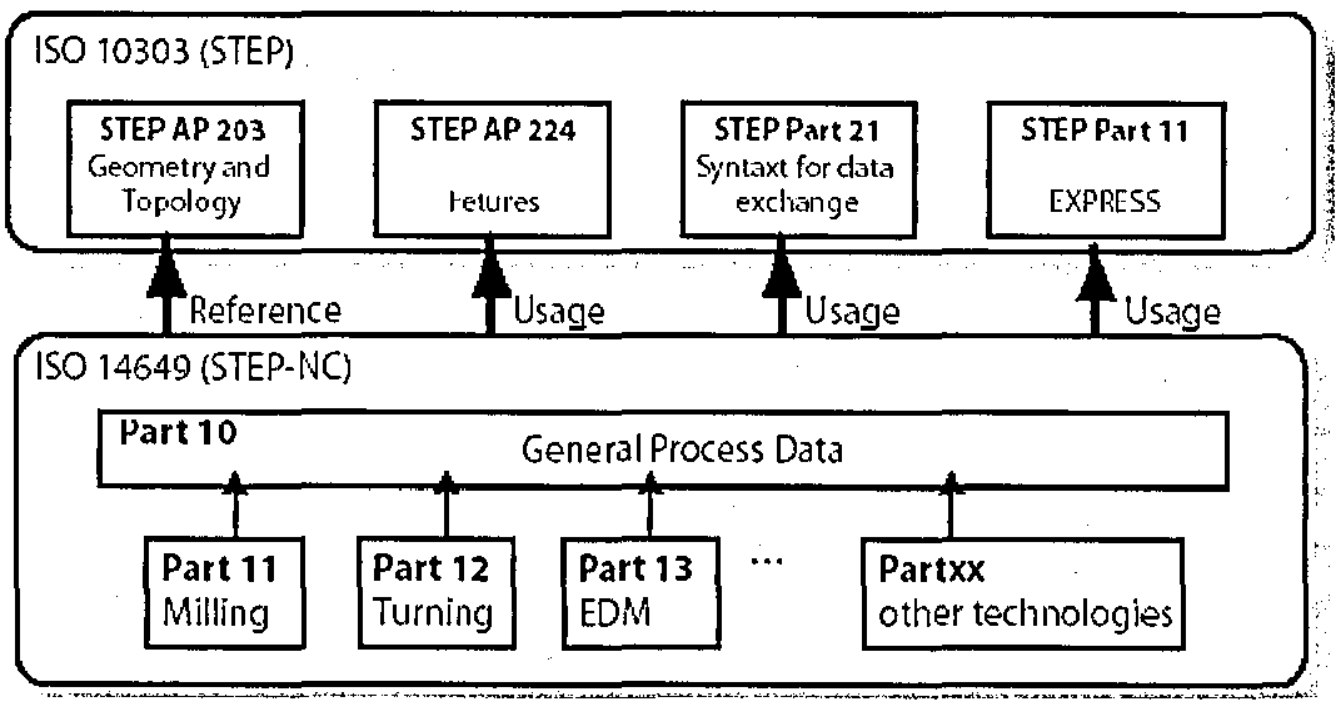

Figure 2.10 Basic structure and context of STEP-NC data model (Erdos and Xirouchakis, 2003) 


\subsubsection{The Physical File Format}

The physical file format is the basic structure and functionality of a typical STEP-NC part program (ISO, 2002; ISO, 2003b) and is summarised by Allen and $\mathrm{Xu}$ (Allen et al., 2003; Xu and $\mathrm{He}, 2004$ ).

i. Header: This is the first part of the program and describes the general information about the part program such as its filename, author, date, organization etc.

ii. Data: This is the second and main part of the program. These sections include of workplan and executables, technology description and geometry description as the three major parts in this section. Figure 2.11 represents the relationship among the three major parts.

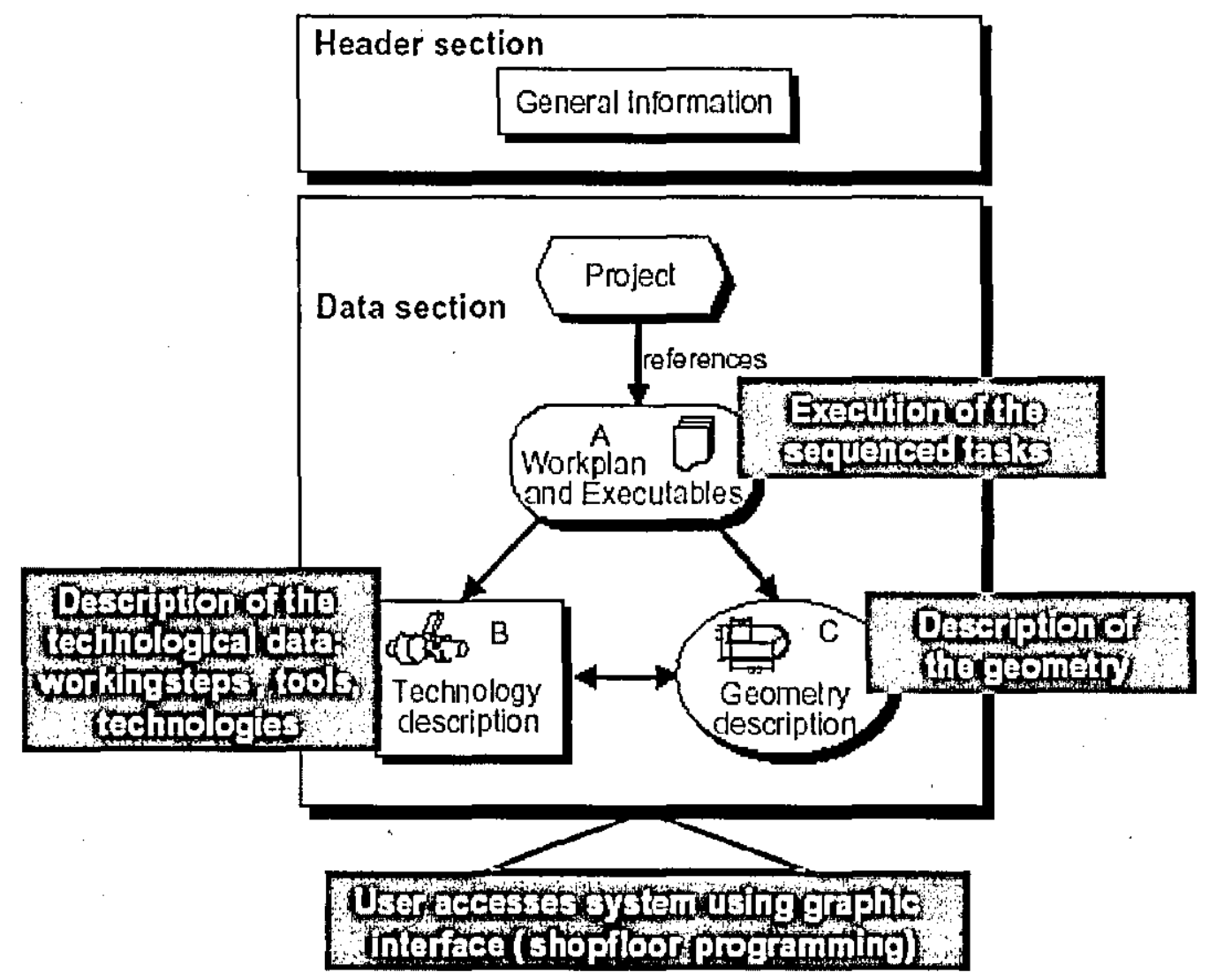

Figure 2.11 STEP-NC Physical File Structure (Muller, 2000a; Muller, 2000b) 
An extract from a STEP-NC file is shown as follows (Xu and He, 2004):

ISO-10303-21;

HEADER;

FILE DESCRIPTION(('A STEP-NC testing .le'),'l');

FILE part1.stp',\$,('AUMS'),(“),

'Prototype Mill',',','); NAME('sample contour',

FILE SCHEMA(('STEP-NC milling schema'));

ENDSEC;

DATA;

// Project and workplan

\#1=PROJECT('Contour',\#2,(\#3));

\#2=WORKPLAN('Work plan',(\#4),\$,\#5);

\#3=WORKPIECE('Workpiece',\#6,0.01,\$,

$\$$,\#8,());

// Working steps

\#4=MACHINING

WORKINGSTEP('Rough

Contour',\#13,\#16,\#17);

\#5=SETUP('main setup',\#44,\#48,(\#51));

\#6=MATERIAL('ST-50','Steel',(\#7));

\#7=PROPERTY PARAMETER

(' $\mathrm{E}=200000 \mathrm{~N} / \mathrm{mm}^{\wedge} 2$ ');

\#8=BLOCK('Block',\#9,260.000,210.000, $110.000)$;

// Geometric data

\#9=AXIS2 PLACEMENT 3D

('BLOCK',\#10,\#11,\#12);

// Manufacturing features

\#16=GENERAL OUTSIDE PROFILE

('Profile',\#3,(\#17),\#18,\#22,\$,\$,\$,\$,\#23,\$,

$\$)$;

// Operation data

\#17=SIDE

MILLING $\$$, \$,'Contour

pro.le', \#38,10.000,\#39,\#40,\#43,\$,\$,\$,20.0

00,5.000,0.000);
\#18=AXIS2 PLACEMENT 3D('Position of contour',

$\# 19$,\#20,\#21);

\#19=CARTESIAN POINT('Position of

$(40.000,90.000,100.000))$;

\#20=DIRECTION(",$(0.0,0.0,1.0))$;

\#21=DIRECTION(" , $(1.0,0.0,0.0))$;

\#22=TOLERANCED

LENGTH MEASURE $(20.000, \$, \$, \$)$;

\#23=COMPOSITE CURVE('Contour

Profile',

(\#24,\#25,\#56),.F.);

// Tool data

\#40=CUTTING TOOL('Endmill

$10 \mathrm{~mm}$,\#41,(),(50.000),50.000);

\#41=TAPERED

ENDMILL(,3,.RIGHT.,F.,\$,\$);

$\# 42=$ TOOL

DIMENSION $(10.000, \$, \$, \$, \$, \$)$;

// Machining technology

\#43=MILLING

TECHNOLOGY(\$,TCP.,\$,3.3333,\$,

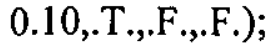

\#44=AXIS2

PLACEMENT

3D('Reference point to

Machine zero,\#45,\#46,\#47);

\#45=CARTESIAN

POINT(" ,(20.000,30.000,10.000));

\#56=COMPOSITE CURVE SEGMENT

(.CONTINUOUS.,.T.,\#57);

\#57=POLYLINE('Second cut of the contour',

ROUGH (\#29,\#30,\#31,\#32,\#33,\#27));

$\# 30, \# 31, \# 32, \# 33, \# 27$ );

ENDSEC;

END-ISO-10303-21; 


\subsection{Programming Standard Comparison}

Based on the literature reviewed a comparison on the use of STEP-NC and G-M codes for manual programming will be discussed in this section. Figure 2.12 summarises the comparison of both standards. Rosso has specified the comparison issues for STEP-NC and previous standards as shown in table 2.2 (Rosso-Jr, 2005).

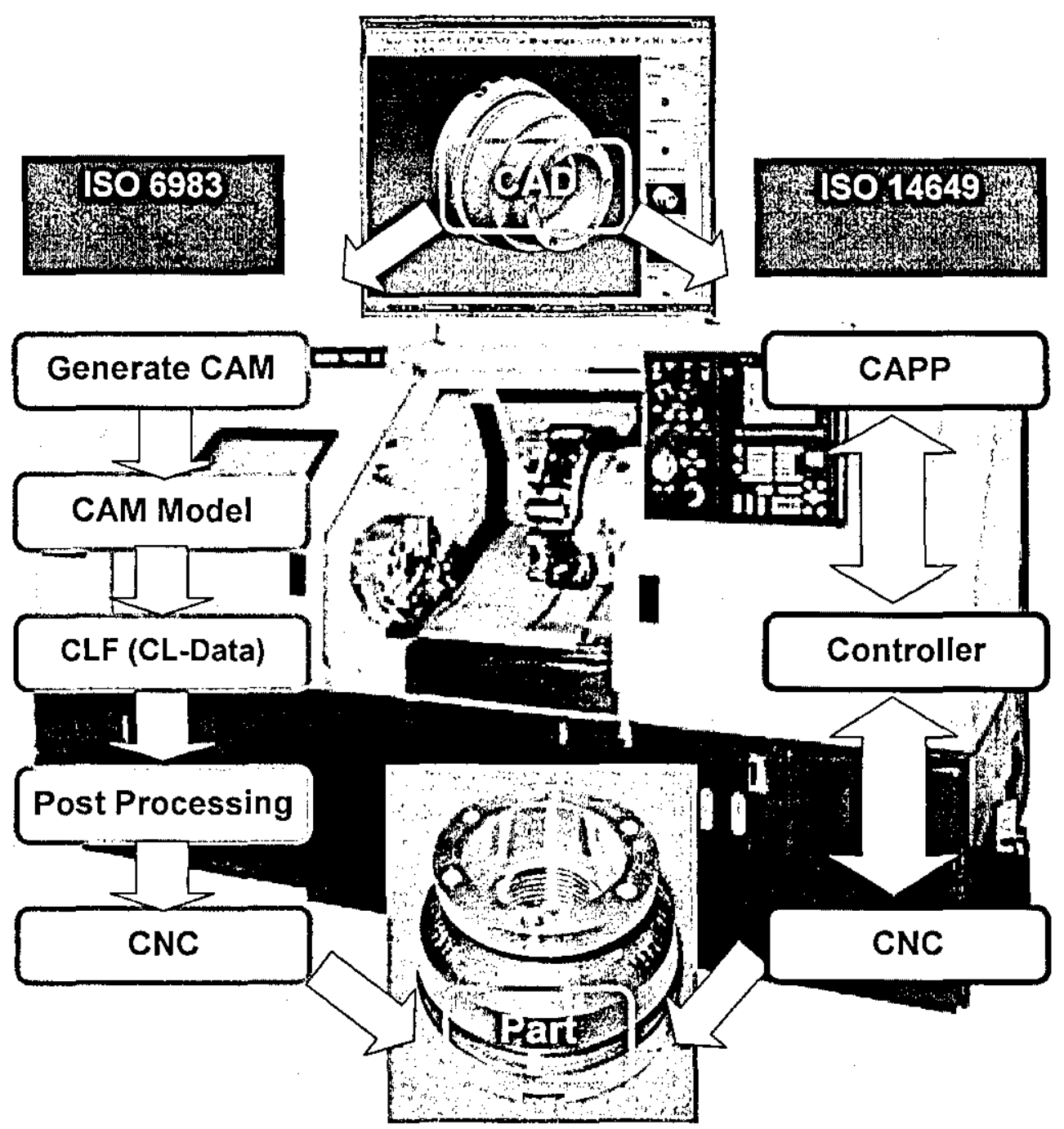

Figure 2.12 Comparison approach both standards 
Chapter 2

Table 2.2 Comparison issues for STEP-NC and previous standards (Rosso-Jr, 2005)

\begin{tabular}{|c|c|c|c|}
\hline $\begin{array}{l}\text { Comparison } \\
\text { issue }\end{array}$ & $S T E P-N C$ & $\begin{array}{l}\text { NC part } \\
\text { programming system }\end{array}$ & $\begin{array}{l}\text { Manual } \\
\text { programming }\end{array}$ \\
\hline $\begin{array}{l}\text { Programming } \\
\text { level }\end{array}$ & $\begin{array}{l}\text { Highest level } \\
\text { command }\end{array}$ & $\begin{array}{l}\text { Highest level } \\
\text { command }\end{array}$ & $\begin{array}{l}\text { Lowest level } \\
\text { command }\end{array}$ \\
\hline $\begin{array}{l}\text { Program } \\
\text { length }\end{array}$ & $\begin{array}{l}\text { Similar length to } \\
\text { manual part program, } \\
\text { but has different data }\end{array}$ & $\begin{array}{l}\text { Not really applicable } \\
\text { but data stored in } \\
\text { CAM system format } \\
\text { in software }\end{array}$ & $\begin{array}{l}\text { Efficient for } \\
\text { toolpaths when } \\
\text { combined with } \\
\text { canned cycle/ sub- } \\
\text { programs, can be } \\
\text { very long for 3D } \\
\text { parts }\end{array}$ \\
\hline $\begin{array}{l}\text { Part } \\
\text { description }\end{array}$ & $\begin{array}{l}\text { ISO standard using } \\
\text { geometric features }\end{array}$ & $\begin{array}{l}\text { Software specific } \\
\text { pseudo standard }\end{array}$ & $\begin{array}{l}\text { No part geometry in } \\
\text { code }\end{array}$ \\
\hline $\begin{array}{l}\text { Technological } \\
\text { description } \\
\text { e.g. tool } \\
\text { definition and } \\
\text { speeds }\end{array}$ & $\begin{array}{l}\text { Tool definition to ISO } \\
\text { standard \& cutting } \\
\text { tool parameters }\end{array}$ & $\begin{array}{l}\text { NC system holds } \\
\text { database of tools and } \\
\text { cutting parameters }\end{array}$ & $\begin{array}{l}\text { NC tool description } \\
\text { accept tool pocket or } \\
\text { tool assembly code }\end{array}$ \\
\hline Toolpaths & $\begin{array}{l}\text { Not described, left to } \\
\text { intelligent controller }\end{array}$ & $\begin{array}{l}\text { Toolpaths simulated } \\
\text { shown and output } \\
\text { through post } \\
\text { processor }\end{array}$ & $\begin{array}{l}\text { Toolpath information } \\
\text { based on tool centre } \\
\text { line }\end{array}$ \\
\hline Readability & $\begin{array}{l}\text { Easy to follow and } \\
\text { read major process } \\
\text { operations complex } \\
\text { data statements }\end{array}$ & $\begin{array}{l}\text { Easy to follow } \\
\text { operations and uses } \\
\text { dialogues and high } \\
\text { level language }\end{array}$ & $\begin{array}{l}\text { Lowest level } \\
\text { commands with } \\
\mathrm{XYZ} \text { and } \mathrm{G} \text { and } \mathrm{M} \\
\text { codes }\end{array}$ \\
\hline $\begin{array}{l}\text { Surface/ } 3 D \\
\text { capability }\end{array}$ & $\begin{array}{l}\text { Not defined as yet in } \\
\text { detail }\end{array}$ & $\begin{array}{l}\text { Based on 3D solid } \\
\text { geometry, surface } \\
\text { machining now } \\
\text { standard }\end{array}$ & $\begin{array}{l}\text { Possible but program } \\
\text { needs processing } \\
\text { from CAD/CAM } \\
\text { system }\end{array}$ \\
\hline $\begin{array}{l}\text { Machine tool } \\
\text { description }\end{array}$ & $\begin{array}{l}\text { Little definition of the } \\
\text { machine or its } \\
\text { intelligence }\end{array}$ & No definition & No definition \\
\hline
\end{tabular}


Feeney and Frechette (2002) have tested STEP-NC implementations and found some advantages;

i. A STEP-NC file contains all the data required to produce a part, therefore, manufacturing operations may be adjusted to maximize production efficiency.

ii. STEP-NC allows for complete safety checking because safety areas for fixtures can be defined as part of the setup.

iii. Documentation may be easily generated by the CAM or CNC system to show the state of the part before and after each working step.

iv. STEP-NC is easy to generate; specific tool paths need not be defined in advance.

v. A STEP-NC file is not machine-specific; the STEP-NC file can be manufactured on any machine that meets the tooling requirements.

Muller has reported that the benefits of the STEP-NC process chain are the availability of the whole product data management in every process part (Muller, 2004), so that for example the geometry data developed from CAD are used without modification down at the shop floor if they are feature based. In terms of cost saving for manufacturers, OMAC have reported at least two quantifiable points; i) purchasing power by implementing STEP-NC across the production lifecycle will provide enhanced purchasing power for acquiring factory assets. ii) Single source storage, because STEP-NC would enable a central repository for all machine parts production process information (OMAC, 2002).

The overall comparison can be made as the traditional CAD/CAM systems are aimed at simplifying the job of generating tool paths and the future CAD/CAM focuses more on a generic process plan and ignores the lower level of details. Future CNC controllers will have all the functionality of the remote workstation, being able to create and modify information at the machine itself. 


\subsection{Summary}

Based on the literature review, the evaluation of the topic of standardisation of manufacturing process can be summarised as:

i. An efficient method for data exchange; for more than three decades, a number of standards for data exchange have been created such as IGES, SET, PDES and other formats but only STEP has been recognised to represent the whole life cycle of a product (Rosso-Jr et al., 2004) due to success in transferring date between CAD systems. However it has failed to transfer product data from CAD to CAPP systems (Fidan, 2004).

ii. STEP is the most recent and promising standard for representing part data and a number of experimental product modelling systems have been developed based upon the STEP standard (Dutta et al., 1998).

iii. Interoperability issues such as different formats, different representations, different behaviour and incompatible content are issues in an information exchange transaction.

iv. Comparison of STEP-NC and previous standards shows that the data format is better structured information rather than the machine dependent format with no knowledge of the workpiece and machining tasks.

v. In terms of information, previous standards were based on "how-to-do" rather than "what-to-do".

vi. STEP-NC is close to a high level of information for generating process plans that consist of the description of machining tasks and machining requirements.

The next chapter discusses research applications using STEP-NC 
Chapter 3

\section{Chapter 3 - STEP-NC Research}

\subsection{Introduction}

This chapter provides a literature review of the STEP-NC compliant research around the world. The first part of this chapter focuses on projects based on STEP compliance followed by research and development in this area based on machining operations. This is followed by reviews of STEP-NC-Compliant systems for the manufacturing environment, focused on turning operations. The chapter concludes with discussion of the applications in this particular area.

\subsection{STEP-Compliant NC: Research Projects}

Since the late 90 s, many efforts have been made based on implementation of the STEP concept as the data interface between CAD/CAM and CNC. One of the pioneer projects, OPTIMAL (ESPRIT, 1997), proposed a new data interface framework for the milling process. STEP-NC has been developed as a result of several research projects carried out by companies and university institutes. In recent years there have been a number of projects involving STEP-NC based interoperable research and development for various $\mathrm{CNC}$ manufacturing processes. This chapter focuses on two projects, namely the Intelligent Manufacturing System (IMS) based on worldwide participants and Intelligent Manufacture for STEP-NC Compliant and Inspection, STEP compliant research work based at Loughborough University and the University of Bath. 


\subsubsection{The Intelligent Manufacturing System (IMS)}

In the second half of the 1990s an effort from the international community backed by ISO started the major change in the concept of NC programming, through an international intelligent manufacturing systems (IMS) programme as shown in figure 3.1 (Maeder et al., 2002). The STEP-based interface scheme was extended to 2.5D milling and other operations, such as turning and EDM in the subsequent European Project ESPRIT IV 29708 between 1999 and 2001 (Maeder et al., 2002).

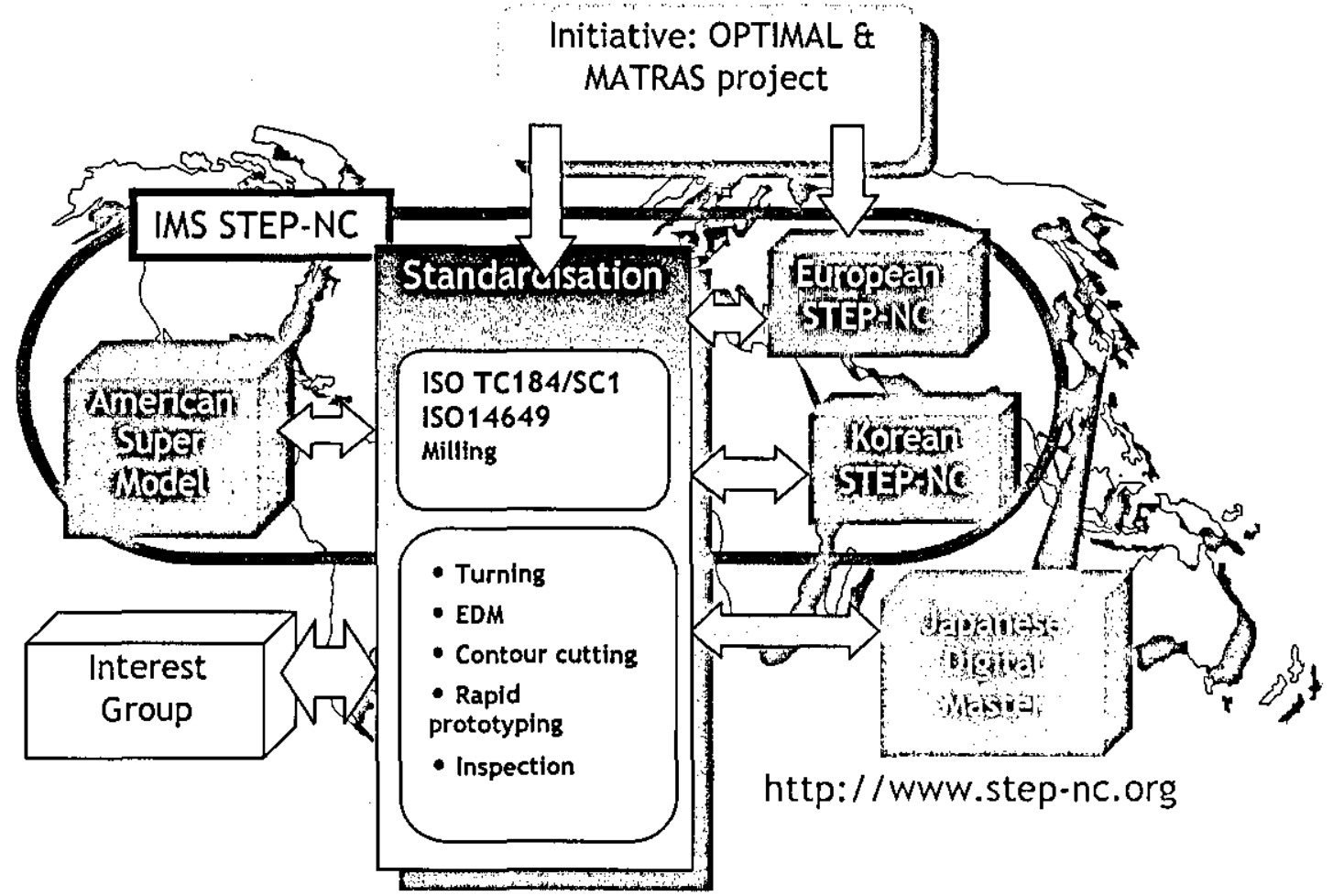

Figure 3.1 IMS Research Project (Maeder et al., 2002)

The IMS project was endorsed in November 2001 and it was a true international project with participants from four different regions (European Union, Korea, Switzerland and USA). Table 3.1 shows the participants and the distribution of the technological scope within each region. The participants included end users, academic institutions and manufacturers of CAD systems, controllers and machine tools. 
Chapter 3

Table 3.1: Participants and regions involved in the IMS project (Xu and He, 2004)

\begin{tabular}{|c|c|c|c|c|}
\hline Region & $E U$ & Switzerland & Korea & $\boldsymbol{U S A}$ \\
\hline \multirow{2}{*}{$\begin{array}{l}\text { Technologies } \\
\text { covered }\end{array}$} & Milling & & & \\
\hline & $\begin{array}{l}\text { Contour } \\
\text { cutting, } \\
\text { Turning \& } \\
\text { Inspection }\end{array}$ & $\begin{array}{l}\text { Wire/Sink } \\
\text { EDM }\end{array}$ & $\begin{array}{l}\text { Rapid } \\
\text { Prototyping }\end{array}$ & $\begin{array}{l}\text { AIM for Milling \& } \\
\text { Turning }\end{array}$ \\
\hline End-user & $\begin{array}{l}\text { * Daimler } \\
\text { Chrysler } \\
\text { * Volvo } \\
\text { * Franci (Italy) } \\
\text { * Progetti }\end{array}$ & $\begin{array}{l}\text { * Derendinger } \\
\text { * Wyss }\end{array}$ & * Samsung & $\begin{array}{l}\text { * IRB(including } \\
\text { * Boeing } \\
\text { * Lockheed Martin } \\
\text { * General Electric } \\
\text { * General } \\
\text { Dynamics, } \\
\text { General Motors, } \\
\text { etc.) }\end{array}$ \\
\hline $\begin{array}{l}\text { Machine tool } \\
\text { manufacturer }\end{array}$ & * CMS (Italy) & $\begin{array}{l}* \text { AGIE } \\
* \text { Starrag }\end{array}$ & & \\
\hline $\begin{array}{l}\text { Controller } \\
\text { manufacturer }\end{array}$ & $\begin{array}{l}\text { * Siemens } \\
\text { * OSAI (Italy) } \\
\text { * Fidia }\end{array}$ & & & \\
\hline $\begin{array}{l}\text { CAM } \\
\text { manufacturer }\end{array}$ & $\begin{array}{l}\text { * Open Mind } \\
* \text { Dassault }\end{array}$ & $\begin{array}{l}\text { * CAD } \\
\text { CAMation }\end{array}$ & * Cubictek & $\begin{array}{l}* \text { STEP Tools } \\
\text { * Gibbs \& } \\
\text { Associate } \\
\text { * BA Solutions } \\
\text { * Numerical } \\
\text { Control } \\
\text { Services }\end{array}$ \\
\hline $\begin{array}{l}\text { Research } \\
\text { institute }\end{array}$ & $\begin{array}{l}{ }^{*} \text { WZL } \\
\text { (RWTH } \\
\text { AACHEN) } \\
{ }^{*} \text { ISW } \\
\text { (University of } \\
\text { Stuttgart) } \\
{ }^{*} \text { KTH }\end{array}$ & $\begin{array}{l}* \text { EPFL } \\
* \text { EIG I-tech }\end{array}$ & $\begin{array}{l}\text { * ERCACI } \\
\text { * KIST } \\
\text { * NRL-SNT }\end{array}$ & $\begin{array}{l}\text { * Louisiana Centre } \\
\text { for } \\
\text { Manufacturing } \\
\text { Sciences } \\
\text { * Lawrence } \\
\text { Livermore } \\
\text { National } \\
\text { Laboratories }\end{array}$ \\
\hline Association & $\begin{array}{l}\text { * CECIMO } \\
\text { (Belgium) }\end{array}$ & * AMT & & $\begin{array}{l}\text { * NIST } \\
\text { * Department of } \\
\text { Energy } \\
\text { * Army's National } \\
\text { Automotive } \\
\text { Centre (NAC) }\end{array}$ \\
\hline
\end{tabular}


Chapter 3

\subsubsection{Intelligent Manufacture for STEP-NC Compliant Machining and} Inspection

This project was funded by the Engineering and Physical Sciences Research Council (EPSRC), is led by the AMST Centre at Loughborough University and University of Bath and started in May 2004. It explores the feasibility of utilising the information and database normally associated with CAD/CAM systems at the $\mathrm{CNC}$ machine tool. The research utilised and extended the standard for in-process measurement at the machine and also explored the application and integration of the STEP-NC standards between the CNC machine and a Co-ordinate Measuring Machine (CMM). A further major underlying theme of the research is the application of artificial intelligence to the CAPP/CAM/CNC process with the application of agent technology through the use of data mining. The project is supported by a number of industrial partners namely Renishaw, Siemens, LSC Group, Delcam and Rolls Royce (Newman et al., 2004).

The project has been undertaken in six work-packages (WP) including evaluation of the STEP-NC standards; development of STEP-NC compliant information models, development of CAx knowledge modelling with agent technology, specification development and implementation of a STEP-NC compliant CAPP/CAM/CNC facility and demonstrator and the evaluation of the STEP-NC prototype system. The project involved industrial collaboration (Newman et al., 2004);

i. Renishaw utilised the development of the STEP-NC standards for process control to develop a new product for measurement feedback from machine tools.

ii. The LSC Group utilised the integration of STEP-NC information with intelligent agent technology to enhance their LOCAM process planner which has been integrated with a CNC controller.

iii. Delcam utilised the STEP Compliant knowledge and information models to support the development of their Power Mill/ Inspect and Fixture products.

iv. Rolls Royce has the capability to evaluate the effectiveness of the 
STEP-NC approach through manufacture of their case study parts in the final stage of the research.

Research work at Loughborough University resulted in an agent based approach to STEP-NC CAD/CAM highlighted by Allen (Allen, 2003). Subsequently the information models for manufacture of asymmetric rotational components was the major challenge in implementing STEP-compliant CAD/CAM systems (Rosso-Jr et al., 2004). This was followed by Ho who concentrated on a STEP-NC Compliant CAx System for Wire Cutting EDM where the major contribution is the design of a STEP-NC compliant CAx system framework with product and manufacturing information models supporting the WEDM process chain (Ho et al., 2005). Finally Ali focused on the development of a STEP-NC Compliant Feature-based Inspection Framework for Prismatic Parts supported by STEP-Compliant product and manufacturing/inspection information models and the output of a feature based inspection plan (Ali et al., 2005).

\subsection{STEP-Compliant NC: Research and Development Systems}

STEP-NC has been developed as a result of several research projects carried out by companies and university institutes as shown in table 3.2 which is focused on machining and inspection. Recently a number of projects involving the areas of STEP-NC based interoperability and research and development for various CNC manufacturing processes have been started. 
Chapter 3

Table 3.2 STEP-NC Research from 2002-2007

\begin{tabular}{|c|c|c|c|c|}
\hline $\begin{array}{l}\text { Implementation } \\
\text { in machining }\end{array}$ & Milling operation & $\begin{array}{l}\text { Turning } \\
\text { operation }\end{array}$ & Wire EDM & Inspection \\
\hline 2002 & $\begin{array}{l}\text { (Feeney and } \\
\text { Frechette, 2002) } \\
\text { (Hardwick, 2002; } \\
\text { Lee and Bang, } \\
\text { 2002; Lewis, 2002) } \\
\text { (Newman et al., } \\
\text { 2002) } \\
\text { (Suh and Cheon, } \\
\text { 2002) } \\
\end{array}$ & $\begin{array}{l}\text { (Storr and } \\
\text { Heusinger, } \\
2002 \text { ) }\end{array}$ & - & - \\
\hline 2003 & $\begin{array}{l}\text { (Allen et al., 2003; } \\
\text { Hardwick, 2003; } \\
\text { Lee and Bang, } \\
\text { 2003) }\end{array}$ & $\begin{array}{l}\text { (Heusinger, } \\
2003 \text { ) } \\
\text { (Suh et al., } \\
2003 \text { ) }\end{array}$ & $\begin{array}{l}\text { (Erdos and } \\
\text { Xirouchakis } \\
\text { 2003) }\end{array}$ & - \\
\hline 2004 & $\begin{array}{l}\text { (Liu, 2004) } \\
\text { (Liu et al., 2004a) }\end{array}$ & $\begin{array}{l}\text { (Rosso-Jr et } \\
\text { al., 2004) } \\
\text { (Xu and } \\
\text { Wang, } \\
\text { 2004) }\end{array}$ & - & - \\
\hline 2005 & $\begin{array}{l}\text { (Allen et al., 2005) } \\
\text { (Nassehi et al., } \\
2005 \text { ) }\end{array}$ & $\begin{array}{l}\text { (Rosso-Jr, } \\
2005 \text { ) } \\
\text { (Xu et al., } \\
2005 \text { ) } \\
\text { (Yusof et } \\
\text { al., 2006a) }\end{array}$ & $\begin{array}{l}\text { (Ho et al., } \\
2005 \text { ) }\end{array}$ & (Ali, 2005) \\
\hline 2006 & $\begin{array}{l}\text { (Hardwick and } \\
\text { Loffredo, 2006) } \\
\text { (Kramer et al., } \\
\text { 2006) } \\
\text { (Lee et al., 2006) } \\
\text { (Liu et al., 2007) } \\
\text { (Nassehi et al., } \\
\text { 2006a) } \\
\text { (Xu et al., 2006b; } \\
\text { Yamada et al., } \\
\text { 2006) } \\
\text { (Zhang et al., 2006) }\end{array}$ & $\begin{array}{l}\text { (Heusinger } \\
\text { et al., 2006) } \\
\text { (Choi et al., } \\
\text { 2006; Suh et } \\
\text { al., 2006) } \\
\text { (Suh et al., } \\
\text { 2006) } \\
\text { (Yusof et } \\
\text { al., 2006b) }\end{array}$ & $\begin{array}{l}\text { (Sokolov et } \\
\text { al., 2006) }\end{array}$ & $\begin{array}{l}\text { (Brecher et } \\
\text { al., 2006) }\end{array}$ \\
\hline 2007 & $\begin{array}{l}\text { (Amaitik and Kilic, } \\
2007 \text { ) } \\
\text { (Liu et al., 2007) } \\
\text { (Kumar et al., } \\
\text { 2007) }\end{array}$ & $\begin{array}{l}\text { (Shin et al., } \\
2007 \text { ) } \\
\text { (Yusof et } \\
\text { al., 2007) }\end{array}$ & - & - \\
\hline
\end{tabular}




\subsubsection{Review of STEP-Compliant Manufacturing for Milling Machining}

Overall research activities in specific areas of STEP-NC based on manufacturing technology and processes focus more on milling rather than turning activities, due to ISO 14649, Part 11 for milling operation being established before Part 12 (Turning). Table 3.2 clearly shows that research activities in milling operations are more common than turning in the last five years. Work from Shimamura, is recognised as one of the earliest pieces of research to address an alternative for enhancing the capability of the existing $\mathrm{NC}$ machines economically using a PC-based retrofitting scheme for the manufacture of free form surfaces (Shimamura et al., 1996). In 2002, research and development in terms of manufacturing technology and processes began with a proposal for the conceptual framework for designing and implementing an intelligent CNC system by Suh and Sheon (Suh and Cheon, 2002), followed by Hardwick providing the first outlook on STEP-NC compliant manufacturing (Hardwick, 2002). Lee and Bang have successfully developed and built a five-axis milling machine that is run by STEPNC in XML (Lee and Bang, 2002) and another prototype system has been proposed by Newman et al for a STEP-compliant CAD/CAM system based on one of these frameworks using the new ISO 14649 standard for milling components (Newman et al., 2002). Finally test and validation methods have been proposed for testing data for numerical control (Feeney and Frechette, 2002). It is noticeable that in 2006 researchers were extremely focused on this particular area, and details can be found a special issue edition of the International Journal of Computer Integrated Manufacturing (IJCIM) for STEP-Compliant Process Planning and Manufacturing (Xu et al., 2006a).

Kumar introduced a STEP-compliant framework that makes use of selflearning algorithms that enable the manufacturing system to learn from previous data and results in error elimination and consistent quality products. It has been tested and certified for pocket and hole features for milling (Kumar et al., 2007). The latest achievement in 2007 is the successful development of a system called ST-FeatCAPP for prismatic parts based on ISO 14649 by (Amaitik and Kilic, 2007). The system maps a STEP AP224 XML data file, without using a complex feature 
recognition process, and produces the corresponding machining operations to generate the process plan and corresponding STEP-NC in XML format. Liu et al. also proposed a NC programming system for prismatic parts to be machined using STEP-NC machine tools, and the system consisted of three functional modules, namely i) a feature-based modeller, ii) a process planner and iii) a part program generator. The system can read the STEP-NC file and calculate the toolpath automatically compared to current systems that only produce low level control information (Liu et al., 2007)

\subsubsection{Review of STEP-Compliant Manufacturing for Turning}

One of the aims for the next generation of $\mathrm{CNC}$ machines is to be interoperable and adaptable so that they can respond quickly to changes in market demand and the manufacturing needs of customized products (Xu et al., 2006a). As part of this, 2006 was a time when researchers were particularly focused on proposing a framework for turning. Most of the researchers proposed prototype systems to support data interoperability between the various CAx systems based on ISO standard 14649 that provided the first data exchange format used in the operation of NC machines (see table 3.3). Among these systems, G2STEP is the latest system to cover the machine functioning from pre-processor to STEP-NC part program generation including part program verification (Shin et al., 2007). This development of a future manufacturing platform to enable different processes and capability such as milling applications, multi-axis and complex components as the basis of the integration of $\mathrm{CAD} / \mathrm{CAPP} / \mathrm{CAM}$ and $\mathrm{CNC}$ will be a major research task for years to come. 
Tables 3.3: Summary review of STEP-Compliant manufacturing for Turning Operations

\begin{tabular}{|c|c|}
\hline Author(s) & Concerns \\
\hline $\begin{array}{l}\mathrm{Xu} \text { and Wang, } \\
2004\end{array}$ & $\begin{array}{l}\text { Developed a STEP-NC Converter and a retrofitted CNC } \\
\text { lathe realizing a G-code free machining scenario }\end{array}$ \\
\hline Wei et al., 2005 & $\begin{array}{l}\text { Proposed a framework for a CNC turning system based on } \\
\text { STEP-NC with eighteen functional modules, involved in the } \\
\text { software-based framework of a STEP-NC control system. } \\
\text { As a result, this paper shows the high potential to aid the } \\
\text { development of new CNC turning systems. }\end{array}$ \\
\hline Shin et al., 2006 & $\begin{array}{l}\text { Presented development of a prototype G2STEP system to } \\
\text { convert G-codes to a STEP-NC file. }\end{array}$ \\
\hline Choi et al., 2006 & $\begin{array}{l}\text { Introduced the development process for TurnSTEP using } \\
\text { structured and object oriented methodology to provide a } \\
\text { distributed architecture for e-manufacturing. }\end{array}$ \\
\hline Suh et al., 2006 & $\begin{array}{l}\text { Presents a STEP-CNC system for turning, named } \\
\text { TurnSTEP, and demonstrated the potential and power of } \\
\text { STEP-NC based CNC systems compared with conventional } \\
\text { CNC systems }\end{array}$ \\
\hline $\begin{array}{l}\text { Heusinger et al., } \\
2006\end{array}$ & $\begin{array}{l}\text { Presented a prototype methodology using technology based } \\
\text { on ISO14649-12 for implementing a standardised CAx } \\
\text { process chain for rotational asymmetric parts. }\end{array}$ \\
\hline Yusof et al., 2006 & $\begin{array}{l}\text { Presents the current CAPP and CAM systems related to } \\
\text { STEP-NC created by other researchers and the development } \\
\text { of a STEP-NC compliant CAD /CAPP /CAM system. }\end{array}$ \\
\hline
\end{tabular}

\subsection{Review of STEP-Compliant Systems}

One of the aims for the next generation of $\mathrm{CNC}$ machines is to be interoperable and adaptable so that they can respond quickly to changes in market 
demand and the manufacturing needs of customized products ( $\mathrm{Xu}$ et al., 2006a). The changing business environment over the past decades including globalisation resulted in the standards ISO 10303 and ISO 14649 (STEP and STEP-NC) being introduced to solve the interoperability issues. For the time being many obstacles come from software/hardware vendors as the current approaches give them many opportunities to maintain their market, but the new standards can provide the platform for the future of global interoperable manufacturing (Newman et al., 2007).

The Shop-floor Programming System (SFPS) introduced by Suh is the first system fully compliant with ISO 14649 (Suh et al., 2003) and to date, only this system has been patented (US patent references; 6400998, 65112961, 6556879, 6650960 and 6671571). SFPS and other systems related to STEP compliance that have been developed by academia all over the world are shown in table 3.4.

Table 3.4 Review of STEP-Compliant systems

\begin{tabular}{|l|l|l|l|l|}
\hline No & \multicolumn{1}{|c|}{ Systems } & \multicolumn{1}{|c|}{ Input } & \multicolumn{1}{c|}{ Output } & Domain \\
\hline 1 & $\begin{array}{l}\text { SFPS } \\
\text { (Milling) (Suh et } \\
\text { al., 2003) }\end{array}$ & $\begin{array}{l}\text { STEP } \\
\text { AP203 \& } \\
\text { AP214 }\end{array}$ & $\begin{array}{l}\text { Part program } \\
\text { physical file } \\
\text { (text) }\end{array}$ & Prismatic \\
\hline 2 & $\begin{array}{l}\text { STEPTurn } \\
\text { (Heusinger et al., } \\
\text { 2006; Xu, 2006) }\end{array}$ & $\begin{array}{l}\text { STEP } \\
\text { AP203 }\end{array}$ & $\begin{array}{l}\text { Part program } \\
\text { physical file } \\
\text { (text) }\end{array}$ & Rotational \\
\hline 3 & $\begin{array}{l}\text { TurnSTEP (Choi } \\
\text { et al., 2006; Suh et } \\
\text { al., 2006) }\end{array}$ & STEP AP & $\begin{array}{l}\text { ISO 14649 } \\
\text { physical file and } \\
\text { extensible mark- } \\
\text { up language } \\
\text { (XML) }\end{array}$ & Rotational \\
& & & & \\
\hline
\end{tabular}


Table 3.4 Review of STEP-Compliant systems (continued)

\begin{tabular}{|l|l|l|l|l|}
\hline No & \multicolumn{1}{|c|}{ Systems } & \multicolumn{1}{|c|}{ Input } & \multicolumn{1}{|c|}{ Output } & Domain \\
\hline 4 & $\begin{array}{l}\text { G-Code Free for } \\
\text { lathe (Xu and } \\
\text { Wang, 2004; Xu et } \\
\text { al., 2005) }\end{array}$ & $\begin{array}{l}\text { STEP AP } \\
203\end{array}$ & $\begin{array}{l}\text { Native CNC } \\
\text { language } \\
\text { program }\end{array}$ & Rotational \\
\hline 5 & $\begin{array}{l}\text { G2STEP } \\
\text { (2-axis turning } \\
\text { machining) (Shin et } \\
\text { al., 2007) }\end{array}$ & G-codes & $\begin{array}{l}\text { STEP-NC part } \\
\text { program }\end{array}$ & Rotational \\
\hline
\end{tabular}

\subsubsection{Shop-Floor Programming System (SFPS)}

The Shop-floor Programming System (SFPS) has been developed by researchers from POSTECH, South Korea as a computer assisted part programming tool capable of interfacing with a new CNC controller called STEP-CNC (STEPcompliant CNC) (Suh et al., 2003). This system has been proposed for prismatic components, as shown in figure 3.2, for generating ISO 14649 part programs based on the ISO documents such as ISO 14649 Parts 10, 11, 111 and ISO 10303 Parts 21, 22, 23, 42, 203 and 224. Suh et al., provided a useful definition of architecture and functionality including (Suh et al., 2003);

i. Full compliance with ISO 14649 and STEP APs. This is the fundamental requirement for SFPS for STEP-CNC.

ii. Feature recognition/mapping capability. An ISO 14649 part program is depicted with respect to the machining features. If the part geometry is given by AP203 feature recognition (mapping) is required for SFPS before proceeding to the process planning procedure.

iii. Database structure for STEP interface. The database of SFPS is structured such that it can interface with other STEP databases such as a 
STEP-repository storing all information, using a complete suite of STEP schema.

iv. Accommodation of conventional CNC. As mentioned earlier, there are three types of STEP-CNC depending on how ISO 14649 is interfaced with CNC. The first type uses conventional CNC based on ISO 6983 via post-processing. To accommodate conventional CNC, SFPS should be designed to output in the form of ISO 6983 as well as ISO 14649.

v. Editing capability. SFPS is also used for editing existing ISO 14649 part programs. For such a purpose, SFPS should be able to read (interpret) the ISO 14649 part program, and check the logical and syntax errors in it.

vi. Human interface. For accuracy and efficiency of part programming, SFPS should be designed to be user friendly. A graphic user interface and diagnostic messages together with visual verification are necessary.

vii. Process sequence editor. Conventionally, the process sequence is given in a linear (sequential) fashion. In practice, however, there often exist situations where the process sequence is not necessarily linear. In ISO 14649 , the sequence of workingsteps (program_structure) can be given in a non-linear fashion. This is to give flexibility to $\mathrm{CNC}$ in executing the tasks. However, programming a non-linear process plan is not easy for the programmer, and hence a process sequence editor should be provided by SFPS.

viii. Optimization/knowledge-based system. Completion of an ISO 14649 part program requires technical information. Even if a push-button interface is used, specifying all the entries may be cumbersome. Thus, minimization of input and provision of optimized values (cutting conditions, tool selections) are necessary using knowledge-based algorithms and technical databases. 


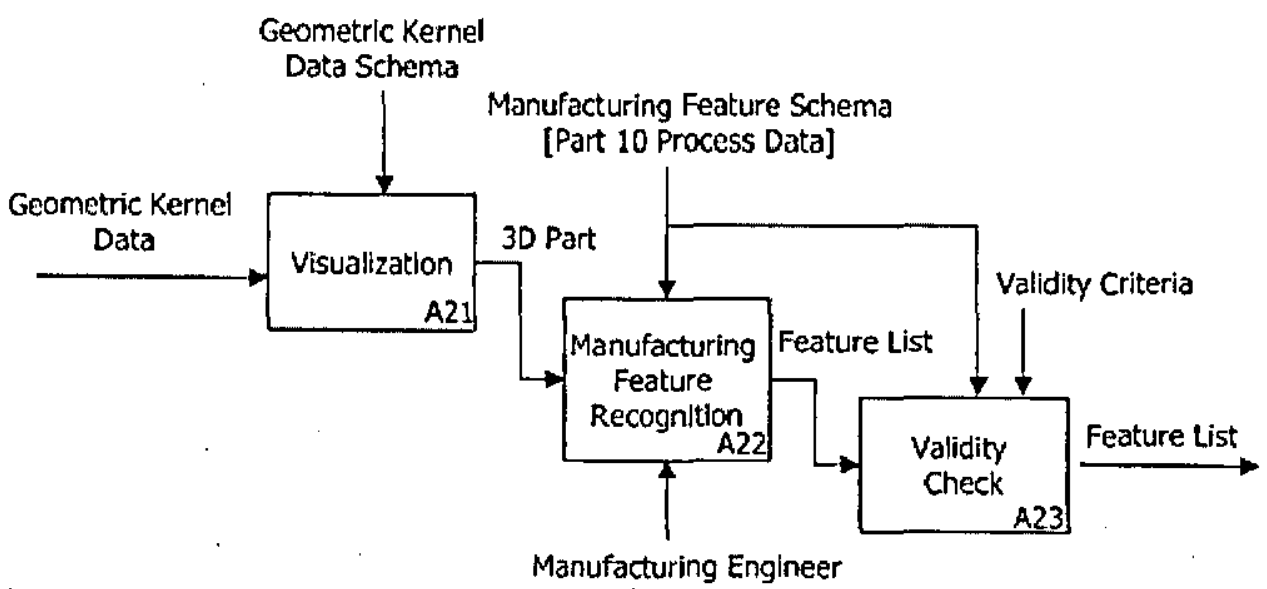

Figure 3.2 Machining feature recognition for SFPS (Suh et al., 2003)

SFPS provides a method for generating a part program for a STEP-NC system based on seven steps (Suh et al., 2004);

i. generating geometric kernel data by interpreting a STEP physical file or an ISO 14649 part program

ii. recognizing manufacturing features from the geometric kernel data

iii. setting a process plan according to the ISO 14649 on the basis of the manufacturing features

iv. editing the process plan

v. generating an ISO 14649 part program from the edited process plan as shown in figure 3.3 .

vi. generating a tool path based on manufacturing feature information specified in the ISO 14649 part program; and

vii. verifying the produced tool path in a $\mathrm{CNC}$ (computer-based numerical control) apparatus. 


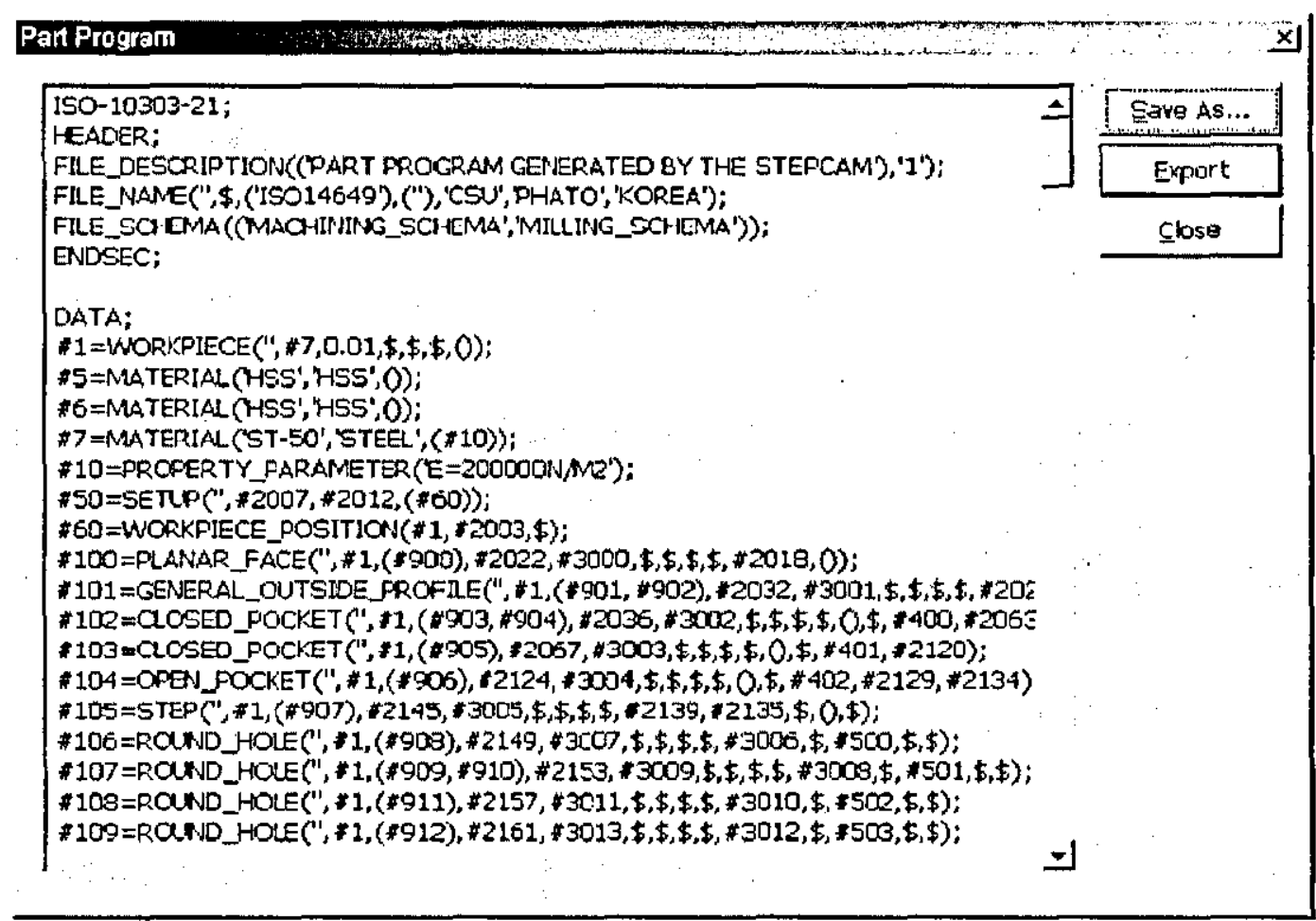

Figure 3.3 Generated ISO Part Program from SFPS (Suh et al., 2003)

SFPS is a pilot system created by one of the ISO 14649 authors during the time when ISO 14649 was at the draft and final draft for ISO documentation and had been presented to the ISO 14649 committee. SFPS is recognised as one of the pioneer systems to have successfully generated a part program from a design file format in either AP 203 or AP 214 (Suh et al., 2002; Suh et al., 2003; Xu et al., 2005). This pilot system is becoming the basic platform for future improvement to milling operations. It also provides a possible method that could be applicable to turning operations.

\subsubsection{STEPTurn}

STEPTurn has been developed by researchers from the Institute for Control Engineering of Machine Tools and Manufacturing Units at the University of Stuttgart (ISW), Germany. ISW adopted the STEP and STEP-NC standards for 
turned parts (Heusinger et al., 2006; Xu, 2006). STEPTurn is a CAPP system bridging the gap between CAD and CAM as shown in figure 3.4 and it reads geometry data firstly from a STEP AP-203 Part 21 file, and displays the part geometry and performs normal process-planning tasks such as feature recognition and Workingstep sequencing in order to generate a STEP-NC physical file (Heusinger et al., 2006; $\mathrm{Xu}, 2006$ ). According to Heusinger, the stages in using STEPTurn are (Heusinger, 2001); i) Input of AP 203 file, ii) Display of the workpiece, iii) Generation of operations, strategies and technology, iv) Definition of the workplan and v) Output of the STEP-NC program.

ShopTurn is used as the conversion data format of Siemens which is the input format to generate tool paths for the Siemens $840 \mathrm{D} \mathrm{NC}$ controller of the Boehringer machine tool as shown in figure 3.5 (Heusinger et al., 2006). The data filling the shop-floor-oriented feature dialogs was mapped onto the internal STEPNC data representation. After definition of features and their assignment to setups, operations were generated automatically. Where the operations have to be adapted to any other purpose, the operation data may be modified in operation dialogs (Muller, 2004). 
Chapter 3

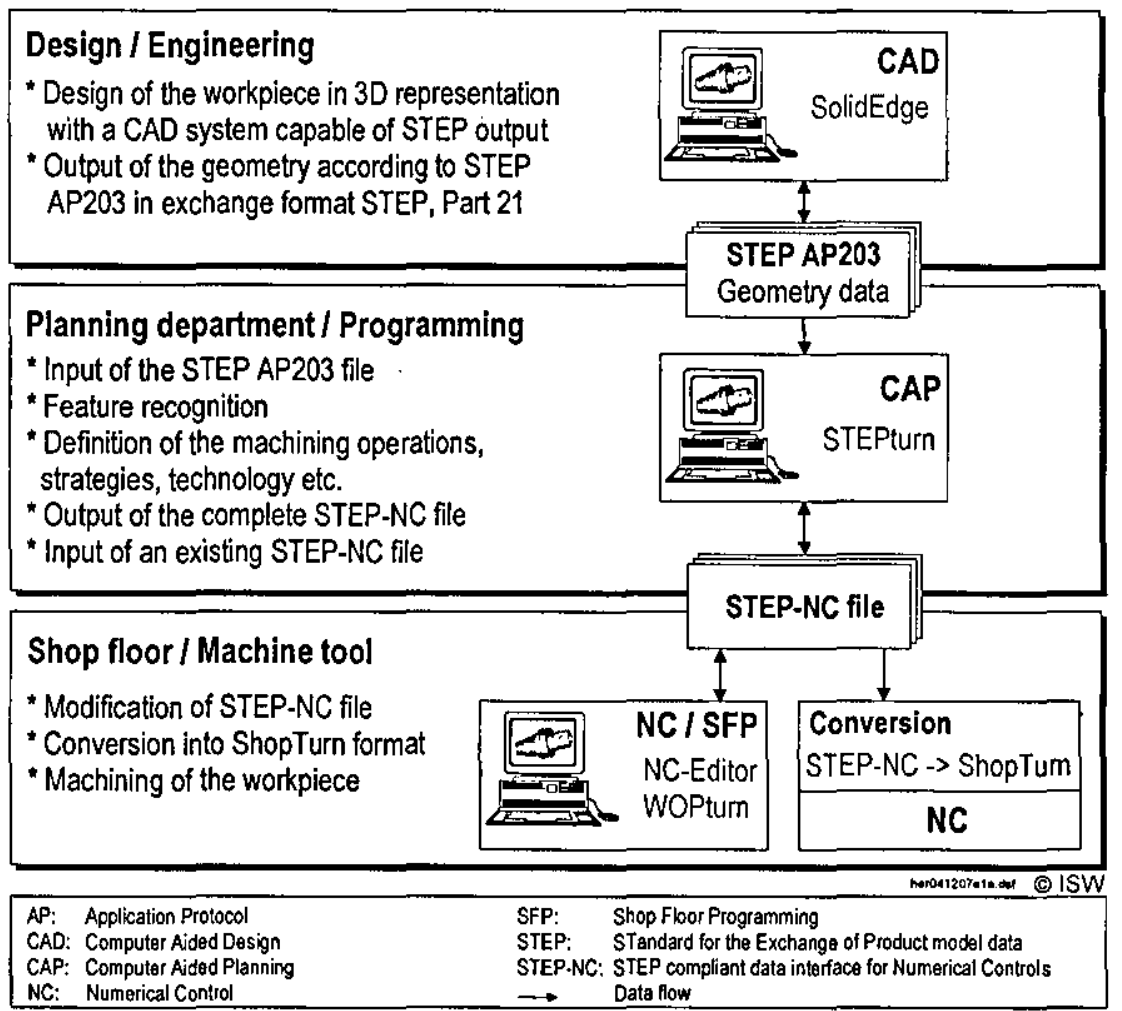

Figure 3.4 Implemented process chain for turning (Heusinger et al., 2006)

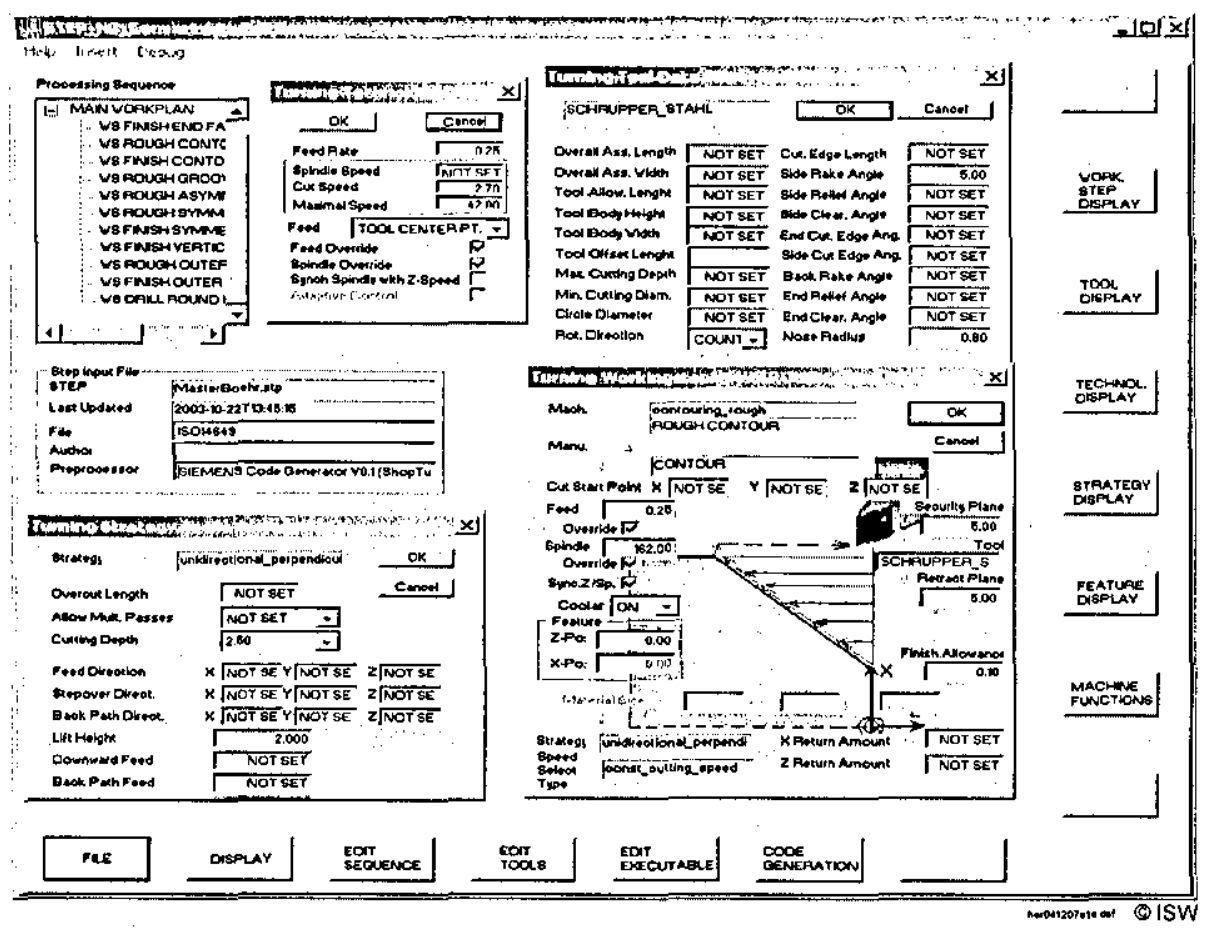

Figure 3.5 Conversion tool for translating STEP-NC to ShopTurn format

(Heusinger et al., 2006) 


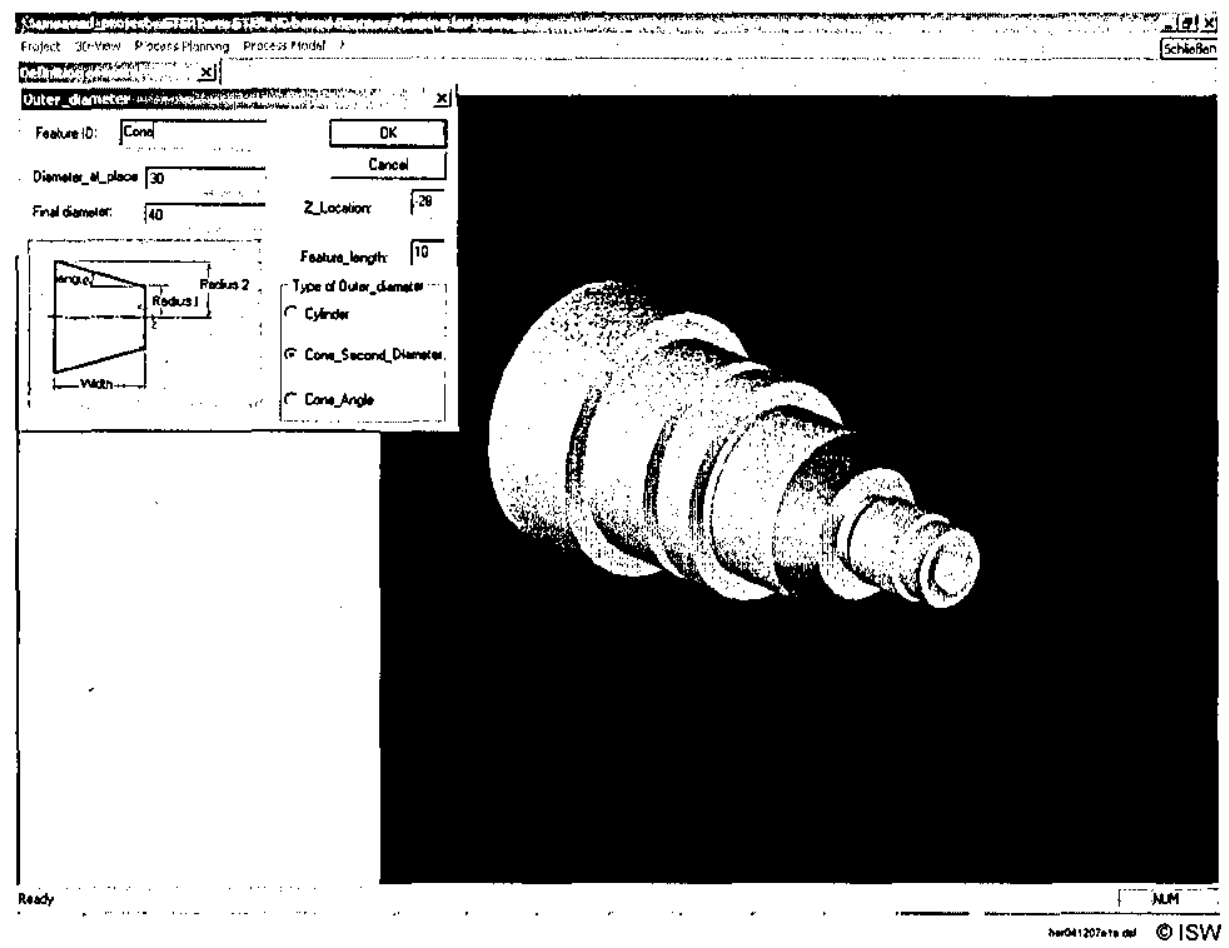

Figure 3.6 Prototypical CAP system: STEPTurn (Heusinger et al., 2006)

So far STEPTurn has been tested only for simple workingsteps for machining cylinders and cones using standard turning machines as shown in figure 3.6 ( $\mathrm{z}$ and $\mathrm{x}$ axis only) and does not really represent the capability of the proposed system. As stated by the researchers the prototype system needs to be extended for mill-turn components and to produce output in XML schemas (Heusinger et al., 2006). As clearly presented in the Heusinger paper STEPTurn only works on a specific machine (Heusinger et al., 2006).

\subsubsection{TurnSTEP}

TurnSTEP is claimed by Choi to be fully compliant with ISO 14649 and suitable for e-manufacturing (Choi et al., 2006; Suh et al., 2006). TurnSTEP has been developed using three sub-systems namely; i) CGS (Code Generating System) to generate neutral independent STEP-NC code based on ISO 14649, ii) CES (Code Editing System), to edit or customise machine tool data to be used for execution of 
STEP-NC code and iii) ACS (Autonomous Control System) as shown in figure 3.7 (Choi et al., 2006; Suh et al., 2006). In terms of turning machining, TurnSTEP is one of the earliest systems to have been developed for compliance with STEP-NC and supported by XML schema. From the design perspective this system needs (Choi et al., 2006);

i. to provide a distributed architecture for e-manufacturing;

ii. to support intelligent and autonomous execution of NC machines by fully utilizing rich STEP-NC information;

iii. to optimize the machining sequence of a target manufacturing process;

iv. to support automated and interactive generation of a process plan utilizing feature recognition, alternative generation, process sequence, cutting condition, etc;

v. to provide a variety of data interfaces for e-manufacturing including physical files and extensible markup language (XML) translation capability.

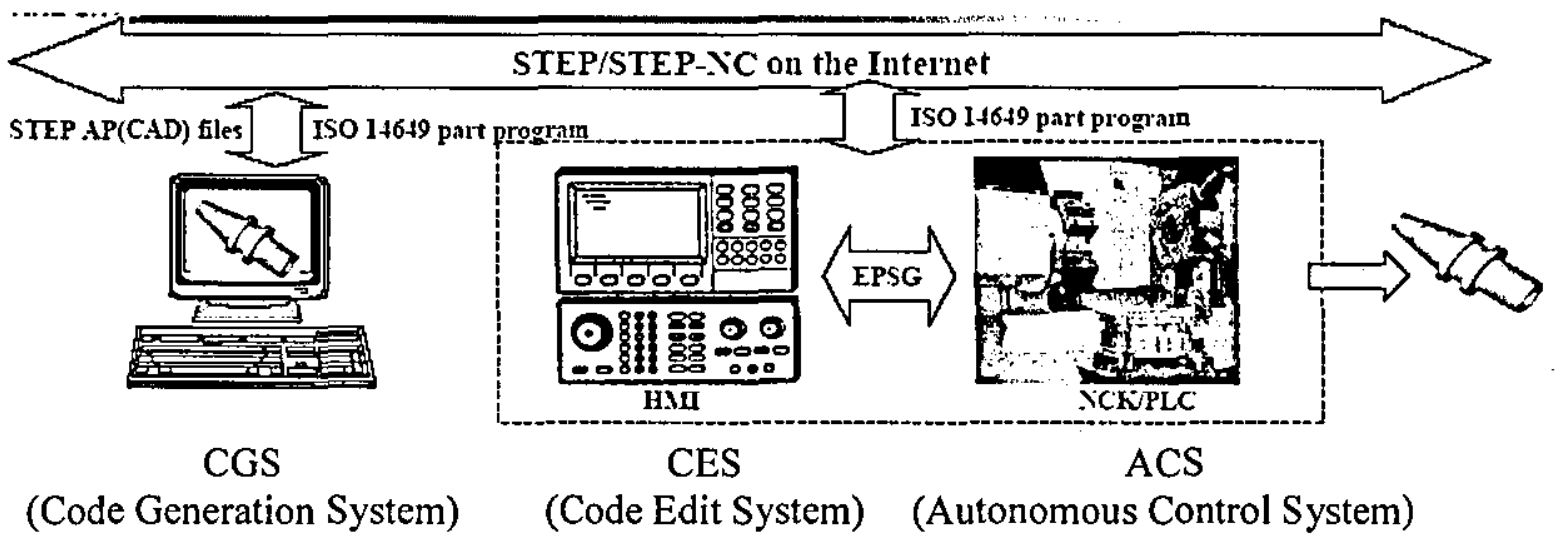

Figure 3.7 Three subsystems of the TurnSTEP (Choi et al., 2006)

TurnSTEP uses input geometry in an AP 203 format file to convert geometry information to an internal data form and map it to machining features by producing the machine independent format process planning (Suh et al., 2006). TurnSTEP also allows the operators to edit existing files using a user interface and the output can be stored by as a nonlinear process plan graph. This system has been 
tested using the simple example component in ISO 14649 Part 12 with standard turning operations. It loads an AP 203 file from a CAD system via the internet and visualises the part geometry. The blank part material is then defined, followed by determination of the number of set-ups and finally it generates a virtual machining simulation and machines the part (Suh et al., 2006)

\subsubsection{G-Code Free Lathe}

This STEP-compliant CAD/CAPP/CAM/CNC system has been developed in the Manufacturing Systems Group at the University of Auckland, New Zealand and was aimed at making product data interchangeable, product information flow seamless and a system that is independent of any CAD/CAM system (Xu and Wang, 2004). The proposed system has two parts i) retrofitting an existing CNC lathe and ii) a STEP CNC converter that is run with four phases; i) generation of generic STEP-NC programs, ii) generation of a native STEP-NC program, iii) generation of a native $\mathrm{CNC}$ language file and iv) execution of the process plan on a CNC machine as shown in figure 3.8 ( $\mathrm{Xu}$ and Wang, 2004). Xu describes how the low level language rather then G-codes is interpreted by STEP-NC into this axis movement language by using a $6 \mathrm{~K}$ program and passed on to the retrofitted lathe $(\mathrm{Xu}$ and Wang, 2004; Xu, 2006). 


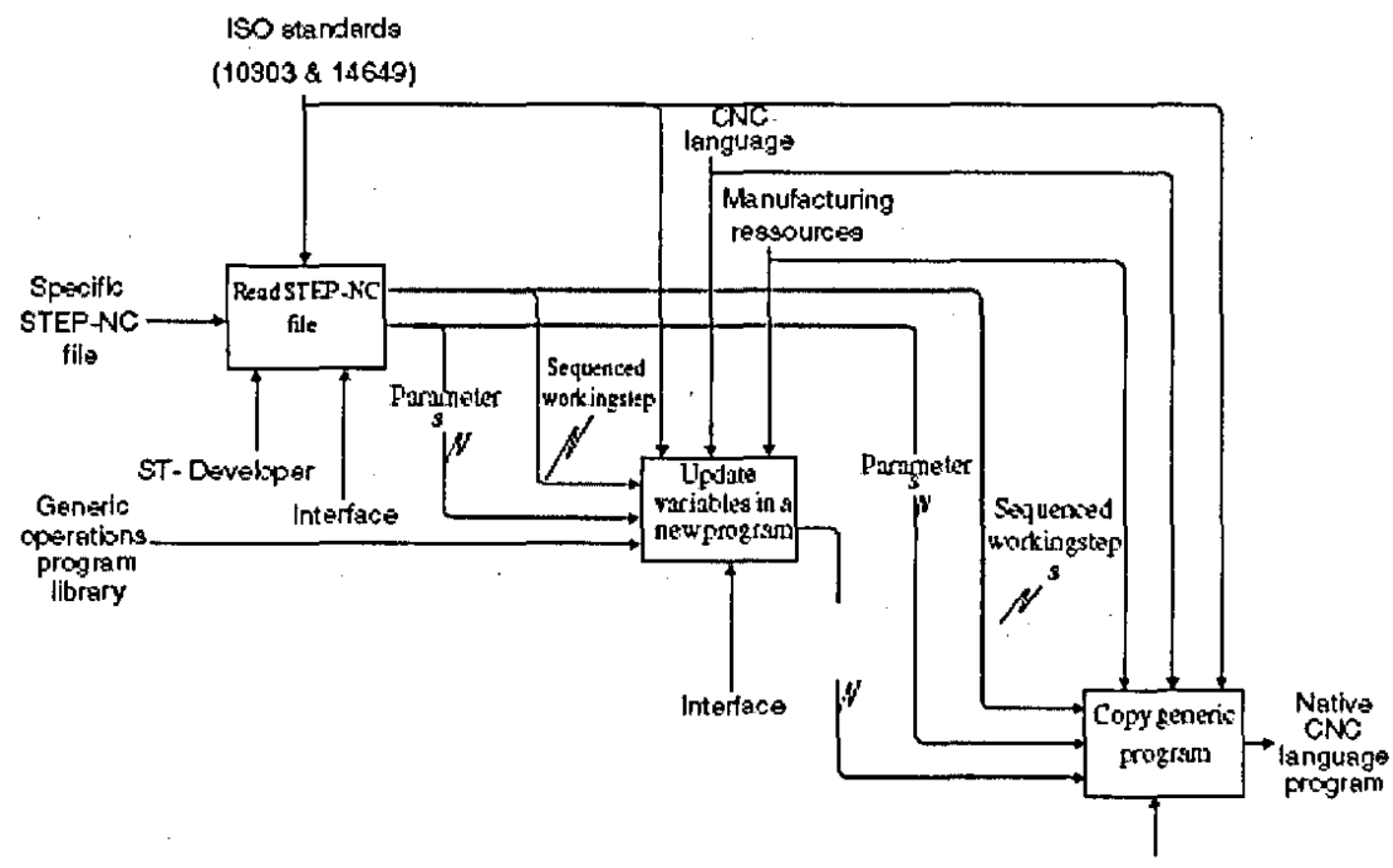

Figure 3.8 IDEF0 diagram of the STEP-compliant CAD/CAPP/CAM/CNC system

(Xu and Wang, 2004)

\subsubsection{G2STEP}

The G2STEP system has been designed based on design and functional considerations developed by researchers from the National Research Laboratory for STEP-NC, POSTECH, South Korea together with a researcher from the EPFL, Switzerland and is based on the ISO 14649 data model, the ARM model and instruction schema on G-code based on the FANUC0 series (Shin et al., 2007). The main proposal for G2STEP is to generate a STEP-NC part program from a G-code program with additional information related to real machining that is easily generated by skilled operators. G2STEP comes with nine functions as summarised below;

i. Pre-processor function: each block of a G-code part program together with other information is interpreted and stored in the pre-defined data structure. These blocks are divided using some clues as to which Gcodes form boundaries to start new machining operations and these blocks are grouped into workingsteps, the basic unit of STEP-NC. 
ii. Machine tool generation function: the tool holder and insert, expressed by the tool maker's catalogue model number, is converted into functional requirements for the model defined in ISO 14649 Part 111 (ISO/FDIS, 2004) and Part 121 (ISO, 2005b).

iii. Technology generation function: technology information such as spindle speed control, feedrate and so on is generated to interpret an S or F-code.

iv. Machine function generation function: machine functions such as coolant on-off, are generated as interpretations of the miscellaneous code (M-code).

v. Machining operation generation function: the machining operation is mainly generated and divided into roughing and finishing. This information needs to use information about tools and technology and hence the machining operation generation function follows the functions which generate these.

vi. Feature recognition function: the feature profile remaining after a workingstep is generated, and the manufacturing feature, defined by STEP-NC, is recognized by a profile and pre-determined machining operation.

vii. Machining strategy generation function: the approach/retract strategy and machining strategy for tool paths are generated.

viii. STEP-NC part program generation function: the enriched information of the workingsteps is stored in succession, one workplan entity includes these workingsteps and finally these are included in one project entity, the first interpreted entity in the STEP-NC data model. This information is instanced in the STEP-NC schema and is printed in a physical file format according to the ISO 10303 Part 21 rule (ISO, 2002).

ix. Part program verification function: the STEP-NC part program is verified and modified through visualization. The machined feature profile is shown and each workingstep can be examined and edited through the workingstep editor. 
G2STEP has been developed for 2-axis CNC Turning using the $\mathrm{C}++$ language and runs on a Windows platform used a geometric modelling kernel and OpenGL for the GUI (Shin et al., 2007). As mentioned, one of the functions for G2STEP begins with a pre-processor to interpret G-code blocks such as instructions, location coordinates, feedrate, spindle speed control and allocated tool number which are stored into groups according to workingstep as shown in figure 3.9. For machining operation generation, G2STEP allows the skilled operator to control the spindle speed and feedrate based on either strong, normal or weak rules that have been determined in each machining operation rule. The subsequent process is feature recognition, based on machining features using Boolean operations with workpiece with machining areas. As stated in ISO 14649, the machining strategy for turning operations refers to bidirectional, unidirectional, contour or grooving strategies. Again the machining strategy is determined from strong, normal or weak rules.

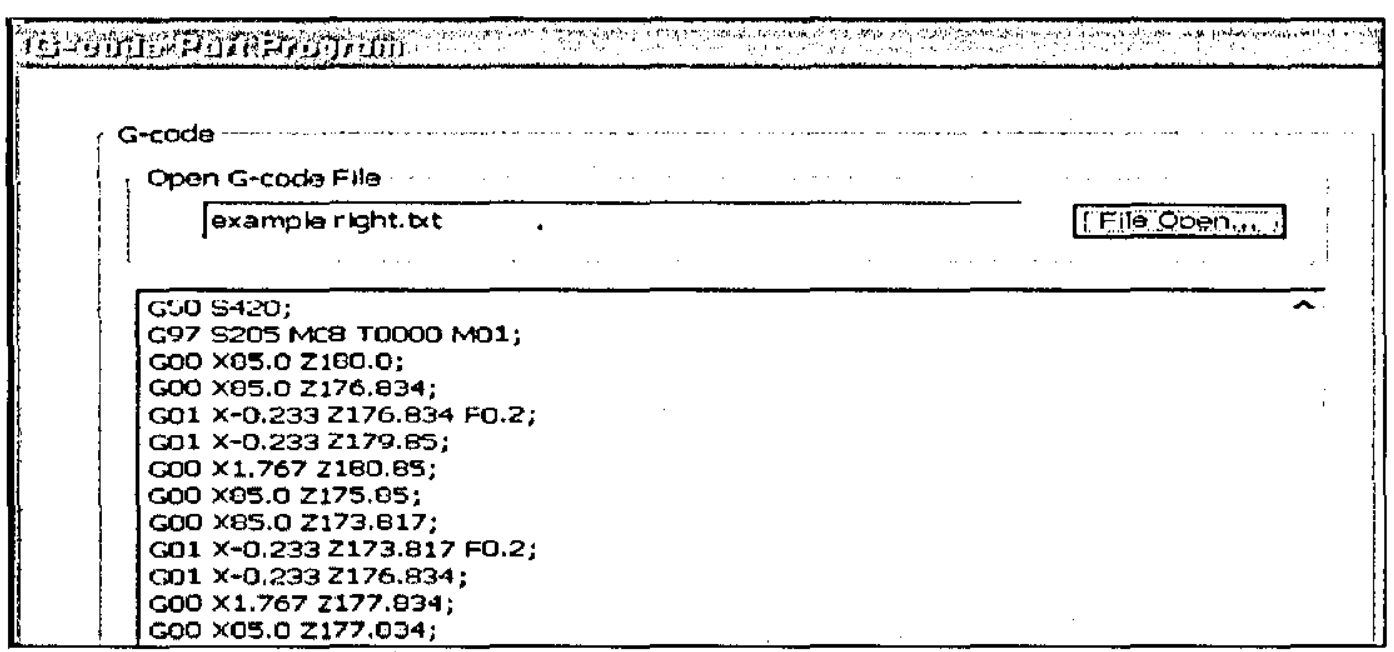

Figure 3.9 G-code load to G2STEP system (Shin et al., 2007)

The commercial controller to interpret STEP-NC part programs has not been launched yet and this prototype provides a basic simulation only. G2STEP has been verified through Virtual NC software from Delmia. G2STEP is at the prototype 
stage and future work is needed to add milling applications, multi-axis and complex machining (Shin et al., 2007).

\subsection{Review Discussion}

There is no doubt, that so far none of the proposed systems are fully capable of machining turn/mill components. Work to date has focused on the separate parts of ISO 14649 using Part 11 for milling operations including drilling and Part 12 for turning. No significant work has been done on combining the two parts for turn/mill components. However, the author and some researchers (Heusinger et al., 2006) believe that this industrial requirement could be achieved through research and development involving collaboration by researchers, users, manufacturers, academia and the ISO committee. If developers look from the business perspective, and academia focuses on theoretical aspects the objective of combination turning and milling machining compliance with the new standard (STEP-NC) can be realised.

If we focus on turning operations, only three proposed systems are available, STEPTurn, TurnSTEP and G2STEP. But, if we scope for e-manufacturing, STEPTurn leads in this aspect due to the capability of internet file transfer. TurnSTEP clearly defines the number of set-ups as either one set-up or two set-ups dependent on the independent machine format (Suh et al., 2006). TurnSTEP has some weaknesses such as threads cannot be automatically generated but need to be defined and the process plan graph edited by the user manually. The output of this system can be in text and XML file formats (Suh et al., 2006). As reported TurnSTEP is at a prototype stage and the implementation of another part, which is intelligent and autonomous is still under development.

In terms of implementation of bi-directional information flow, none of the systems show how it would work and do not make it clear how the functionality is supported in prototype systems. So far the test components used contain only simple turning operations with $\mathrm{z}$ and $\mathrm{x}$ axes and do not cover multi-axis machining. The author strongly agrees with the suggestion by Heusinger and Rosso-Jr, for the 
STEP-NC compliant information structure to support the milling capability of the NC turning centre to meet industrial needs mapped by ISO 14649 Part 11 and 12 (milling and turning) (Heusinger et al., 2006; Rosso-Jr et al., 2004).

The author has noticed that all the proposed systems use a feature recognition approach and feature based techniques to allow the user to edit the part program. Xu has stressed that the commercial software, namely ST-Plan, can create STEP AP 224 machining features from CAD files (AP 203 or AP 214) (Xu and Newman, 2006). All the proposed systems comply with ISO 14649 and this is the first stage to develop the universal manufacturing platform for $\mathrm{CNC}$ machining as proposed by (Choi et al., 2006; Newman et al., 2007).

\subsection{Summary}

This chapter provides a literature review of the STEP-NC compliant research around the world focused on STEP-Compliant NC Research Projects; the intelligent manufacturing system (IMS) and the intelligent manufacture for STEPNC compliant machining and inspection as an overall view based on STEP-NC. The IMS and intelligent manufacture for STEP-NC compliant machining and inspection project was successful developed and involved both industries and academia around the world. The second part of the chapter concentrated on the systems or prototypes which have been developed by other researchers. STEPCompliant systems for the manufacturing environment have been reviewed focused on turning operations such as the Shop-Floor Programming System (SFPS), STEPTurn, TurnSTEP, G-code free for lathe and G2STEP. All the systems have advantages and disadvantages and the opinions are based on published information rather then first hand experience. The next chapter discusses the design of the STEP compliant system framework. 


\section{Chapter 4 - Design of the STEP-NC Compliant System Framework}

\subsection{Introduction}

In order to reach the second objective of this research (section 1.4), it is necessary to outline the design and framework of the proposed system based on information from the literature review and the model developed by the author. This chapter discusses the design and framework of the STEP Compliant CAPP/CAM system for turning operations, by referring to ISO 14649 Part 1, Part 10, Part 12 and Part 121. The ISO document specifies the process data which is generally needed for NC programming within all machining technologies. Part 121 refers specifically to process data for turning including tools for turning machines. This framework covers questions such as i) where to start, ii) what to machine, iii) how to make things happen and iv) how to machine. The chapter starts with the design and tools for SCSTO. The chapter then discusses the fundamentals of workpiece and turning manufacturing features, followed by machining workingsteps for turning, and machining operations for turning. The chapter concludes with a discussion of the framework model for the proposed system.

\subsection{Fundamentals of Machining Process Planning}

The fundamentals of planning a machining process in a numerically controlled environment lie with the control and quality of operation planning and that planning time represents 50 to 80 percent of the actual machining time for 
single parts or small batches (Ahlquist, 2002). It becomes more critical for complex situations and new manufacturing technologies tend to extend the time further. Process planning has been defined by (Alting and Zhang, 1989) as a function within the manufacturing environment which deals with the selection of manufacturing processes and parameters to be used to create the final product (Alting and Zhang, 1989). Investigations by Younis showed that an efficient CAPP system could result in reduction of the manufacturing costs by up to $30 \%$ and would also reduce the manufacturing cycle and the total engineering time by up to $50 \%$ (Younis and Wahab, 1997). Hence, the focus has been on process planning as the task of the determination of manufacturing processes, which for instance can determine whether or not a product should be manufactured through turning operations.

\subsection{Design of a STEP Compliant System for Turning Operations (SCSTO)}

This section proposes a system framework for a STEP Compliant System for Turning Operations (SCSTO) which considers both informational and functional perspectives of the system. The information perspective will be discussed in Chapter 5. From a functional perspective the proposed system has been designed to be a semi-automatic process planning system, meaning that it does not automatically generate manufacturing information directly from the CAD model. It is aimed at the creation of feature-based process plans for manufacturing processes such as turning operations. The proposed system is for turning operations and is based on a STEP compliant environment. It consists of several elements that define turning features and generate STEP code compliant with ISO 10303 Part 21 (ISO, 2002). The system is based on feature-based design and begins with the selection of the workpiece followed by the choice of turning manufacturing features and finally the choice of the tools. The output of the system is a physical file complying with Part 21 (ISO, 2002).

The aim of this work is to address the process planning and machining of rotational components and to propose a STEP Compliant NC structure for generation of ISO 14649 code which can be used for turning component 
manufacture. Interoperability within this context is a significant objective. Interoperability is defined as the ability to integrate STEP-NC compliant information in the product life cycle including CAD, CAPP, CAM and CNC, combined with feasible information structures to represent various configurations of turning machining centres. The overall framework is illustrated in figure 4.1 and is based on the Java programming language. The prototype has been developed using JBuilder 2005 (Landy et al., 2003) to provide a suite of integrated development tools related to STEP standards. This concept has been used to generate java classes from the EXPRESS schema and to handle the STEP Part 21 physical file format. Brief details of the system will be presented in Chapter 6 .

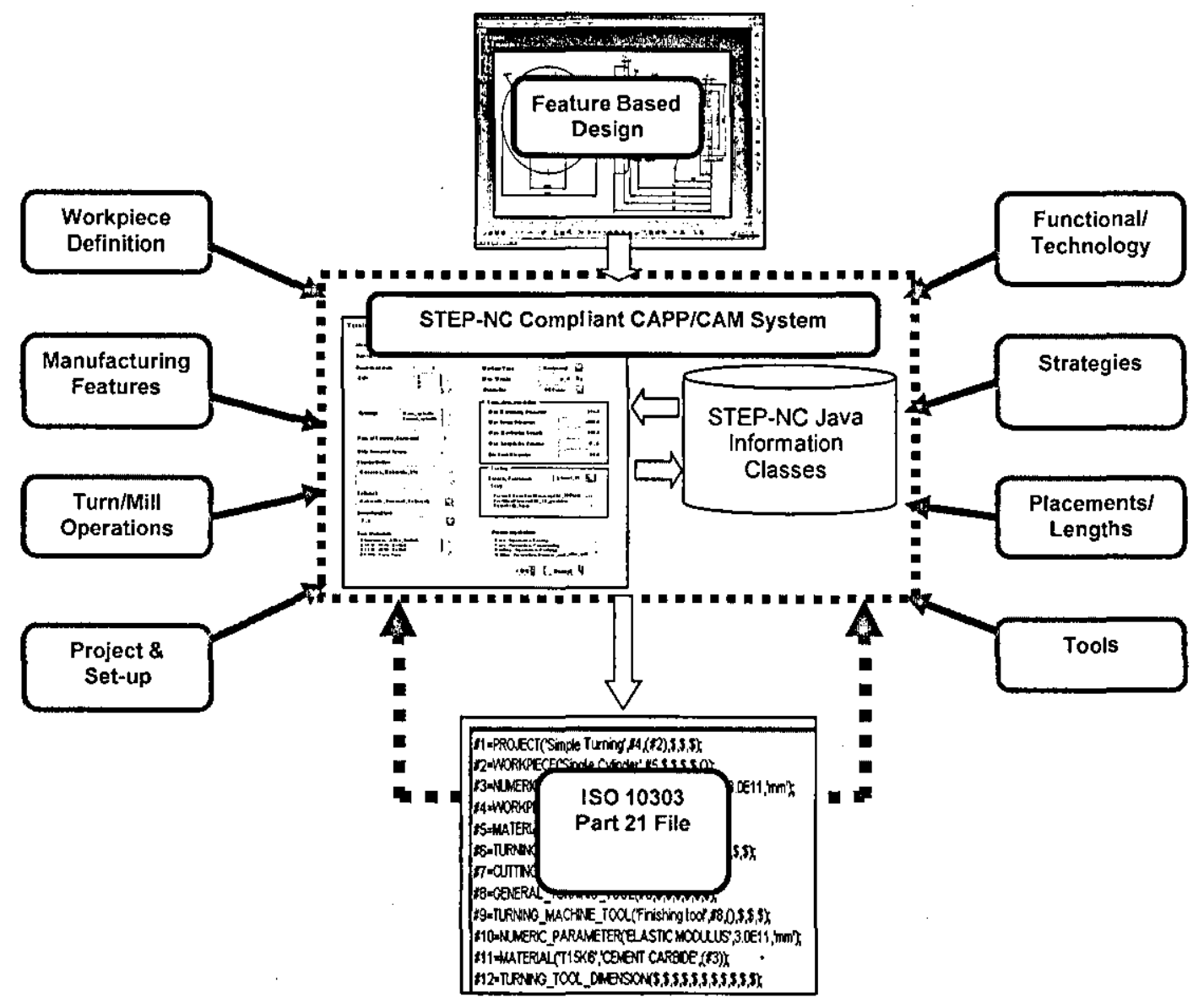

Figure 4.1 Proposed System 
The data model for manufacturing of turned components was based on the ISO 14649 standard. Part 10 is the backbone of the standard covering the common data structure. In the standard, the part is defined as a workpiece while the task is defined as a workplan consisting of a series of machining_workingsteps to carry out the machining_operation on a manufacturing_feature. In turning operations it becomes a workplan with a series of turning_workingsteps to carry out the turning_operation on a turning_feature. The turning_operation itself is supported by turning_technology, turning_machine_function and turning_strategy as shown in figure 4.2. An overview of the ISO 14649 data model structure is shown in figure 4.3. for the proposed system. This diagram is a summarised by the excerpt EXPRESS_G diagram (figure 4.3) from ISO 14649 Parts10, 11 and 12 (ISO, 2004a; ISO, 2004b; ISO, 2005a).

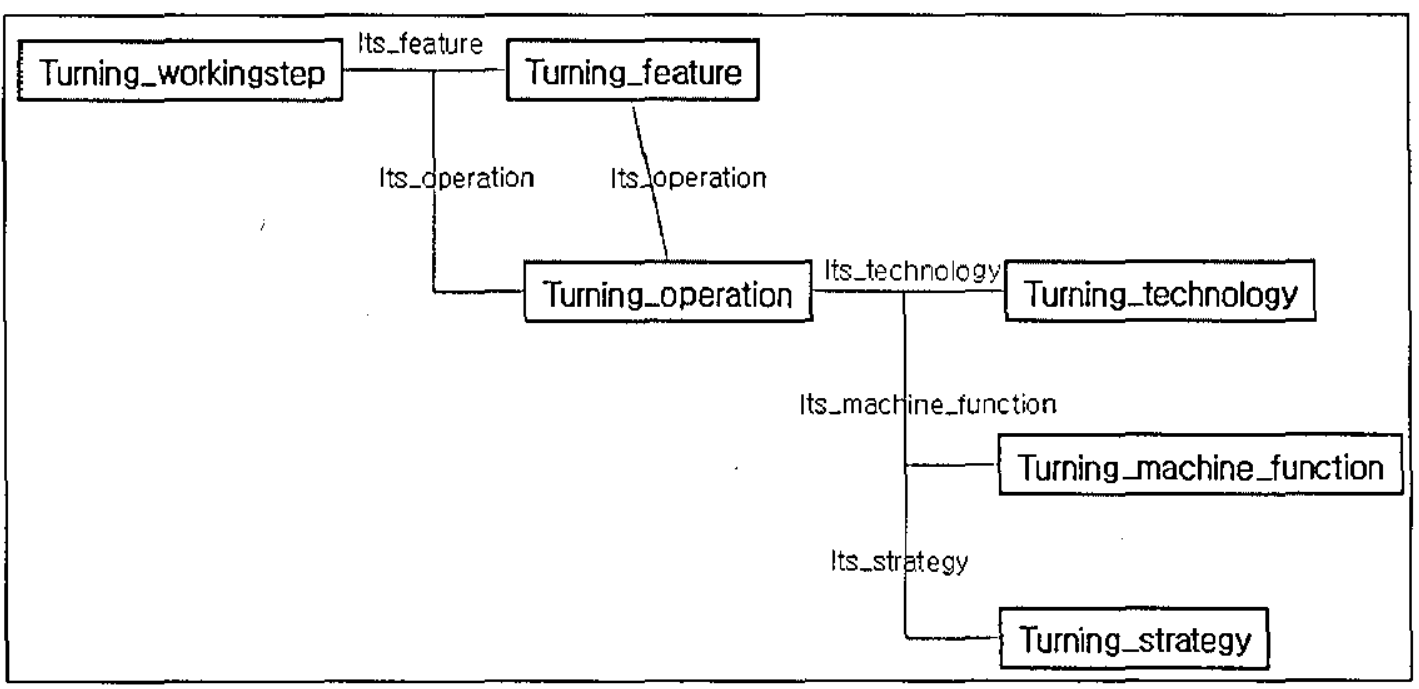

Figure 4.2 Overview of the turning operation based on STEP-NC adopted from (ISO, 2005a) 
Chapter 4

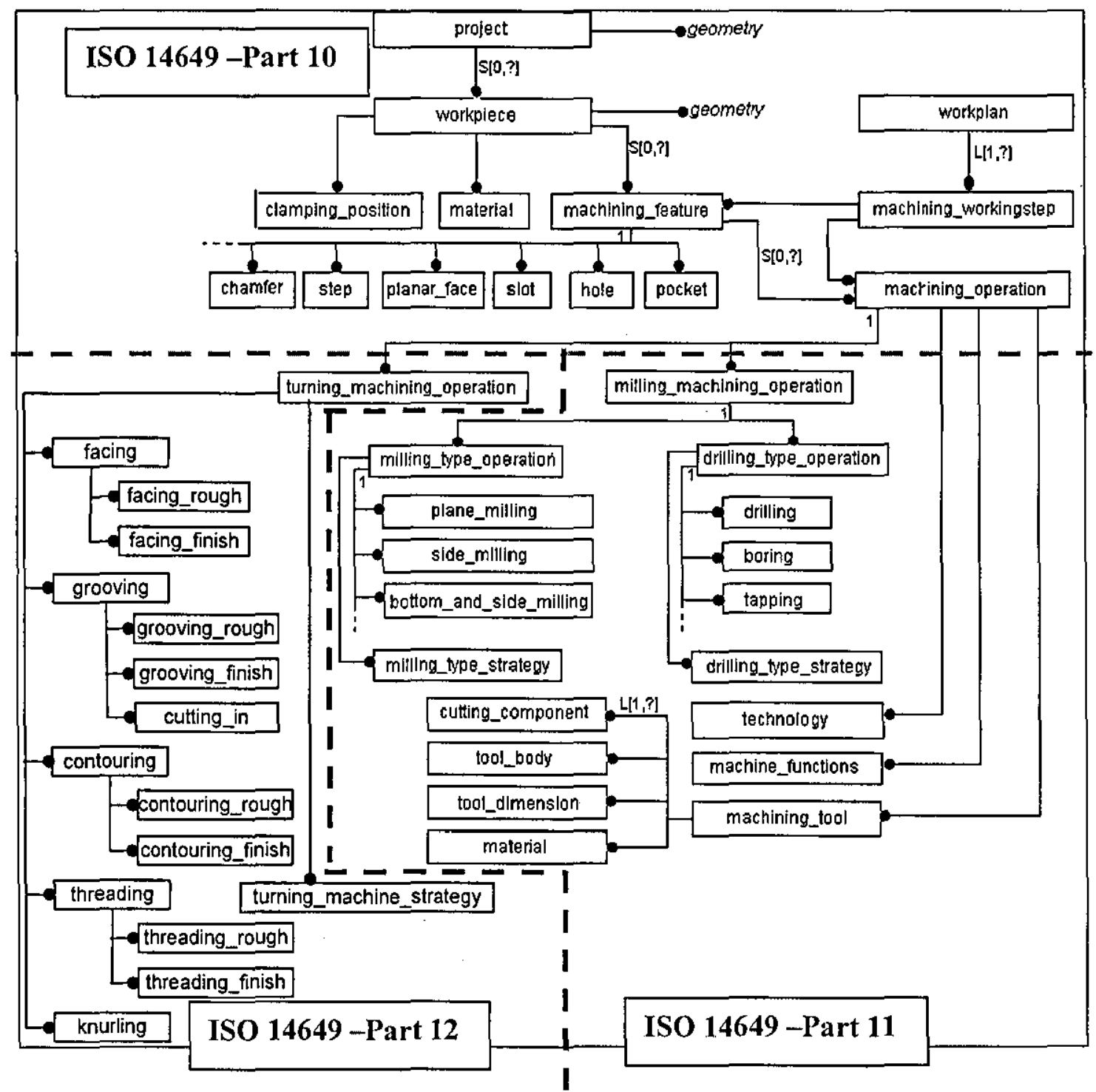

Figure 4.3 Overview of STEP-NC data structure (ISO, 2003b; ISO, 2004a; ISO, 2004b; ISO, 2005a)

Both product and manufacturing information models are considered and described in Chapter 5. The major activities of the proposed system include the translation of geometrical features, information database tools, the planning of machining operations and the generation of the STEP-NC process plan (ISO 10303 Part 21 file). The proposed system also considers the CNC machine capability which in this research is limited to a maximum of six (6) axes. Figure 4.4 shows the 
interoperable manufacturing environment for turning operations. The output from the system is a process plan with corresponding CAx systems or CNC machines.

\subsection{Interoperable Manufacturing Environment}

The proposed system framework supports data interoperability between the various CAx systems in the manufacturing chain. Figure 4.4 illustrates how the proposed system operates in an interoperable manufacturing environment.

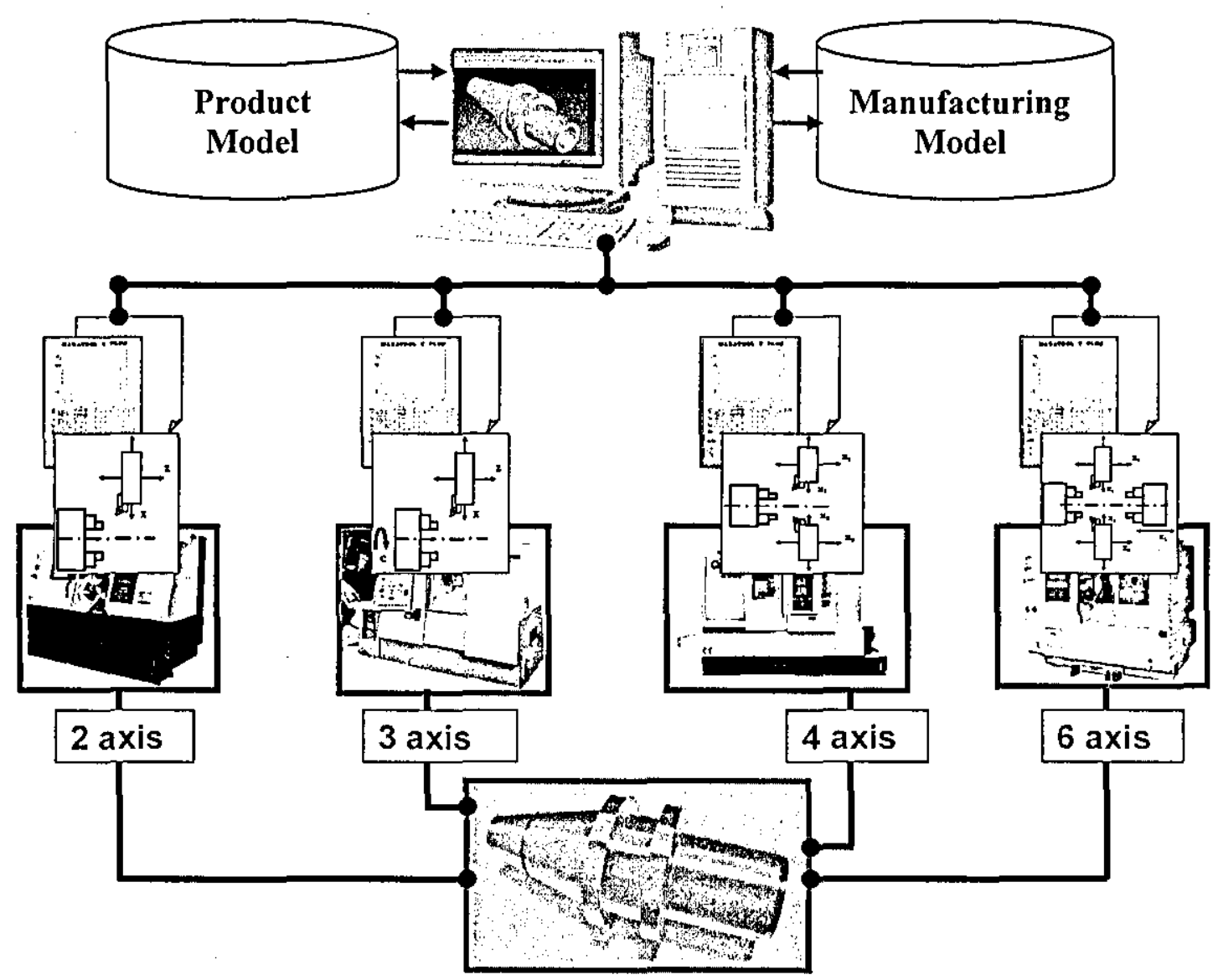

Figure 4.4 Interoperable Manufacturing Environment for Turning Operation 


\subsection{Application Tools for SCSTO}

The implementation of SCSTO is based on the application of STEP-NC. The realisation of the system together with the supporting information models is presented in Chapter 5. The system has been designed through the use of the UML class diagrams and JDataStore (Borland, 1997) together with the java programming language. Product, process and resource models have been used based on the STEP method to represent products, processes and the information to support communication. Well-established information models have been used to model the information and the user interactions with the system. The details of the product, process and information models will be discussed in chapter 5 .

\subsubsection{STEP}

STEP is an unofficial name; the actual designation of the STEP standard is ISO10303 Industrial automation systems - Product data representation and exchange. (for details refer to Chapter 2 , section 2.4).

\subsubsection{UML}

UML has been used to represent the various objects in the turning manufacturing environment and the relationships between these objects. UML is an industry standard and object oriented modelling language that provides a reliable architecture for specification, visualisation and documentation of information models (Quatrany, 1998). UML is used as an information model and consists of a number of UML class diagrams. It has enabled the design along with the structure of the product and process knowledge to be explicitly illustrated and easily implemented in the database management system (DBMS). The JDataStore DBMS (Borland, 1997) is employed in the system to store, modify and extract data from the database and allows it to be shared among multiple applications (for details refer to Chapter 5, section 5.7). 


\subsection{Major Activities of system}

The major activities of the proposed system include the translation of the geometrical features, planning of machining operations and generation of the STEPNC process plan. These turning process planning activities are outlined below;

i) Translation of turning features (section 4.7)

ii) Turning feature library (section 5.8)

iii) Specification of base part (section 4.8)

iv) Machining operation planning (section 4.9)

v) Tool Database (section 4.10)

vi) Process plan generation (section 4.11)

\subsection{Translation of Turning Features}

In the proposed system, a feature extraction approach is favoured rather than feature based design due to the ability to extract features from a library based on ISO 14649 Part12 turning features. Machining operations for turning mainly focus on ISO 14649 part 10 and 12. Part 10 describes general machining operations and part 12 defines turning machining operations and machining strategies (ISO, 2004a; ISO, 2005a). Suh illustrated his view of both manufacturing features (Part 10) and turning features including outer round, revolved features, knurl and cut in (Part 12) process data for turning (Suh and Cheon, 2002), as shown in figure 4.5. The basic elements of the turning operation model that have been developed so far include technology and strategy, machine functions and tools (Heusinger, 2003). 


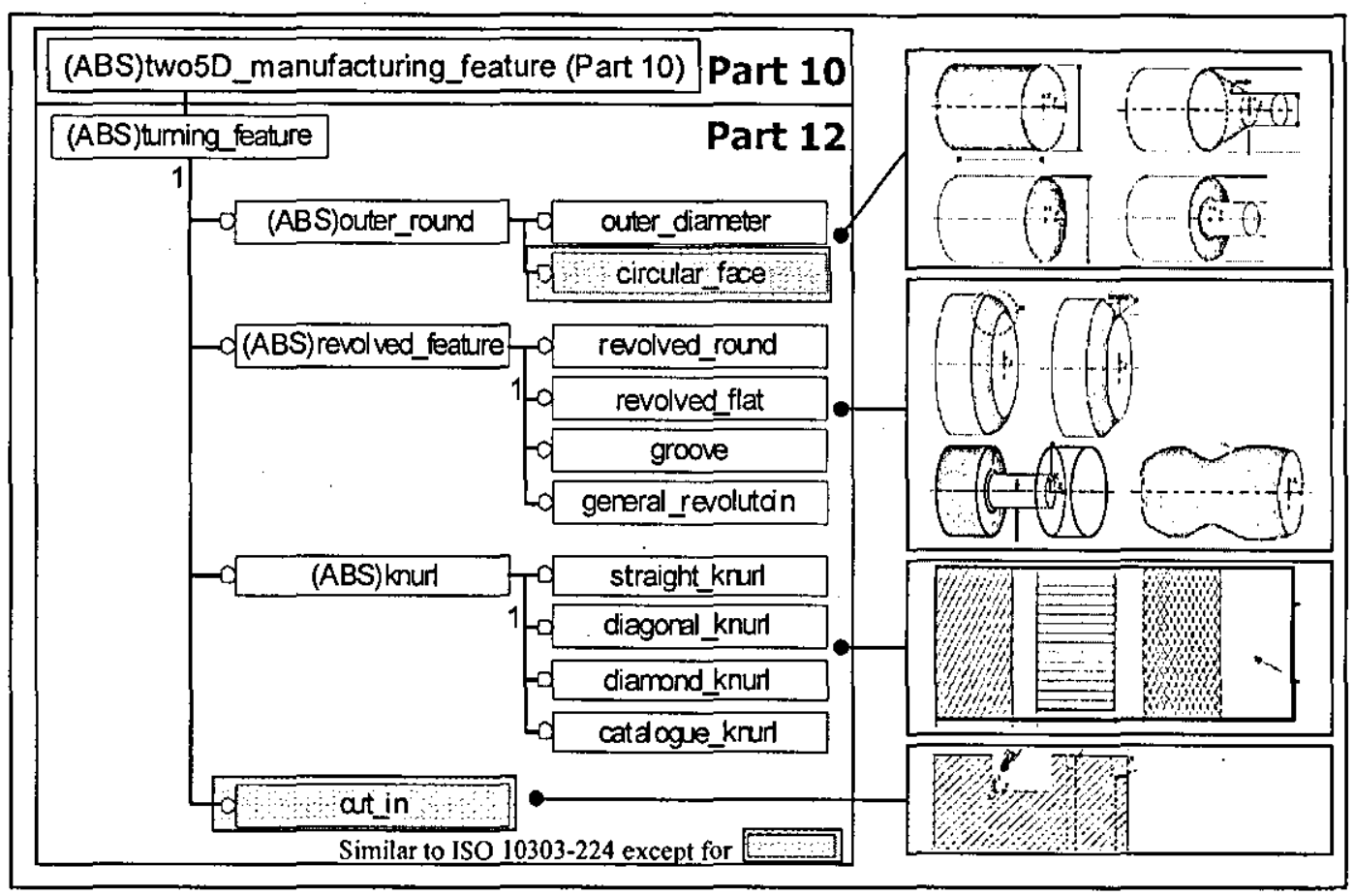

Figure 4.5 Manufacturing Features and Turning Features (Suh, 2002)

Referring to ISO 14649-12 (ISO, 2005a), the following features are currently included: outer_round (outer_diameter, outer_diameter_to_shoulder), revolved_feature (revolved_flat, revolved_round, groove, general_revolution), knurl (straight_knurl, diagonal_knurl, diamond_knurl, tool_knurl). Features supported by SCSTO are restricted to those based on STEP AP224 which was developed for mechanical product definition for computerised process planning using form features (ISO10303-224). Figure 4.6 shows the structure of the turning_feature entity within the manufacturing_feature class (ISO, 2004a; ISO, 2005a).

A manufacturing_feature identifies the types of features necessary to manufacture a component. Each manufacturing feature is either a transitionffeature, two5D_manufacturing_feature or region. Each transition feature type, is either an edge_round, fillet or a chamfer. Each region class has another three subclasses i) region_projection, ii) region_surface_list and 
iii) topological_region. However in this research the focus is on two5D_manufacturing_features and more specifically on turning_features.

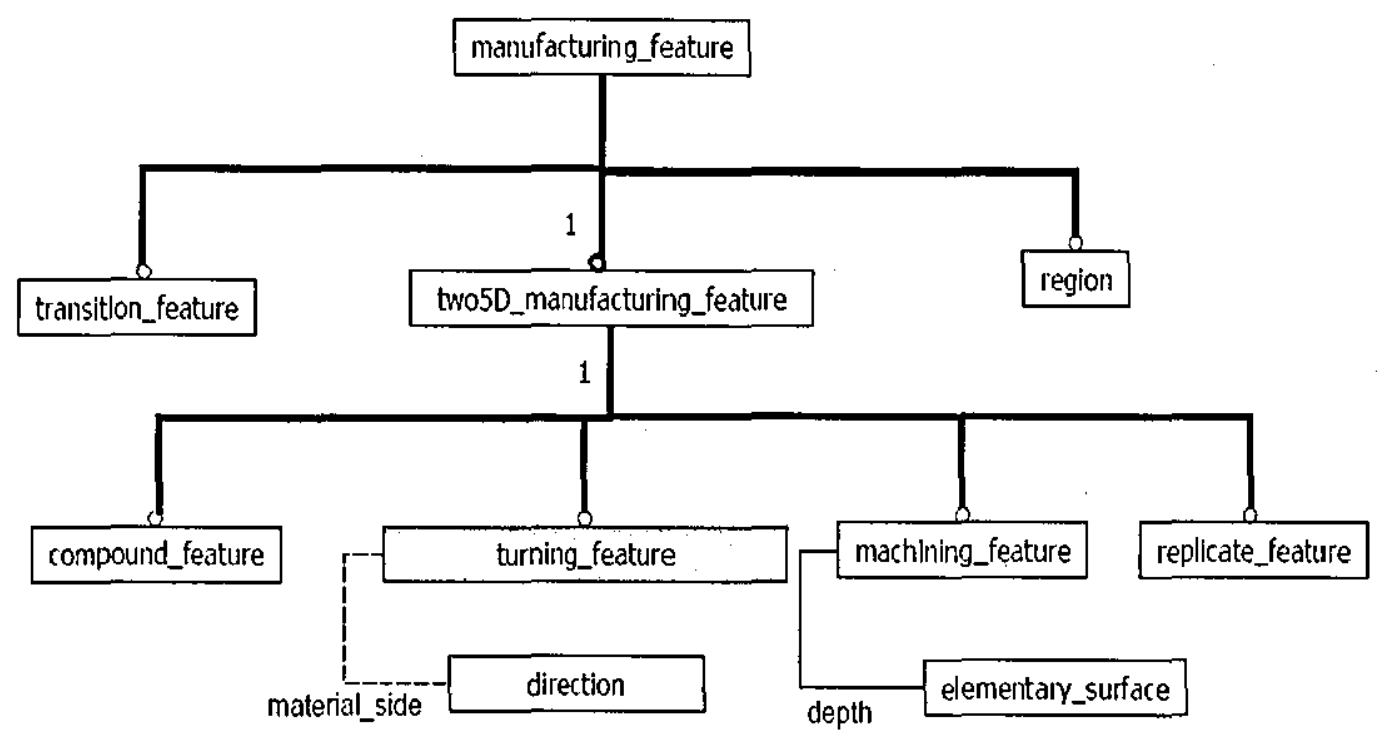

Figure 4.6 Location of turning feature adopted from ISO 14649 Part 10

$$
\text { (ISO, 2004a) }
$$

Figures 4.7 and 4.8 show the UML format and EXPRESS-G representation of ISO 14649-12: Process Data for Turning. This standard represents the turning_feature within the abstract subtype (ABS) two5D_manufacturing_feature and also within the outer_round, revolved_feature and knurl subtypes. An outer_round is a type of turning_feature that is an outline shape that is swept through a complete revolution about an axis, either outer_diameter or an outer_diameter_to_shoulder. (See section 4.7 for detail turning features graphic).

A revolvedffeature is a type of turning feature that is the sweeping of a planar shape one complete revolution about an axis. Each revolved feature is one of the following; revolved_flat, revolved_round, groove or a general_revolution. 
Chapter 4

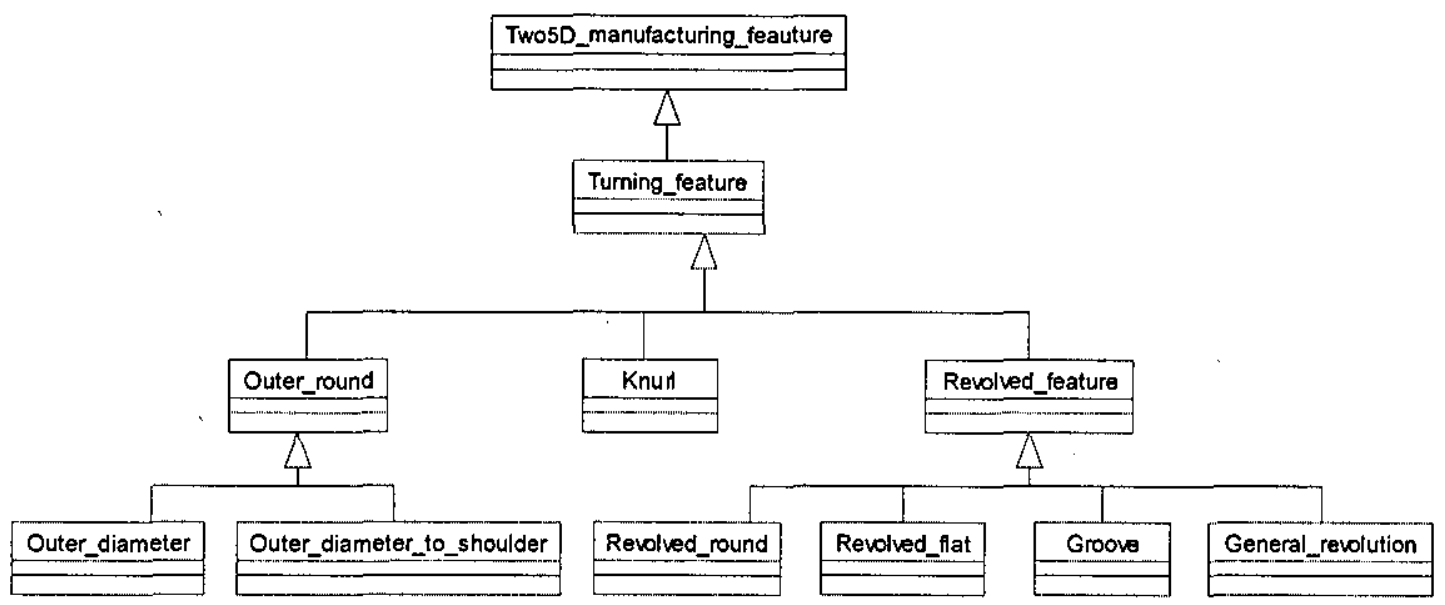

Figure 4.7 The turning feature and its subtypes described in UML format.

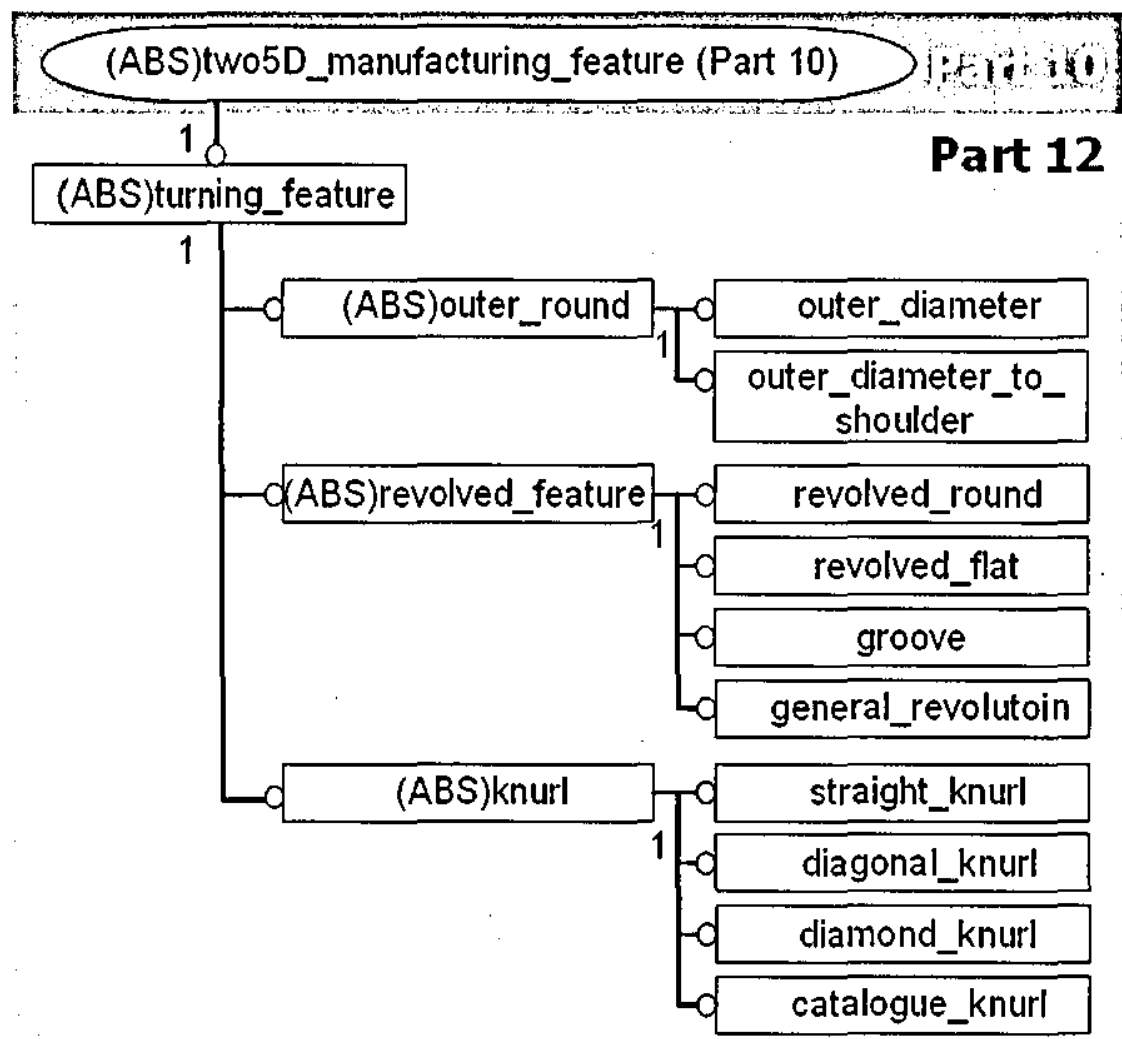

Figure 4.8 Manufacturing Features for Turning 
Figure 4.9 shows the combined structure for ISO 14649 Parts 10 and 12 illustrates represent turning features in EXPRESS-G diagram.

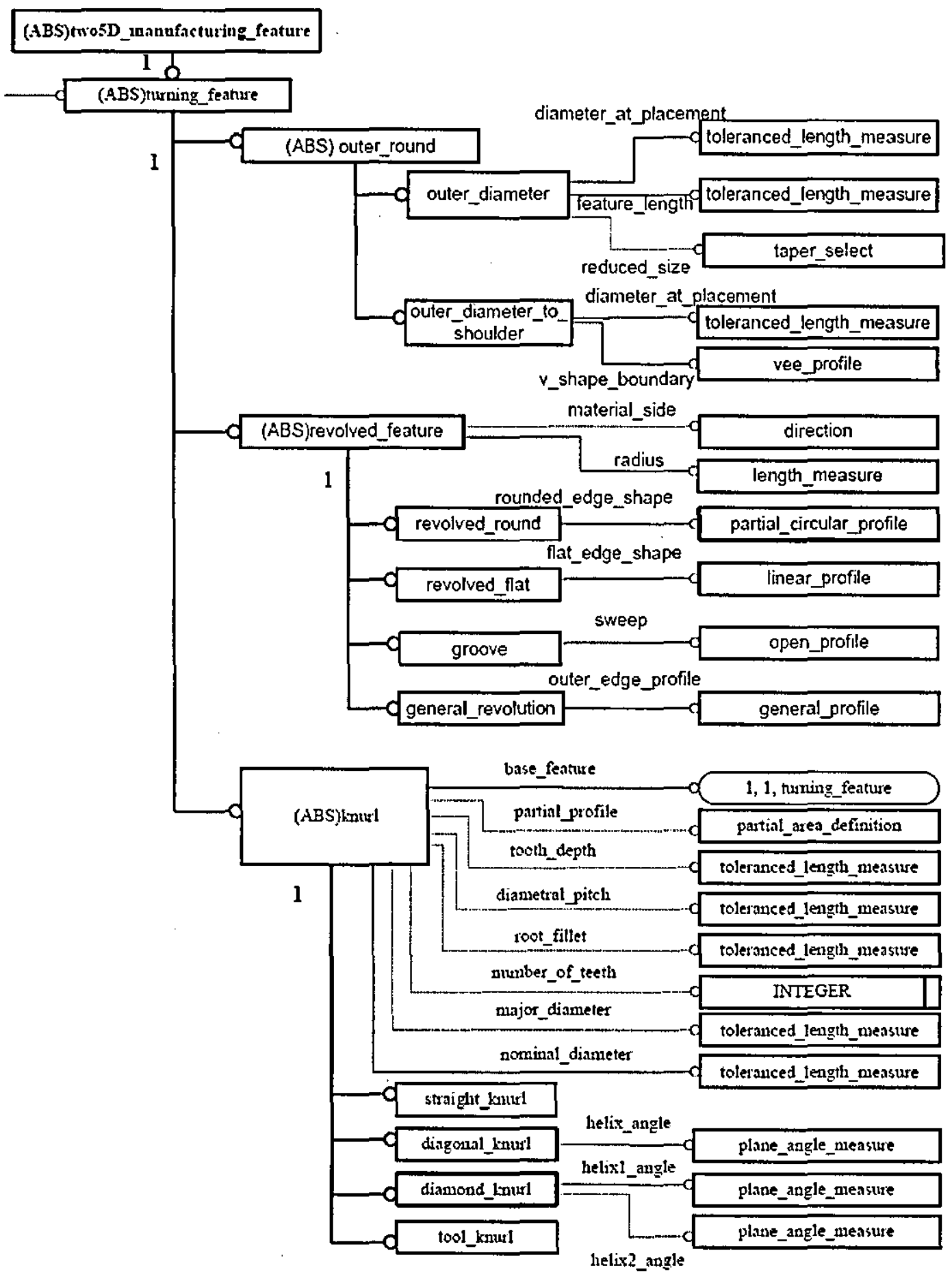

Figure 4.9 EXPRESS-G representation of ISO 14649-12: Process Data for Turning. (ISO, 2005a) 


\subsubsection{Outer_round}

An outer_round is a type of turning_feature that is an outline or significant shape that is swept through a complete revolution about an axis. Each outer_round is either an outer_diameter or an outer_diameter_to_shoulder. The axis of revolution should be the same as the $z$-axis of the feature. These words can be translated into the EXPRESS language as follows;

\section{ENTITY outer_round}

ABSTRACT SUPERTYPE OF (ONEOF (outer diameter, outer diameter to shoulder))

SUBT̄YYE OF (turning feature);

END_ENTITY;--Outer_round

Outer_diameter The outer_diameter is a subtype of outer_round that is the sweeping of an outline specified by a line segment by one complete revolution about an axis. The line is finite in length and coplanar with the axis. The outer_diameter (figure 4.10) may have a constant diameter around the axis of rotation that is straight_outer_diameter, or it may be tapered that is tapered_outer_diameter. Diameter, feature_length and reduced_size are the parameters necessary to completely define the outer_diameter geometry. In the case of the definition of a cylinder the diameter_at_placement and the feature_length are sufficient. A cone describes a continual transition from one diameter to another diameter across a certain feature_length and for its definition the additional attribute reduced_size is used. These words can be translated into the java language as follows; 
package iso14649;

// Imports

import java.util.ArrayList;

public class outer_round extends turning_feature \{

// Constructors

public outer_round 0\{\}

public outer_round(String_its_id, workpiece_its_workpiece,

machining_operation[] _its_operations, axis__placement_3d_feature_placement)

\{\}

\section{// Methods}

public ArrayList get_prop_names() \{return null;\}

public void set_attributes_from_part21_line(String part21line, ArrayList A) \{\}

public ArrayList get_attributes() \{ return null; \}

public ArrayList get_output() \{return null; $\}$

\}

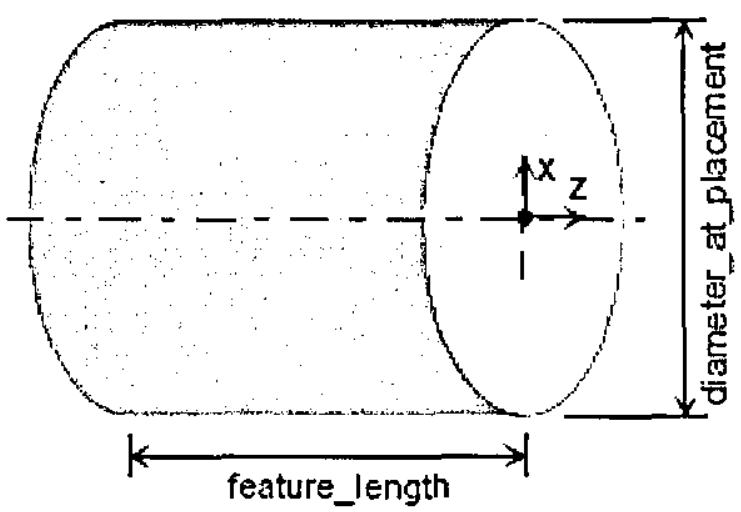

Figure 4.10 Outer_diameter (ISO, 2005a)

\section{Outer diameter to shoulder}

An outer_diameter_to_shoulder as shown in figure 4.11 is a type of outer_round that is a sweeping of a shape one complete revolution about an axis. The shape is specified by two lines that are connected at a point and extend infinitely. The enclosed angle must be smaller than $180^{\circ}$. 


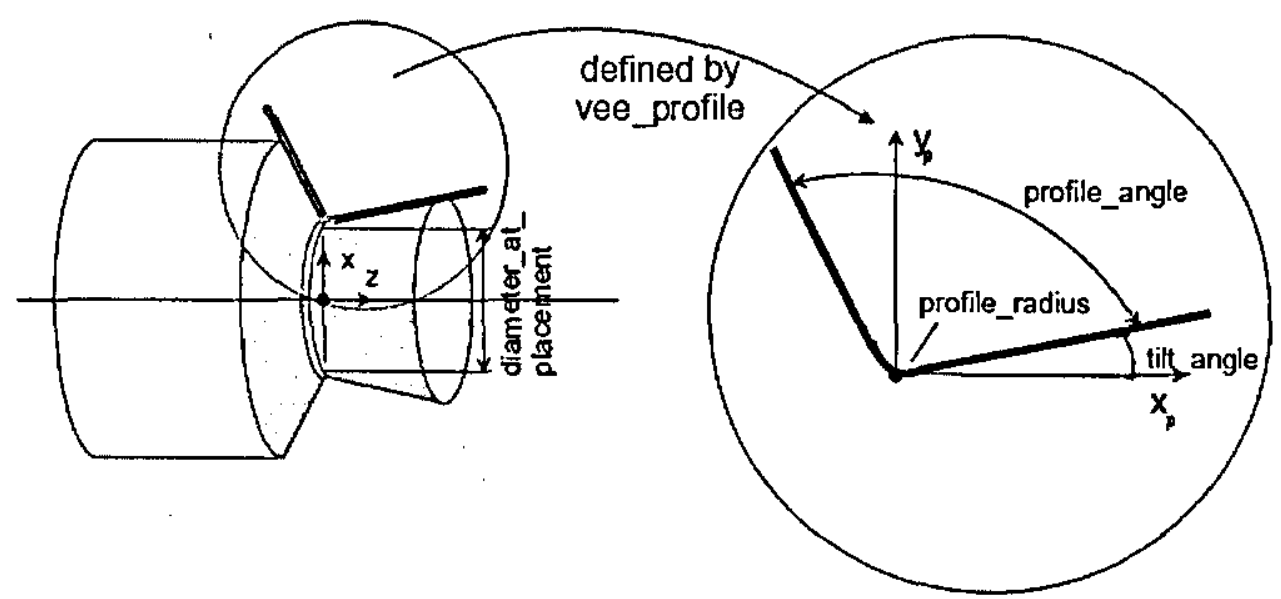

Figure 4.11 Outer_diameter_to_shoulder (ISO, 2005a).

\subsubsection{Revolved_feature}

\section{Revolved flat}

The revolved flat as shown in figure 4.12 is a type of revolved_feature that is the sweeping of a straight line about an axis.

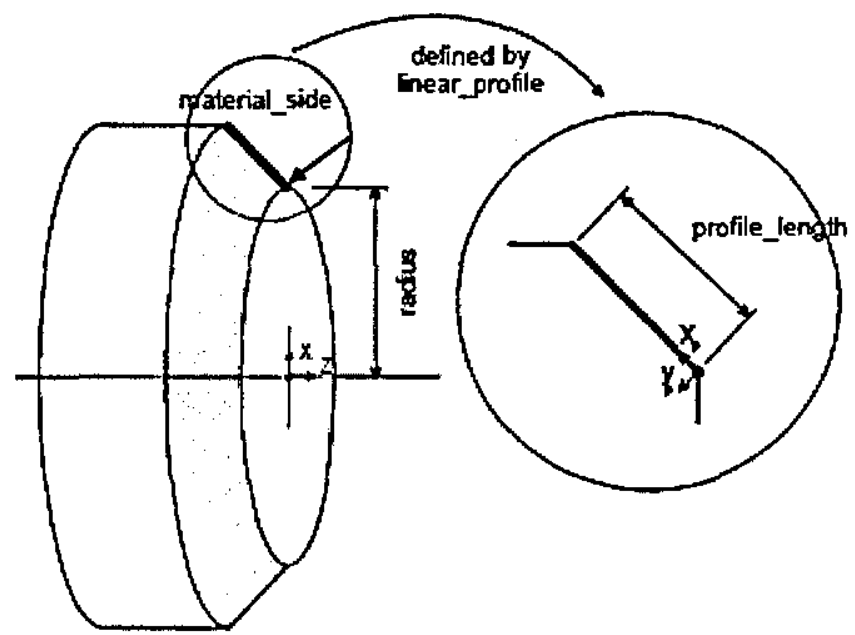

Figure 4.12 Revolved_flat (ISO, 2005a)

Revolved round

The revolved_round as shown in figure 4.13 is a subtype of revolved feature that is the sweeping of an arc about an axis. 
Chapter 4

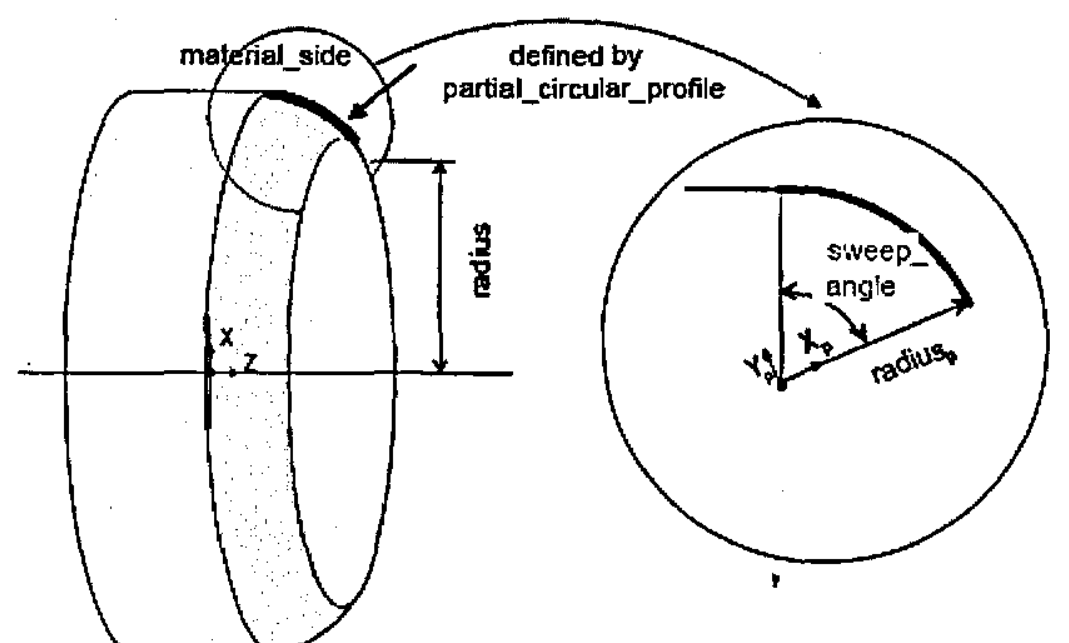

Figure 4.13 Revolved_round (ISO, 2005a)

\section{Groove}

The groove as shown in figure 4.14 is a type of revolved_feature that is a narrow channel or depression that is swept through one complete revolution about an axis. The face shape that has the groove applied to it is determined by the profile orientation.

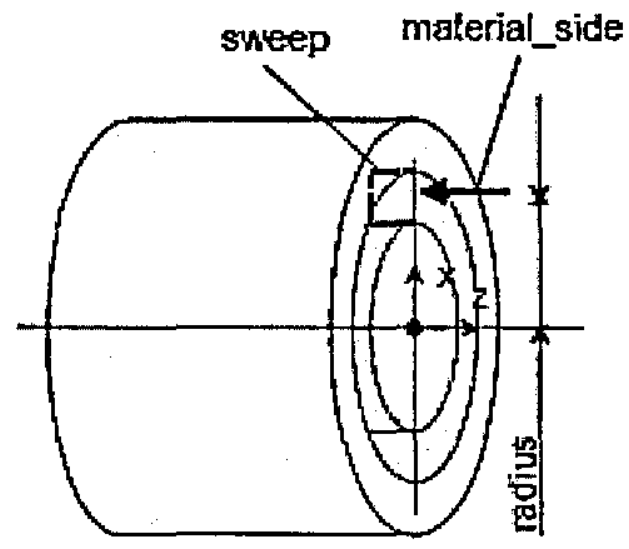

Figure 4.14 Groove (ISO, 2005a)

General revolution

The general_revolution as shown in figure 4.15 is a subtype of revolved_feature that is an arbitrary planar shape swept one complete revolution about the z-axis. The arbitrary planar shape is finite in length, coplanar with the axis of revolution 
and must not intersect the axis of revolution. The general_revolution may be either an outer shape of a part or a removal volume, depending on the material direction.

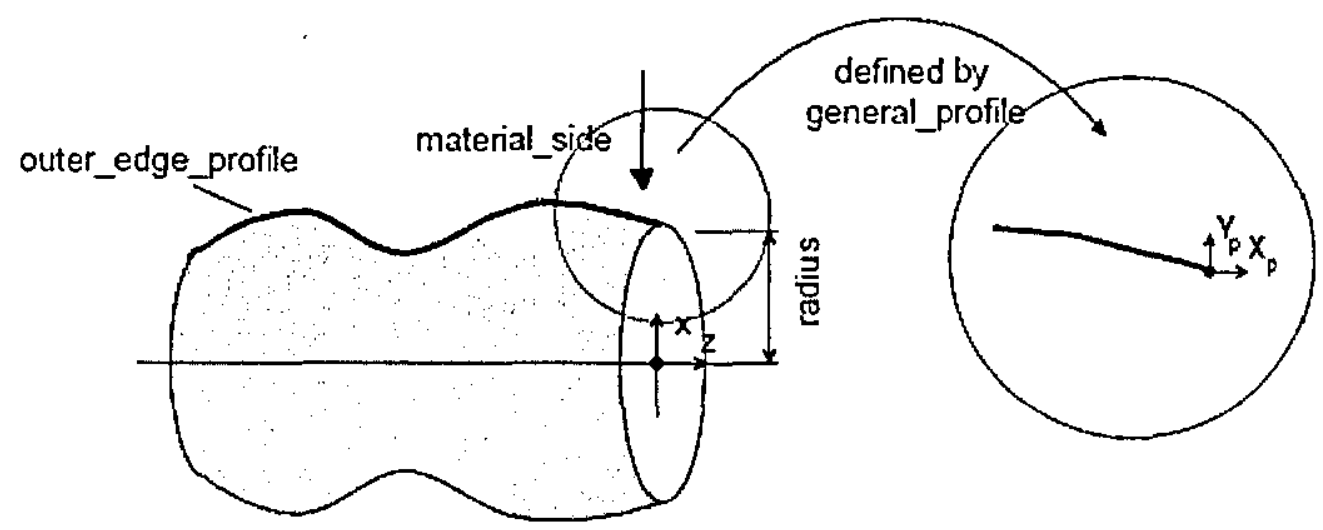

Figure 4.15 General_revolution (ISO, 2005a)

\subsubsection{Knurl}

\section{Straight knurl}

A straight_knurl as shown in figure 4.16 is a type of knurl in which the knurl scoring is parallel to the axis of the scored surface (z-axis). In figure 4.16, x, $\mathrm{z}$ and $\mathrm{x}_{\mathrm{k}}, \mathrm{z}_{\mathrm{k}}$ indicate the placement of the base feature and knurl, respectively.

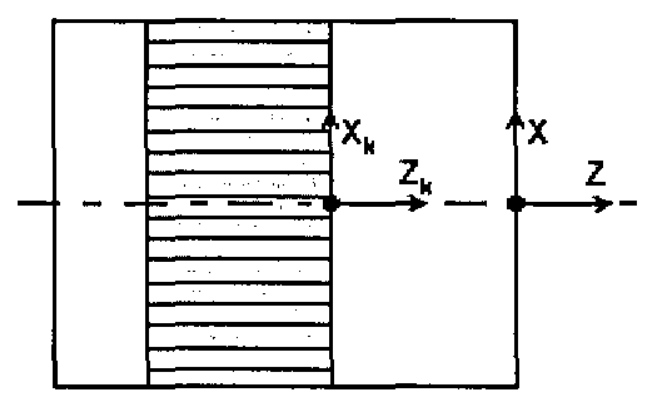

Figure 4.16 straight_knurl (ISO, 2005a)

Diagonal_knurl

A diagonal knurl as shown in figure 4.17 is a type of knurl which makes it possible to define helical cuts at an angle about the axis of a surface. 


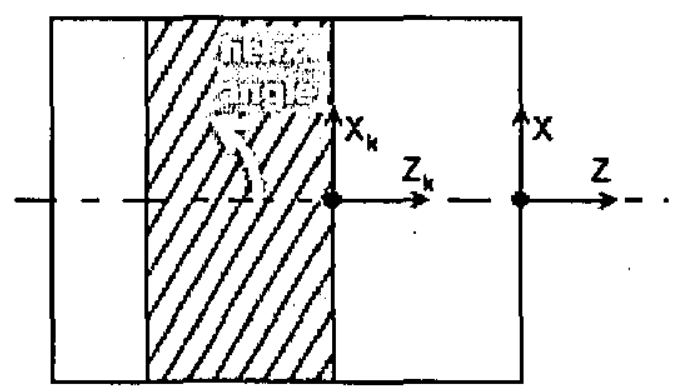

Figure 4.17 Diagonal_knurl (ISO, 2005a)

Diamond_knurl

A diamond_knurl as shown in figure 4.18 is a special knurl with two helical cuts on its surface. The angles of the two helixes are independent of each other.

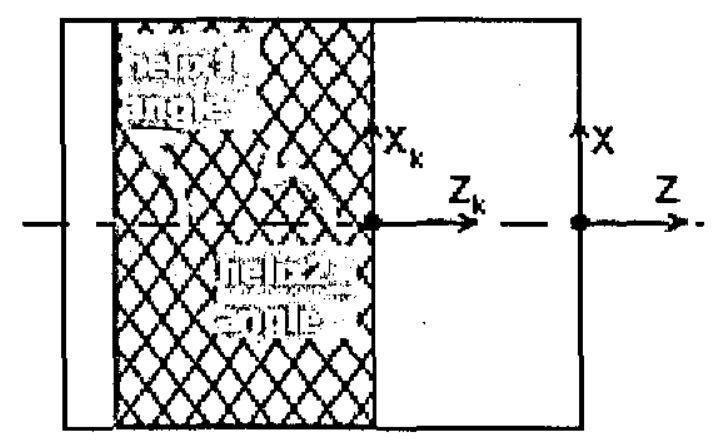

Figure 4.18 Diamond_knurl (ISO, 2005a)

Tool_knurl

A tool_knurl is the general knurl pattern that cannot be represented by any of the above. A tool_knurl is only done with special tools, the user_defined_turning_tool for knurls defined in ISO 14649-121 (ISO, 2005b).

\subsection{Specification of the base part}

In the proposed system, the initial process is to specify the base part in terms of shape and dimensions. The base part shape is then considered as the initial shape of the material. Figure 4.19 illustrates the cylindrical base part supported by the proposed system and its shows the shape, location and orientation. The base shape 
is positioned with the $\mathrm{z}$ axis parallel to the principle axis of the cylinder. The $\mathrm{x}$ and $y$ axes are orthogonal to the $z$-axis. The origin of the axes is positioned at the centre of the circular base of the cylinder.

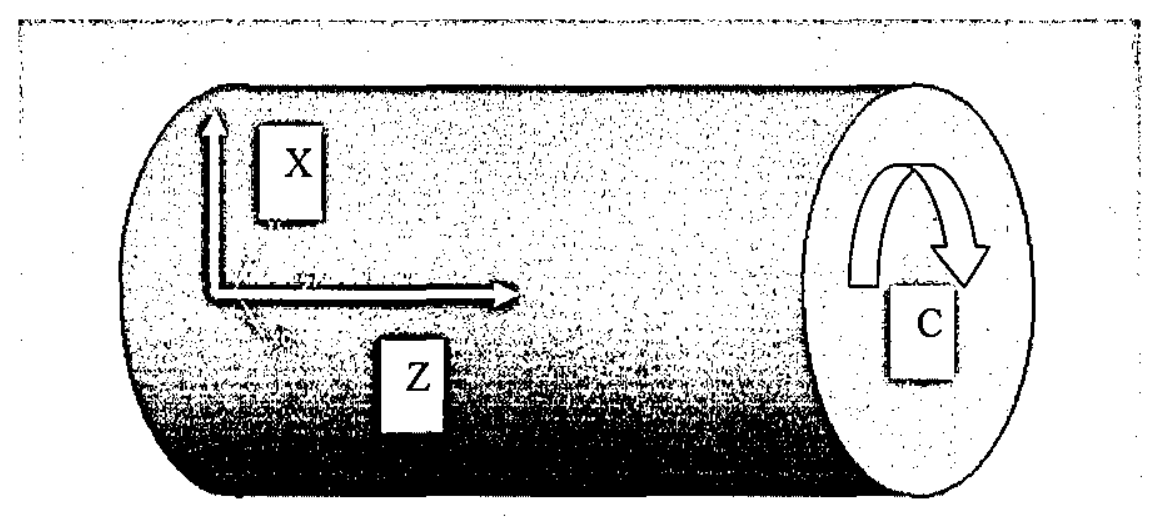

Figure 4.19 The cylindrical base part

\subsection{Machining operation}

This is the base class of all operations for turning. It includes all necessary attributes to describe technology and cutting strategy. It is a subtype of the entity machining_operation as defined in ISO 14649-10 (ISO, 2004a). If the turning_machining_operation is referenced by a turning_workingstep, the inherited attribute start_point refers to the first feature in the list of the associated turning_features. In general, there are two types of machining operations: roughing and finishing. Roughing is used to remove material from the original raw material by multiple surface passes down to the finishing allowance. Finishing then removes the finishing allowance to yield the final form of the feature. Turning_machining_operation is one of the following: facing, grooving, contouring, threading or knurling as shown in figure 4.20. Milling, drilling, boring, centre drilling and reaming not defined as subtypes of turning_machining_operation but are defined by referring to the respective operations in ISO 14649-11 (ISO, 2004b). 


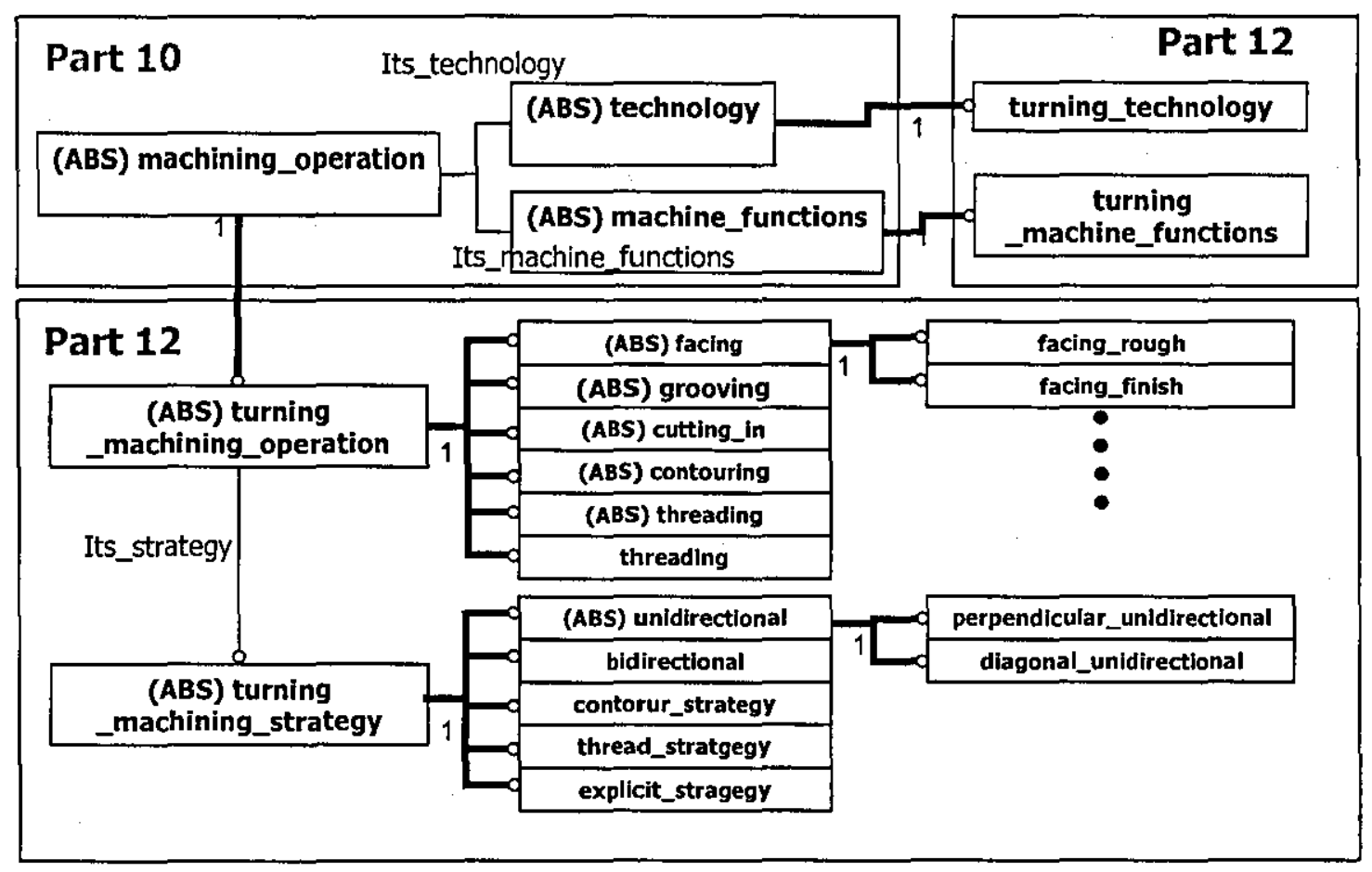

Figure 4.20 The EXPRESS-G diagram for machining_operation (ISO, 2005a)

\subsubsection{Workpiece and Manufacturing Features for Turning}

The base class of all features used for turning is the turning feature. The turning_feature is a subclass of the two5D_manufacturing_feature described in ISO 14649-10. The turning features described in this chapter are fully harmonized with ISO 10303 AP224. The entity turning feature is the abstract base class for all features used for turning. The defined turning features are classified geometric shapes that can be obtained by turning the cylindrical workpiece with 2-axes ( $\mathrm{x}$ and z) operation or 3-axes ( $\mathrm{x}, \mathrm{z}$, and c) operation as shown in figure 4.21 (ISO, 2005a). The $\mathrm{x}$ and $\mathrm{z}$ axes refer to linear movement and the $\mathrm{c}$ axis refers to rotary movement. 


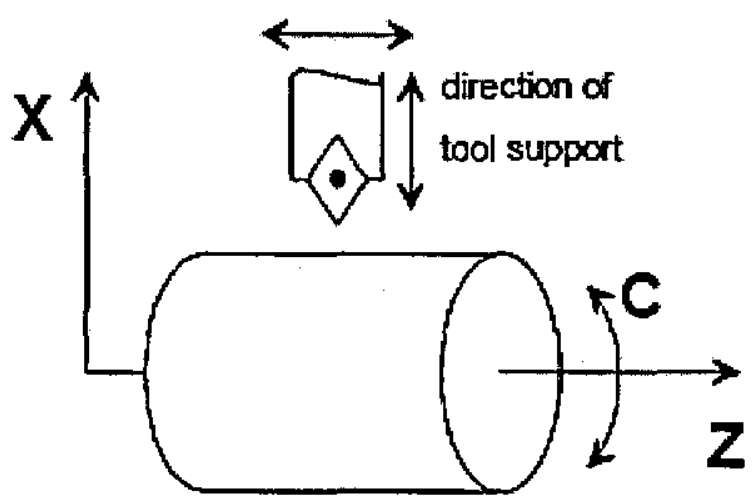

Figure 4.21 Axis and motion nomenclature of turning operation (ISO, 2005a)

\subsubsection{Turning Machining Strategy}

This entity is used as an abstract supertype (ABS) for the description of the strategy that can be used for creating a turning toolpath. It is a subtype of entity machining_strategy as defined in ISO 14649-10. All directions defined in subtypes are related to the workpiece co-ordinate system. A detailed UML representation of such bidirectional strategies adopted from ISO 14649 Part 12 is shown in figure 4.22 and 4.23 .

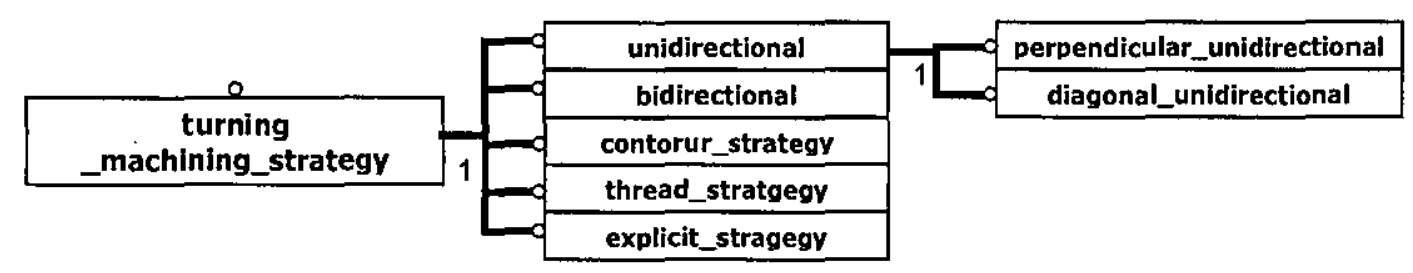

Figure 4.22 EXPRESS-G diagram for turning_machining_strategy 


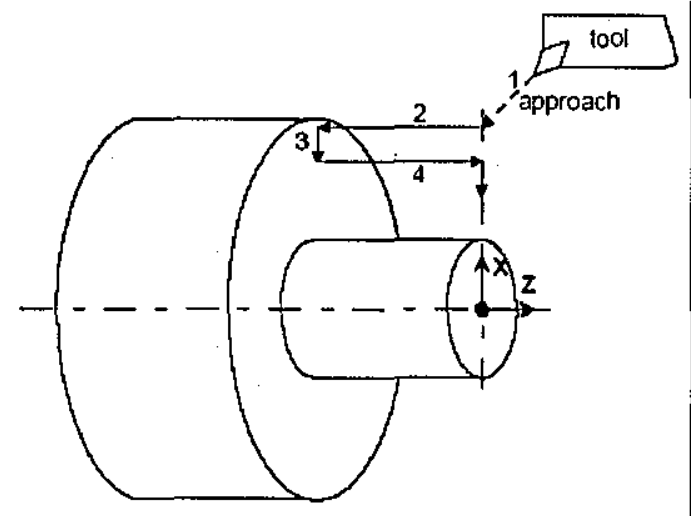

(a) feed direction: $-\mathbf{z}$

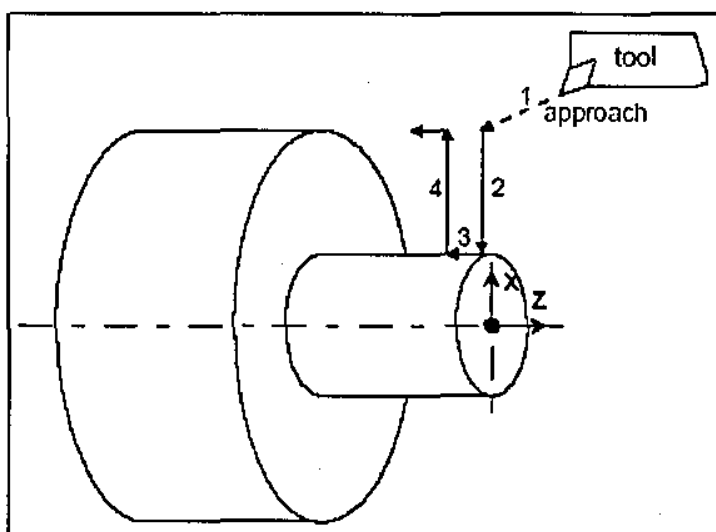

(b) feed direction: - $x$

Figure 4. 23 The turning_machining_strategy, Bidirectional $\mathrm{x}$ axis (a) and $\mathrm{z}$ axis (b) (ISO, 2005a)

\subsubsection{Turning Technology}

This entity defines the technological parameters of the turning operation. It is a subtype of the entity technology defined in ISO 14649-10 and is shown in figure 4.24. The feedrate for the $x$-axis and the $z$-axis are defined through the feedrate property or alternatively by feed_per_revolution, which is inherited from the entity's upper class. If its_adaptive_control is invoked, some or all of the values from turning_technology may be ignored. 
Chapter 4

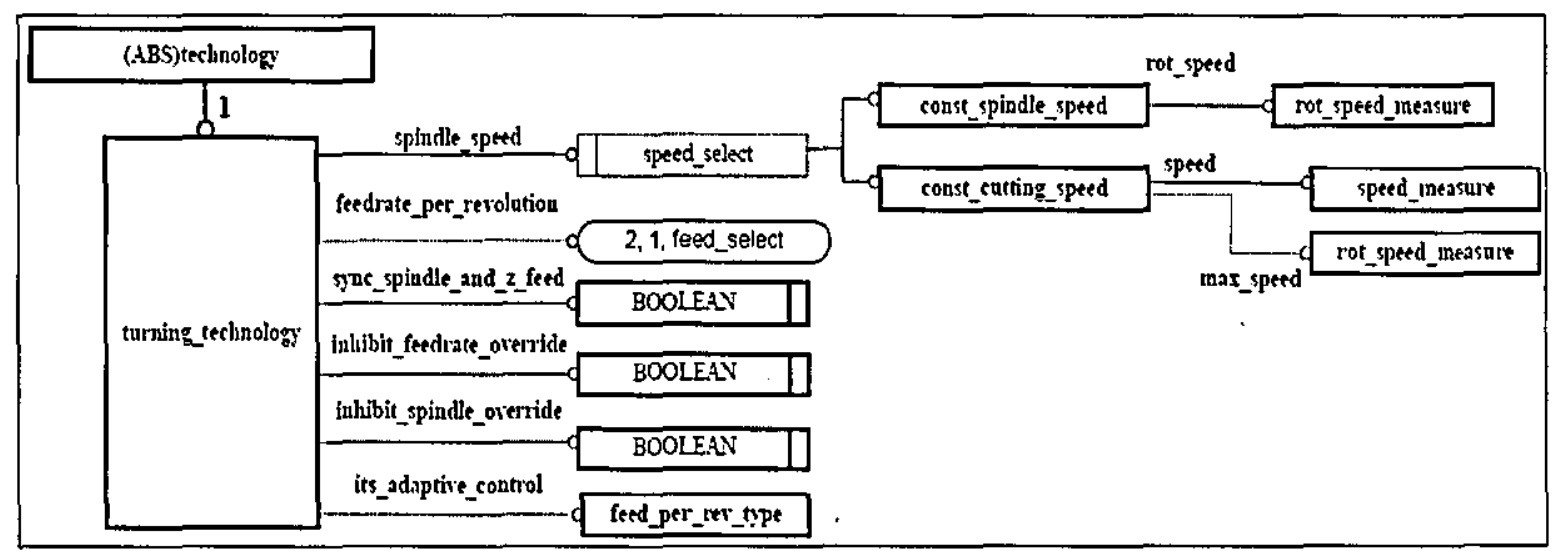

Figure 4.24 EXPRESS-G diagram for turning_technology (ISO, 2005a)

\subsubsection{Turning Machine Function}

This entity describes the state of various functions of the machine, like coolant and chip removal to be applied during the time span of an operation. It is a subtype of the entity machine_functions defined in ISO 14649-10 (ISO, 2004a). Some attributes of turning_machine_functions are the same as those in milling_machine_functions which are defined in ISO 14649-11 (ISO, 2004b). Referring to ISO 14649 Part 12 (ISO, 2005a), turning machine functions involve machine functionality such as the coolant being either active or not and the type of coolant.

\subsubsection{Turning Machining Operation}

This is the base class of all operations for turning. It includes all necessary attributes to describe technology and cutting strategy as shown in figure 4.25 . It is a subtype of the entity machining_operation defined in ISO 14649-10 (ISO, 2004a). If the turning_machining_operation is referenced by a turning_workingstep, the inherited attribute start_point refers to the first feature in the list of the associated turning_features. Turning_machining_operation is one of the following: facing, grooving, contouring, threading or knurling. 


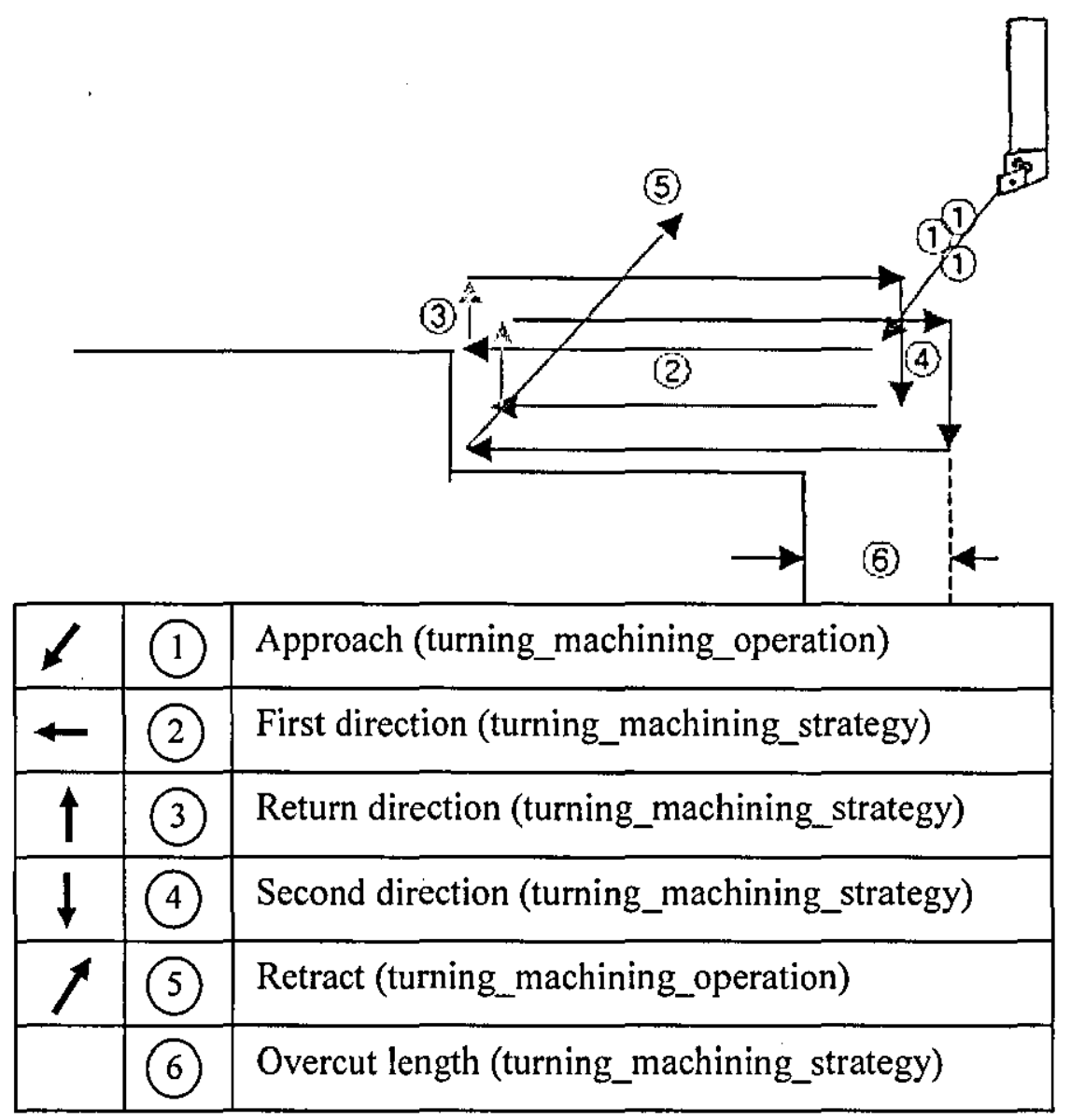

Figure 4.25 Turning machining operation and strategy schema

\subsection{Turning Machine Tool Database}

This entity describes the technology-specific information needed for the description of cutting tools for turning machines. It is a subtype of entity cutting_tool defined in ISO 1464 Part 10 (ISO, 2004a). As illustrated in Figure 4.26, overall_assembly_length means the total length of holder including any portion in front of the cutting point, and overall_assembly_width means the total width of the holder including any portion in front of the cutting point. The definitions are valid for other types of turning machine tools, such as those shown in Figure 4.27 and overall tool assemblies referenced from ISO 14649 Part 121 (ISO, 2005b) shown in figure 4.28. 


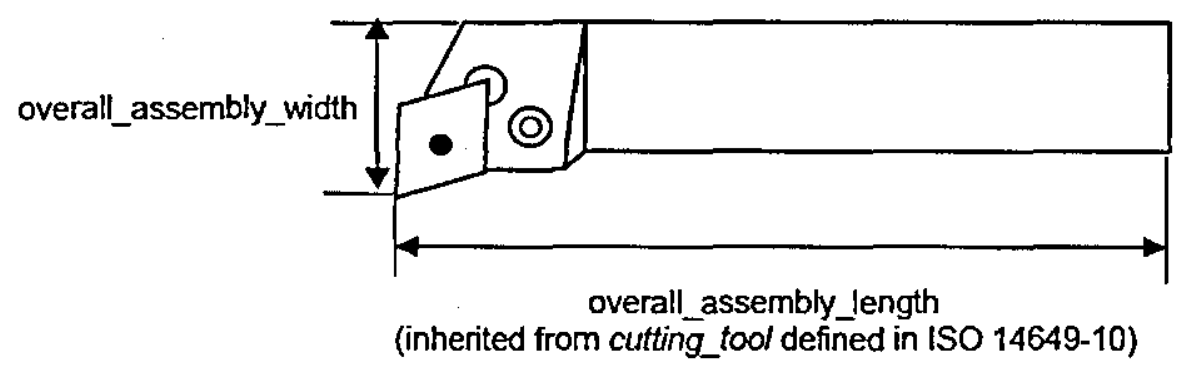

Figure 4.26 Overall tool assembly (ISO, 2005b)

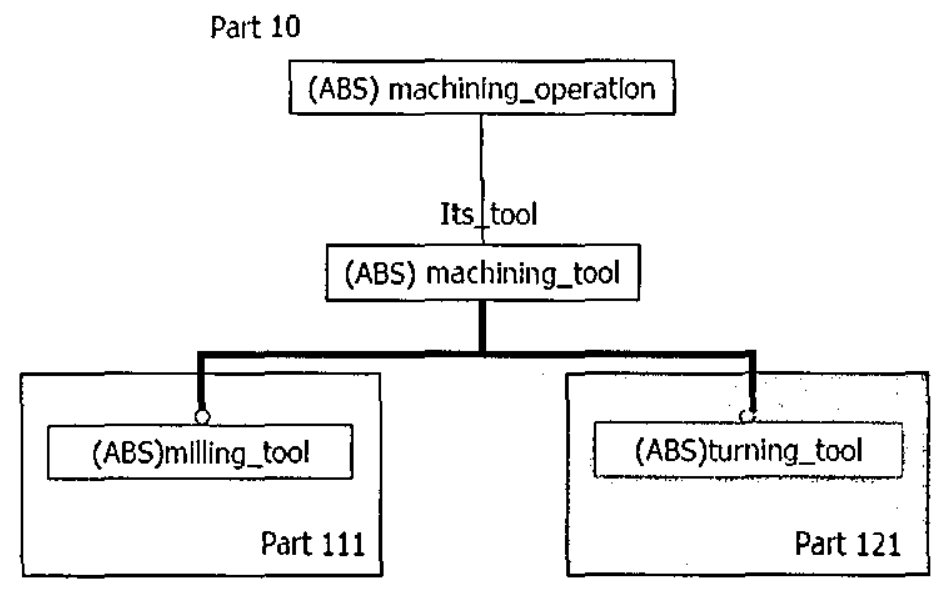

Figure 4.27 Machine tool (ISO, 2005b)

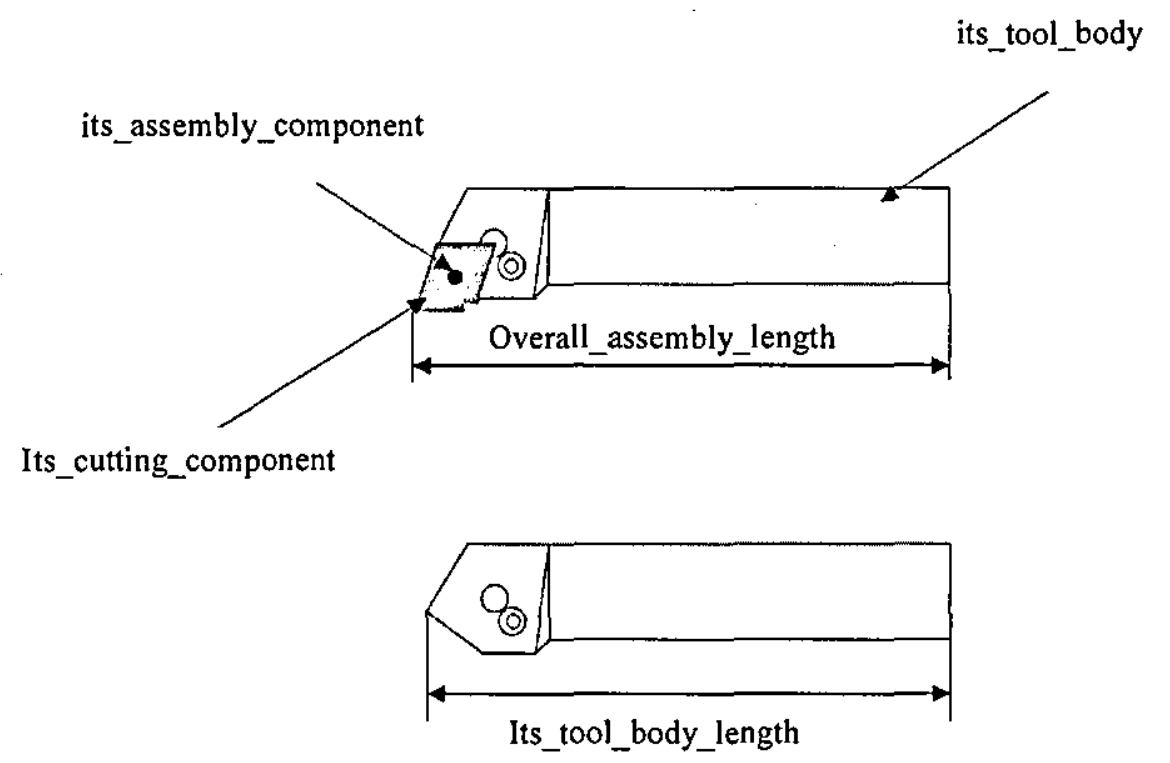

Figure 4.28 Overall tool assembly (ISO, 2005b) 
The entity Turning_machine_tool_body is the supertype for all types of turning tools which are the general_turning_tool, knurling_tool, turning_threading_tool, grooving_tool and the user_defined_turning_tool. With this set of entities STEP-NC can describe most of the turning tools used in industry. Figure 4.29 describes the Turning_machine_tool_body entity. The majority of the parameters in the Turning_machine_tool_body entity are optional with the exception of the dimension parameter defined by the Turning_tool_dimension entity.

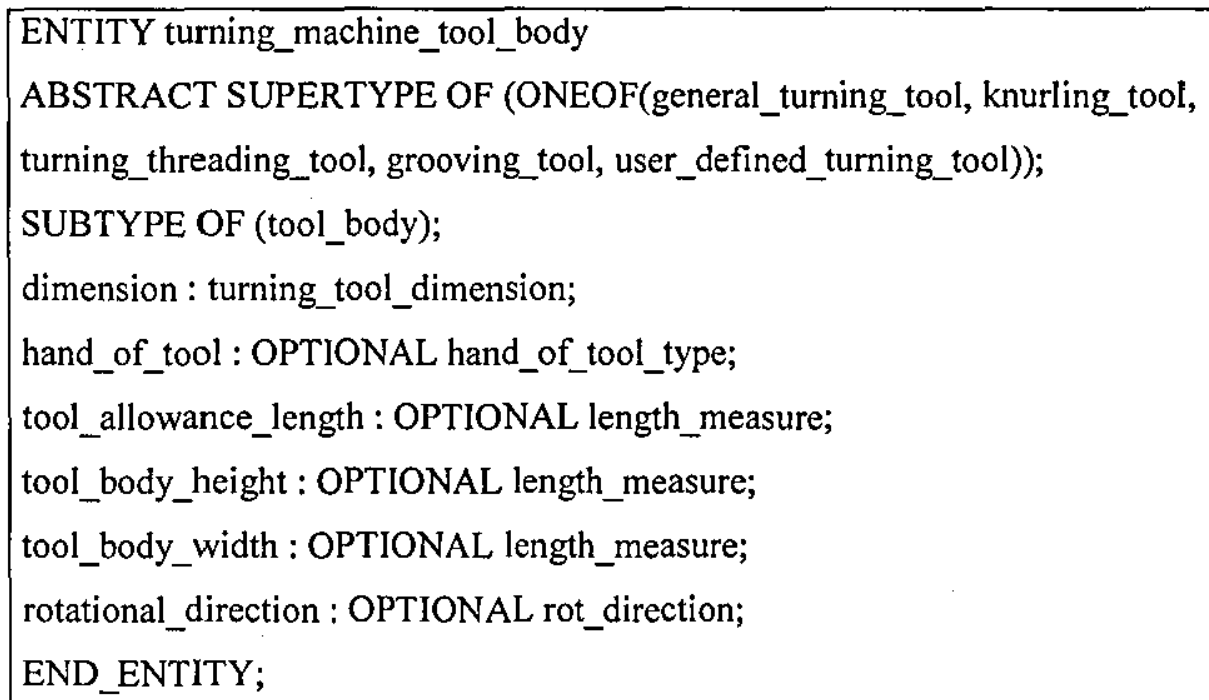

Figure 4.29 - Turning_machine_tool_body entity (ISO, 2005b)

\subsection{Process Plan Generation}

A pre-processor has been developed to generate a physical STEP-NC process plan file. The main function of this pre-processor is to receive information related to the workpiece (features, tolerances, material, etc.) and information produced by process planning (machining operations, cutting tools, machining parameters, etc.) to generate a STEP-NC process plan. STEP-NC, ISO 14649, consists basically of feature-based process plans for manufacturing processes such as milling and turning operations. To date STEP-NC has been developed either for 
milling or turning operations based upon the feature information of ISO 10303AP224 (ISO, 2000b). There have been no attempts to handle turn/mill operations.

\subsection{Output Structure}

A part program is described in a Physical File Format according to ISO 10303-21 (ISO 10303 Part 21) and has two sections namely "Header" and "Data" as shown in figure 4.30. As mentioned before, the proposed SCSTO is based on ISO 14649 , which is related to ISO 10303. Output is specified in an exchange structure format using ISO 10303 Part 21 representation for product data of the STEP AP 224 schema which is specified in the EXPRESS language (STEP Part 11) (ISO, 2002). The file format is suitable for transferring product data among computer systems and can be edited by users.

\subsubsection{Header Elements}

Header elements contain information that is applicable to the entire exchange structure, and are present in every exchange structure. Three header elements are specified and each is required to occur exactly once in every exchange structure. The header elements are:

i. The file description specifies the version of this part of ISO 10303 used to create the exchange structure as well as its contents.

ii. The file name provides human readable information about the exchange structure.

iii. The file schema indicates the EXPRESS schemas that specify the entity instance in data elements. 


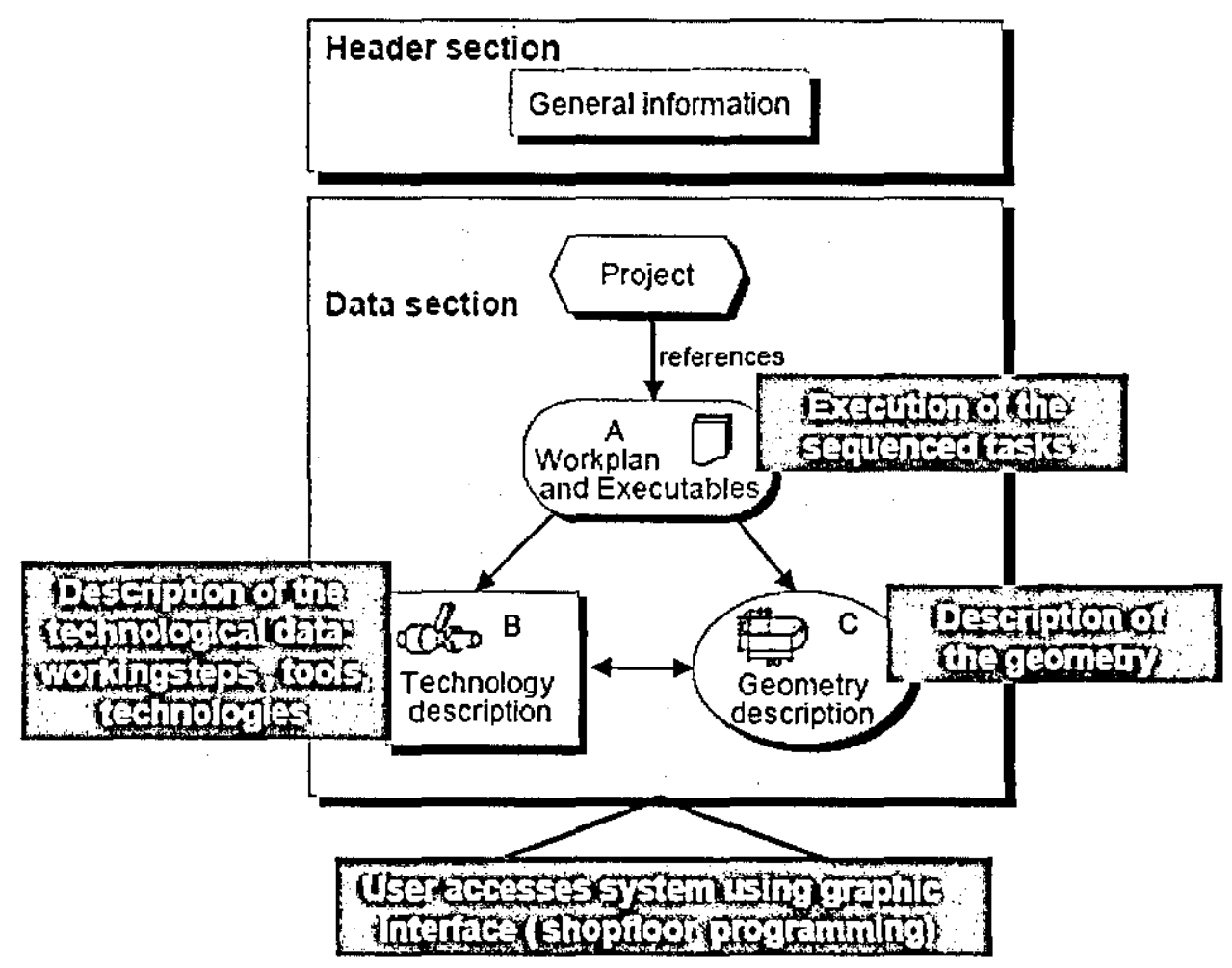

Figure 4.30 Part of STEP-NC structure format (Muller, 2000b)

\subsubsection{Data Elements}

Data elements contain the product data to be transferred by the exchange structure. The data elements are instances of entities that correspond to the EXPRESS schemas governing the exchange structure;

i. The part properties contain the description of characteristics of the part that is being defined. These characteristics specify requirements for manufacturing that apply to the state of the part at a particular time prior to or after the manufacture of the part.

ii. The base shape specifies the size and shape of the initial stock from which the part is to be produced. The placement element which specifies the location and orientation of the part is also included. In this thesis the base shape is restricted to be cylindrical. 
iii. Administrative data contains information that identifies the product data order of information.

iv. The machining feature element contains the information necessary to identify shapes which represent the volumes of material that shall be removed from the part by machining or shall result from machining. This will be repeated in the structure according to the number of machining features that exist in the part.

v. The placement element specifies a set of axes and positions to place the feature in the part.

vi. The feature definition element contains the information necessary to create a machining feature.

The main element of the data section of the program file consists of all information about; i) workplan and executables, ii) geometry, and iii) technology descriptions (STEP-NC-Project, 2000).

\subsection{Summary}

This chapter has highlighted the framework of the proposed system based on ISO 14649 Part 1, Part 10, Part 12 and Part 121 (ISO, 2003b; ISO, 2004a; ISO, 2005a; ISO, 2005b) and has described tools that are used for the STEP Compliant CAPP/CAM system for turning operations. SCSTO aims to address the process planning and machining of turning discrete components and generation of ISO 14649 code which can be used for turning component manufacture. The fundamental aspects of the machining process plan and a design of the framework for SCSTO has been provided. The following chapter presents the STEP-NC compliant information model. 


\section{Chapter 5 - STEP-NC Compliant Information Modelling}

\subsection{Introduction}

This chapter outlines the research development in terms of STEP-NC compliant information modelling. Information from traditional CAD systems is largely limited to the representation of geometric data but the work presented here may be seen as intended to serve as the precursor for STEP in the lifecycle of a product and is used to capture relevant information. As discussed in Chapter 2, STEP is used for the exchange of information that is the outcome of design activities and in this context conceptual information models form the basis of the work. From previous research it is clear that an information model can be represented using different techniques, such as EXPRESS-G (Schenck and Wilson, 1994), the Unified Modelling Language (UML) (Booch, 1999), the Entity-Relationship (ER) (Chen, 1976) and Nijssen's Information Analysis Method (Nijssen and Halpin, 1989) as well as EXPRESS (ISO/TC184/SC4, 1994; (Schenck and Wilson, 1994).

In this research the focus is on UML as it is a universal format that is familiar to most business applications, and a comprehensive framework for the proposed system was presented in the previous chapter. The information model represents the manufacturing capabilities of the turning operations by implementation of both a Product Model and a Manufacturing Model using the combined methodologies of the Integration Definition for Function Modelling (IDEF0) and UML. This research work applies the object oriented approach to construct the product model and corresponding design and process planning function models. The object oriented programming language, Java programming (Landy et al., 2003), UML and Rational Rose 2005 are used to implement the proposed data model and process planning functions. 
The basic consideration for the design of the structures was that they must allow the use of the models as a source and repository for the information required during the product development cycle by software applications running concurrently. The rest of this chapter describes the process followed in order to define the general structures of the information models and mainly focuses on the development of the model that is required for SCSTO.

\subsection{Overview of Modelling Languages}

A modelling language in this thesis refers to any artificial language that can be used to express information in a structure that is defined by a consistent set of rules. Interpretation of the meaning of the components in the structure may be either in graphical or textual form. The graphical form refers to diagrammatic techniques with concepts represented by symbols and relationships and constraints represented by lines. The textual form refers to standardized keywords accompanied by parameters to make computer interpretable expressions. The following section in this chapter is concerned with modelling itself and it consists of product and manufacturing information. As stated in Chapter 4, this model refers to the STEP-NC environment and is involved with the product, process and resources as summarised by Larson, (2006). The model can be divided into the three aspects of product model, process model and resources model (Larsson, 2006). The Product model can be classified as a model that describes the product of a process, e.g. its type, structure, properties and the relationship between its components. A Process model refers to the part of the lifecycle that deals with production and maintenance of the product, as opposed to the management process. Finally, the resource model can be described as a model describing resources either needed or supplied to a process, e.g. their types, relations, structure and properties.

One of the constructors in information modelling in the EXPRESS language is EXPRESS-G. EXPRESS-G is a graphical notation for the display of information models. The notation only supports a subset of the EXPRESS language and therefore EXPRESS-G models are normally abstractions of EXPRESS models. To understand the flow of application objects and to identify where the appropriate data is coming from or going to, outer_round has been chosen as an example as shown in figure 5.1 (EXPRESS-G diagram). Outer_round is found in the STEP AP224 documentation 
(ISO, 2000b). It is clearly stated that outer_round, has two subtypes that are outer_diameter_to_shoulder and outer_diameter. An outer_diameter_to_shoulder feature is defined by a shape boundary ( $v_{-}$shape_boundary) from the vee_profile. object. Similarly the outer_diameter feature is defined by the three parameters diameter, feature_length and reduced_size.

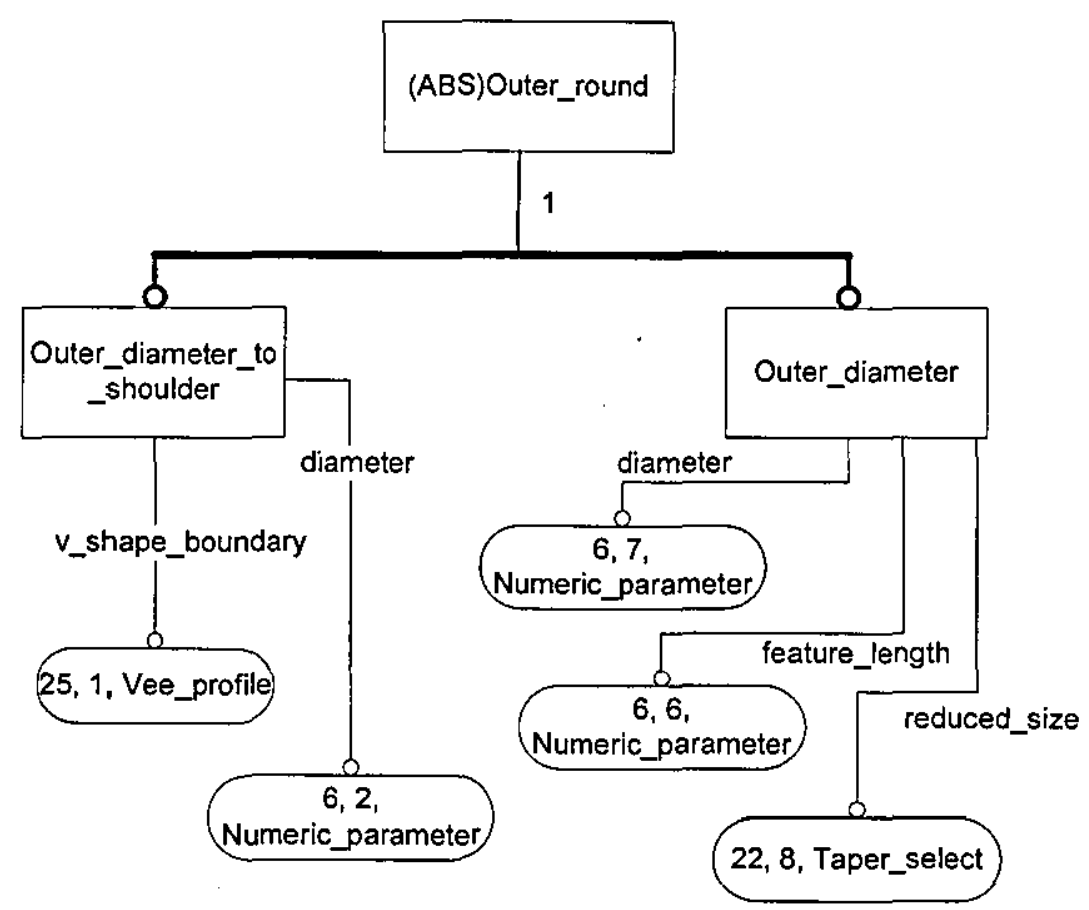

Figure 5.1 Express-G diagram for the feature outer_round (ISO, 2000b)

\subsection{Information Model}

The information modelling approach has the ability to offer the information in a readily usable manner. The structure and classification of information is important, if information as to be readily found and used (Young, 2003). According to ISO a model is a representation or description of an entity or a system, describing only the aspects considered relevant in the context of its purpose (ISO, 2003a). An information model needs to provide the explicit interpretation rules that are required (Nielsen, 2003). Most manufacturing models concentrate on representing manufacturing resources and their combination into manufacturing processes (Giachetti, 1999). The manufacturing resources are the tools, fixtures and machines that are combined and arranged into a manufacturing process to fabricate a product. 
The implementation of SCSTO was build up of three main stages, namely the representation of the information model, the development of the tool database and the construction of the system application. Figure 5.2 shows an information model of the proposed system. The first stage starts with the proposed system framework for SCSTO including the information model derived from the STEP-NC standards. The framework is described mainly by the information and functional perspectives of the CAD to CNC process chain. The information model was established by Molina (Molina, 1995) and consists of product and manufacturing models. The product model represents relevant information about the product throughout its life cycle while the manufacturing model is defined as an information model that identifies information describing the manufacturing resources, processes and strategies.

The author's information model for the product involves the bar (material), manufacturing perspectives and design. On the other hand, the manufacturing model includes resources, operation and strategy. The system is modelled by a UML diagram and clearly represents the system information model in terms of classes, attributes, relationships and operations. Each of the entities is based on ISO 14649 Parts 10, 11, 12, 111 and 121 (ISO, 2004a; ISO, 2004b; ISO, 2005a; ISO, 2005b; ISO/FDIS, 2004) 


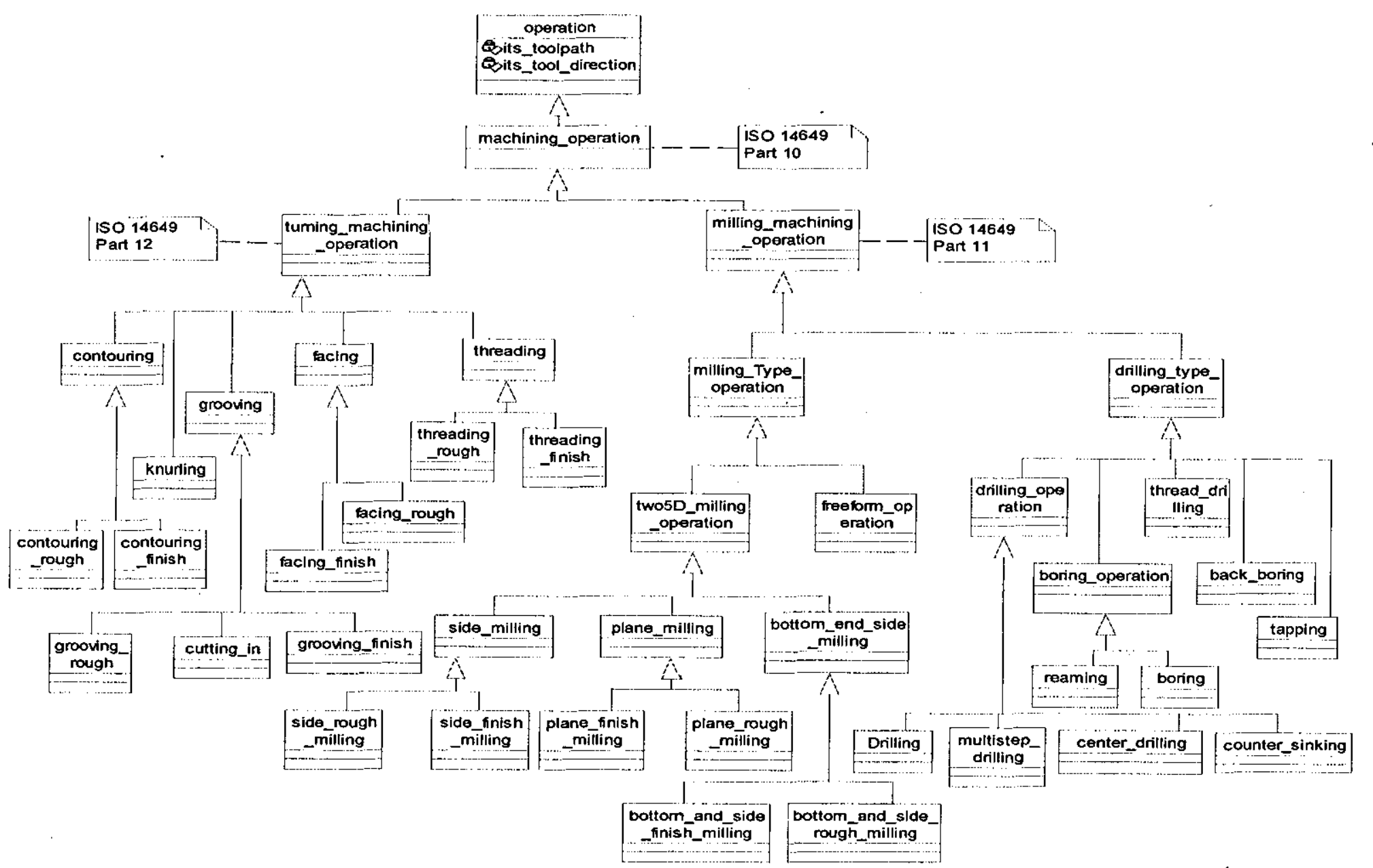

Figure 5.2 Various objects in the SCSTO environment and the relationships 
The information such as manufacturing features, tool requirements and the manufacturing process can be described as "what-to-do" information, meaning only manufacturing tasks are described. Wang and $\mathrm{Xu}$ called generic STEP-NC information "how-to-do" information (Wang and $\mathrm{Xu}, 2004$ ) as it is hardware independent and is used in generating process plans. Both types of information are based on ISO 14649-1 (ISO, 2003b) as shown in IDEFO figure 5.3.

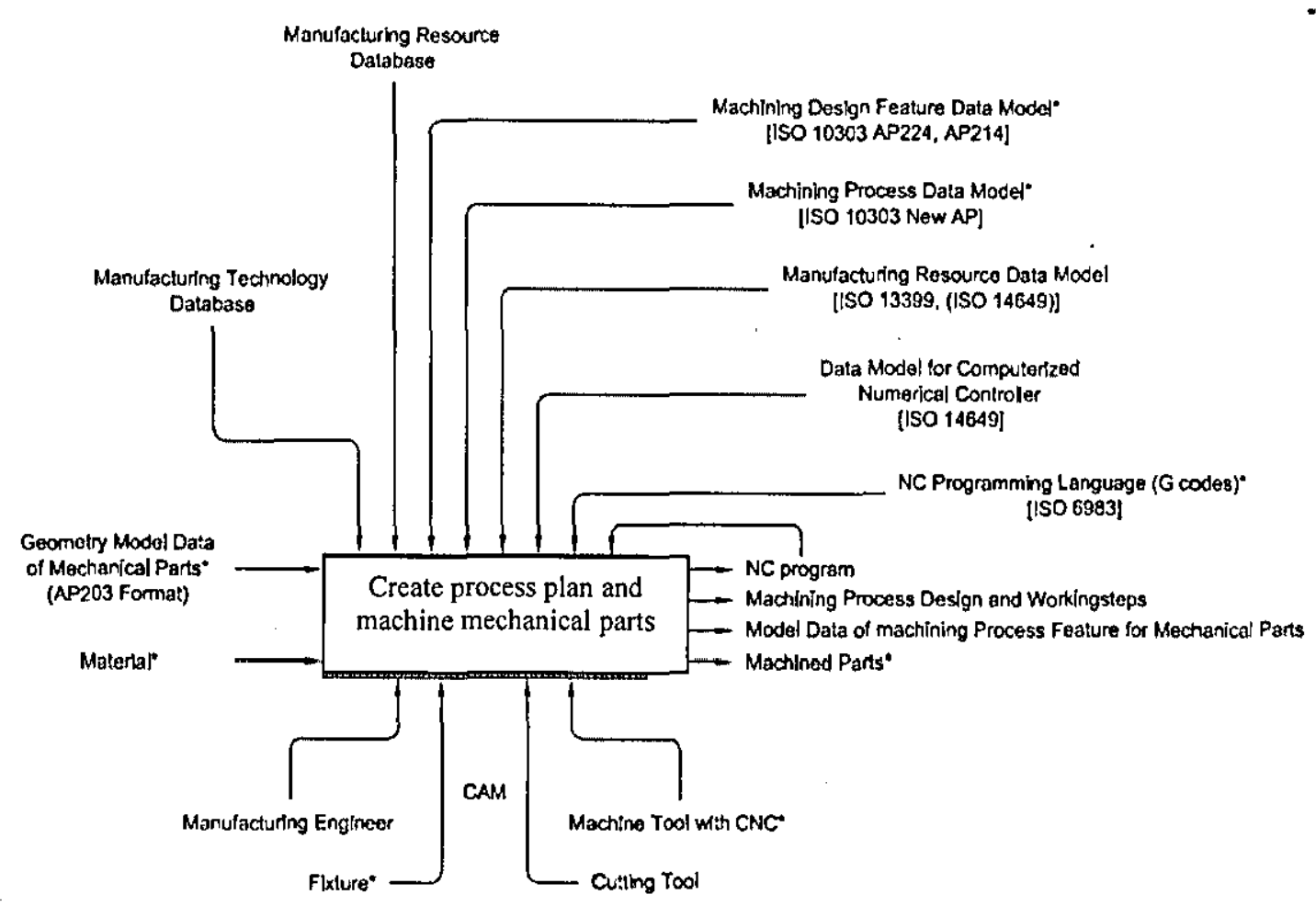

Figure 5.3 Digital manufacturing based on ISO 14649-1 (ISO, 2003b)

The second aspect of SCSTO is a database system developed using JdataStore (details discussed in Chapter 6). In this system all the cutting tools that are involved in SCSTO are either turning or milling tools. The process of developing a database starts with creating a file, establishing a network connection, using a test query to confirm connection and development of a database.

A graphical user interface (GUI) is the following stage for SCSTO and was created using JBuilder 2005 based on java programming (Landy et al., 2003) and is described in Chapter 6. The JBuilder essentially contains classes of related functionality that are used to develop the graphical user interface (GUI), to access the database and to identify the inputs and outputs of SCSTO. 


\subsubsection{The Product Model}

In the design stage, the expressions and circumstances of the real world are transferred into an abstract model. This model has to fulfil a number of requirements. Besides the requirements resulting from the analysis stage, there are also some basic requirements like clarity, maintainability, extendibility and robustness. Also it must satisfy the aspects of object-orientation. The main parts of an object-oriented model are;

i. basis elements (classes, objects, attributes, methods, ...)

ii. static elements (associations, aggregations, generalizations, ...)

iii. dynamic elements (conditions, activities, ...)

The product model is contained within a set of interconnected objects. The class definition of these objects is based on the entity definition that exists within ISO 14649. In this case, the product model facilitates the management of information representing the stock material, in terms of the material and size, and the design represented by the dimensional and geometric tolerances. In addition, the manufacturing perspective of the part is included in the product model in order to serve as a means of integrating the product and manufacturing models as shown in figure 5.4. (Turning features are marked on this diagram as being of particular interest).

The manufacturing perspective supports the preliminary and interoperable process planning in the early product development stage in an integrated manufacturing environment. 


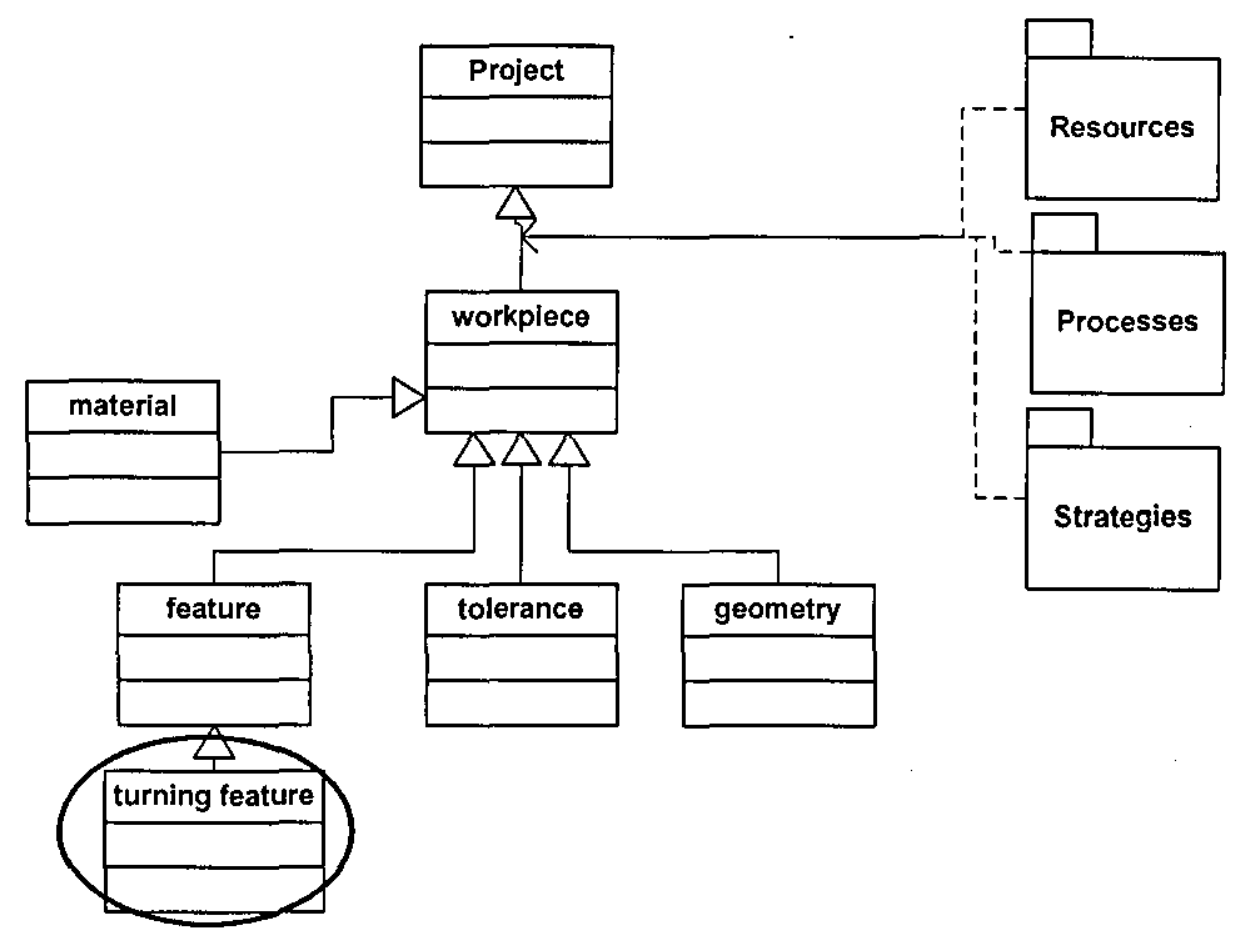

Figure 5.4 Representation of STEP-NC compliant model for turning - product data model

\subsubsection{The Manufacturing Model}

A comprehensive framework for a manufacturing model which encompasses processes, resources and strategies for factories, shops, cells and stations is described by Molina but in this research the focus is on workstations (Molina, 1995). The overall manufacturing model is shown in figure 5.5 (Rosso-Jr, 2005) and, consists of;

i. Process characteristics that have been modelled in a generic format. Reference to form makes the nature of the description process- specific.

ii. A mapping between manufacturing processes and manufacturing resources has been established and used. This enables the identification of the appropriate resources in a manufacturing facility to support product manufacture.

In this research the manufacturing model has been made up of the following attributes;

Turning resources - model the physical constraints imposed on the turning process to machine the required part such as turning_tool and machine. 
Turning processes - model the functional capabilities of the turning process to perform the machining operations such as workpiece_setup and turning_machining_operations.

Turning strategies - model the machining schemas of the turning process on the given turning resources and processes such as turning_approach/retract_strategy and turning_technology.

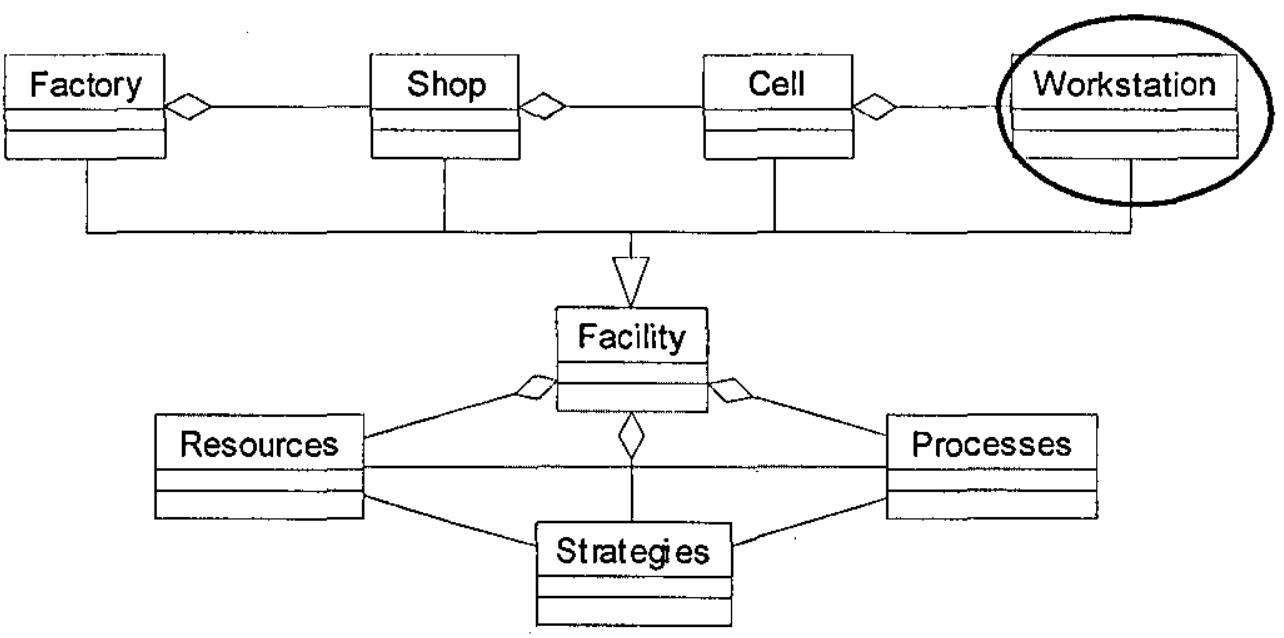

(a) The top entities in the Manufacturing Model with a modified Molina's (1995) structure

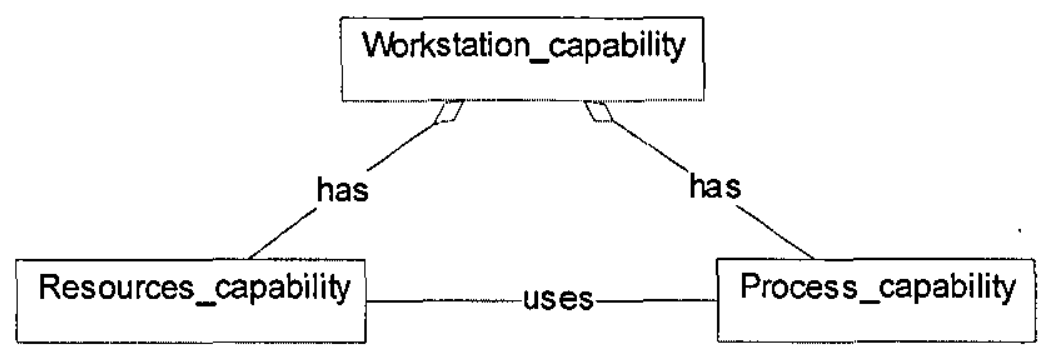

(b) The top entities in the Manufacturing Model

Figure 5.5 The Manufacturing Model representations of (Molina, 1995) and (Rosso$\mathrm{Jr}, 2005$ )

Finally the author has proposed a manufacturing data model compliant with STEP-NC as shown in figure 5.6, that is specifically directed at turning machining operations and drilling machining operations. 


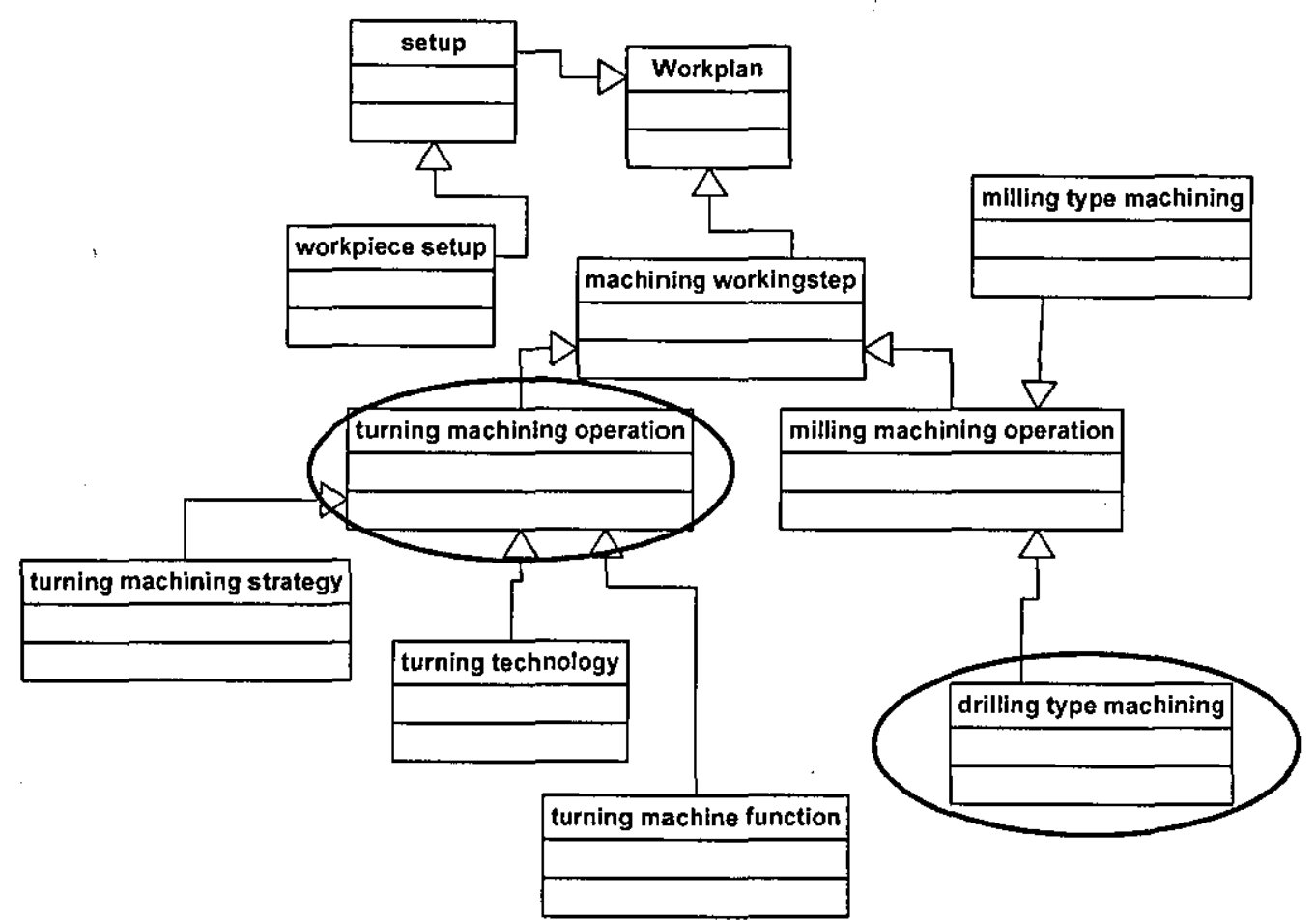

Figure 5.6 Representation of STEP-NC compliant for turning - manufacturing data model

\subsection{ISO 14649 Classes}

The ISO 14649 process data for turning is Part 12 (ISO, 2005a), which specifies the technology specific data elements that are needed as process data for turning (ISO, 2005a). Part 10 (ISO, 2004a) is general process data which describes the interface between the computerized numerical controller and the programming system such as a CAM system or a shop floor programming system for turning. It can be used for turning operations on all types of machines including turning machines (lathes) or turning centres. Included in part 12 are features and operation data models for conventional turning involving $\mathrm{x}$ and $\mathrm{z}$ movements. This again only represents the standard rotational turning with no representation for features and operations for composite machining such as $\mathrm{C}$-axis milling operations. Figures 5.7 and 5.8 show the example turning classes based on ISO 14649. This system needs integrated manufacturing information about the product model and manufacturing resources, and is also based on an object oriented platform. Another aspect of information is 
describing the manufacturing process and the product geometry that can be created and manipulated.

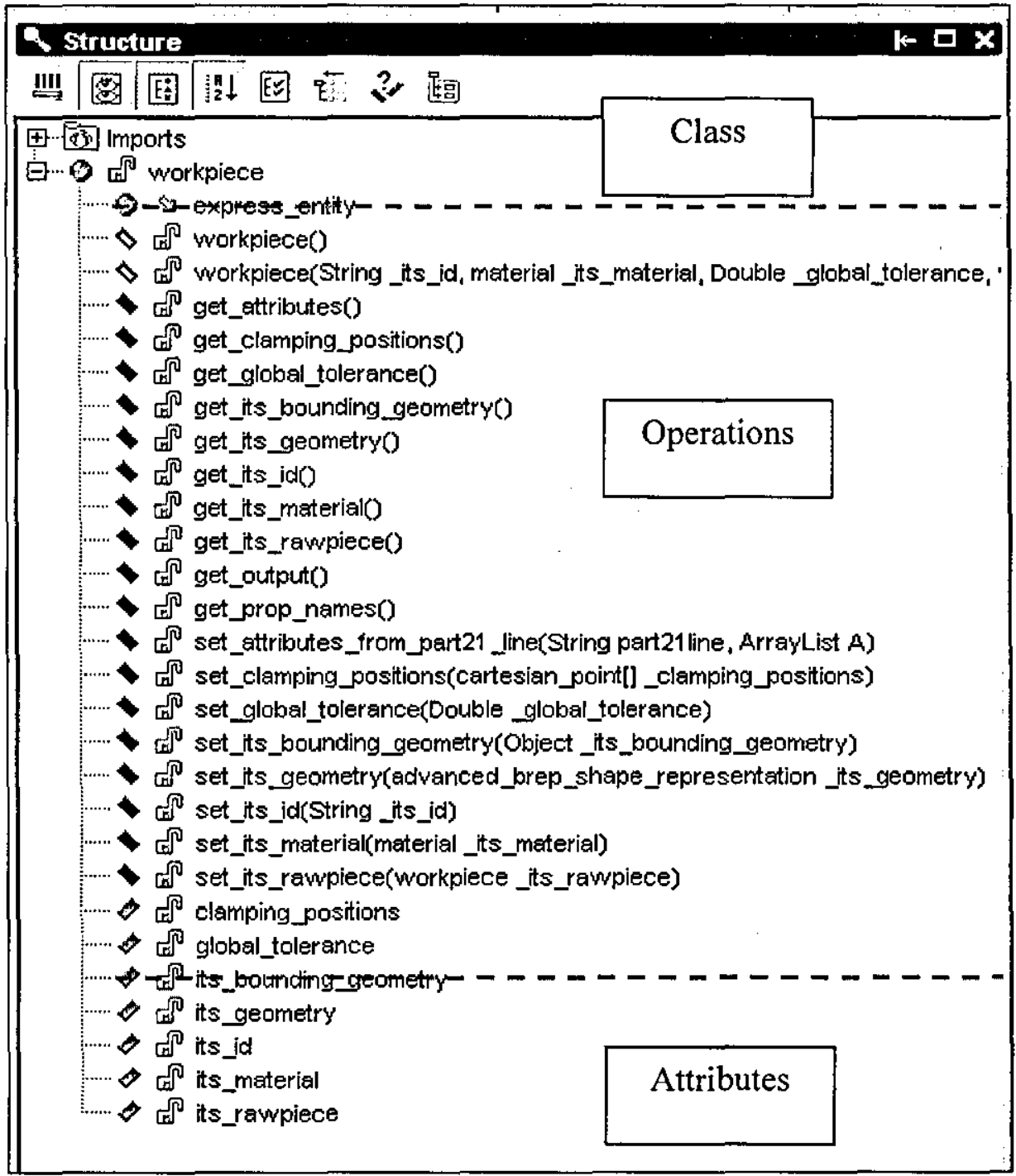

Figure 5.7 Workpiece class diagram

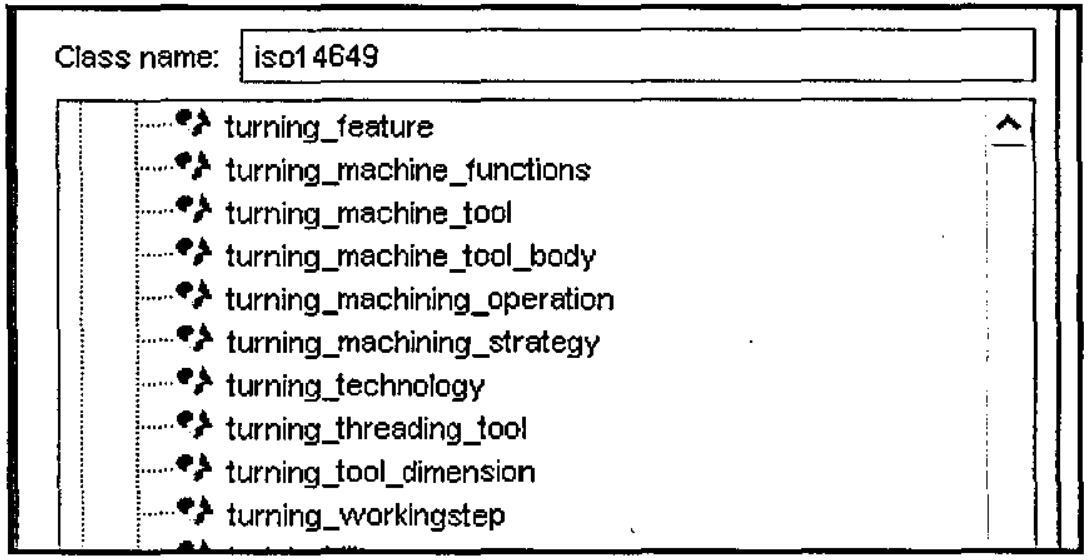

Figure 5.8 Turning classes 
The structured model approach, in the STEP-NC manufacturing chain, starts with the definition of the feature-based design geometry in a CAD/CAM system. An ISO 10303 Part 21 physical file (ISO, 2002) is then generated from a STEP-NC Compliant CAPP/CAM system based on a suite of JAVA information classes from the STEP-NC ARM model definition, developed by Loughborough University (Nassehi et al., 2006a). The user is able to define STEP-NC features and is prompted for associated manufacturing inputs such as workingsteps, operations, tools, feeds and speeds consistent with the STEP-NC ISO 14649 Parts 12 and 121 standards (ISO, 2005a; ISO, 2005b). The ISO 10303 Part 21 physical file (ISO, 2002) is automatically generated. This file is processed by the STEP-NC translator (developed by ISW, Stuttgart and Siemens) and is converted into the Siemens proprietary format .MPF file (Nassehi et al., 2006a). The generated file can then be directly machined on any CNC workstation equipped with a Siemens controller and ShopTurn CAM software.

\subsection{Turning Operation}

The turning schema of the ISO standard provides definitions of technology specific data types representing machining features and processes for turning operations on lathes (ISO, 2005a). The turning operation has two basic categories of machining operations; either roughing or finishing. All the turning operations are under the machining,operation sub class which is based on the operation class. Figure 5.9 shows an example of the turning machining operation under the EXPRESS Schema and the class diagram of facing objects from the entity definitions within ISO 14649. In turning, the workingsteps include manufacturing features and machining operations defined by turning_feature and turning_operations respectively. 


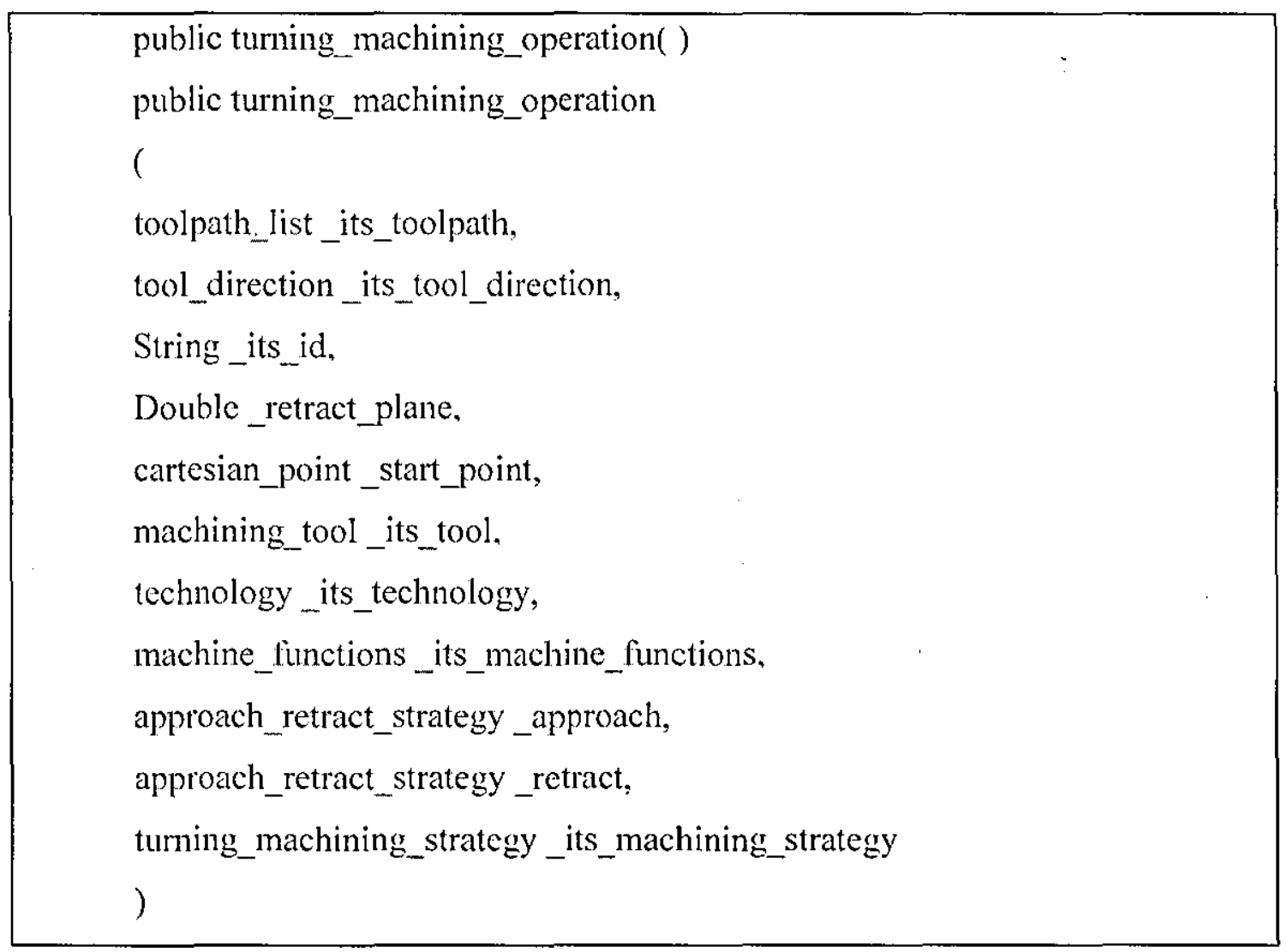

Figure 5.9 EXPRESS Schema for turning_machining_operation

\subsection{Turning Feature Library}

Manufacturing features geometry data contains the information necessary to identify shapes which represent volumes of materials that shall be removed from a component by machining or shall result from machining. To extract a rotational feature manufacturing features with their attributes from the ISO 10303 AP 224 document (ISO, 2000b) are used to make the feature a member of the turning feature library (TFL) and construct the manufacturing features and part geometry data component of the feature definitions. The Java language is used to define each turning feature class based on class, operation and attributes.

In normal practice, object oriented concepts are accepted to be suited to engineering activities because object structures are readily able to model the real world, support communication and provide interfacing and manipulation of different data tyres (Motavalli et al., 1997). Based on studies by Fidan, the object oriented representation gives the flexibility to define the system in a hierarchical manner, (Fidan, 2004) and provides the following characteristics:

i. Features in the feature definitions library can be represented as objects. 
ii. Functions of the features in the feature definitions library can be represented as methods on objects such as drawing functions or export to STEP functions.

iii. The relationships between features in the feature definitions library can be represented as messages passed between objects.

iv. System architecture can be represented by the use of UML diagrams and class hierarchy.

Based on object oriented capability, the author has created a turning feature library (TFL) in the form of a file of code containing objects that can be called from other executable codes. The TFL is arranged according to the turning feature parent. For example the outer_diameter is a child of outer_round.

Referring to ISO 14649-12 (ISO, 2005a), the following features are currently included: outer_round (outer_diameter, outer_diameter_to_shoulder), revolved feature (revolved_flat, revolved_round, groove, general_revolution), knurl (straight_knurl, diagonal_knurl, diamond_knurl, tool_knurl). Features supported by SCSTO are restricted to those based on STEP AP224 (ISO, 2000b) which was developed for mechanical product definition for computerised process planning using form features. Figure 5.10 shows the structure of the turning feature entity within the manufacturing_feature class (ISO, 2004a; ISO, 2005a).

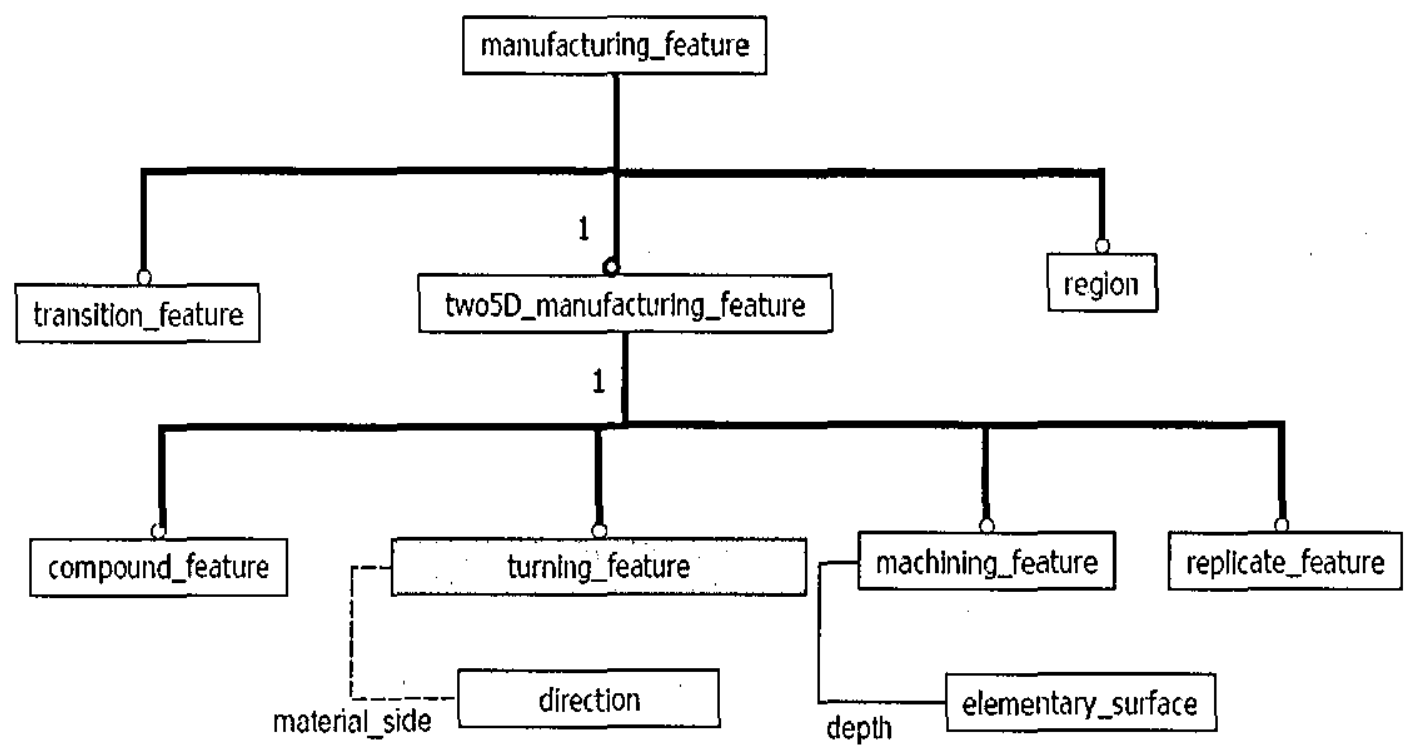

Figure 5.10 Location of turning feature adopted from ISO 14649 Part 10 (ISO, 2004a) 
A manufacturing feature identifies the types of features necessary to manufacture a component. Each manufacturing feature is either a transition feature, a two5D_manufacturing_feature or a region. Each transition_feature is either an edge_round, fillet or a chamfer. Each region class has another three subclasses i) region_projection, ii) region_surface_list and iii) topological_region. In this research the focus is on two5D_manufacturing_features and more specifically turning_features.

Figure 5.11 shows the UML format representation of ISO 14649-12: Process Data for Turning. This standard represents the turning feature within the abstract subtype (ABS) two5D_manufacturing_feature and also within the outer_round, revolved_feature and knurl subtypes. An outer_round is a type of turning feature that is an outline significant shape that is swept through a complete revolution about an axis and either is an outer_diameter or an outer_diameter_to_shoulder.

A revolved feature is a type of turning feature created from the sweep of a planar shape one complete revolution about an axis. Each revolved_feature is one of the following; revolved_flat, revolved_round, groove or a general_revolution.

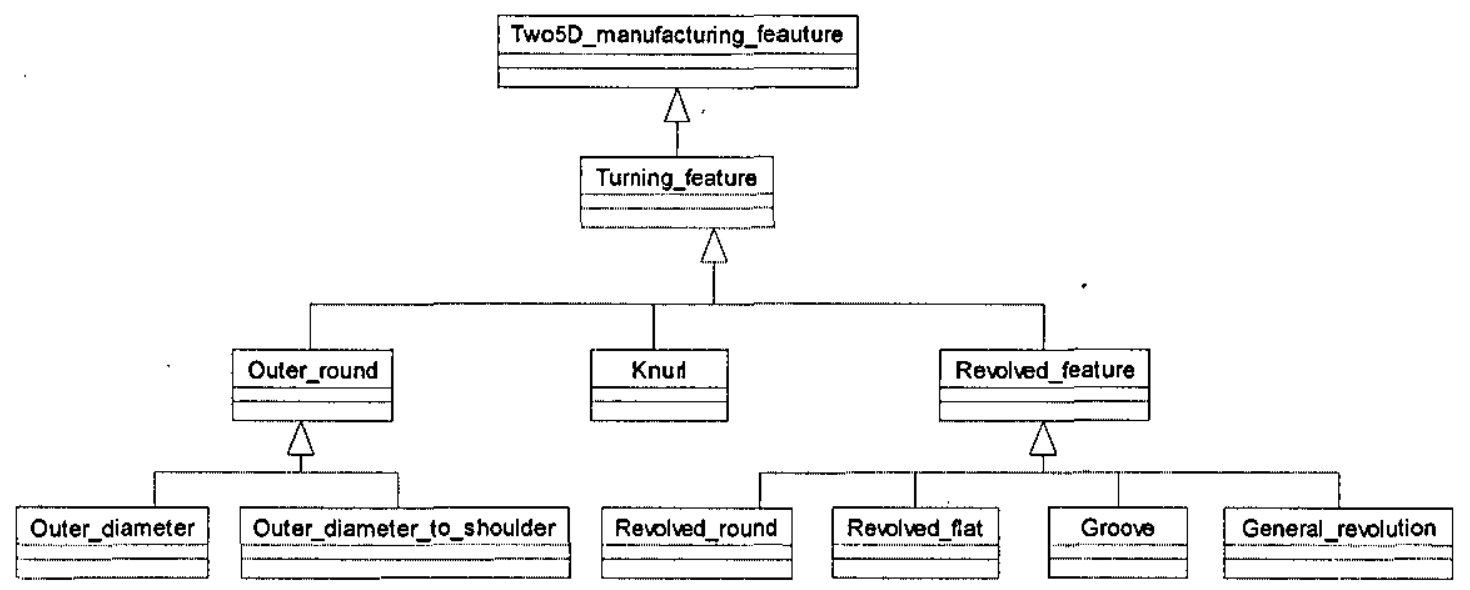

Figures 5.11 UML format representation of ISO 14649-12: Process Data for Turning.

The manufacturing feature object in figure 5.12 shows a class diagram illustrating how the object identifies itself through an ISO 14649 package. A package describes manufacturing features consisting of fields, constructors and methods using a Java programming approach. In order to develop the system a set of computing tools will be used. The Java programming language is used for the actual development of software components based on the object oriented methodology and UML is utilised as the modelling language. 
public class manufacturing_feature extends express_entity \{

// Ficlds

public String its_id;

public workpiece its_workpiece;

public machining_operation[] its_operations;

// Constructors

public manufacturing_feature() \{\}

public manufacturing_feature(String its_id, workpiece _its_workpiece,

machining_operation []_its_operations) \{\}

$/ /$ Methods

public ArrayList get_prop_names() \{return null:\}

public void set_attributes_from part21_line(String part21line, ArrayList A) \{ \}

public void set_its_id(String_its_id) \{\}

public void set_its_workpiece(workpiece_its_workpiece) \{\}

public void set_its_operations(machining_operation[]_its_operations) \{\}

public String get_its_id() \{return null;\}

public workpiece get_its_workpiece() \{return null;

public machining_operation[] get_its_operations() \{return null:\}

public ArrayList get_attributes() \{ return null; $\}$

public ArrayList get_output() \{ return null; \}

Figure 5.12 A ISO 14649 package for manufacturing features

A sample of code containing the implementation and structure of one the most general class turning feature library is given in figure 5.13 and the details are presented in Appendix C. This code consists of public class, constructors and methods.

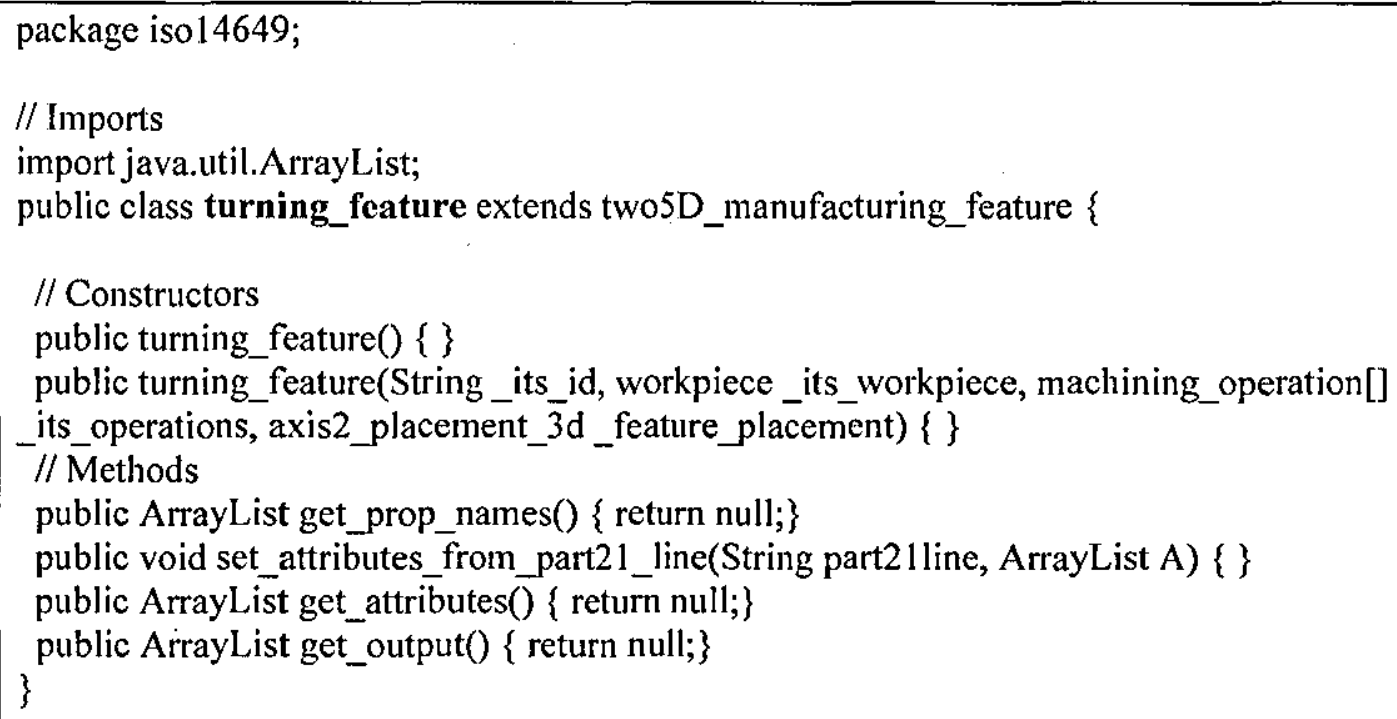

Figure 5.13 Sample code for general class turning feature library 


\subsection{Representation Diagram}

The UML was developed representing the representation and model diagrams, the constraints and the extension mechanisms. UML is the most widely known and used standardized notation for object-oriented analysis and design. The most useful standard UML diagrams are; use case diagram, class diagram, sequence diagram, state chart diagram, activity diagram, component diagram and deployment diagram. For the purposes of this research, only class diagrams and their notation have been used.

In order to develop the system, a set of computing tools will be used. The Java programming language is used for the actual development of software components based on the object oriented methodology and UML is utilised as the modelling language. The manufacturing models refer to the process that deals with production such as operation and strategies. The UML represents the various objects for the SCSTO manufacturing environment and the relationships between these objects. Each data type in these models is based on ISO 14649 part 10 (ISO, 2004a) and part 12 (ISO, 2005a). Development of the graphical user interface (GUI) is based on the literature review and current systems. It starts with gathering the information related to SCSTO, focuses on the product and manufacturing models to provide additional input into the construction of the model and forms the basis of the conceptual information. A comprehensive task description of the proposed system is presented in Chapter 6.

\subsubsection{Turning Features}

Figure 5.14 shows the turning features classes, and consists of outer_round, revolved_feaure and knurl. Each class has other sub-classes as shown in the figure. Any definition of new or existing features will refer to the turning features classes whether that definition is recognised or not. 


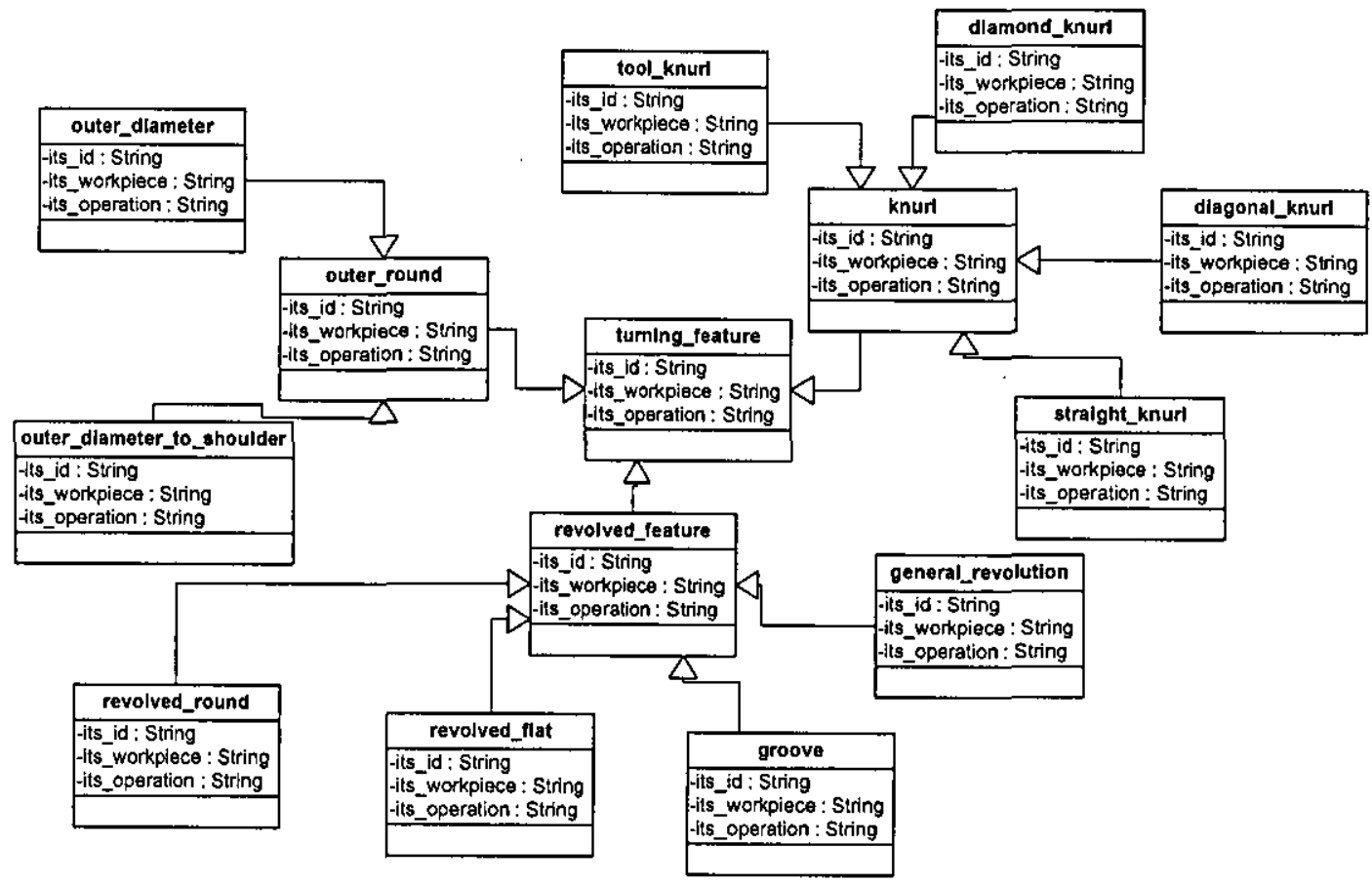

Figure 5.14 UML diagram for the turning feature

The UML diagram of an example, outer_round, is represented by the inherited structure of the classes with properties and methods shown in figure 5.15.

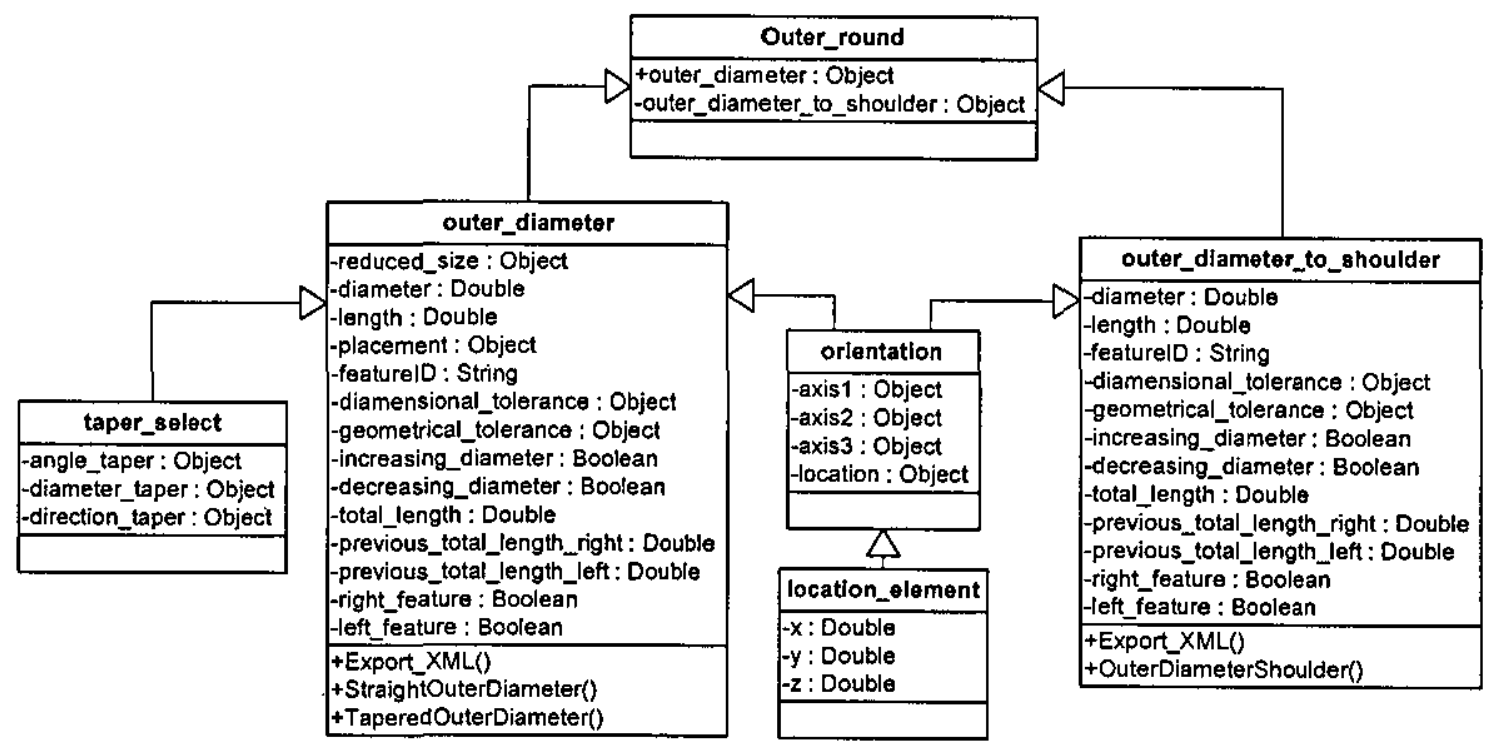

Figure 5.15 UML diagram for the feature outer_round 


\subsubsection{Turning Centre Classification}

In order to consider the turning machine in the proposed prototype system, they have simply been classified according to their axes, from 2 to 8 , plus multiprocess machines. Figure 5.16 shows the detailed classification in a UML diagram with number of axes, the type of construction, horizontal or vertical spindle and the number of work holding devices that the turning centres have and single or twin spindles. This classification also considers the number of tool holding devices as either single or dual turrets. Though this figure is not totally inclusive of all turning centre designs it does illustrate the enormous variety of configurations and complex solutions that can be provided for rotational component manufacture. 


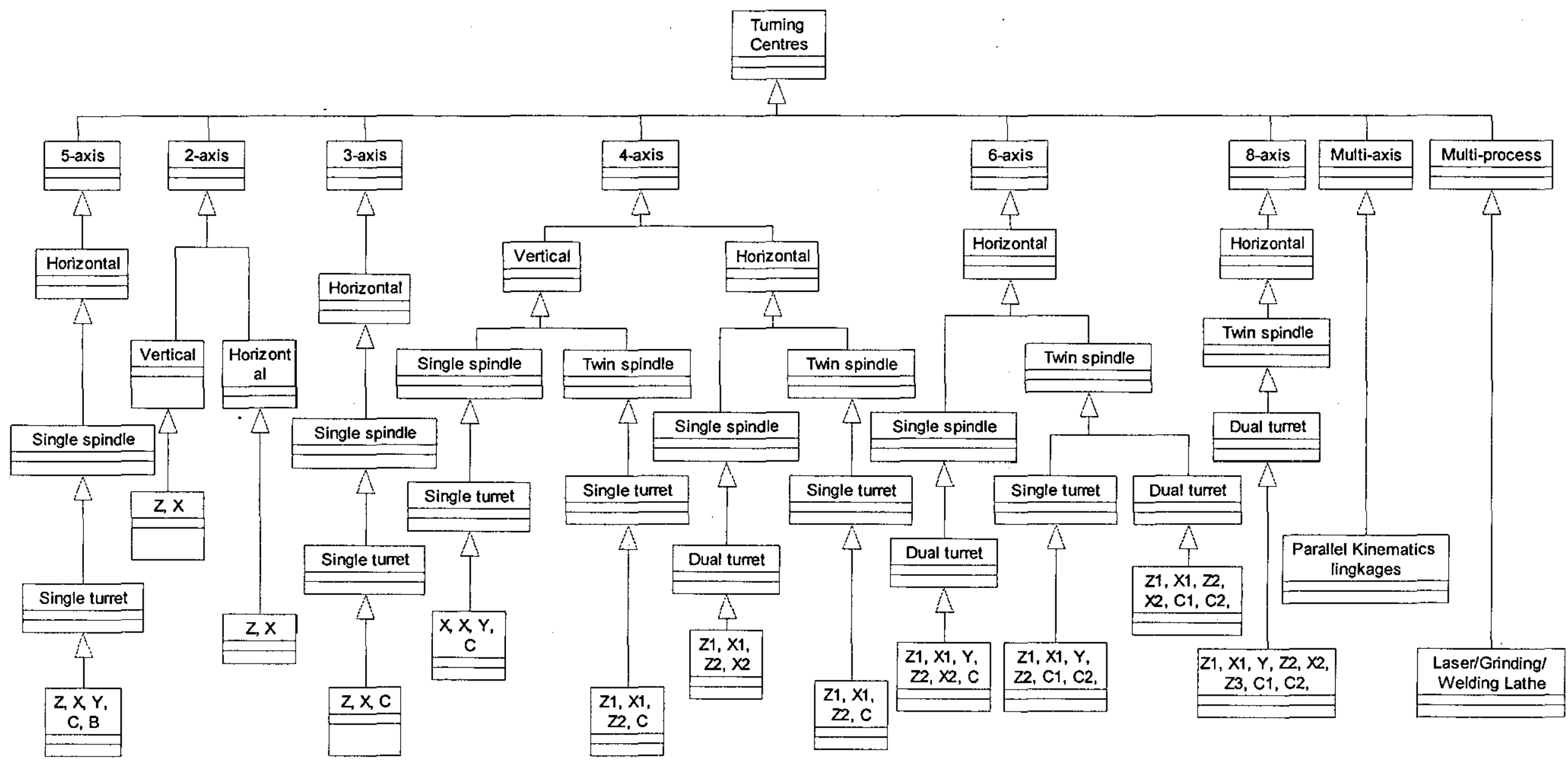

Figure 5.16 Turning centre classification 


\subsubsection{Machining Operations}

The turning machining operation class uses the taxonomy described in ISO 14649 Part 12 (ISO, 2005a) and is divided into the sub-classes facing, contouring, knurling, grooving and threading as shown in figure 5.17. The turning_machining_operation is defined under the machining_operation class defined in ISO 14649 Part 10 (ISO, 2004a) and drilling operations are also part of the milling_machining_operation class defined in ISO 14649 Part 11 (ISO, 2004b). Turning machining operations model the functional capabilities of the turning process to perform the machining operations such as workpiece_setup and turning_machining_operations.

\subsubsection{Manufacturing Strategy}

Manufacturing strategy for turning operations is called turning machine strategy and is defined as part of the manufacturing strategy class. Figure 5.18 shows classes under turning machine strategy such as unidirectional, bidirectional, contour_turning and grooving_startegy. 


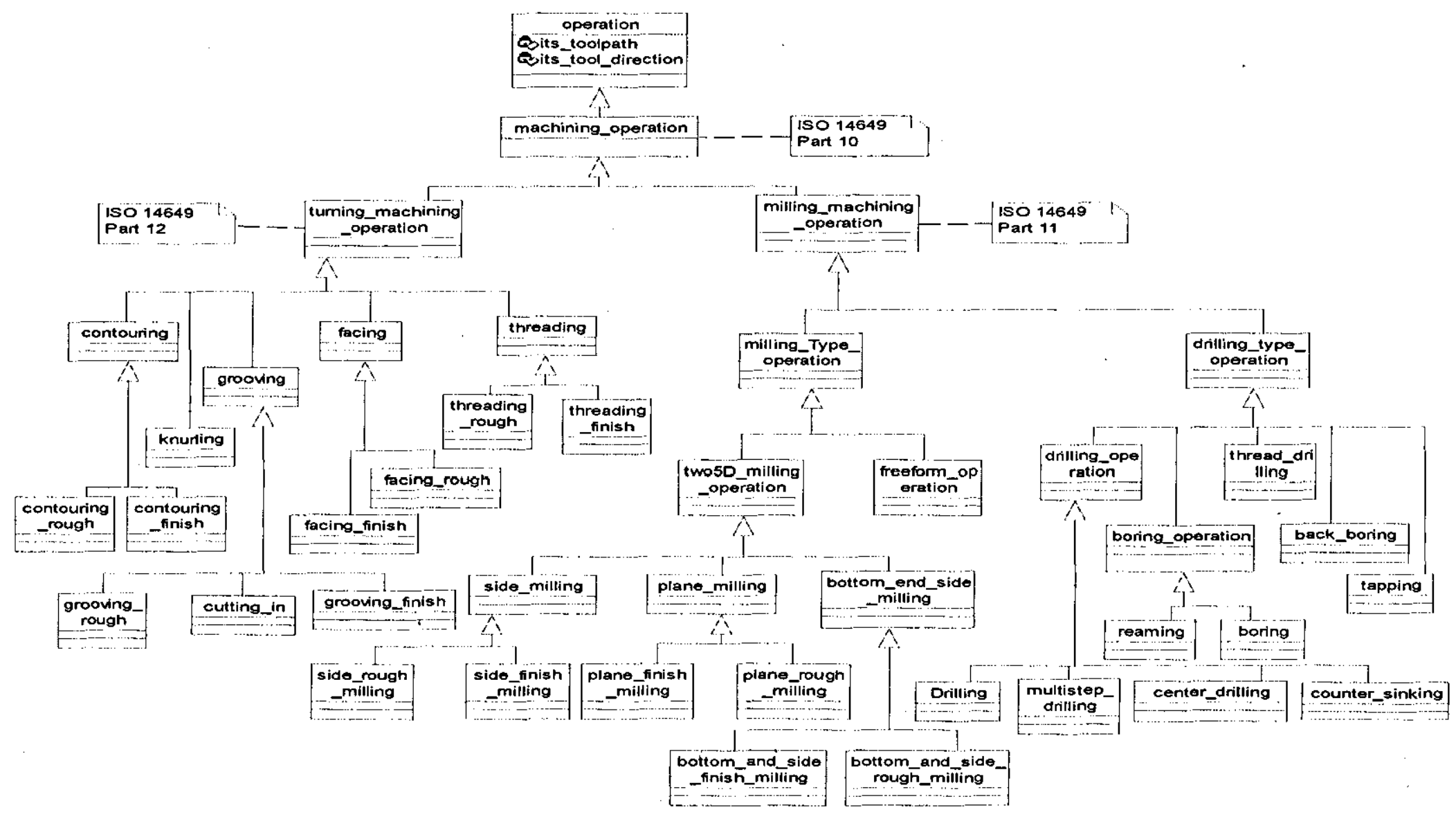

Figure 5.17 Class diagram for Operation 


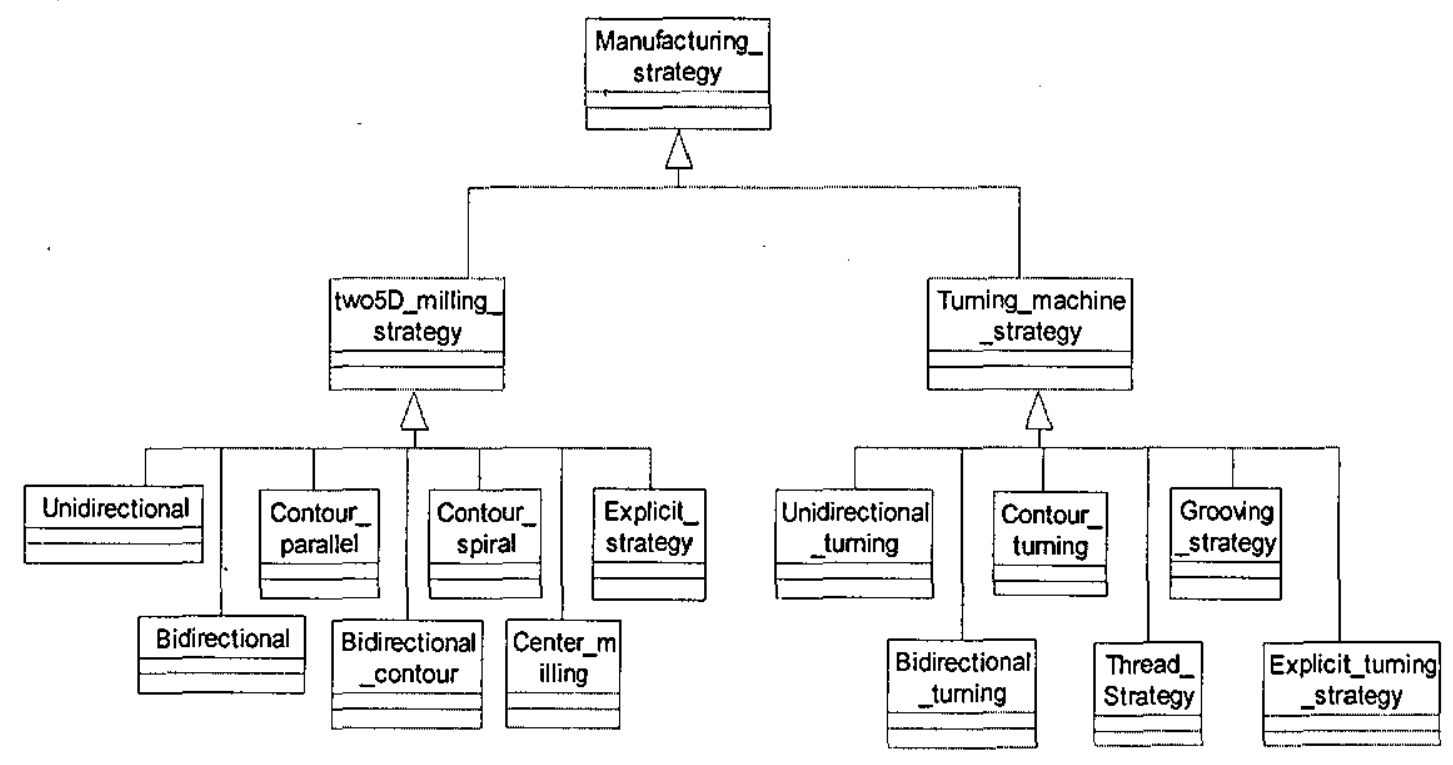

Figure 5.18 Class diagram for manufacturing_strategy for turning operations

\subsubsection{Manufacturing Resources}

Manufacturing resources for turning operations are called turning machine functions, turning cutting tools and turning machine tool bodies defined under machine function, machine tool and tool body. Figure 5.19 shows classes under turning resources such as turning_cutting_tool and turning_machine_tool_body. 


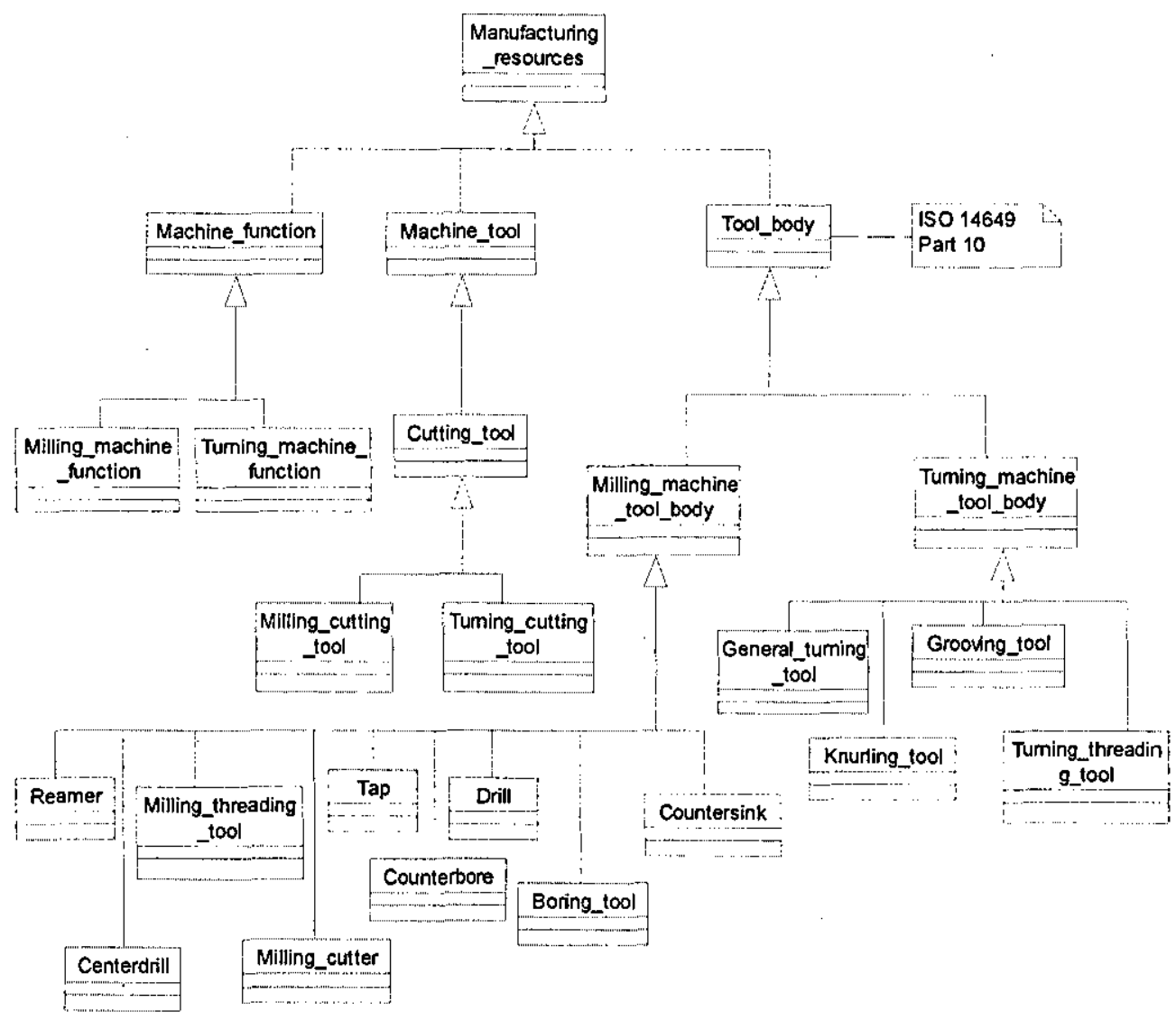

Figure 5.19 Class diagram for Manufacturing_resources

\subsubsection{The UML Diagram for SCSTO}

Finally after considering the product data model and the manufacturing data model referring to manufacturing resources, processes and strategies the author has developed a model for SCSTO using STEP-NC schemas as shown in figure 5.20. This model becomes a platform for developing the SCSTO prototype based on functional and information referring to resources, processes and strategies. 


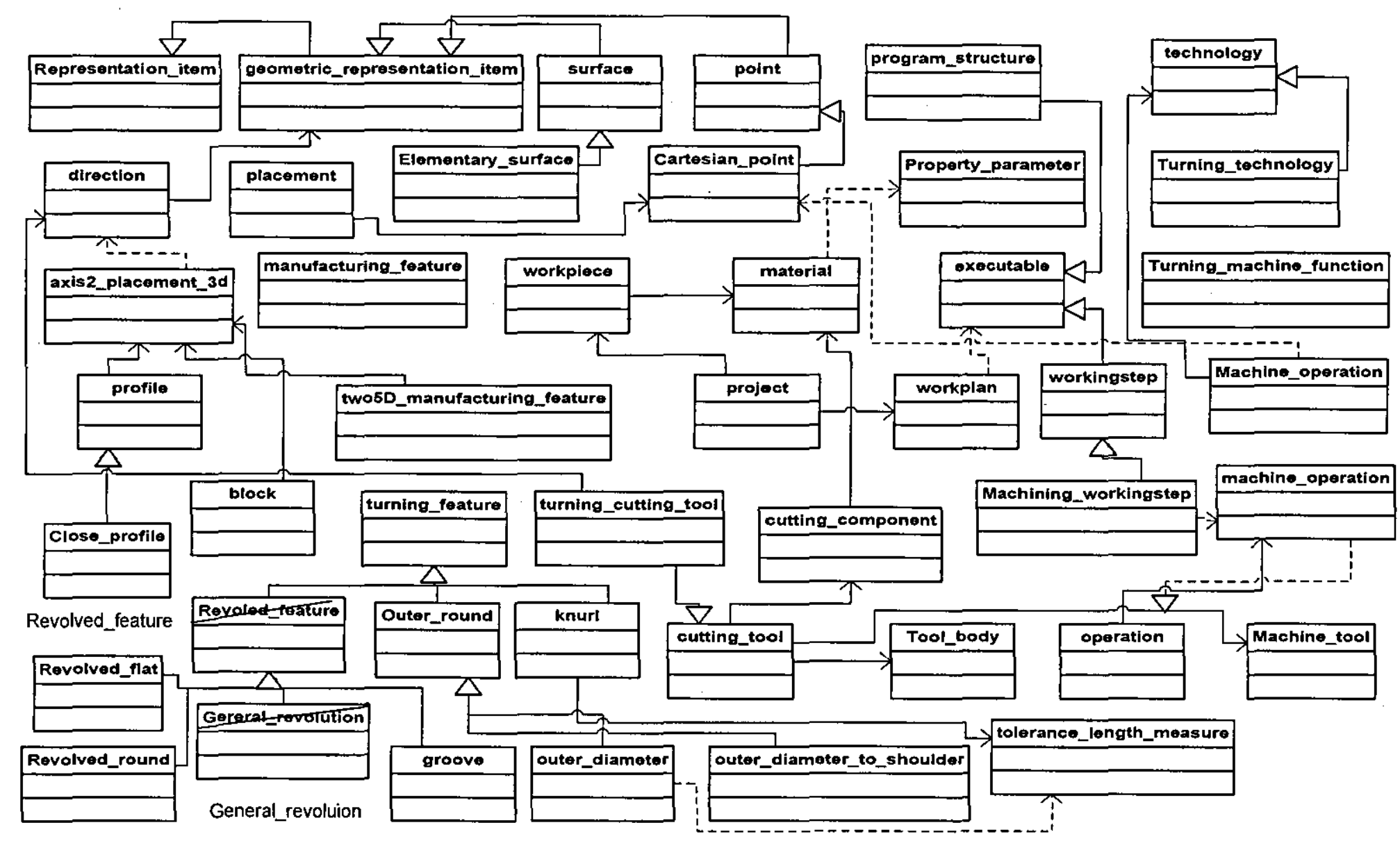

Figure 5.20 UML Diagram for SCSTO 


\subsection{Summary}

This chapter has presented the STEP-compliant information models (UML diagrams) that support the proposed framework for turning operations. The STEPcompliant product information model provides the geometric information of the individual part and its features information. The features in this case are specified according to definitions provided by ISO 14649 for $2 \frac{1}{2} \mathrm{D}$ manufacturing and transition features. These features including turning features such as outer round, knurl and revolved feature.

Beside turning features, the main models also include turning machining operations, manufacturing resources and turning strategies. These information models have been defined through the use of object oriented UML in implementing the prototype system called SCSTO. The information model represents the manufacturing capabilities of the turning operation by implementation of both the Product Model and the Manufacturing Model using the combined methodologies of Integration Definition for Function Modelling (IDEF0) and UML. The following chapter will describe and illustrate the STEP Compliant System for Turning Operation (SCSTO) using a STEP-based machining features approach. 


\section{Chapter 6 - Realisation of a STEP Compliant System for Turning Operations (SCSTO)}

\subsection{Introduction}

This research focuses on the implementation of a new standard adopted for CNC machining and is based on the design and specifications to bring together the information models, tool database, user interface and STEP output file into a prototype system. The functions and outputs of these components and the implementation methods have already been described in Chapter 4. This chapter describes and illustrates the STEP Compliant System for Turning Operations (SCSTO) using a STEP-based machining features approach. The chapter is divided into two parts; i) Development of SCSTO and ii) Application of SCSTO. The system was developed as an object-oriented feature-based design system to support concurrent design and to generate process plans. This approach was chosen since it represents a logical means for representing real world objects like machine components (Chep and Tricarico, 1999).

As mentioned in the research specification, the information can either be about the product to be manufactured (product model), or the manufacture of the product (manufacturing model). The information models described in the previous chapter support the proposed system together with the information models defined in the ISO 14649 standard used to create the NC program (ISO, 2003b). This is followed in this chapter by detailed elaboration of the system development. The implementation of SCSTO was conducted in three stages. The first stage involved developing the information model using UML diagrams. The second stage concentrated on the development of the prototype based on the model proposed and 
the third stage is the evaluation carried out by implementation of two case study components that will be discussed in Chapter 7 .

\subsection{Assumptions}

The proposed model for SCSTO is based on two assumptions:

i. Data gathered from the literature review on STEP compliance is sufficient as the basis for the modelling of SCSTO.

ii. ISO 14649 Parts 12 and 121 are core references for STEP compliance for turning operations, but some aspects were still under discussion during this research.

\subsection{Overview of SCSTO}

The overall system as shown in figure 6.1 consists of three main subsystems; manufacturing features creator, manufacturing operations and program generator. In SCSTO, the user has a choice to either create a new project with new features (refer to figure 6.1 (1) and figure 6.1(2)) or open a project from a CAD file in STEP AP 203 format (refer to figure 6.1 (2) and figure 6.2). Geometry described in the AP 203 format defines the features but not their location and orientation. Feature geometry is defined in ISO 14649 Part 12 (ISO, 2005a) and has been described in section 4.7 and more formally described in terms of UML diagrams in section 5.9. The placement and location of the feature needs to be provided by the user once the feature has been extracted from the AP 203 file.

The alternative is to create the feature by user definition which is limited to features within the feature library. If the user needs to define a new feature, the first step in designing a part is to specify the base part shape and the dimensions associated with the shape to define its size. In this thesis the base part shape is limited to cylindrical, so only this shape can recognised for further processing. The base part shape is considered as the initial shape of the material before machining the features. 
Chapter 6

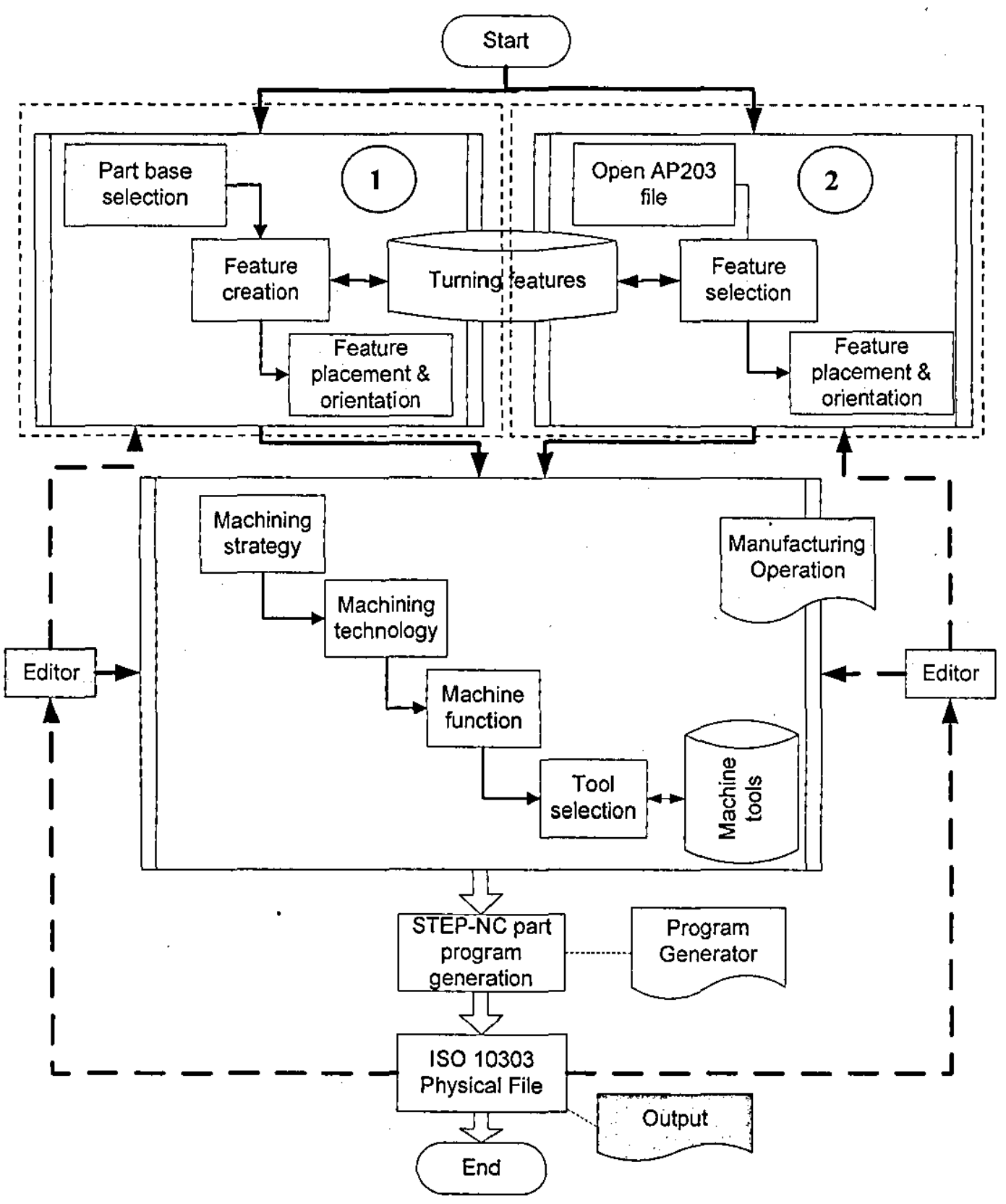

Figure 6.1 Architecture of SCSTO

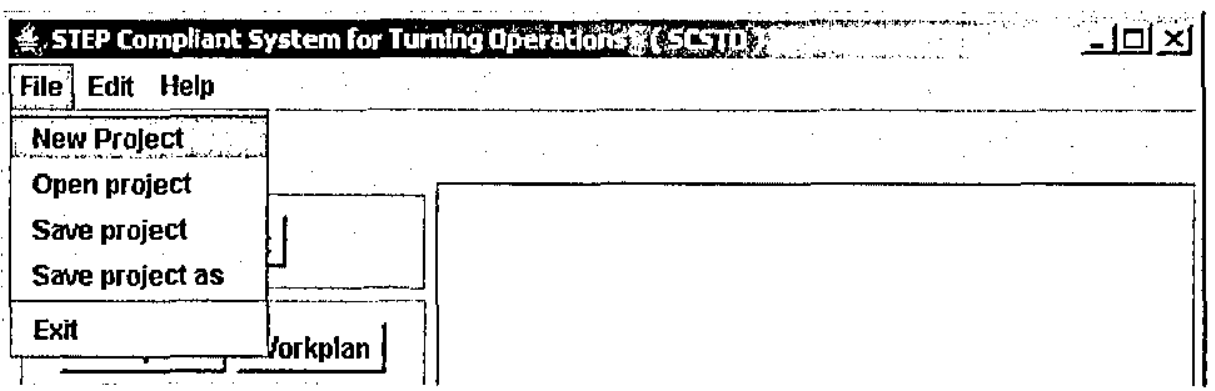

Figure 6.2 Creation of a new project menu 
Cylinder length and diameter are the only parameters needed to define the base part that is positioned with the z-axis parallel to the longitudinal axis of the shape. The $x$ and $y$-axes are orthogonal to the z-axis. The axis origin is positioned at the centre of the circular profile forming the bottom of the cylindrical base part.

The second subsystem consists of five major components:

i. Integration and preparation of part design data.

ii. Selection of machining strategy

iii. Selection of machining technology.

iv. Selection of machine functions.

v. Selection of cutting tools.

The third subsystem is the Generation of Process Plans as described in section 4.11 to generate a physical STEP-NC process plan file. This file consists of information such as machining operations, cutting tools, machining parameters, etc. If the user is not satisfied with this part program, it can be edited to modify either turning features or turning operations.

The turning machining concept is based on 2D profiling except for drilling perpendicular to the $\mathrm{z}$ axis. Figure 6.3 shows the raw material and the working area which is defined as the Boolean difference between the raw material and the finished part. 

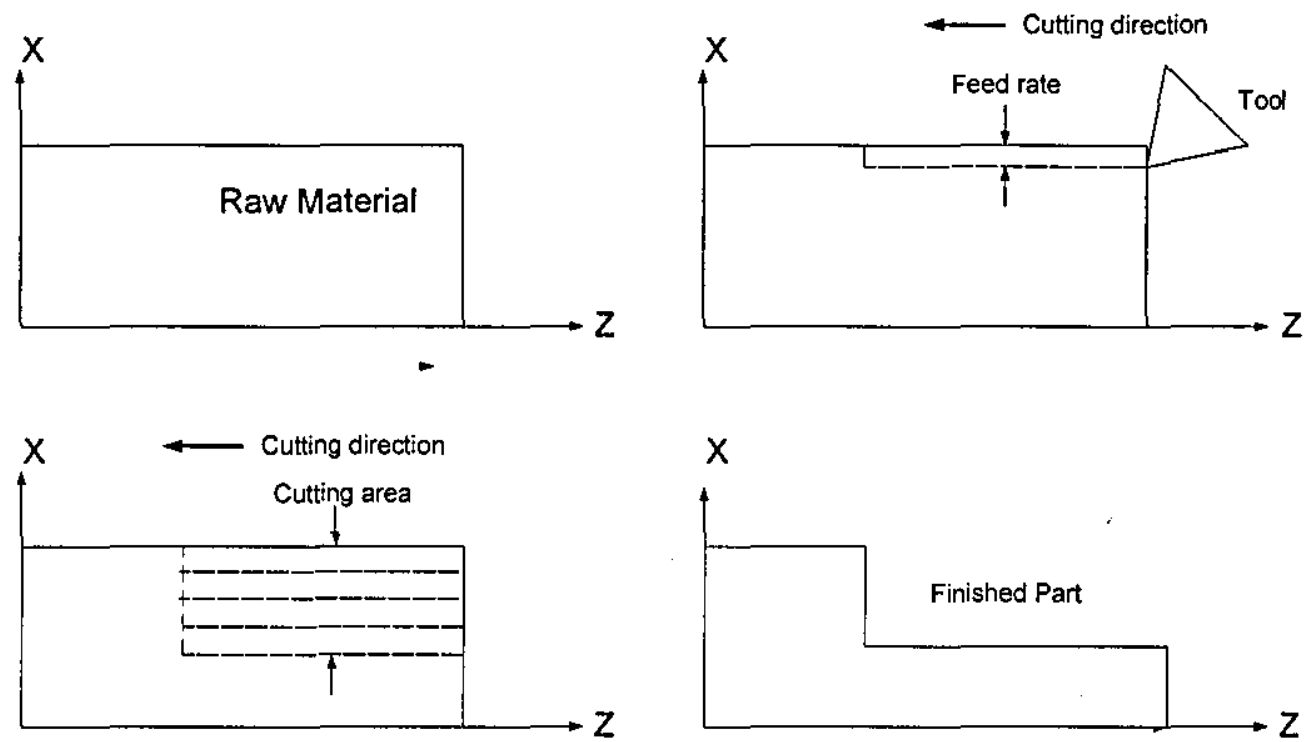

Figure 6.32D turning machining concept

\subsection{SCSTO Tools}

There is a large variety of software development tools available on the market. This implies that it should be possible to choose the most suitable tool for a specific purpose. However, sometimes the effort involved in learning to use a new tool, just for one case, might be greater than the benefit. On the other hand, choosing the wrong tool has resulted in very discouraging experiences and reluctance for the development of prototypes in general. After consideration of the tools, costs, flexibility and recommendations from previous researchers, the author used the tools as shown in table 6.1 for SCSTO development. 
Table 6.1: SCSTO development tools

\begin{tabular}{|c|c|c|c|}
\hline No & Stage & Function & Tools \\
\hline 1. & $\begin{array}{l}\text { Developing } \\
\text { information model } \\
\text { (Discussed in } \\
\text { chapter 5) }\end{array}$ & $\begin{array}{l}\text { Gathering the information related to } \\
\text { SCSTO, with a focus on the product } \\
\text { and manufacturing models to provide } \\
\text { additional input into the construction } \\
\text { of the model and forming the basis of } \\
\text { the conceptual comprehensive task } \\
\text { description of the proposed system. } \\
\text { Design models were constructed using } \\
\text { UML, since SCSTO is implemented } \\
\text { on an object oriented platform. }\end{array}$ & $\begin{array}{l}\text { UML, Rational } \\
\text { Rose } 2000 \\
\text { (Quatrany, } \\
\text { 1998). }\end{array}$ \\
\hline 2. & $\begin{array}{l}\text { Development of } \\
\text { the prototype } \\
\text { (Design the GUI) }\end{array}$ & $\begin{array}{l}\text { To develop the graphical user } \\
\text { interface (GUI) based on the literature } \\
\text { review and previous systems. It starts } \\
\text { from programming, running and } \\
\text { executing the system. Use of JBuilder } \\
\text { to compile and run Java programs. }\end{array}$ & $\begin{array}{l}\text { JBuilder } 2005 \\
\text { (Landy et al., } \\
\text { 2003). }\end{array}$ \\
\hline 3. & Tool database & $\begin{array}{l}\text { To support SCSTO in tool selection } \\
\text { for machining the component. }\end{array}$ & $\begin{array}{l}\text { JStoreData } \\
\text { (Borland, 1997) }\end{array}$ \\
\hline
\end{tabular}

\subsubsection{Programming with JBuilder 2005}

The are many java integrated development environments (IDEs) available today, such as Borland Jbuilder, IBM Eclipse IDE, Oracle JDeveloper, Xinox JCreator, IntelliJ IDEA, NetBeans IDE, GNU Java IDE, Sun Java Studio Standard 5, Microsoft Visual $\mathrm{J}++$, etc (Nguyen and Abdali, 2005). From the three Borland JBuilder 2005 editions (foundation, developer and enterprise), the JBuilder 
Foundation has been chosen because it has a good java development environment for users who want to learn java (Landy et al., 2003) ( for details refer to http://info.borland.com/techpubs/jbuilder/).

\subsubsection{Creating ISO 14649 Classes}

The product model is contained within a set of interconnected objects. The class definition of these objects is based on the entity definitions that exist within the ISO 14649 standard. From the standard, classes are written in the java language as shown in figure 6.4 and an example of the structure of these classes is shown in figure 6.5.

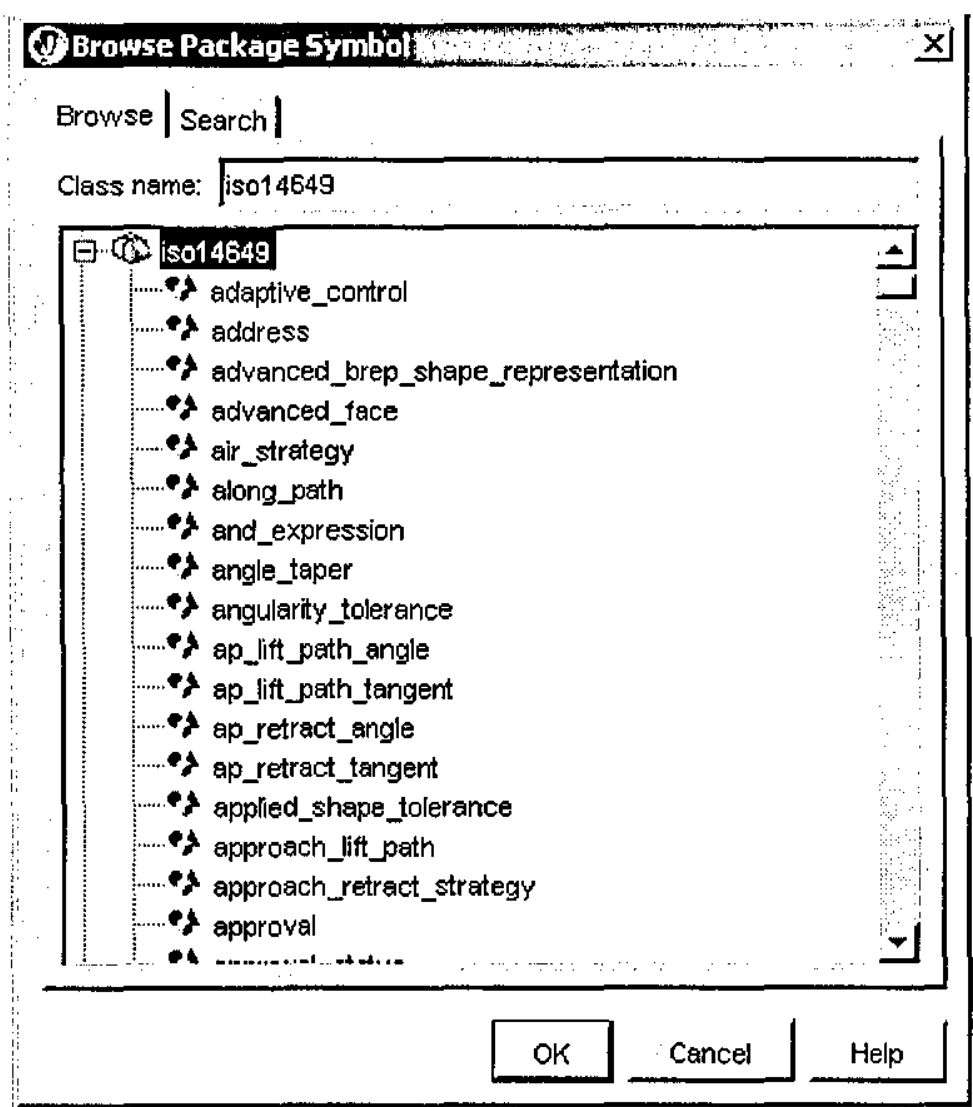

Figure 6.4 Java classes based on ISO 14649 standard 


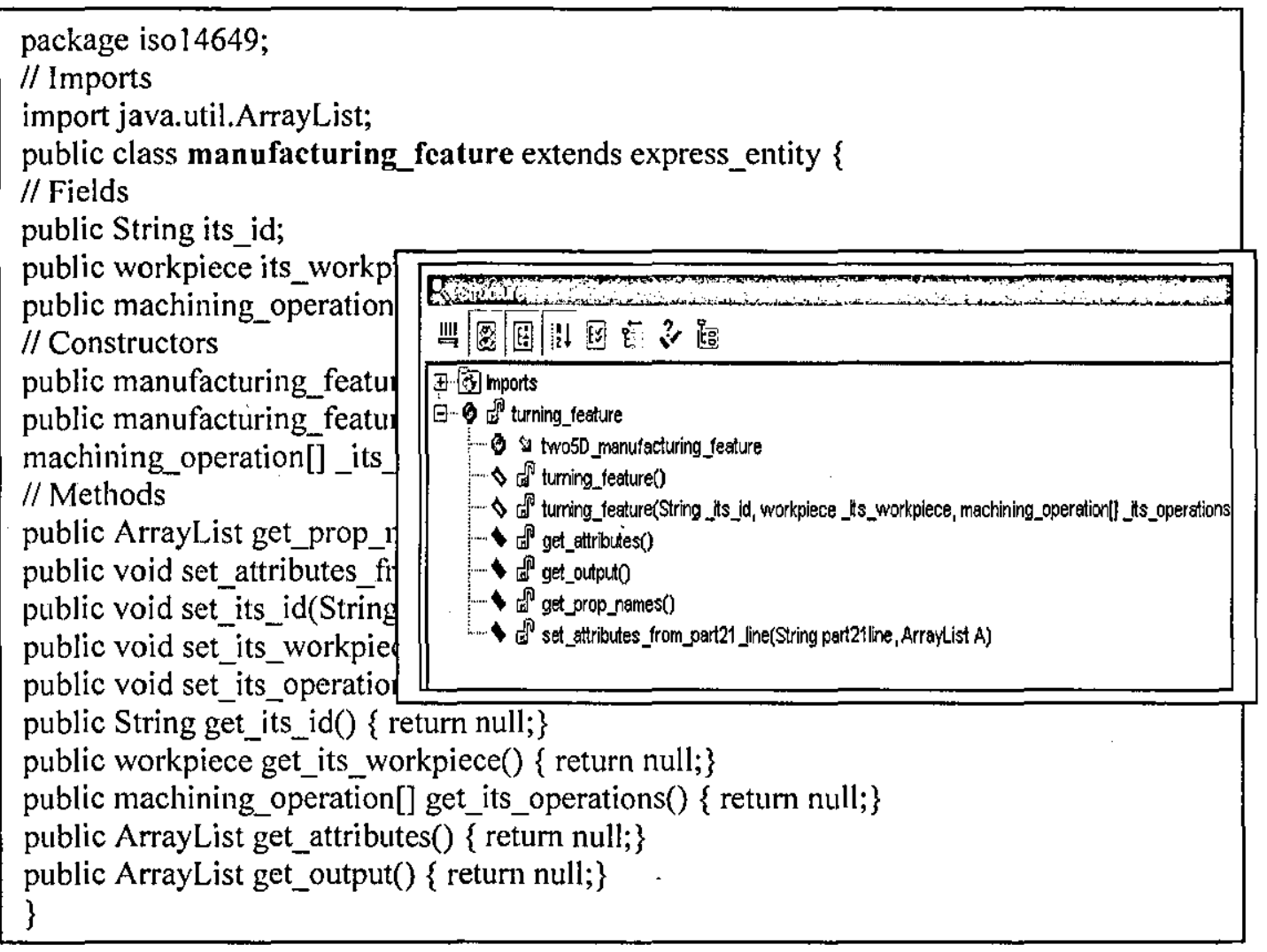

Figure 6.5 Java class structure

\subsubsection{Key Elements of JBuilder}

Before developing a prototype system, it is important to understand the key elements of JBuilder. Figure 6.6 illustrates the screen designer that is used to create a prototype system for turning operations using JBuilder. The JBuilder Screen Designer uses a powerful feature called a two-way designer that allows user interfaces to be constructed either by using the Screen Designer or coding events interchangeably. This approach makes it flexible to use whatever technique works best; depending on what needs to be accomplished. For example, it might be easier to make changes to the code to change properties based on variables. 


\section{a) Screen designer}

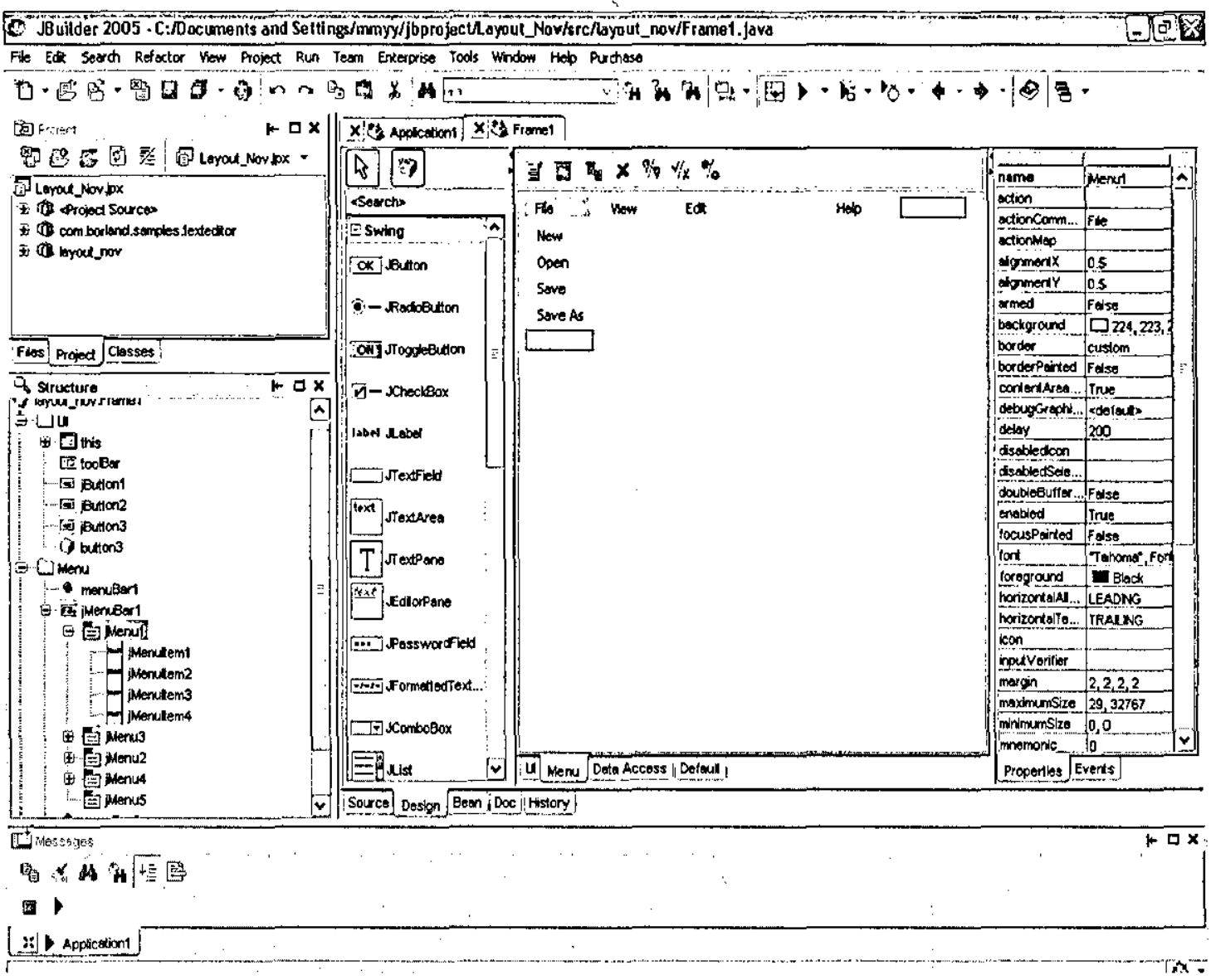

Figure 6.6 Screen designer

\section{b) Common standard control}

The development of the prototype followed the general procedure for developing a java language program and consisted of the creation of a coordinated set of classes and features using standard GUI features such as Pushbutton.

\section{c) Coding events}

Event code is executed upon a given associated action. This is programming based on the event-driven programming model. In other words, code is executed as a reaction to a given event or events. This code is written within an event-handling stub to allow for better organization of code. JBuilder then manages the relationship or the attachment of these code snippets to the corresponding component's event. 
Using the Events tab of the Inspector, the designer can easily attach a new or modify an existing event handler. Figure 6.7, shows the code generated by JBuilder as seen by the designer. For full details refer to the Jbuilder developer guide (JBuilder, 2005). The Code Generated responds to an actionPerformed Event

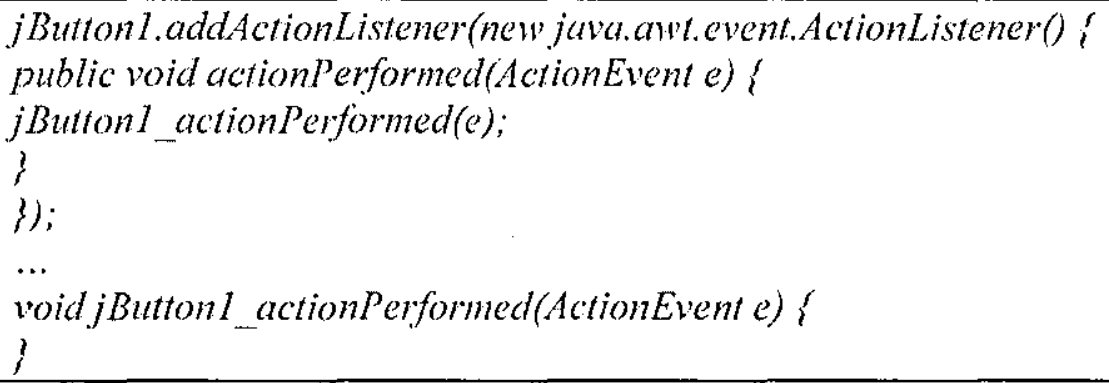

Figure 6.7 code generated by JBuilder

\subsection{System Architecture}

To ensure that the desired features and objectives of the prototype are accomplished, the system architecture for the prototype, illustrated in figure 6.8, was developed. There are four main components that provide the means for developing a STEP compliant system for turning operations. These components are;

i. Information model

ii. Tool database

iii. GUI

iv. STEP File 
SCSTO Architecture

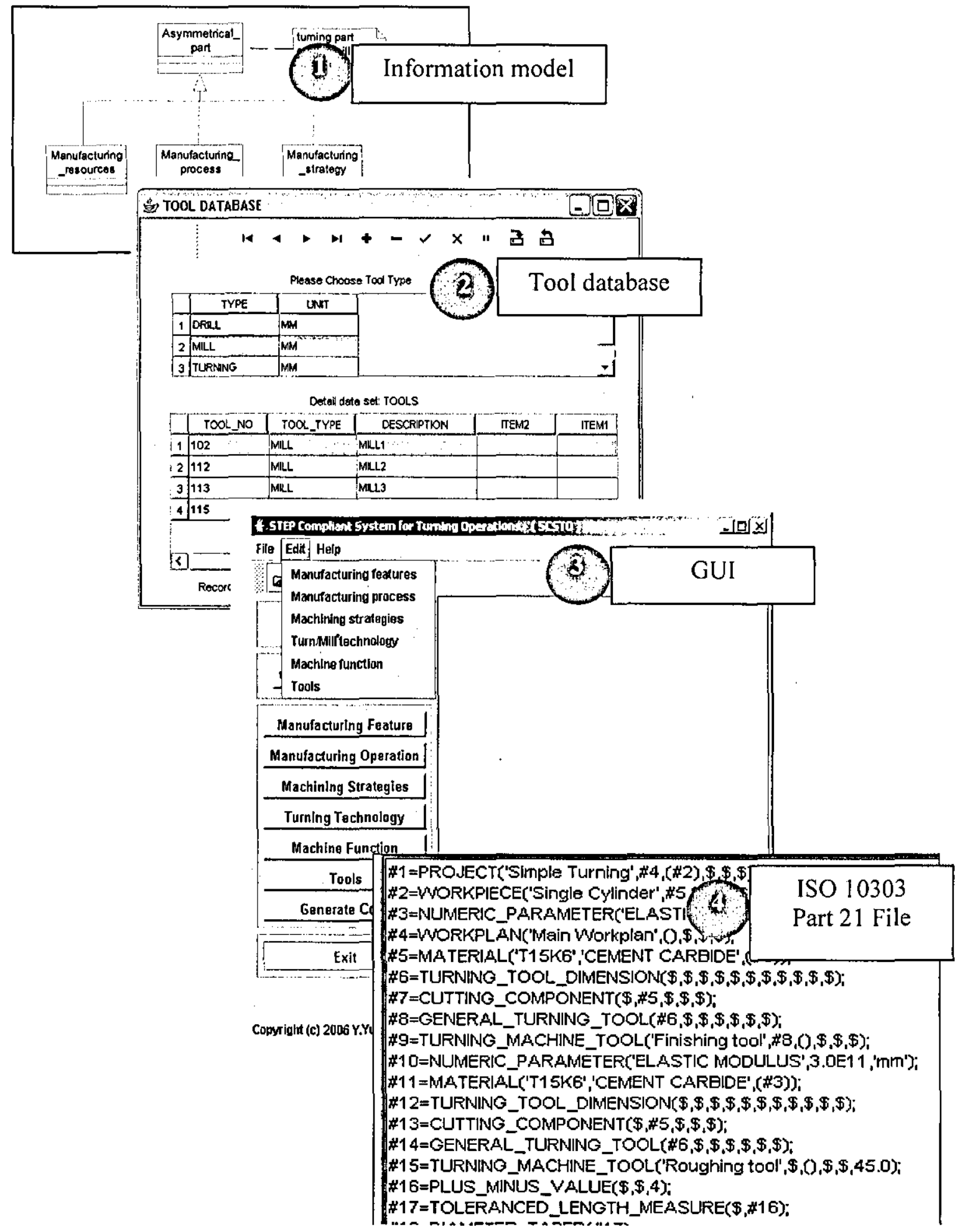

Figure 6.8 The overall framework of SCSTO 
The information model has been discussed in Chapter 5 and the graphical user interface including the tool database will be presented in the following section. A platform called Integrated Platform for Process Planning and Control (IP3AC) has been developed and used by Nassehi (Nassehi et al., 2006b) and was based on the STEP-NC (ISO 14649) data models. IP3AC is essentially a regulated object space where object sets describing manufacturing processes, resources and product geometry can be manipulated. That part of IP3AC that relates to turning operations has been adapted for the work described here.

The classes in the current version of the IP3AC platform are based on the data structures defined within the ISO 14649 (STEP-NC) standard. The Java programming language was used to implement IP3AC. As most of the classes were based on the EXPRESS entities defined by the ISO 14649 standard, it was decided that a code generation program converting EXPRESS definitions to java class code should create most of the classes automatically. An IP3AC executable .jar file has been added under libraries (project properties) as shown in figure 6.9.

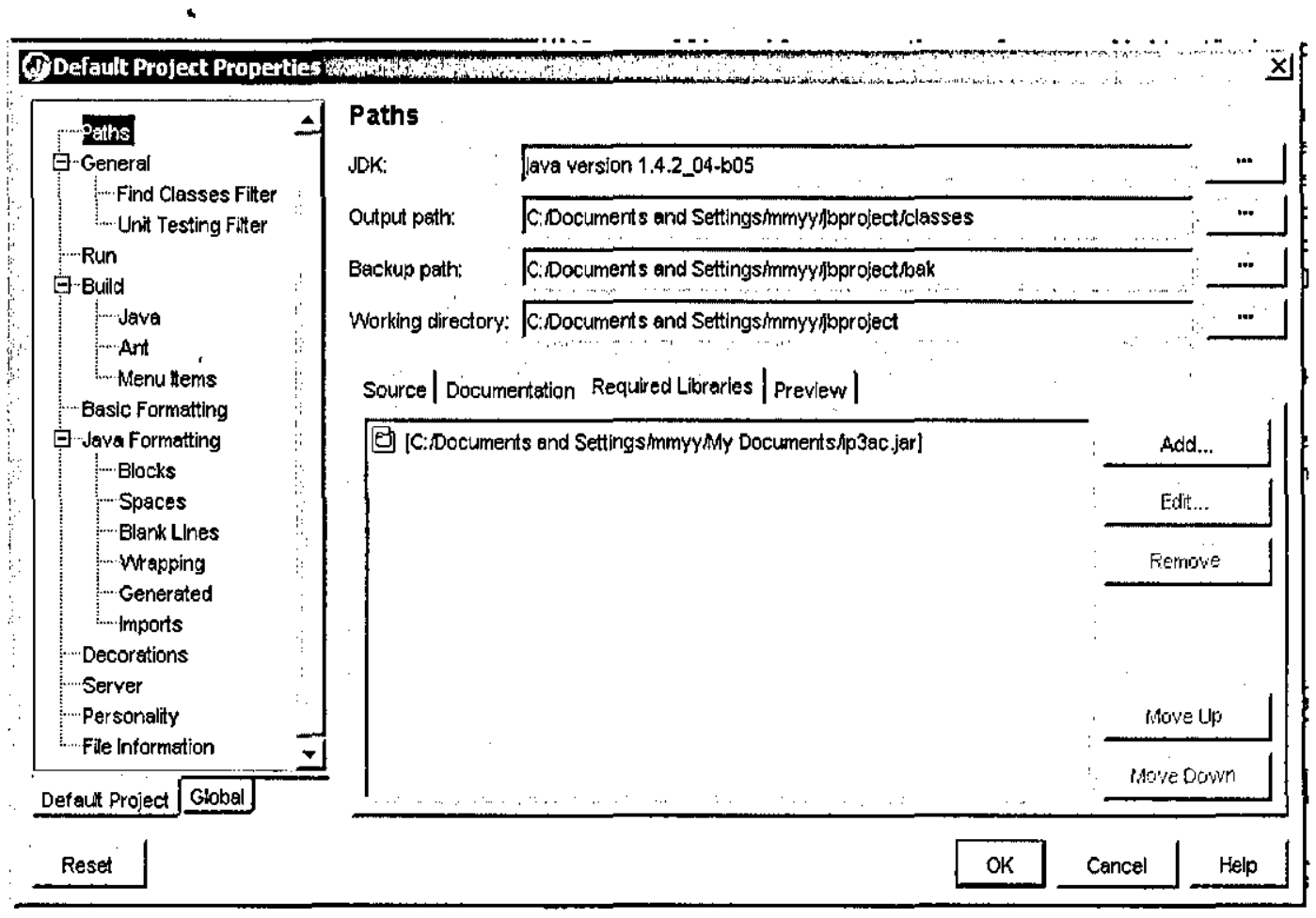

Figure 6.9 The IP3AC project properties 


\subsection{System Development}

Running SCSTO is the same as any Windows-based program. When SCSTO starts, the starting welcome window is displayed (Figure 6.10). The Next button goes to the main window frame and provides the user with several options to start the modelling process. From this window the user can start with;

- Create a new project.

- Open an existing project.

- Edit an existing project

- View SCSTO Help.

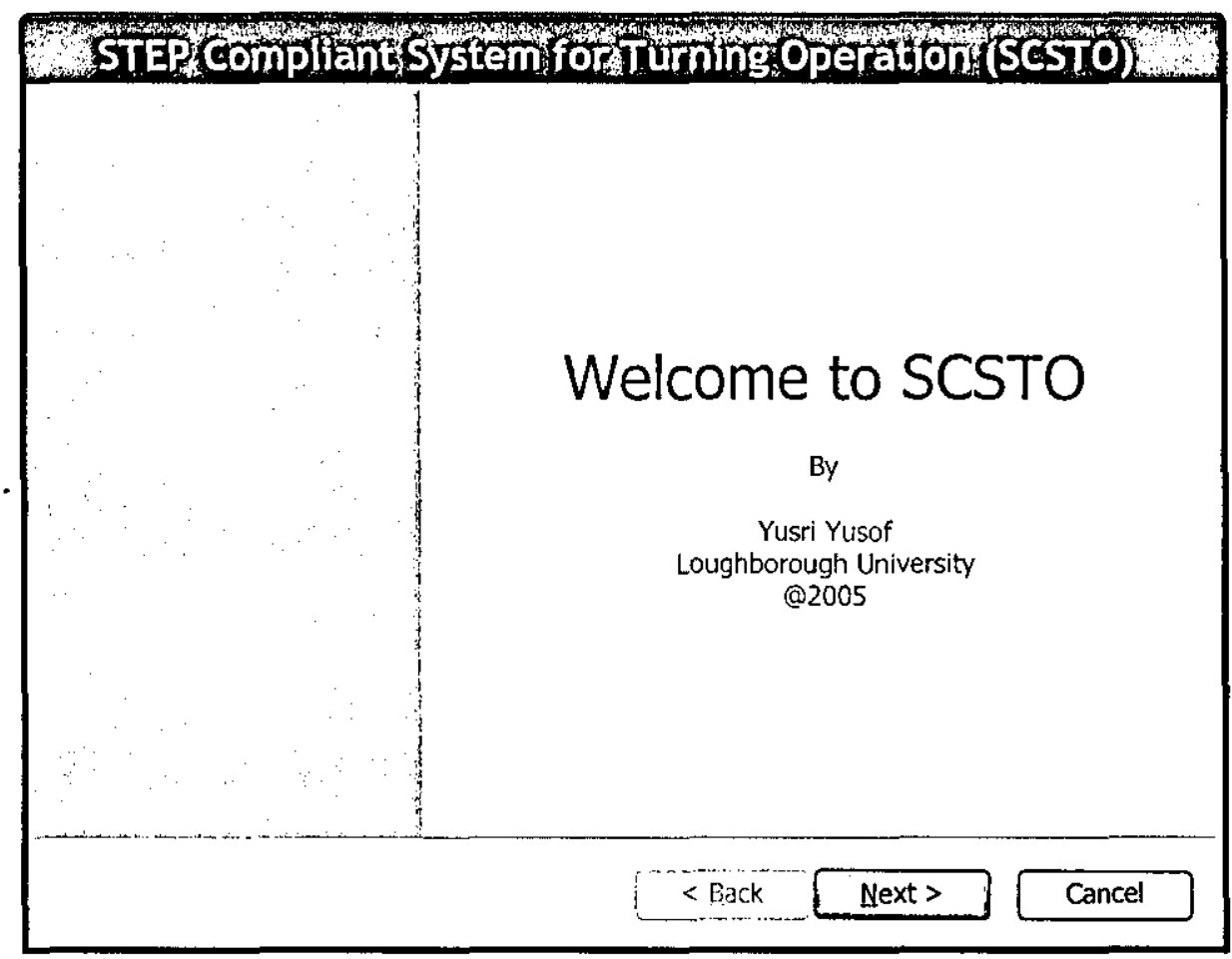

Figure 6.10 SCSTO welcome displayed

\subsubsection{User Interface}

The SCSTO system adheres to the Windows standard for user interface design. All functions can be accessed from the pull-down menus, and common functions are accessible via toolbar icons. Figure 6.11 shows the SCSTO user 
interface window. It consists of several pull-down menus and toolbars. Figure 6.12 shows an example activity diagram for a new feature. Detailed activity diagrams for SCSTO are presented in Appendix D.

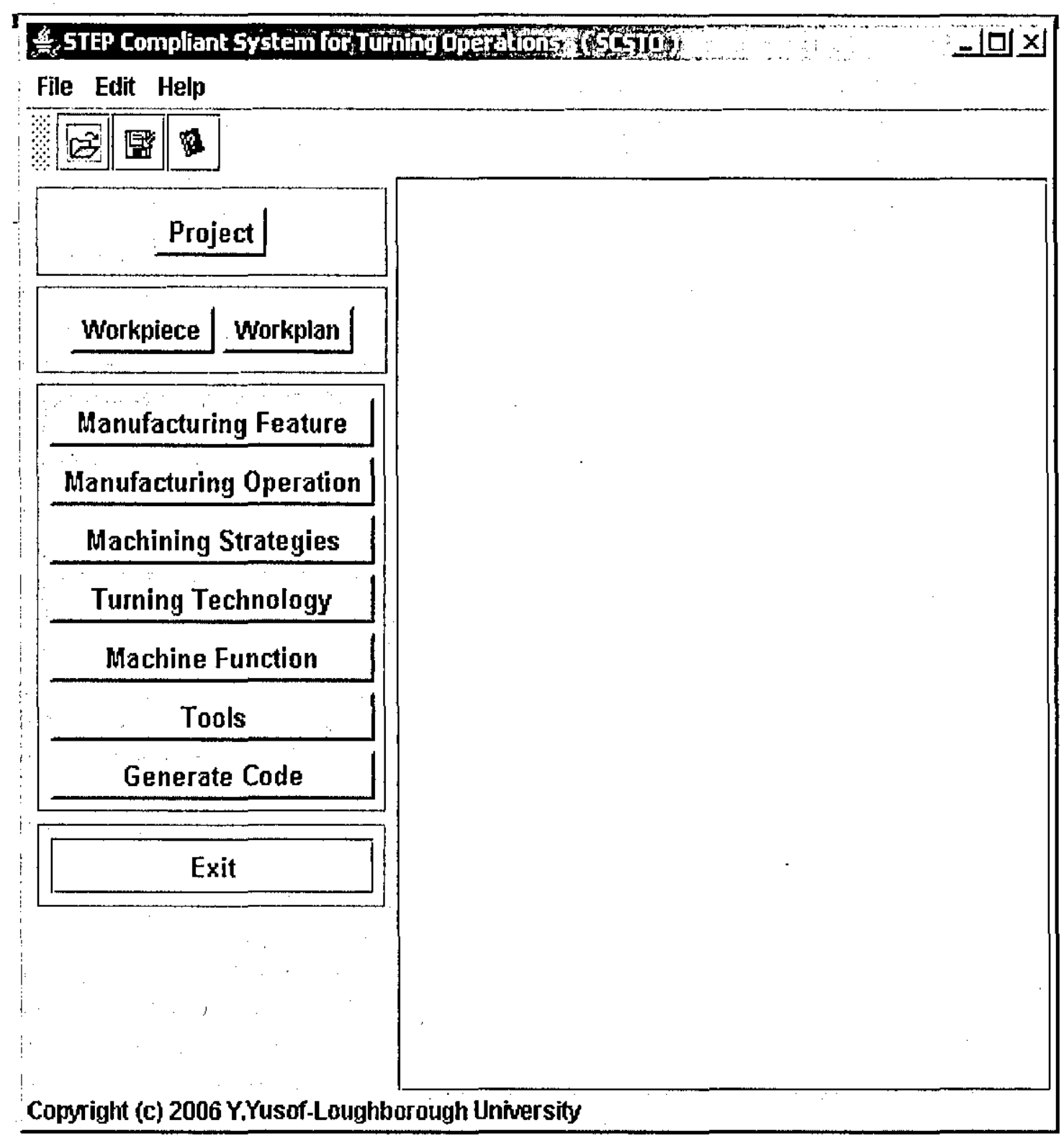

Figure 6.11 shows the SCSTO user interface window. 
Chapter 6

Activities Diagram - New Feature

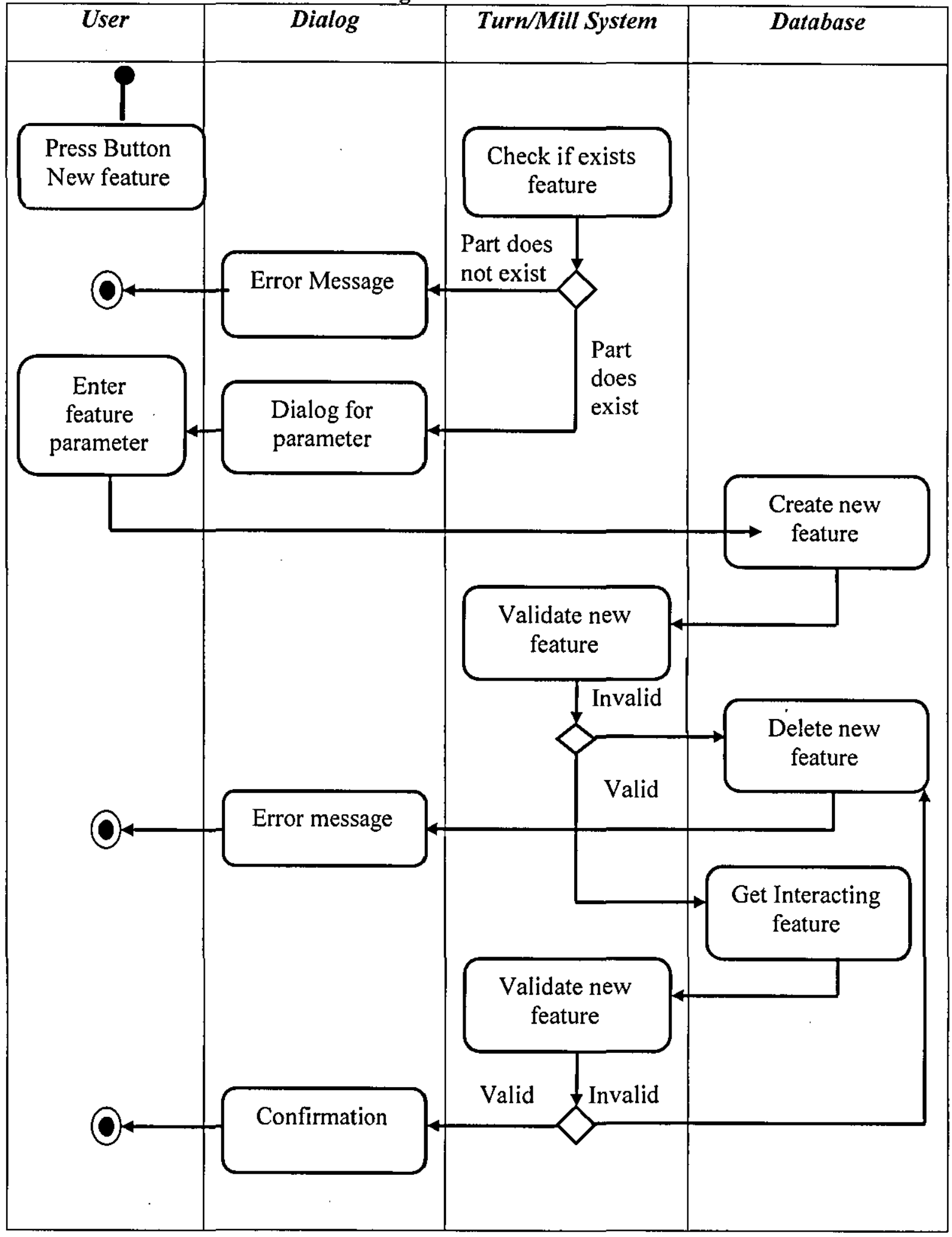

Figure 6.12 Example activity diagram for new feature 
Figure 6.13 , shows the project window which has project, workplan and workpiece fields. This information will appear at the top of the output file. Each part programme, i.e. data model, based on ISO14649, must include exactly one toplevel entity called project. The project indicates the workplan to be executed upon interpretation of this model, as several workplans might be included, and it may also provide the workpiece upon which actions are to be performed (ISO, 2004a)

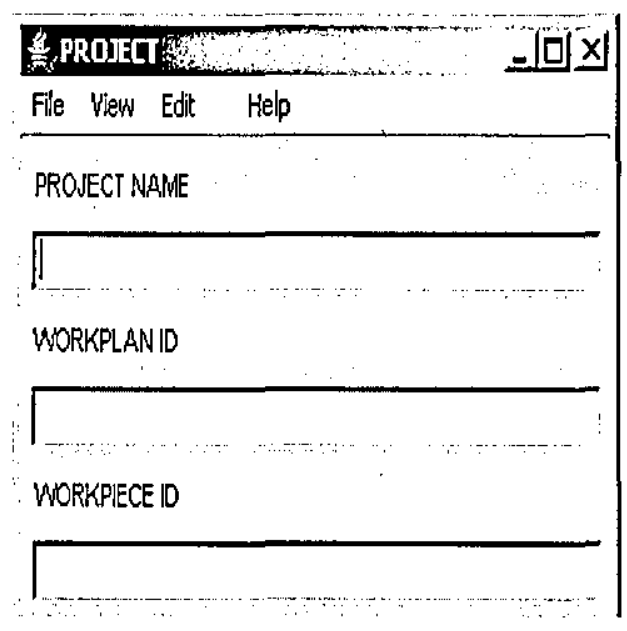

Figure 6.13 Project dialog.

\subsubsection{Feature Selection}

SCSTO is based on STEP feature-based design and it defines part data in terms of machining features and their technical attributes based on STEP-224. After creating the base part, the feature attachment processes can be started. The designer can start with any feature depending on his/her design for manufacturing intent. Feature selection is done by clicking "manufacturing feature" button and a pop up window will be displayed as shown in figure 6.14. As mentioned in section 5.8, all the turning features are stored in the turning feature library and each is from ISO 14649 Part 12 and based on ISO 10303 AP 224. After features have been selected, detailed data such as dimensions and locations need to be defined by the user. The system also needs to know which setup the feature needs to be located in. 
As soon as the feature has been selected, a feature insertion point is specified as a location for attachment to the part base. After that has been completed, a Boolean operation is performed at that intersection, either an addition or subtraction depending on the manufacturing type selected. This process needs be done after all the features have been created. Having performed the Boolean operation, this step and part creation phase is complete.

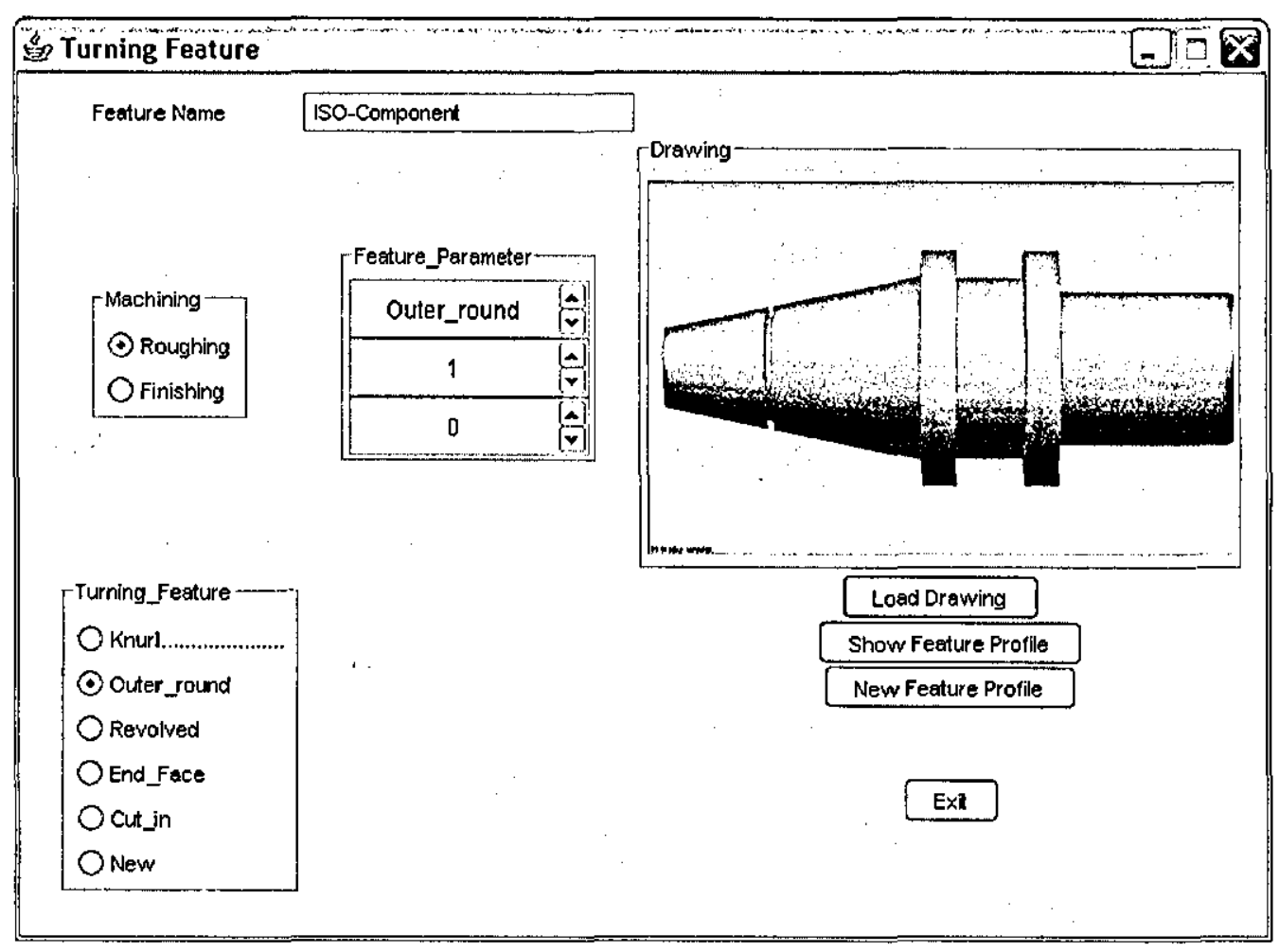

Figure 6.14 Turning feature selection

\subsubsection{Turning Machining Operations}

One of the major functions of the proposed system is machining operations, to manufacture the part. As mentioned in section 4.6, generally, there are two types of machining operations: roughing and finishing. Roughing is used to remove material from the original raw material by multiple surface passes down to the finishing allowance. Finishing then removes the finishing allowance to yield the final form of the feature. The operation is one of the following: facing, grooving, 
contouring, threading, or knurling and for milling, drilling, boring, centre drilling and reaming. Due to special machining the proposed system covers all types of turning machining and only drilling under milling operations as defined in ISO 14649 Part 11 (ISO, 2004b). The drilling operations have been combined together with turning operations as shown in figure 6.15 (java programming) and 6.16 (UML diagram).

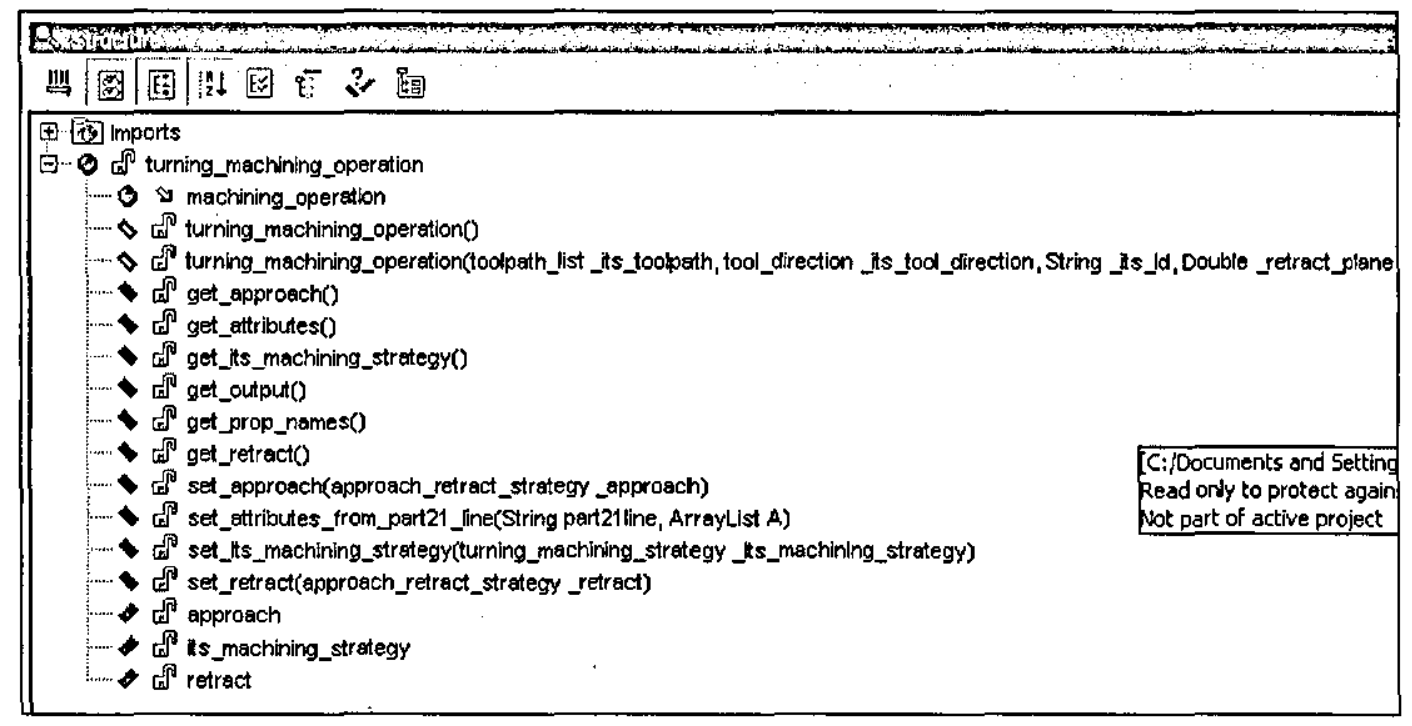

Figure 6.15 Turning machining operation 


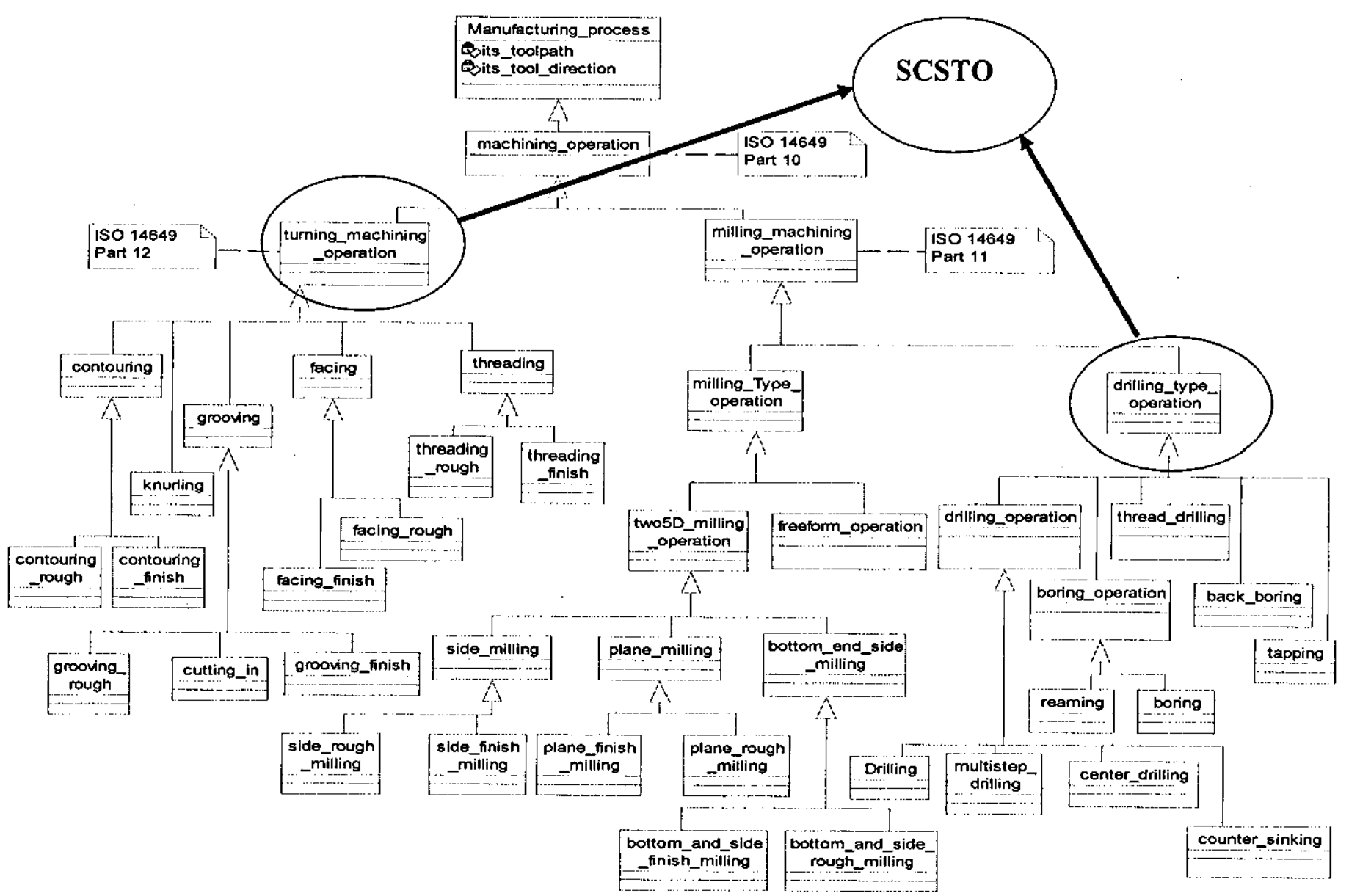

Figure 6.16 Machining operation in UML diagram 


\subsubsection{Tool Database}

A database management system (DBMS) is a system or software designed to manage a database and run operations on the data requested by numerous clients. In the case of SCSTO, the tool database system has been designed to support the easy storage and retrieval of the information and knowledge captured from turning and milling tools. The tool database for SCSTO as shown in figure 6.17 was developed by various operations as shown in figure 6.18 .

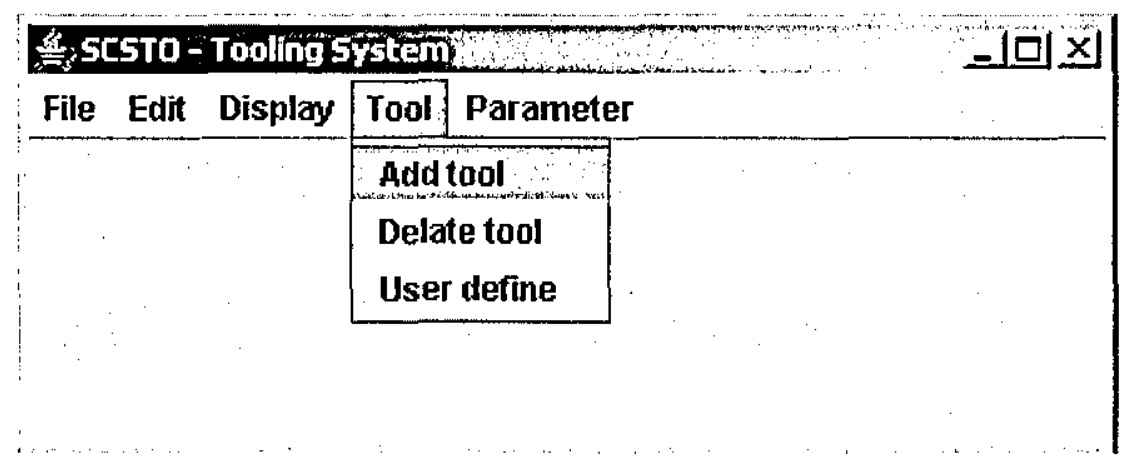

Figure 6.17 SCSTO tooling system

The tool database used in the SCSTO system is based on the STEP-NC standard (ISO14649 Part 121). Referring to ISO 14649 Part 111 (ISO/FDIS, 2004), drilling is put under tooling for milling, so drilling tools are also described. Finally both ISO 14649 document Parts 111 and 121 need to be considered and the database was divided based on turning, milling and drilling tools. Figure 6.19 shows example cutting tool data for turning operations. 
Chapter 6

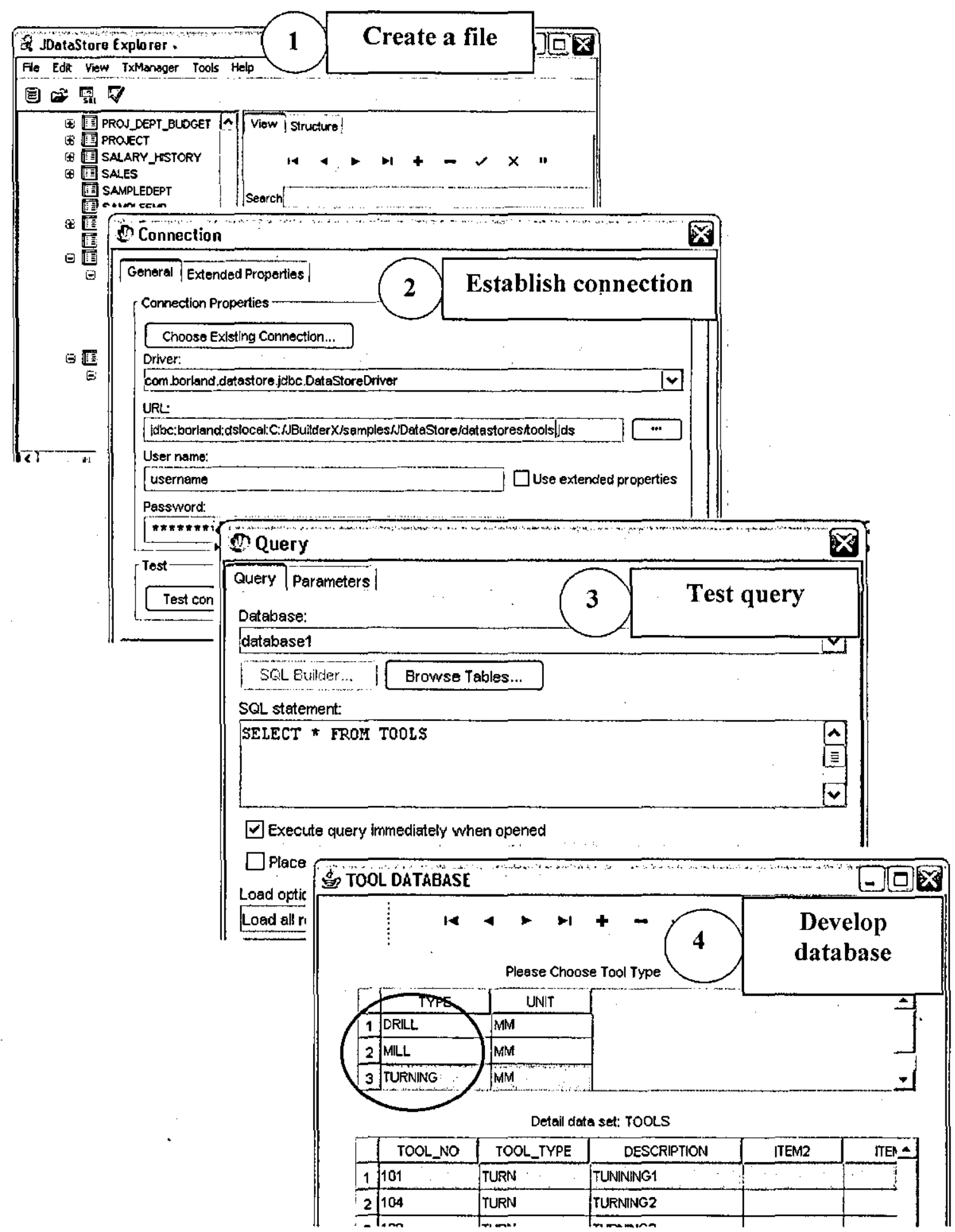

Figure 6.18 Tool database system for SCSTO 


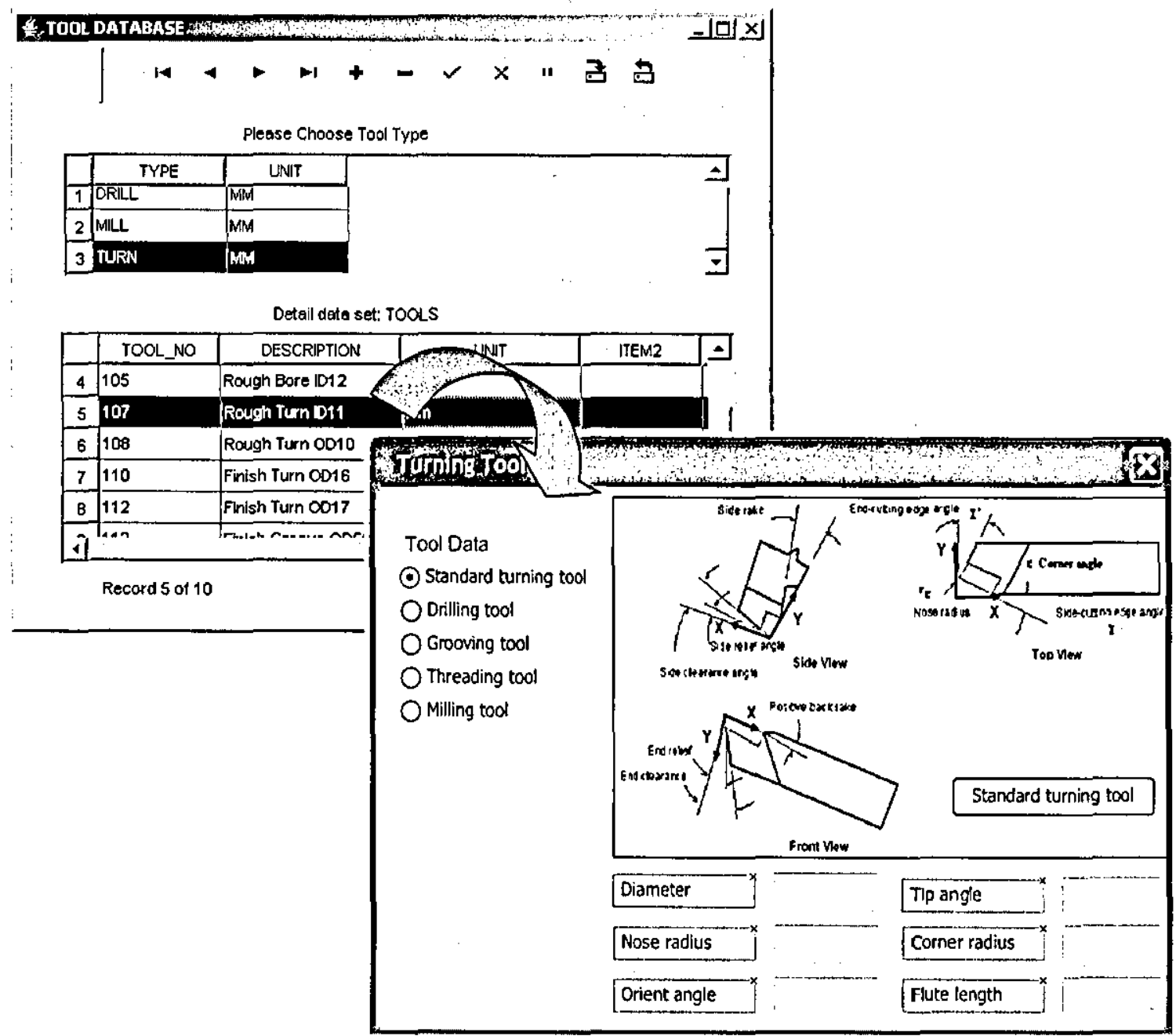

Figure 6.19 Turning tool dialog

\subsubsection{Turning Machining Strategies}

ISO 146499 Part 12 (ISO, 2005a) defines six turning machining strategies which are subtypes of the machining_strategy defined in ISO 14649-10 (ISO, 2004a). They are used to create the turning tool path. The turning machining strategies have been applied as shown in figure 6.20 (ISO schema) and 6.21 (java programming). 
ENTITY turning_machining_strategy

ABSTRACT SUPERTYPE OF (ONEOF (unidirectional_turning,

bidirectional_turning, thread_strategy, contour_turning,

grooving_strategy,explicit_turning_strategy));

overcut_length : OPTIONAL length_measure;

allow_multiple_passes : OPTIONAL BOOLEAN;

cutting_depth : LIST[0:?] OF length_measure;

variable_feedrate : OPTIONAL positive_ratio_measure;

END_ENTITY;

Figure 6.20 The turning_machining_strategy (ISO 14649-12 2004)

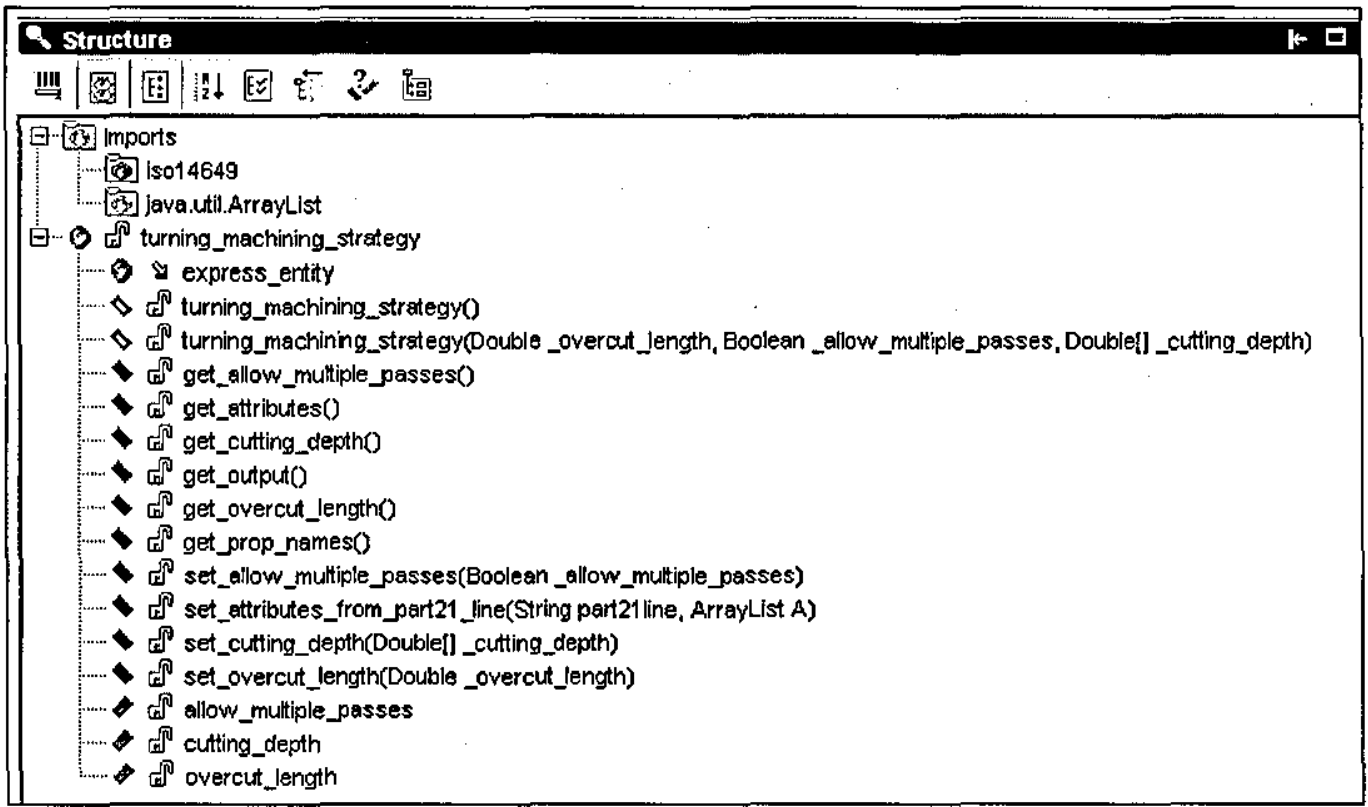

Figure 6.21 The Turning machining strategy

The users have the option to choose the machining strategy from the machining strategy menu as shown in figure 6.22. 


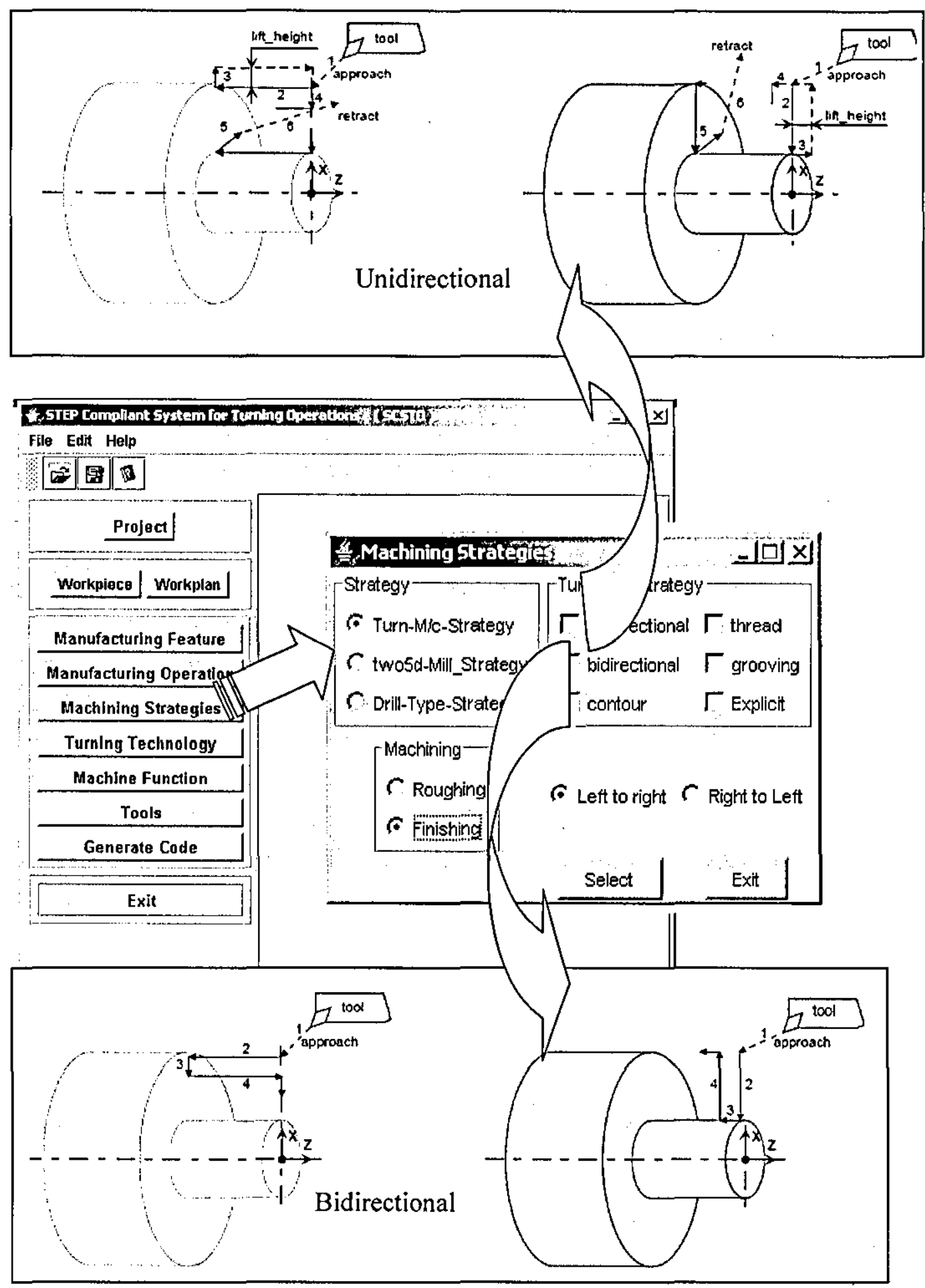

Figure 6.22 Turning machining strategies 
For example if the user is looking for roughing machining, it is better to choose bidirectional turning rather than unidirectional, to make sure that the insert tool is not damaged. Otherwise for a better result in finishing it is better to use unidirectional turning, due to feed rate being less than roughing.

\subsection{SCSTO Dialog}

Tool selection is one of the most important functions in a process planning system as it effects the selection of machining parameters, production rate, cost of product and the resulting accuracy (Usher and Fernandes, 1999). Therefore a tool database has been developed in SCSTO as shown in figure 6.23. The overall system dialog and the output file are presented in figures 6.24 . 


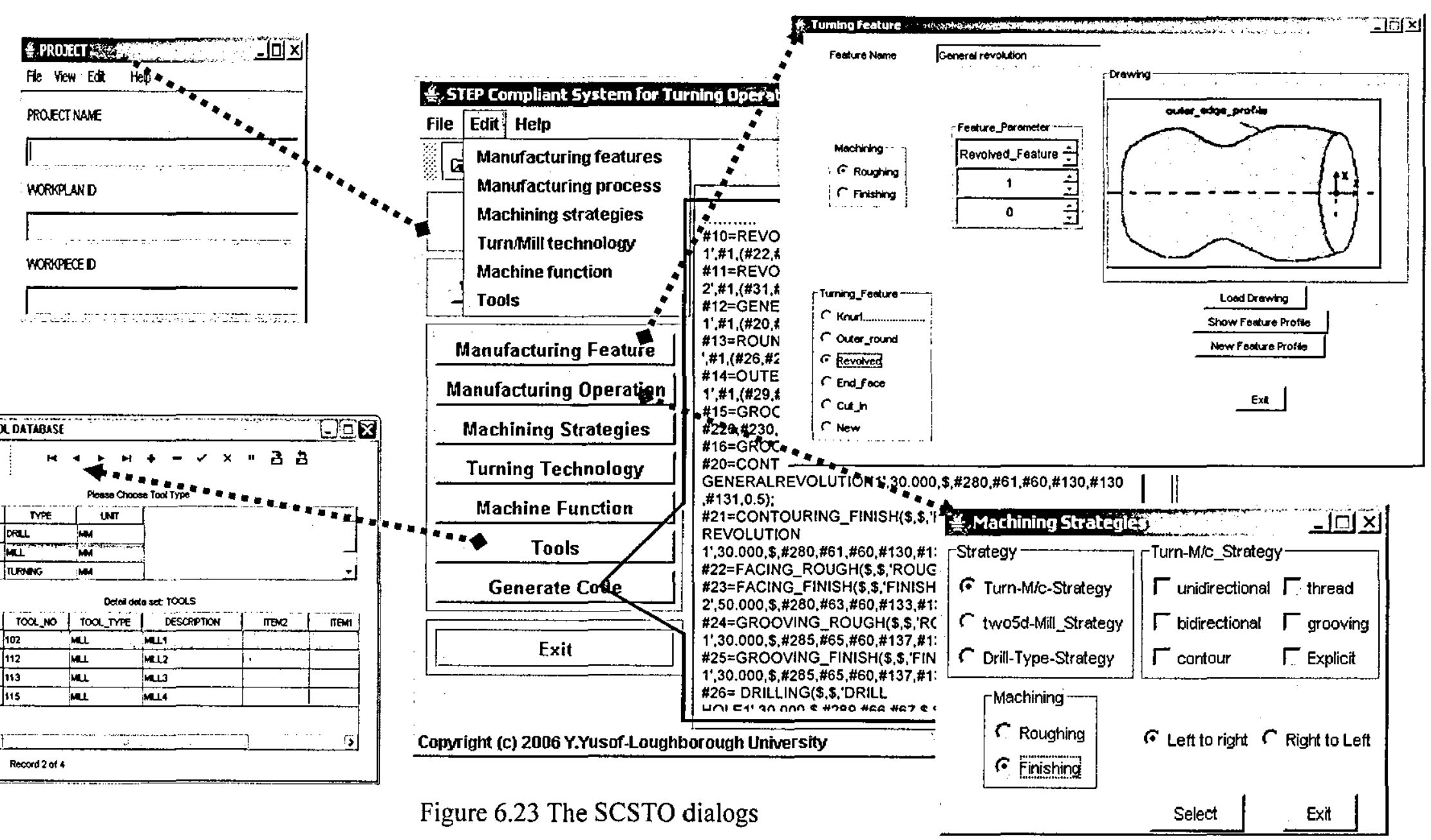




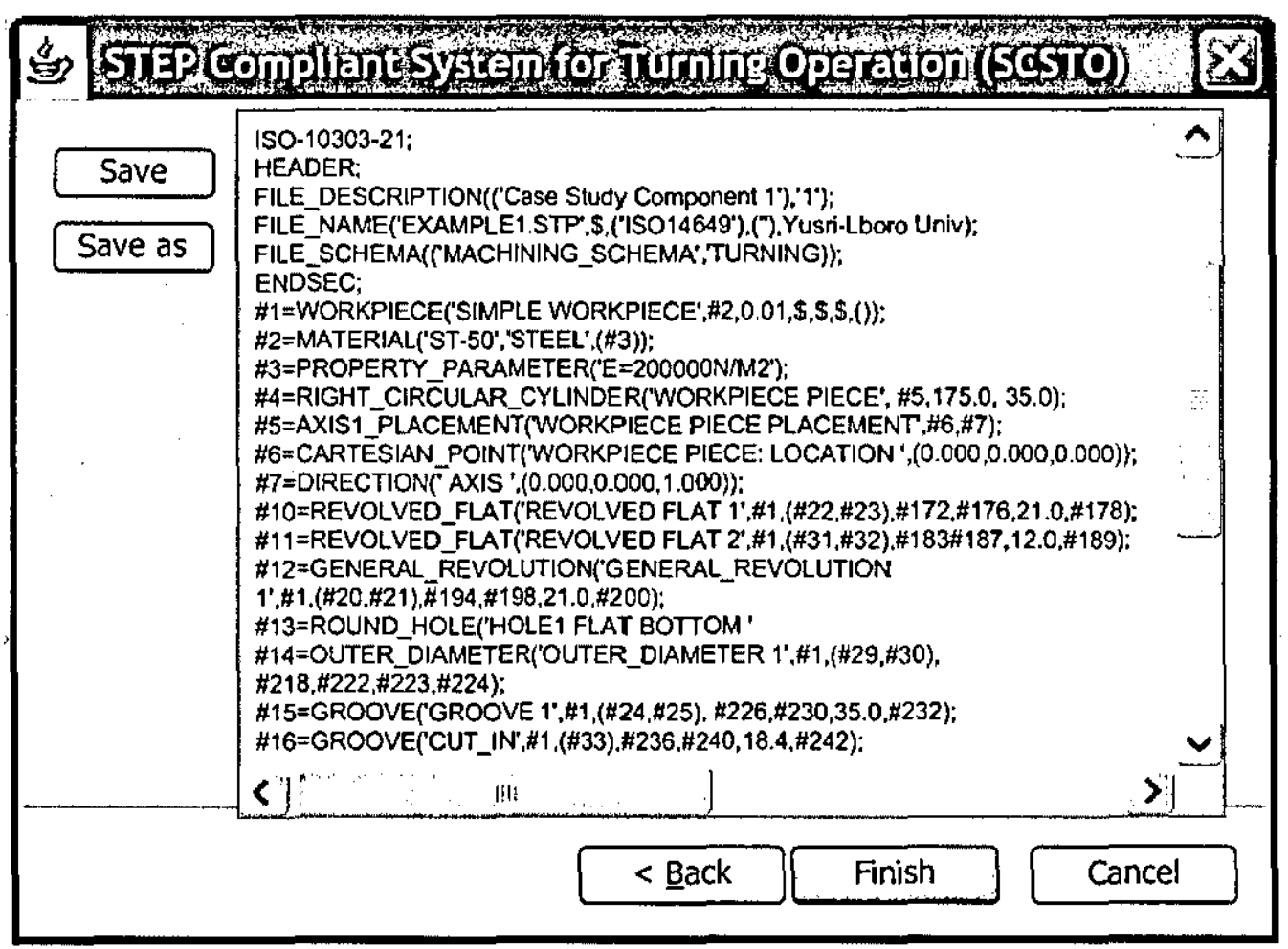

Figure 6.24 The output file

\subsection{Edit Menu}

As shown in figure 6.1, the system has an "Editor" function to make modifications either at the turning or operation stage. By using the file menu, edit pull down, the user can choose what he/she wants to edit. For example if users choose turning feature, the turning feature window will be displayed as shown in figure 6.25. This looping process will continue until the user is satisfied with the output. 


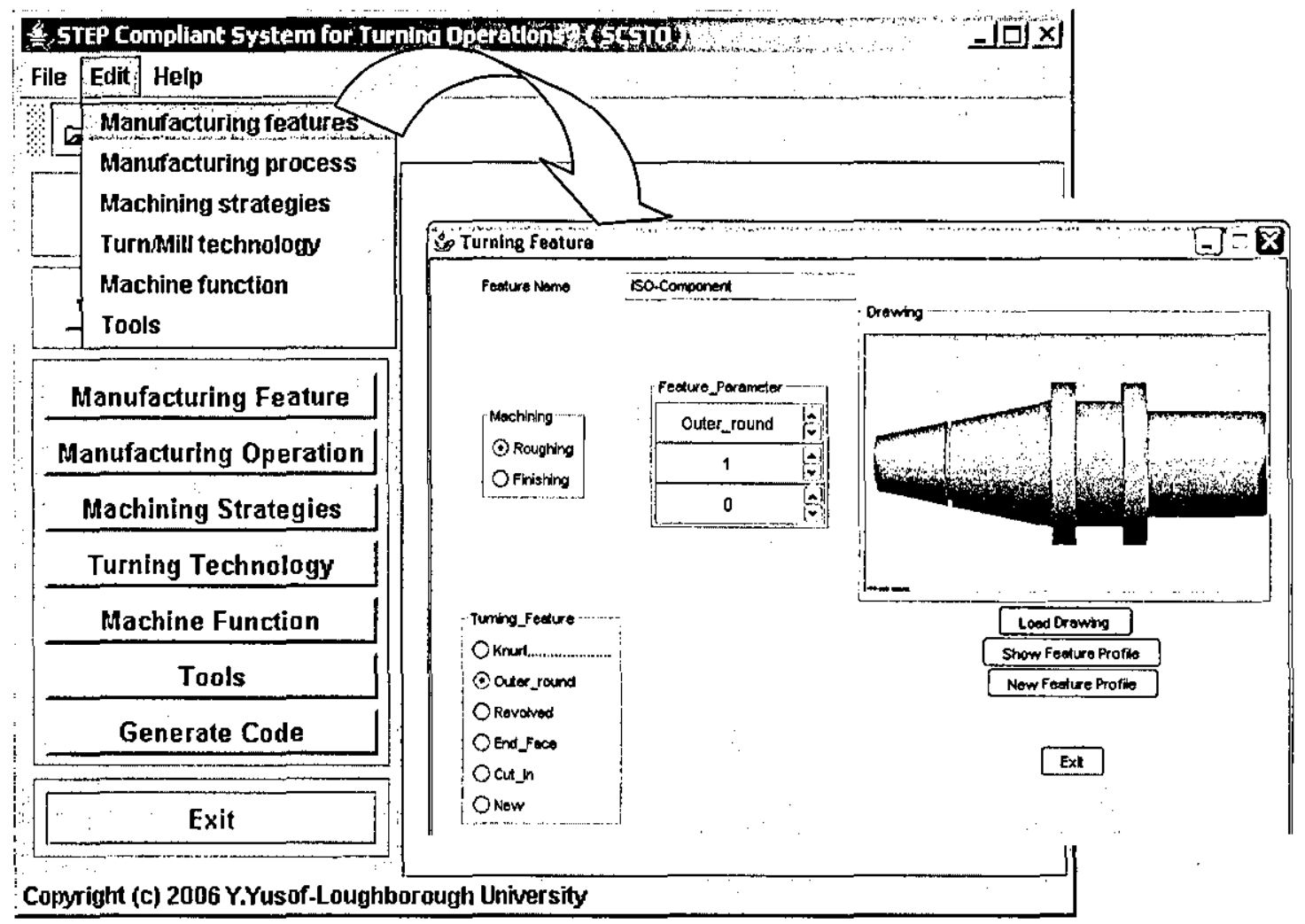

Figure 6.25 Edit menu in SCSTO environment

\subsection{Summary}

This chapter has presented the STEP compliant system for turning operations namely SCSTO. It began with the requirements and assumptions and was followed by an overview of the system. Then tools developed for the system and SCSTO architecture were discussed. All this development was needed to cover objective number four, which was to develop the prototype system of SCSTO. Through the implementation of SCSTO one of the objectives of this research is realised in the creation of a computational environment for a STEP-NC compliant system for turning operations. It also demonstrates the validity and effectiveness of the data model. The next chapter discuss the implementation of two case studies. 


\section{Chapter 7 - Case Study Implementation}

\subsection{Introduction}

In this chapter, case studies have been conducted to explore the capabilities of the proposed system as stated in the final objective in Chapter 1. The following sections explain the case study implementation, with only first component being explained in detail. The second component is only briefly described in this chapter. For component 1 , the main agenda is to confirm that the prototype system using the feature-based approach. The second component is used to prove the capability of the prototype system to read the AP203 file format generated from commercial CAD software. Details are provided in sections 7.3 and 7.4.

\subsection{Case Study Components}

This chapter discusses two case study components, which are designed by using the developed features and have been chosen from established ISO documents and an established World Class Company. The first case study describes an example in ISO 14649-12 (ISO, 2005) and the second is an industrial component from Mazak (Mazak, 2002). The first component is used to demonstrate the feature extraction technique and the second component is used to demonstrate the direct transfer from a CAD file format (STEP 203) to the proposed system and the generation of the ISO 14649 part program. The main reason for choosing the first component was that it provided an increasing complexity in terms of the number of 
features that it contains. This first component requires a simple configuration that represents the first level of $\mathrm{CNC}$ turning where only $\mathrm{x}, \mathrm{z}$ and $\mathrm{z}$ axes are available (e.g. Okuma and Homa model HL35M). Components requiring a second set-up are manually rotated and positioned and re-held in the chuck unless the machine has a counter spindle in which case it can be machined in a single set-up.

The components were selected for three reasons; i) component number 1 , is taken from ISO 14649 Part 12 (ISO, 2005). ii) they have increasing overall complexity, according to a combination number of $\mathrm{CNC}$ machine axes, and iii) they contain specific aspects such as, a number of features and a number of set-ups. Details are shown in figure 7.1.

\begin{tabular}{|r|c|c|}
\hline No & Description & Graphic \\
\hline 1 & $\begin{array}{c}\text { Component 1 } \\
\text { Example from ISO } \\
\text { 14649-12 }\end{array}$ & \\
2 & $\begin{array}{c}\text { Component 2 } \\
\text { (Turn/Mill c-axis } \\
\text { machined component with } \\
\text { double chucking }\end{array}$ & \\
\hline
\end{tabular}

Figure 7.1: Case Study Components

\subsection{Case Study Component 1}

The first component provides an understanding about the wide variety of inner and outer features and their orientations that can be used in the design of 
rotational components. The overall size for the component is $170 \mathrm{~mm} \times \varnothing 70 \mathrm{~mm}$. These dimensions are given considering the feature geometric attributes and absolute placement considering machining and tool aspects. Each feature of the component is labelled and machining operations are turning, grooving and drilling. This component has double sided asymmetrical general attributes and seven machining_features; two circular_faces, two outer_diameters, one general_revolution, one groove, and one cut_in as shown in figure 7.2. The final shape is machined in two setups; setup one is for right side features and second setup is for left side features.

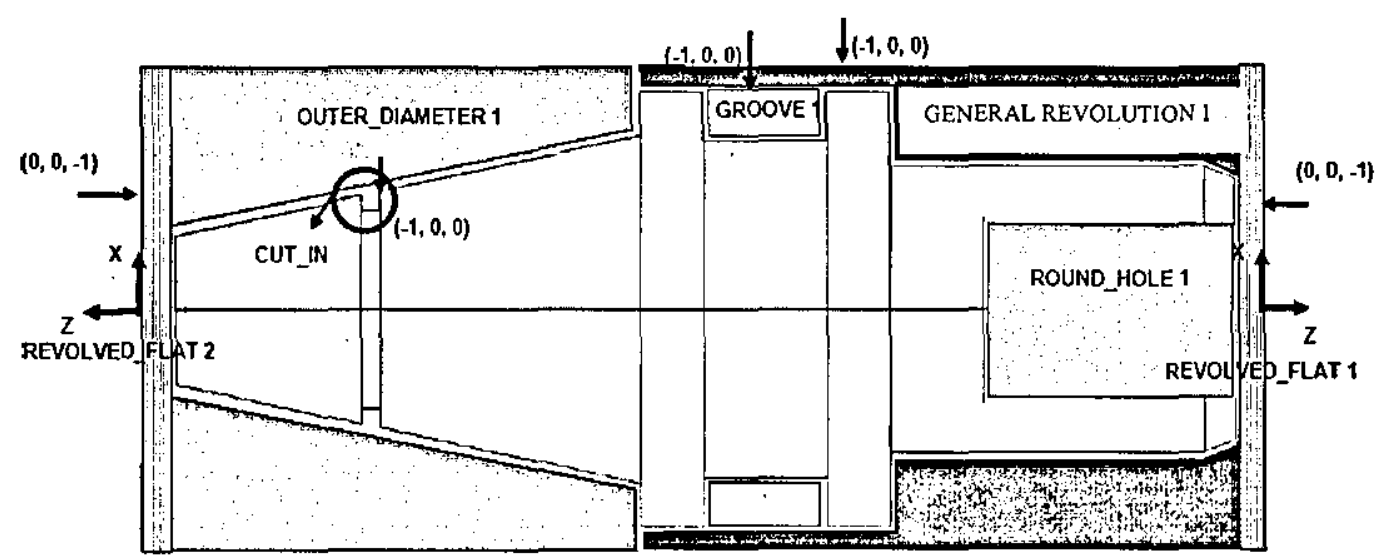

Figure 7.2 Machining features in case study component 1 (ISO, 2005a)

Figure 7.3 presents a 3D solid view of component number 1 to provide a better understanding of the $3 \mathrm{D}$ geometry. 


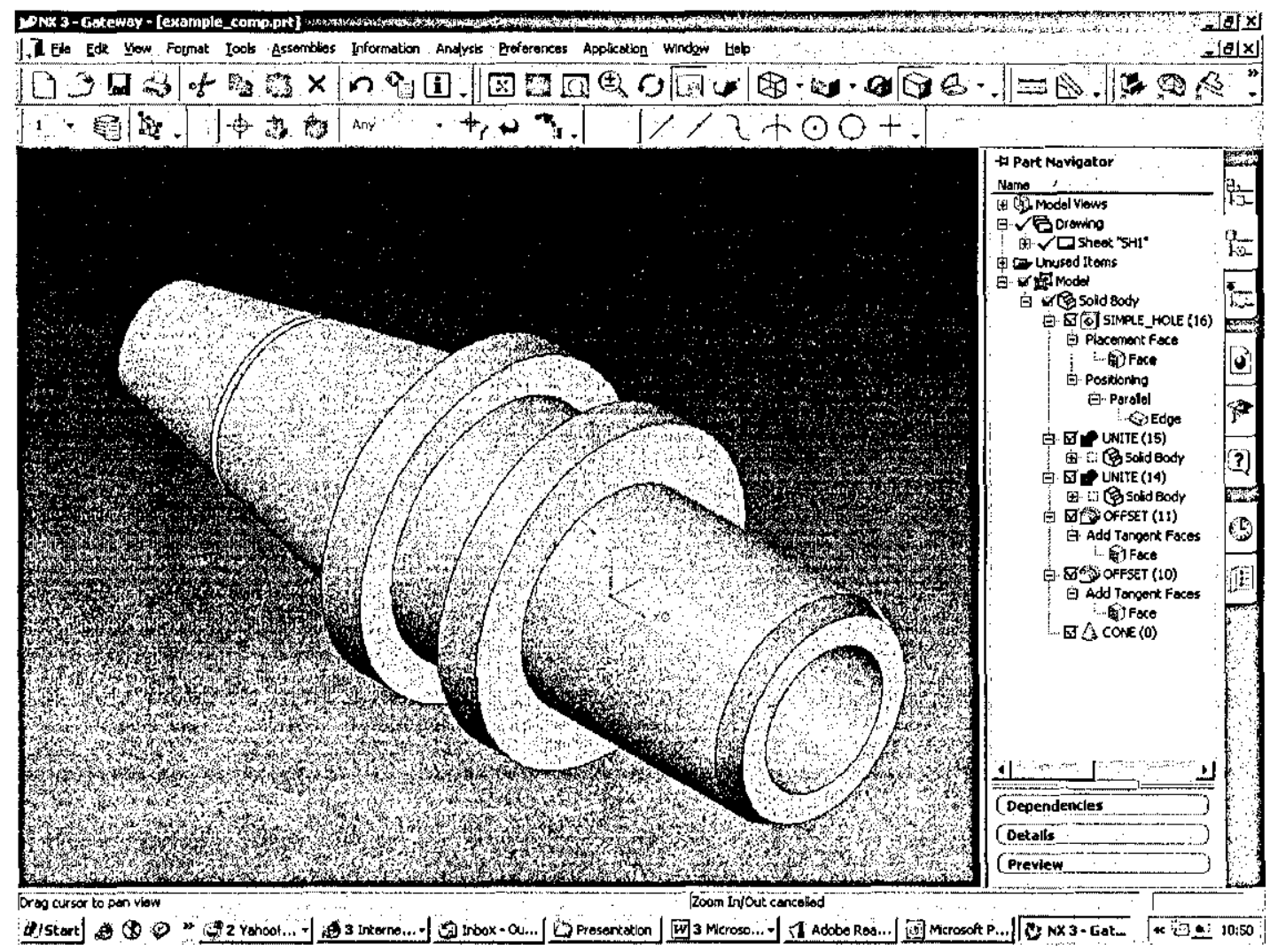

Figure 7.3 3D solid Unigraphic view of case study component no 1

\subsubsection{Manufacturing Process}

The requirements on the machining process in this case are that the toolpaths shall be as continuous as possible and with as few direction changes as possible to prevent marks on the surfaces. The operations plan for this component consists of some $37 \mathrm{NC}$-sequences. The machining starts with the set-up I as shown in figure 7.4. Firstly a roughing sequence removes material as fast as possible. The goal for this sequence is to remove material inside the inner hole. After this the component is taken out of the machine and turned around into set-up II, so that the machining can be carried on from the other side. First a roughing sequence which is followed by one or more turning sequences. After the roughing, a finishing process is carried out. 


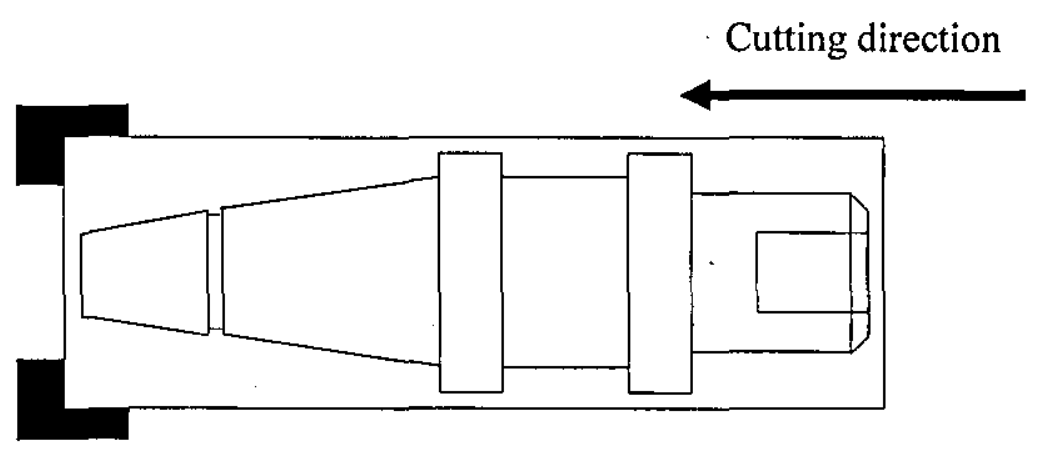

First Set-up (I)

\section{Cutting direction}

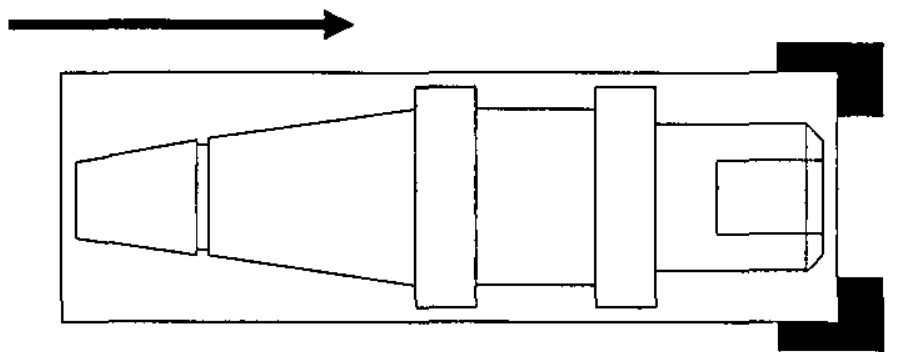

Second set-up (II)

Figure 7.4 Top (upper) and section (lower) views of case study component no 1

The starting point of the machining operations required for producing an asymmetrical component as case study component 1 , is to consider the operations possible on the $\mathrm{CNC}$ turning machine as shown in figure 7.5. In this case study, the machine configurations were limited to;

i. standard turning machine with 2 axes ( $\mathrm{x}$ and $\mathrm{z}$ ), marked as " $\mathrm{A}$ "

ii. 3 axis CNC machine ( $x, z$ and $c$ ), marked as " $B$ "

iii. 4 axis CNC machine (one spindle and two turrets), marked as "C"

iv. 6 axis $\mathrm{CNC}$ machine (two spindles and two turrets), marked as "D"

Table 7.1 shows the possibilities of producing this component depending on the type of CNC machine and with consideration of the number of set-ups. Machines A, B and C need a second set-up to be done manually. Machines A, B 
and $\mathrm{C}$ need to stop the spindle and setup again at the machine spindle for machining side II. For more efficiency, machine $\mathrm{D}$ is the best solution due to using only 1 setup. It transfers automatically to the counter spindle and this capability saves a lot of time.

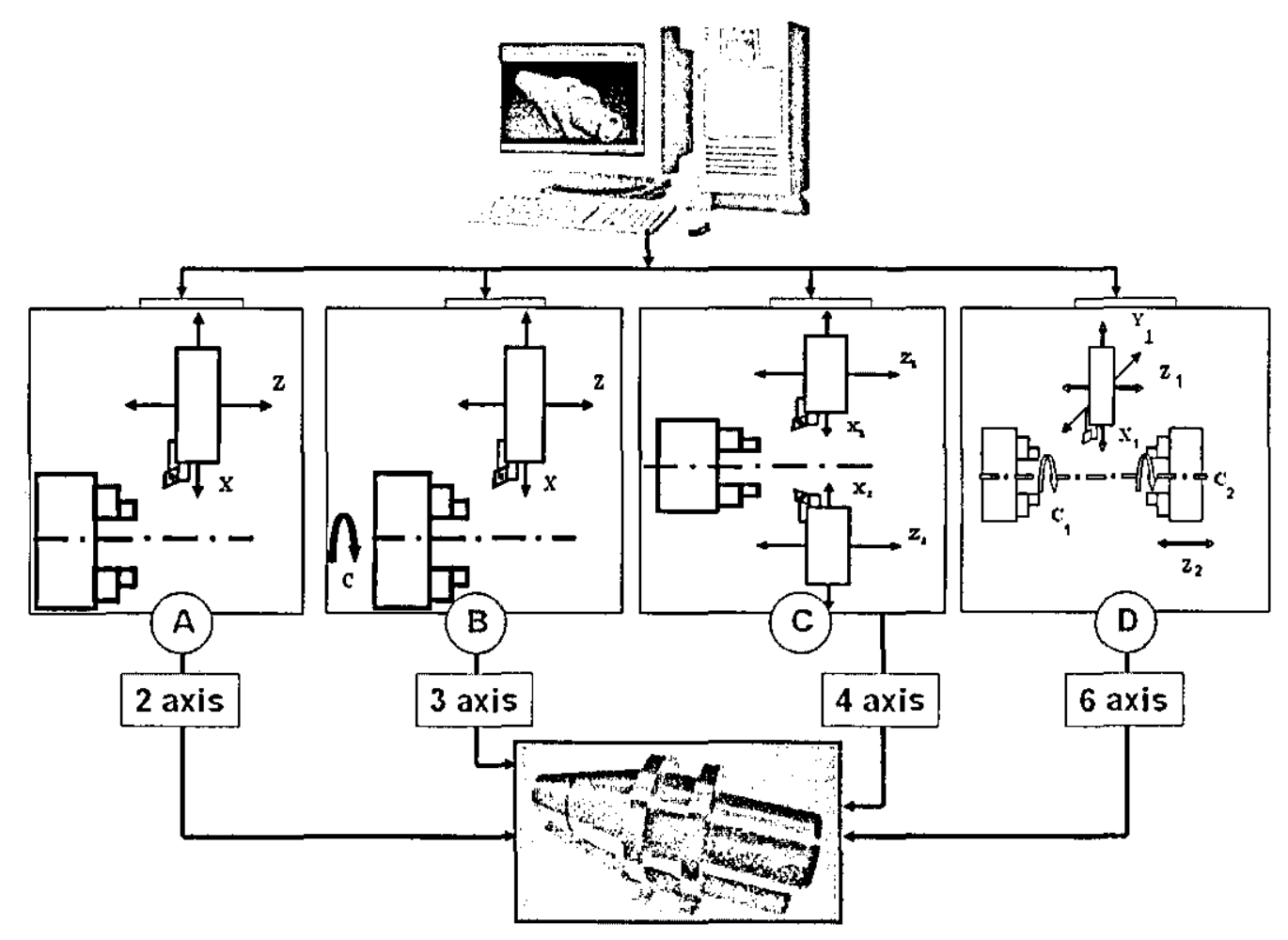

Figure 7.5 the various $\mathrm{CNC}$ machine for case study component no 1

Table 7.1 Turning machine configurations

\begin{tabular}{|l|c|c|l|}
\hline No & Machine & $\begin{array}{r}\text { Number } \\
\text { of Set- } \\
\text { ups }\end{array}$ & \multicolumn{1}{|c|}{ Description (refer to figure 7.5) } \\
\hline 1 & A & 2 & $\begin{array}{l}\text { Better machining side I first, rather then side II } \\
\text { due to better gripping on the stock material at the } \\
\text { second set-up. Only uses x and } \mathrm{z} \text { axes. }\end{array}$ \\
\hline 2 & B & 2 & Same as machine A. \\
\hline
\end{tabular}


Table 7.1 Turning machine configurations (continued)

\begin{tabular}{|l|c|c|l|}
\hline No & Machine & $\begin{array}{c}\text { Number } \\
\text { of Set- } \\
\text { ups }\end{array}$ & \multicolumn{1}{|c|}{ Description (refer to figure 7.5) } \\
\hline 3 & C & 2 & $\begin{array}{l}\text { Same as machine A, but tool changes can be } \\
\text { minimised because this type of machine has } \\
\text { double turrets, providing full 4-axis } \\
\text { representation of the upper and lower turret. } \\
\text { Synchronisation of machining can be use for } \\
\text { maximum efficiency. }\end{array}$ \\
\hline $\mathbf{4}$ & D & $\mathbf{1}$ & $\begin{array}{l}\text { This type of CNC machine needs only 1 set-up, } \\
\text { machining side I first and then transferring to the } \\
\text { counter spindle to complete the component by } \\
\text { machining side II. }\end{array}$ \\
\hline
\end{tabular}

\subsubsection{Operations by SCSTO}

When SCSTO starts, the starting window is displayed as shown in figure 7.6. The project, workplan and workpiece ID information is filled as shown in figure 6.13 for the header element. SCSTO continues by defining a project in the header statement as mentioned in chapter 6 . This project statement includes information about the main workplan and refers to the PROJECT entity that has its identifier defined as a STRING and it includes the WORKPIECE classes. In the case of component 1 , the project name is "turning example", work plan has three items, the main work plan, the workplan for set-up 1 and the workplan for set-up 2 as shown in figure 7.7. Finally the workpiece is defined as simple workpiece and the material chosen for this case study is aluminium. 


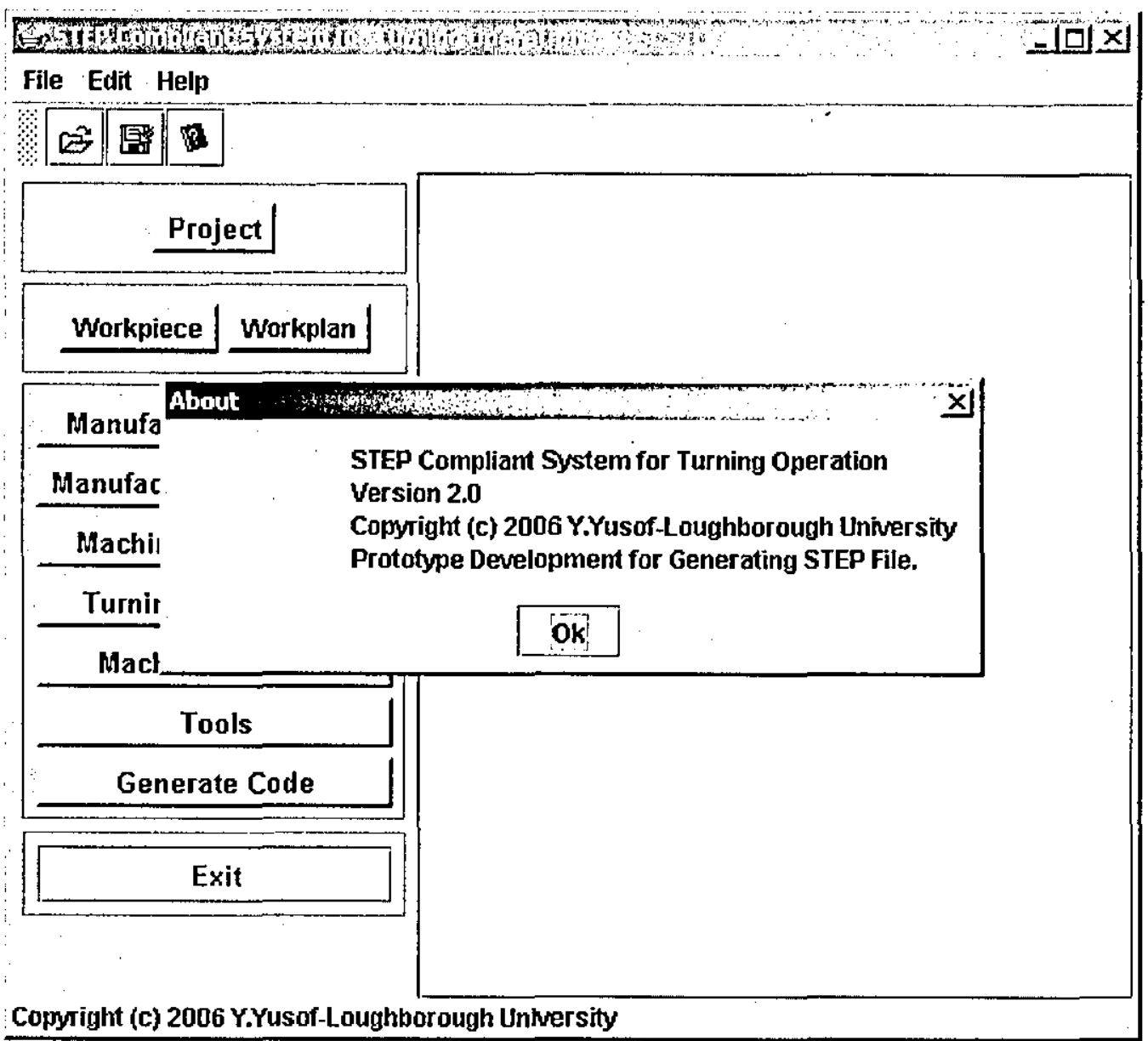

Figure 7.6 SCSTO main window dialog

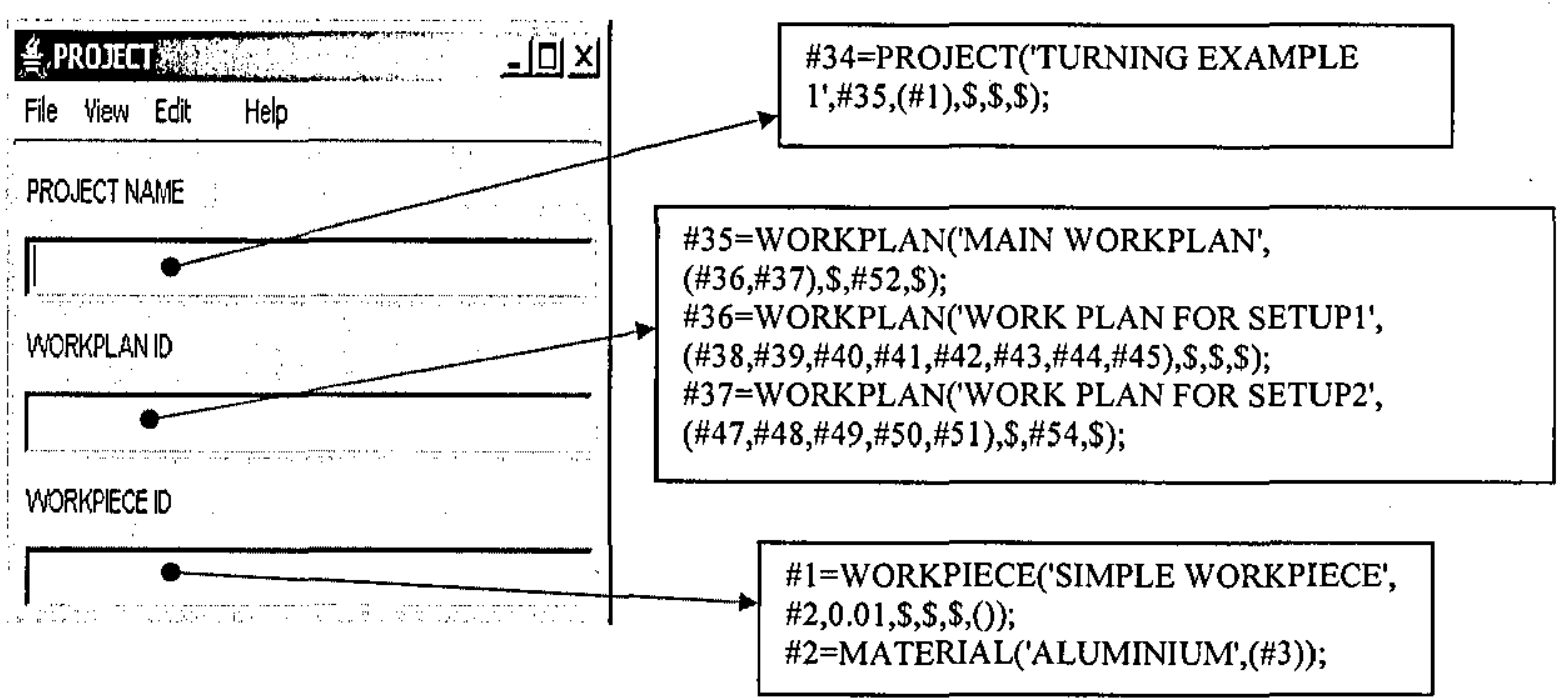

Figure 7.7 SCSTO Project dialog. 
This demonstration for case study component 1 , starts with the new project under menu file as mentioned in 6.3 . for new feature creation by the users. In the cylindrical base dialog the overall size, location and number of setups are defined. This shape is considered as the starting raw material for modelling the component. Then the user creates the features and attaches them to the base part. The set-up selection refers to attaching the workpiece into the spindle dependent on the machine configuration as shown in figure 7.5.

As clarified earlier the purpose of this component is to confirm the capabilities of the prototype system using the new feature approach. The operations involved in this case are;

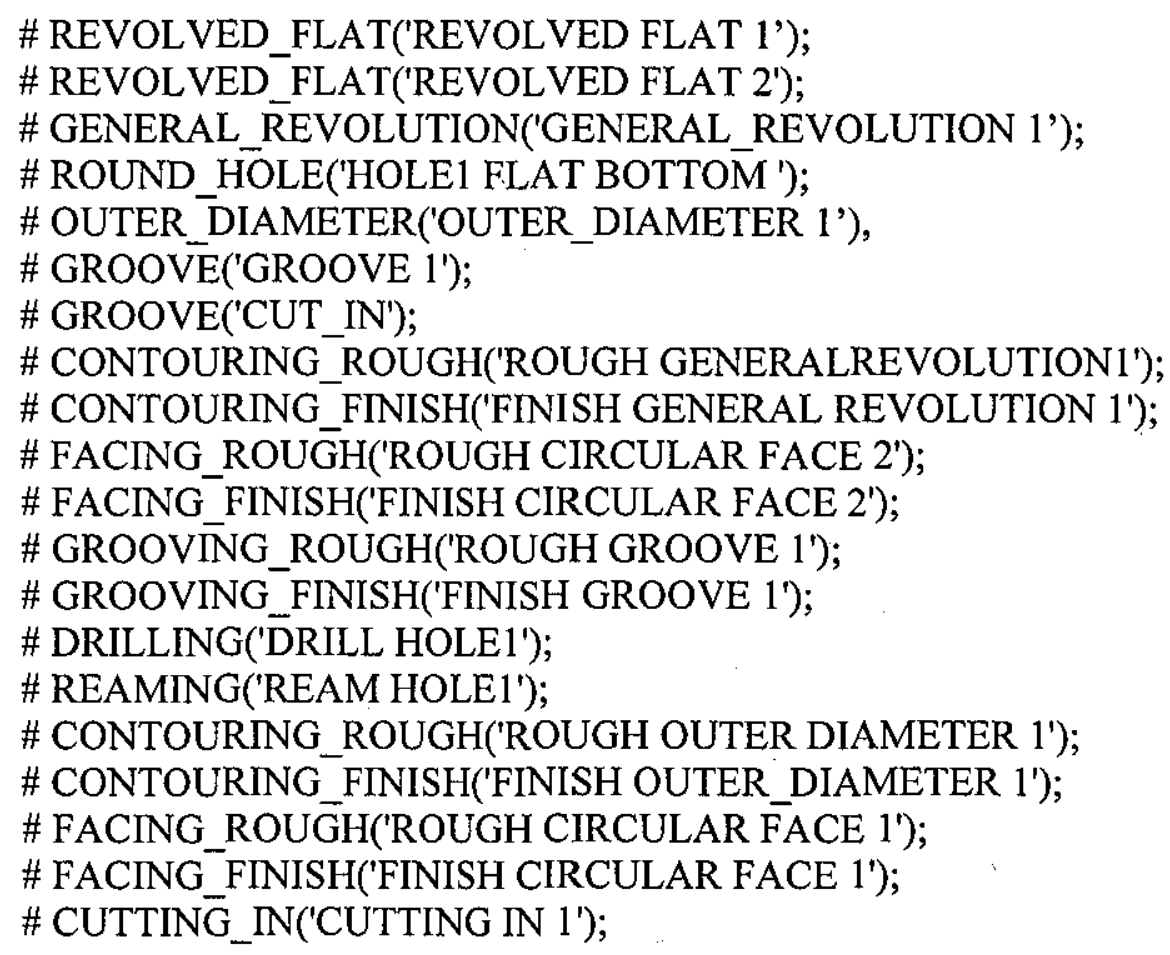

All the features created by the user need to be oriented to the base part which in this prototype is limited to cylindrical shapes as shown in figure 7.8. Combo box control is used to determine the number of set-ups and can be chosen to be either one or two. 


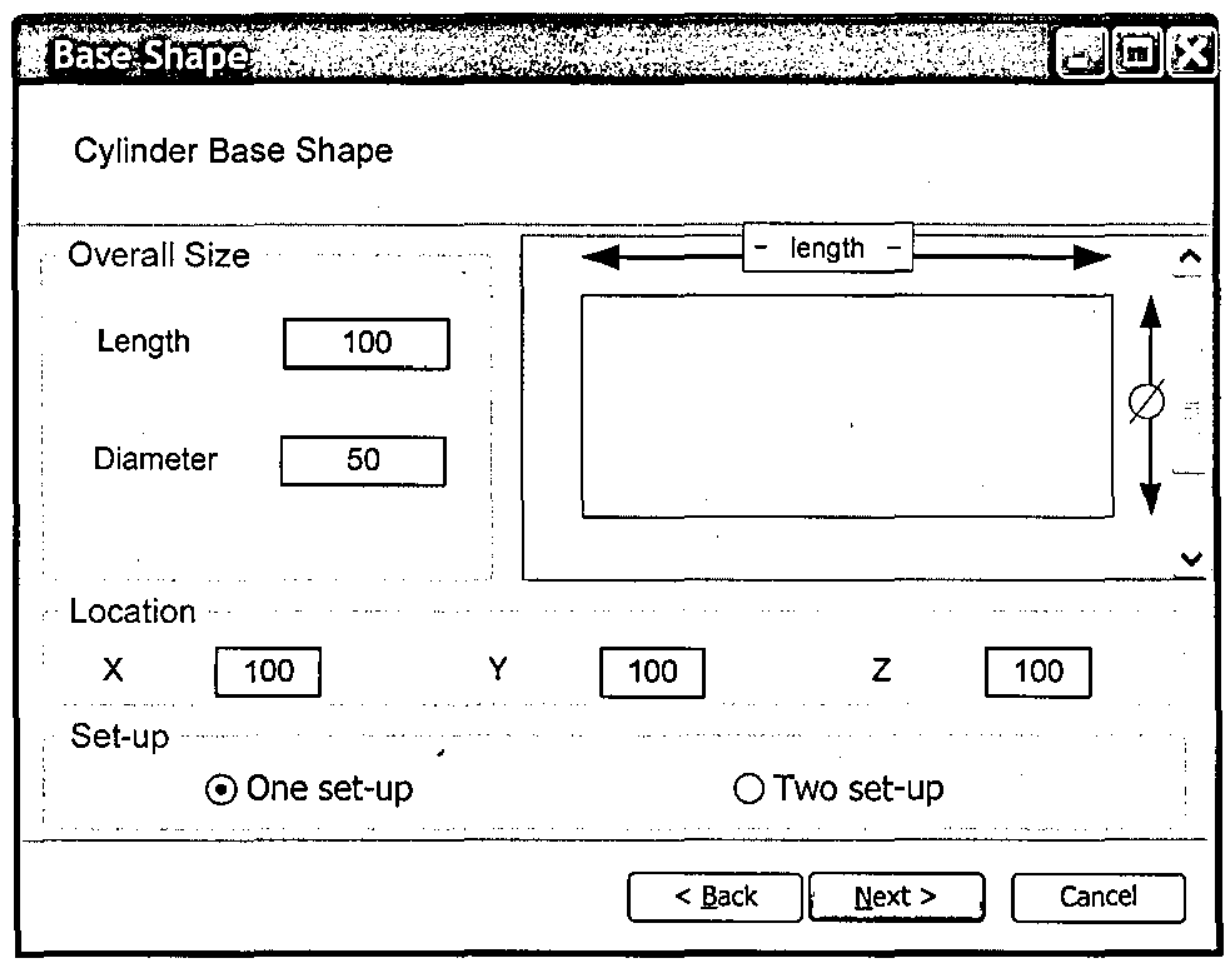

Figure 7.8 Cylinder base dialog

After creating the base component, the features attachment process can be started. As shown in figure 7.9 turning features can be selected from radio button controls. The user can start with any feature dependent on user defined manufacturing considerations by clicking the New button. In turning operations, the type of machining such as roughing or finishing is also important for tool selection. This can be defined by clicking radio button control for roughing or finishing. 


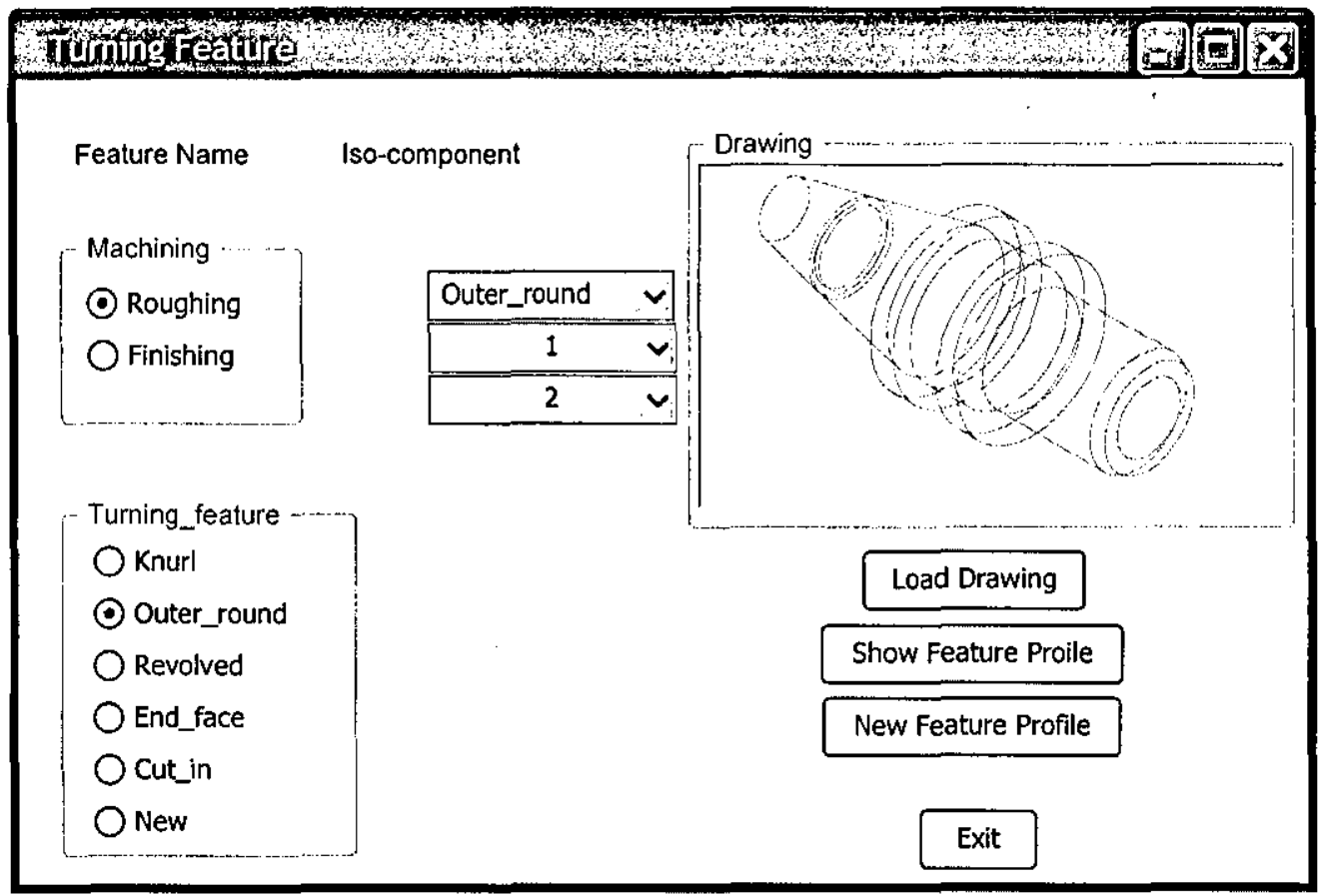

Figure 7.9 Turning feature dialog

The turning strategy for this case study component as shown in figure 7.10 can be selected from;

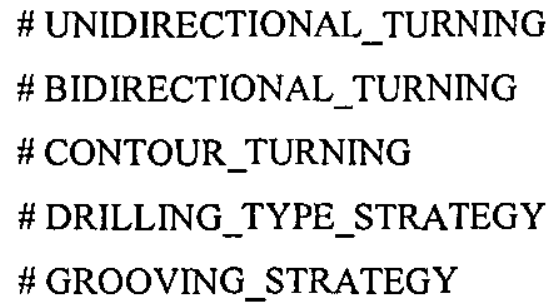




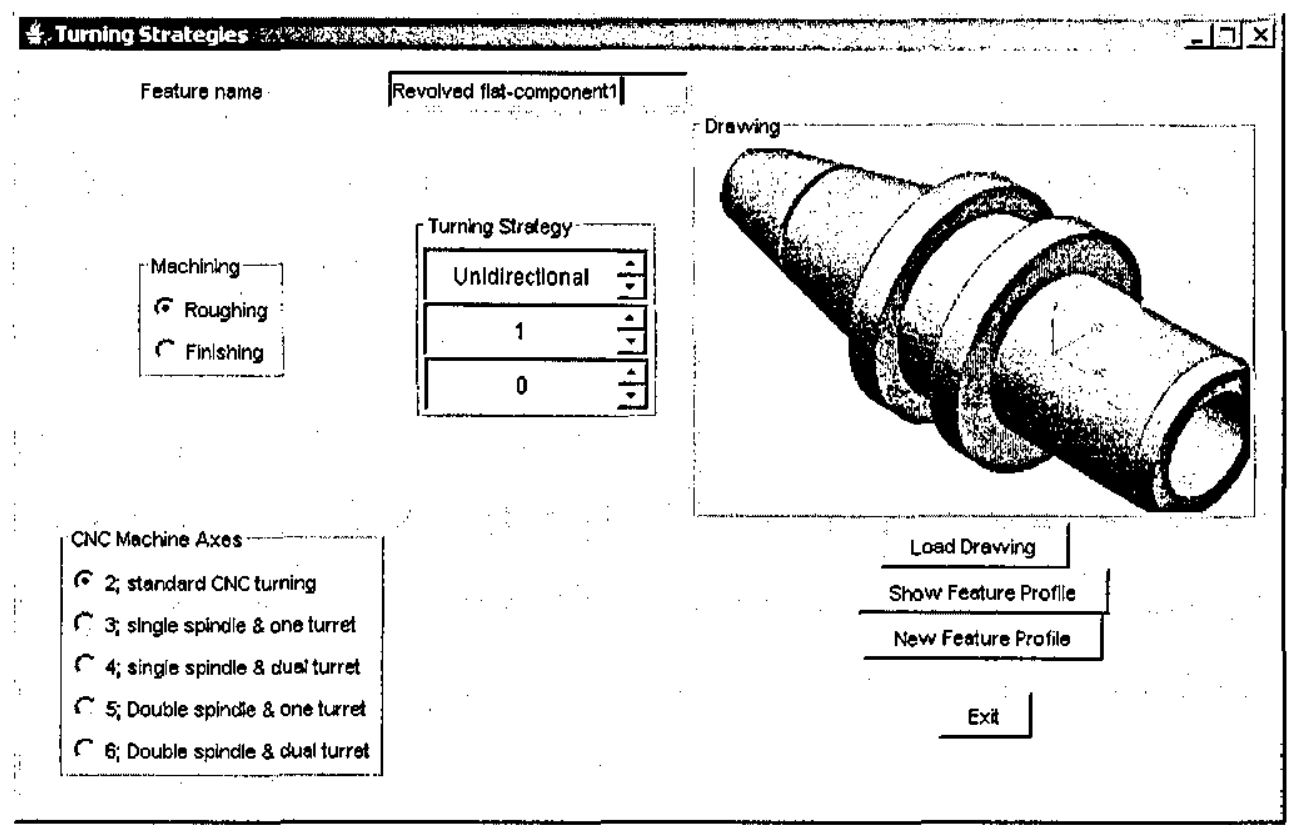

Figure 7.10 Turning strategies dialog

The turning technology in this case refers to feed rate, spindle speed and feed per revolution as shown in Figure 7.11. This function button cannot be modified by the user as it is a default based on the machine capability. Similarly the machine function control for coolant is a default value.

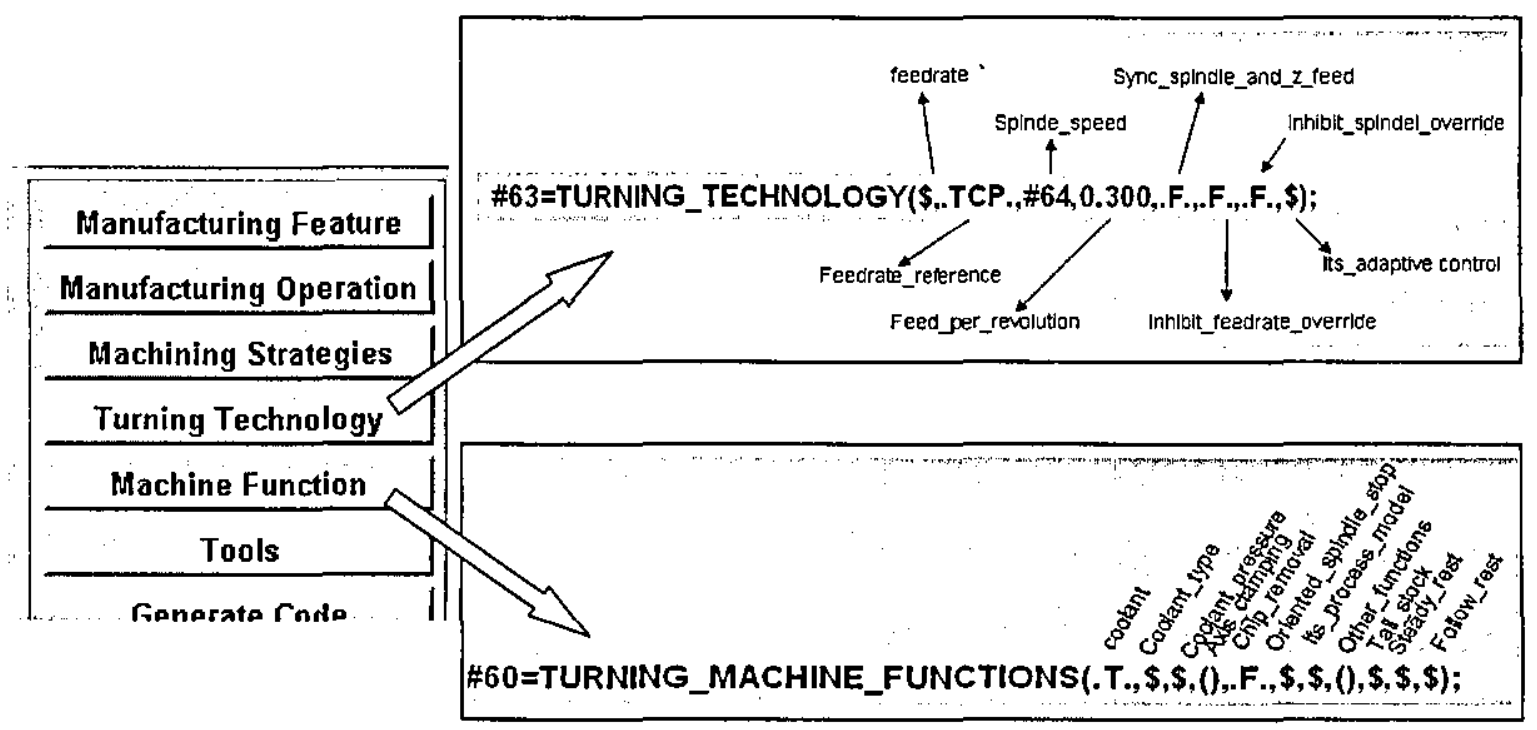

Figure 7.11 Turning technology and machine function dialog 
The cutting tool is selected only when a specific machine has been selected and uses a tool database based on ISO 14649 Part 121 (ISO, 2005b) as shown in figure 7.12. Each cutting tool can be edited by the user but depends on the machine capability. Figure 7.13 shows details of the cutting tool specifications.

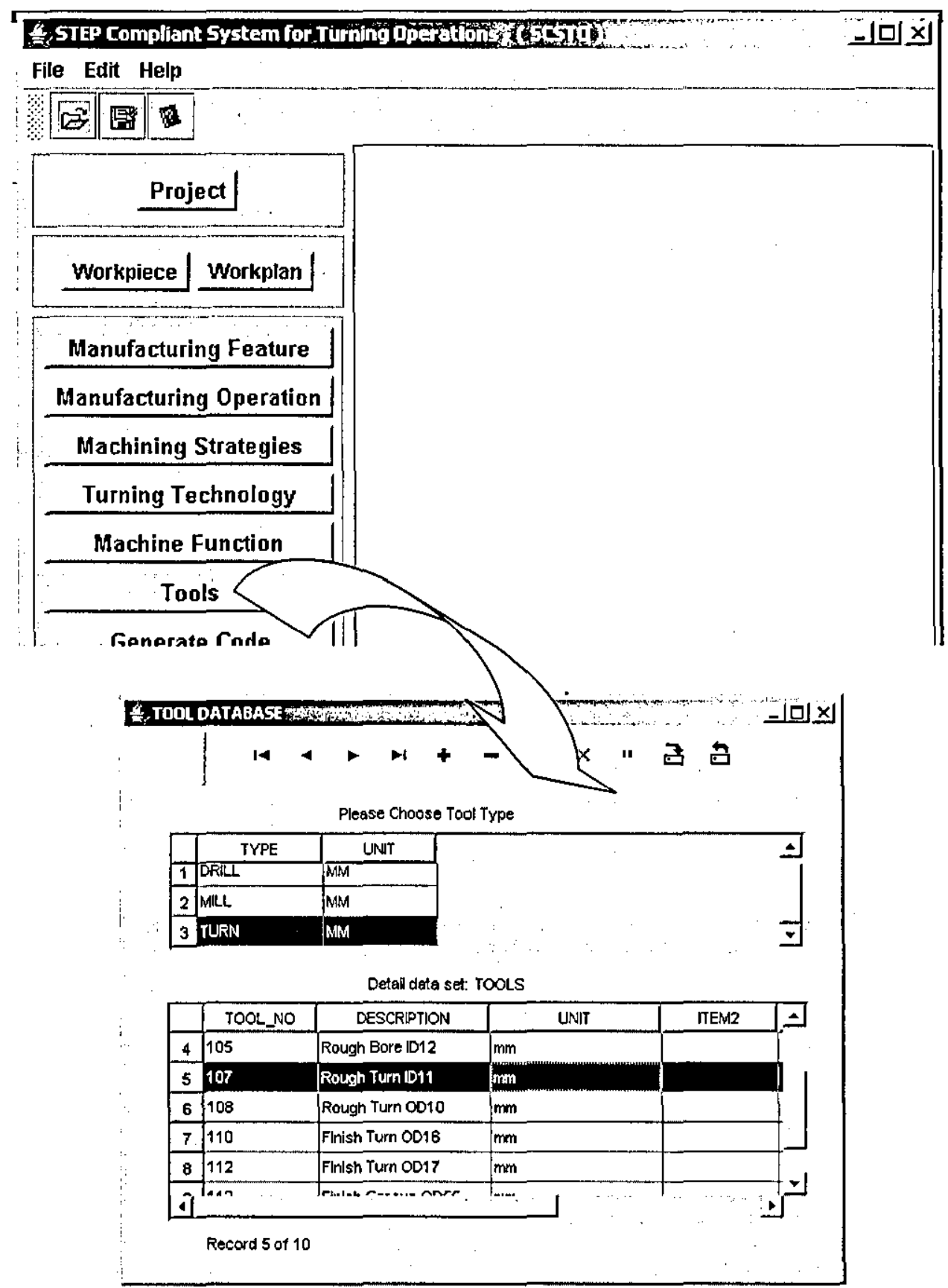

Figure 7.12.Cutting tool database 


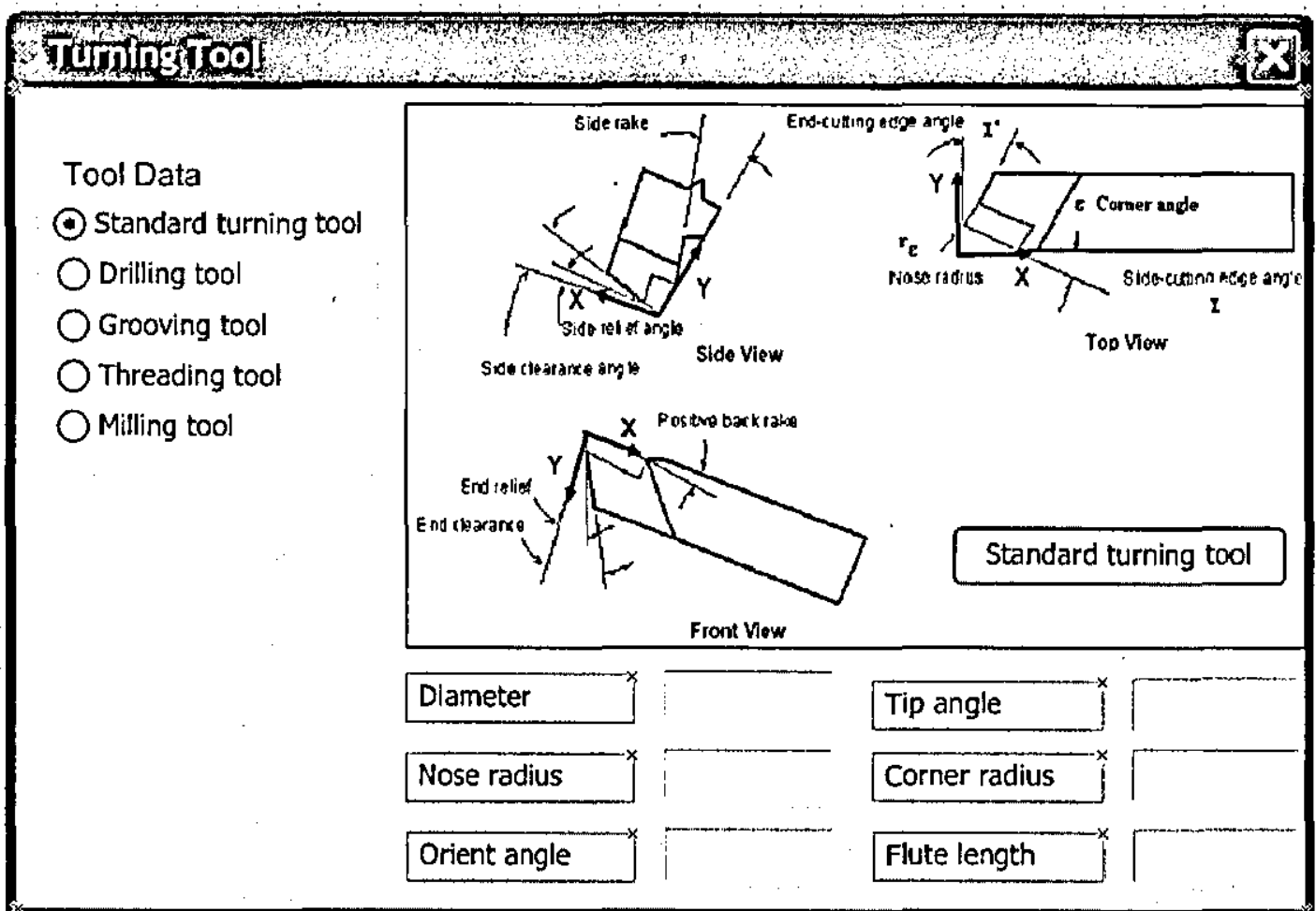

Figure 7.13. Cutting tool data

The system generates a workingstep and a workplan, building a tree structure that has a feature linked with those already defined in turning operations. Turning workingsteps are a list of turning features which are machined in sequence using the turning operations. The workplan follows primarily the setups as defined in the early stages of the process plan. Figure 7.14 depicts the dialog that appears just after the workplan has been created. 


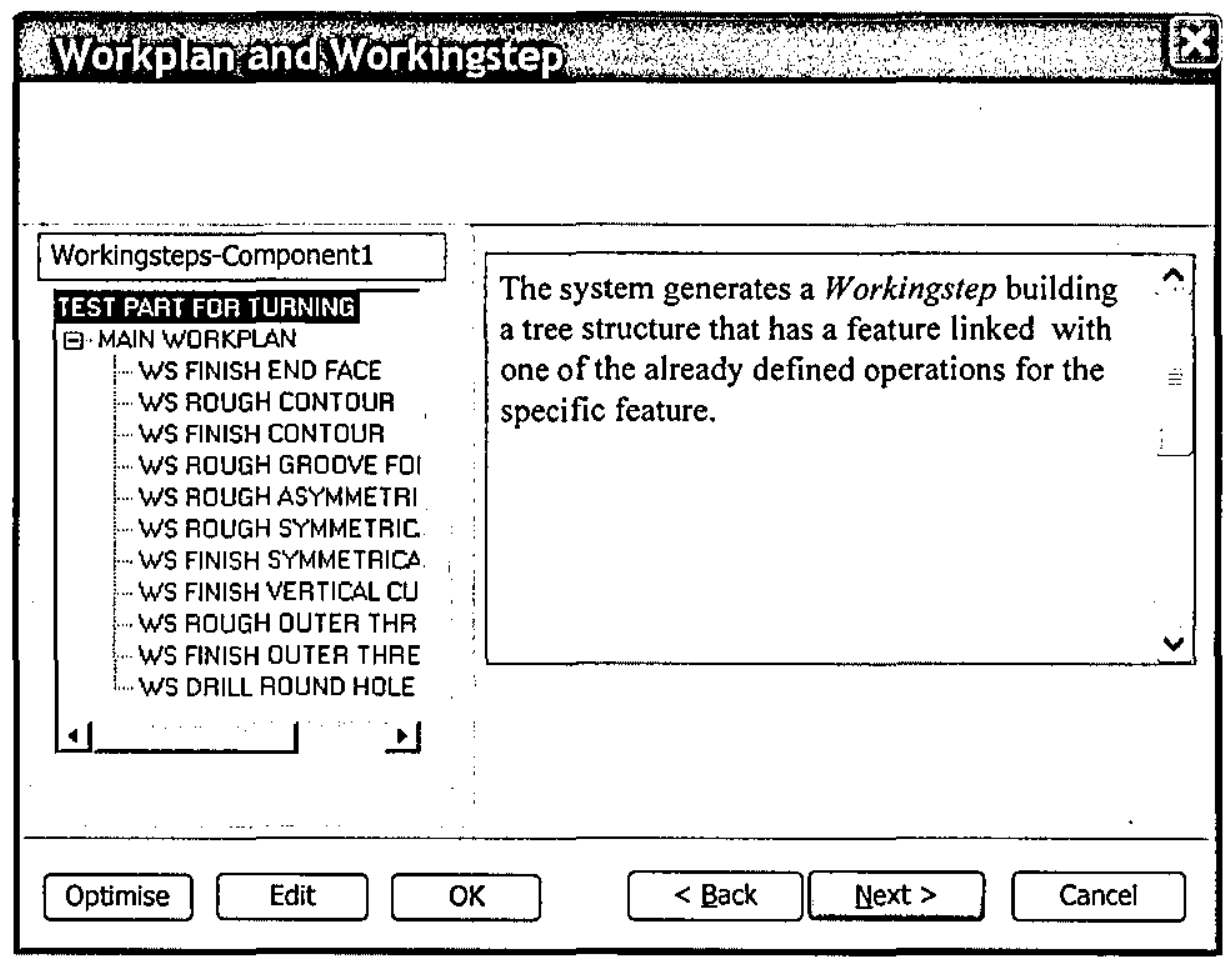

Figure 7.14 Workingsteps and workplan dialog

Finally, an ISO 14649 part program is generated by clicking the Generate Code button. The program is based on workpiece and machining_workingsteps in a physical file text format as shown in figure 7.15. This text file can be saved to a selected directory folder. As mentioned in sections 4.12 and 6.8 the part program can be edited by the user based on manufacturing features, strategies, tools, etc as shown in figure 7.16. When the user has finalised the part program, it can be sent to the machine controller. 


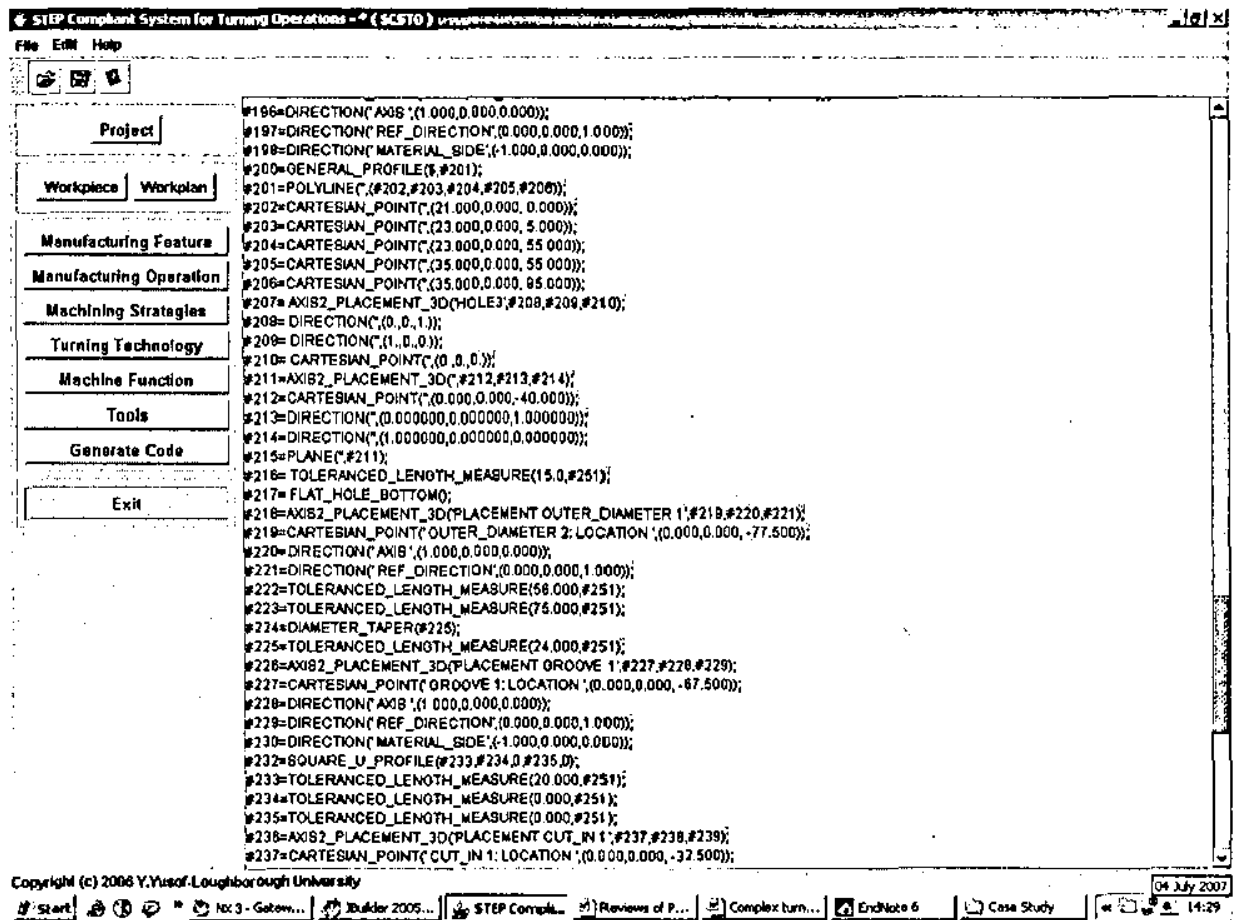

Figure 7.15 Part program generated by SCSTO

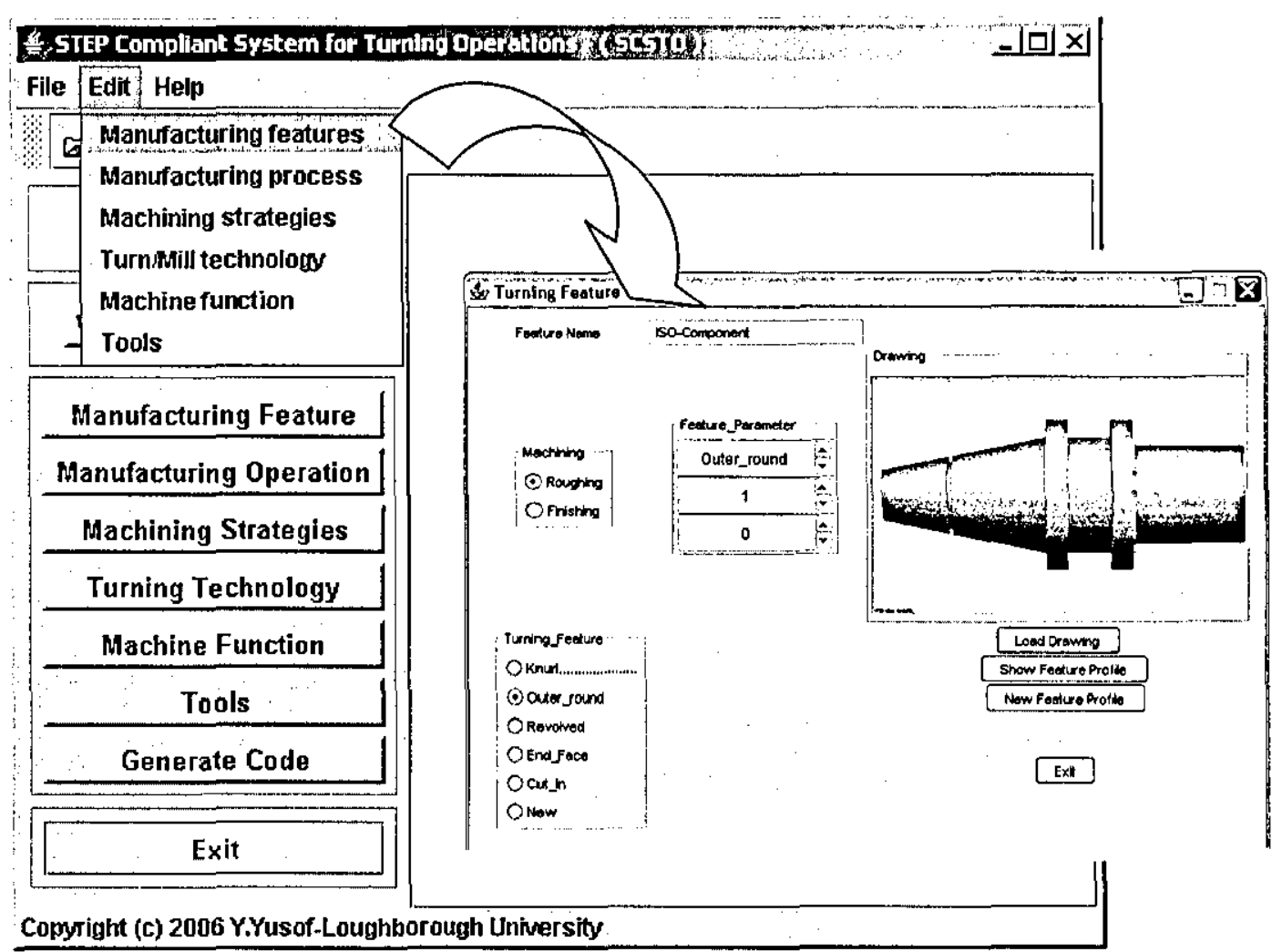

Figure 7.16 Possible editing for component 1 
After considering the available machine axes to produce the case study component 1 , SCSTO can generate the part program for machines A, B, C and D as shown in figure 7.17. Machines A, B and C must have two set-ups because they only have one spindle.

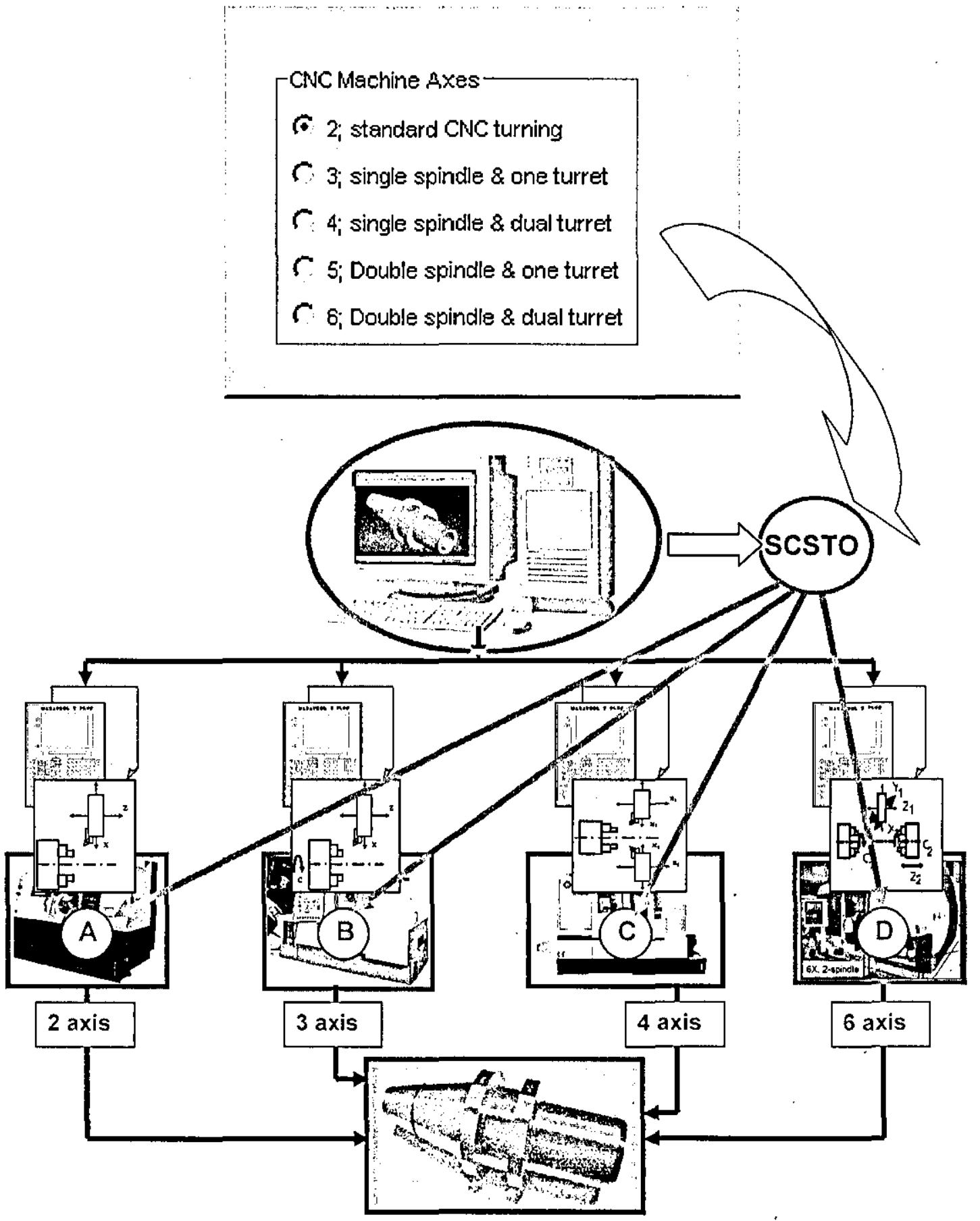

Figure 7.17 Possible machines for case study component 1 


\subsubsection{STEP Physical File Created by SCSTO}

The physical file codes of the roughing and the finishing processes for case study component 1 are summarised in table 7.2 and detailed in Appendix F.

Table 7.2 Physical File codes by SCSTO for component 1

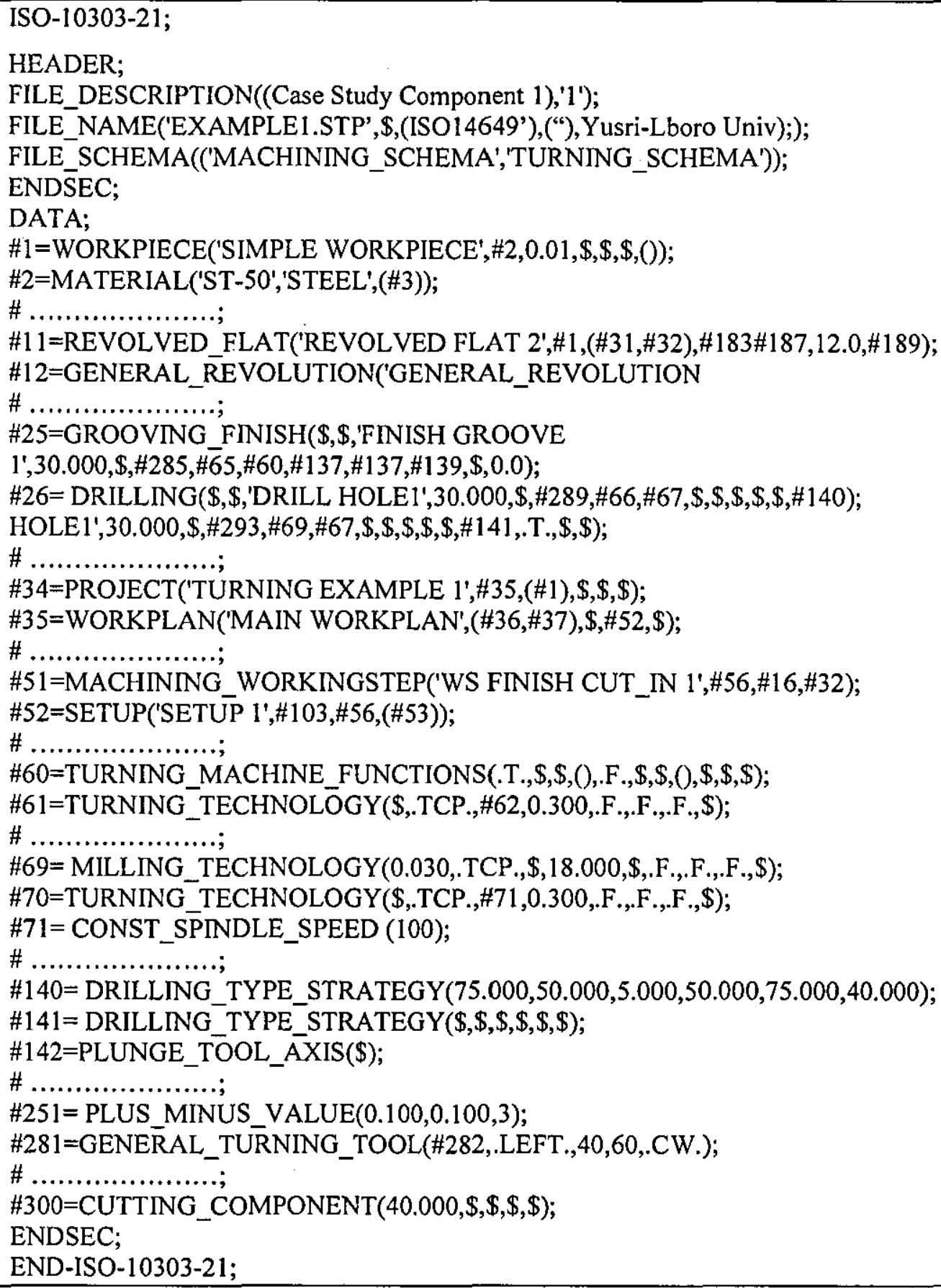




\subsubsection{NC Programming for Case Study Component 1}

The NC codes of the roughing and the finishing processes are summarised in table 7.3 and detailed in Appendix G

Table 7.3 G \& M Codes for component 1

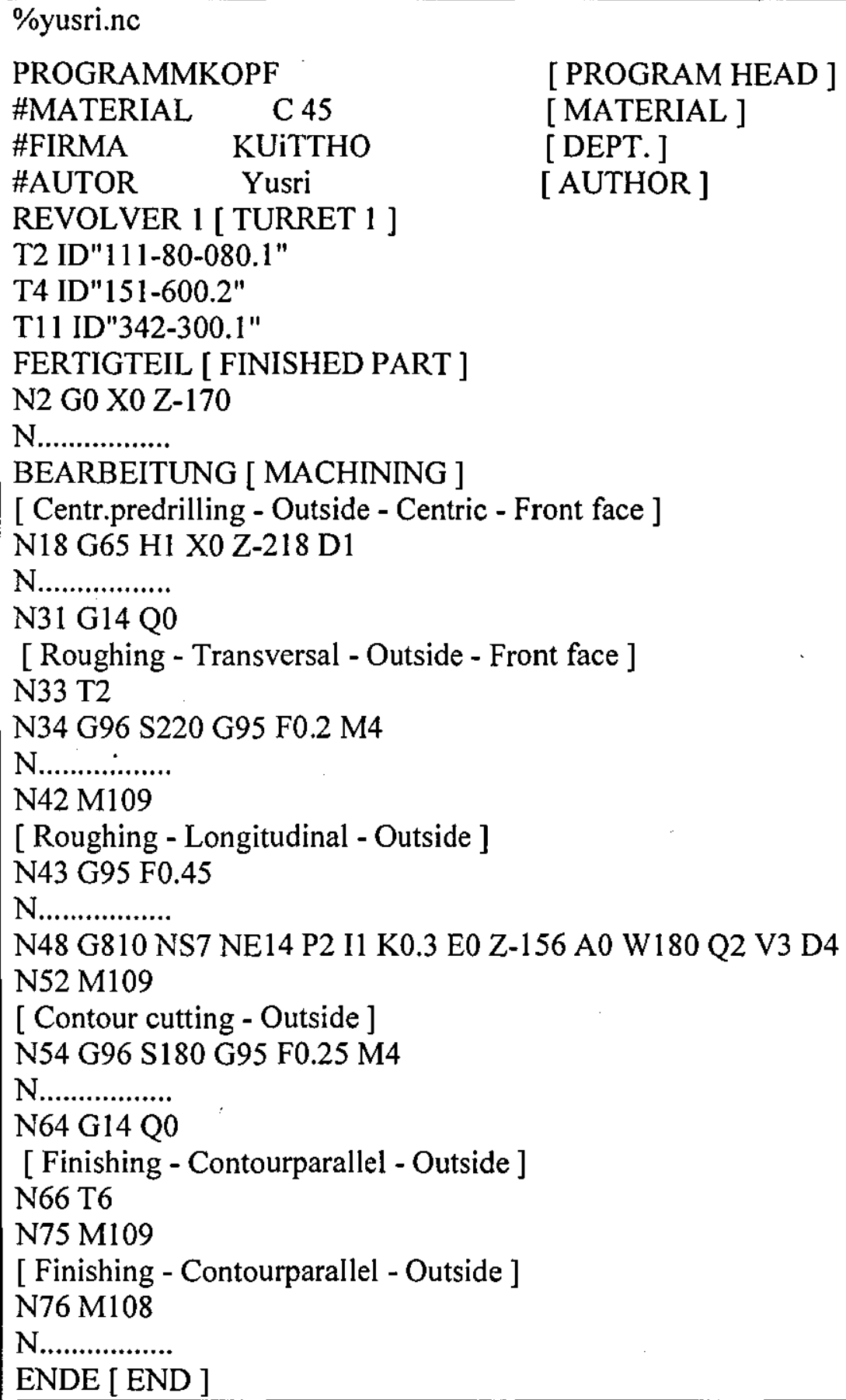




\subsection{Case Study Component 2}

Machining operations for case study component 2 are turning, grooving, threading, off centre drilling and milling on the side face. Its general attributes are double-sided and asymmetrical. The main contribution of this case study is to show the ability of SCSTO to read from a CAD file and recognise all the features after which it follows the same process as component 1 with definition of turning technology, turning strategies, defining tools, workplan and workinsteps. This description focuses on the imported file and the recognition of the features. The rest of the process is very similar to section 7.3 . Figures 7.18 and 7.19 present the 3D solid and isometric views of component 2 to provide a better understanding of the 3D geometry. Table 7.4 shows the detailed features including machining operations.

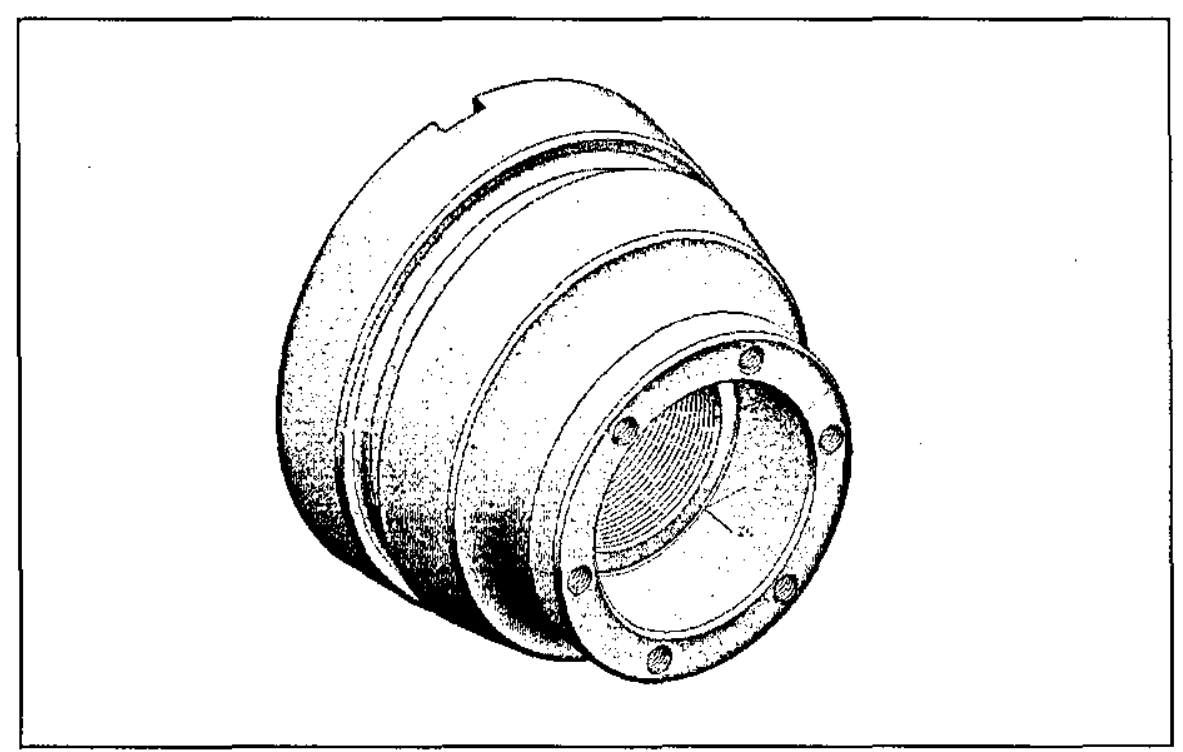

Figure $7.183 \mathrm{D}$ drawing for component no 2 


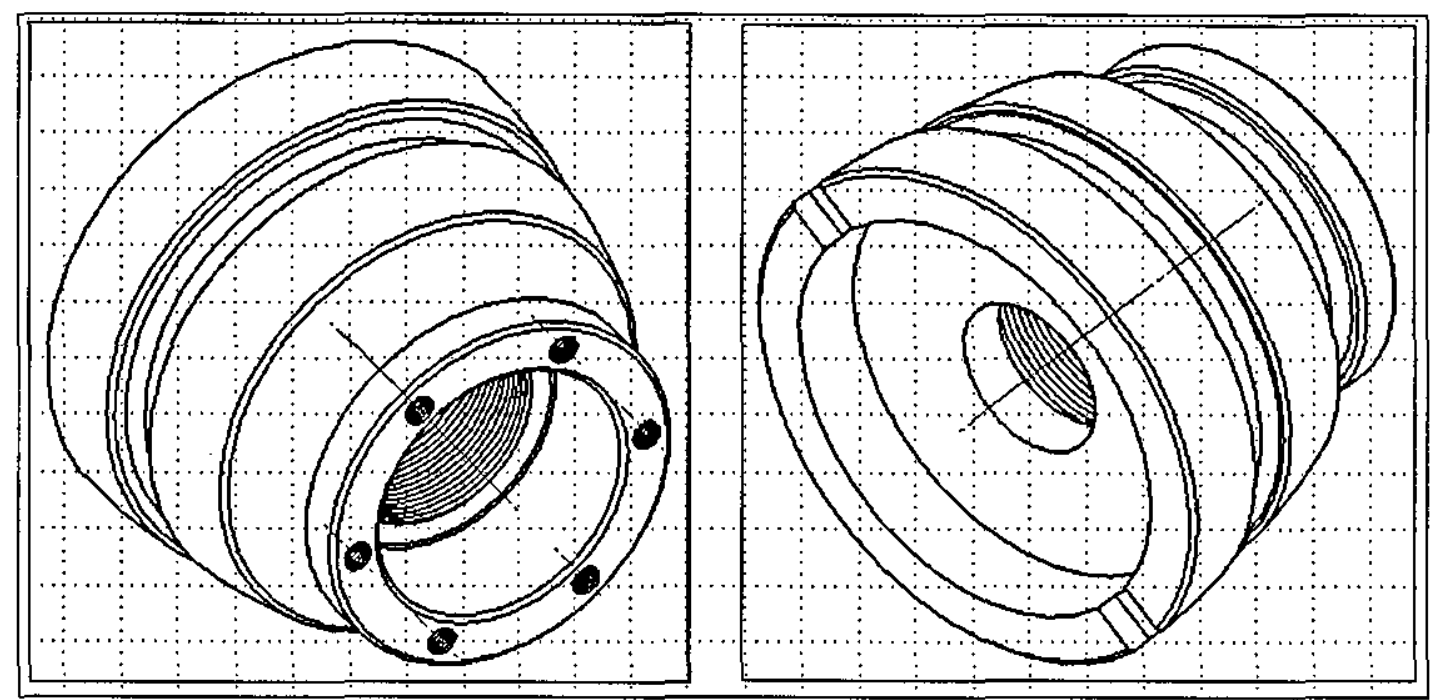

Figure 7.19 3D drawing for component no 2

Table 7.4 Component 2 descriptions

\begin{tabular}{|c|c|c|c|}
\hline \multirow{2}{*}{$\begin{array}{l}\text { General } \\
\text { Attributes }\end{array}$} & \multicolumn{2}{|c|}{ Feature Details } & \multirow{2}{*}{$\begin{array}{l}\text { Machining } \\
\text { operations }\end{array}$} \\
\hline & OD Profile & ID Profile & \\
\hline Double sided & Surface straight & Straight & Turning \\
\hline Asymmetrical & Slight undercut arc & $\begin{array}{l}\text { Various radius } \\
\text { corners }\end{array}$ & Grooving \\
\hline $\begin{array}{l}\text { At least } 3 \text { axis } \\
\text { lathe required }\end{array}$ & Taper straight & Small radius corner & Drilling \\
\hline $\mathrm{X}, \mathrm{z}$ and $\mathrm{c}$ axis & Rounded groove & Hole & Mill on side face \\
\hline
\end{tabular}

As mentioned before this component is based on a Mazak component and a detailed operation plan based on a conventional approach is shown in table 7.5. and detailed in Appendix $\mathbf{H}$, to give an idea of the technical data that must be considered to produce this component. 


\begin{tabular}{|c|c|c|c|c|c|c|}
\hline & \multicolumn{5}{|c|}{ Table 7.5 Operation Plan for Component 2} & \\
\hline $\begin{array}{l}\text { Op. } \\
\text { No }\end{array}$ & $\begin{array}{l}\text { Op. } \\
\text { Type }\end{array}$ & Op. Description & $\begin{array}{l}\text { Tool } \\
\text { No. }\end{array}$ & $\begin{array}{c}\text { Tool } \\
\text { description }\end{array}$ & $\begin{array}{l}\text { Spindle } \\
\text { speed }\end{array}$ & $\begin{array}{c}\text { Cutting } \\
\text { feed }\end{array}$ \\
\hline 1 & Turn & Face the billet & $\mathrm{T} 1$ & Facing tool & 3600 & 0.2 \\
\hline 2 & Turn & $\begin{array}{l}\text { Rough the external } \\
\text { profile }\end{array}$ & $\mathrm{T} 2$ & $\begin{array}{l}\text { External } \\
\text { turning tool }\end{array}$ & 3600 & 0.2 \\
\hline
\end{tabular}

\subsubsection{Operations by SCSTO}

The main purpose of this case study is to investigate the prototype system's capability of creating features directly from a CAD file. The case study component has been designed in Unigraphics software, exported as a STEP AP 203 file and then imported into SCSTO. Case study component 2 has a minimum of one set-up if the machine has a counter spindle and two-sided machining. The complete machining process involves twenty three processes and also depends on the machine configuration as shown in figure 7.20. The figure clearly shows this type of machining should have a linear axis and a rotary axis (c axis). The overall process is similar to case study component 1 , but the number of features and manufacturing is different. This demonstration for case study component 2 , starts with the user beginning the modelling process by selecting the base part as the cylinder type. This shape is considered as the starting raw material for modelling the component. Then the user creates a step feature and attaches it to the base part. The remaining features are created and attached to the base part. The component was created in Unigraphics (UG) version NX3 as shown in figure 7.21 and Figure 7.22 shows detail processes to export file based on UG environment. 
Chapter 7

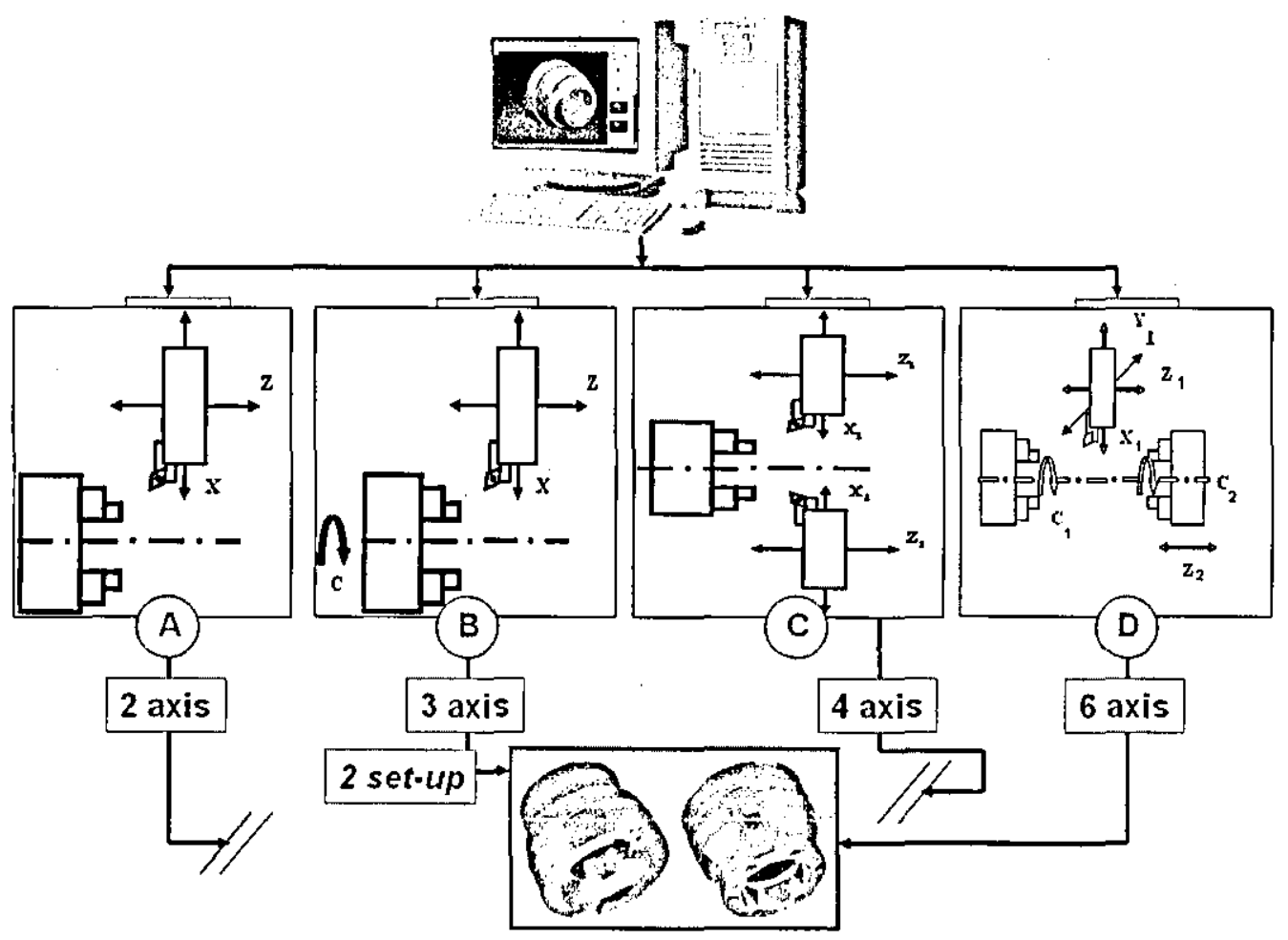

Figure 7.20 the Process planning for case study component 2

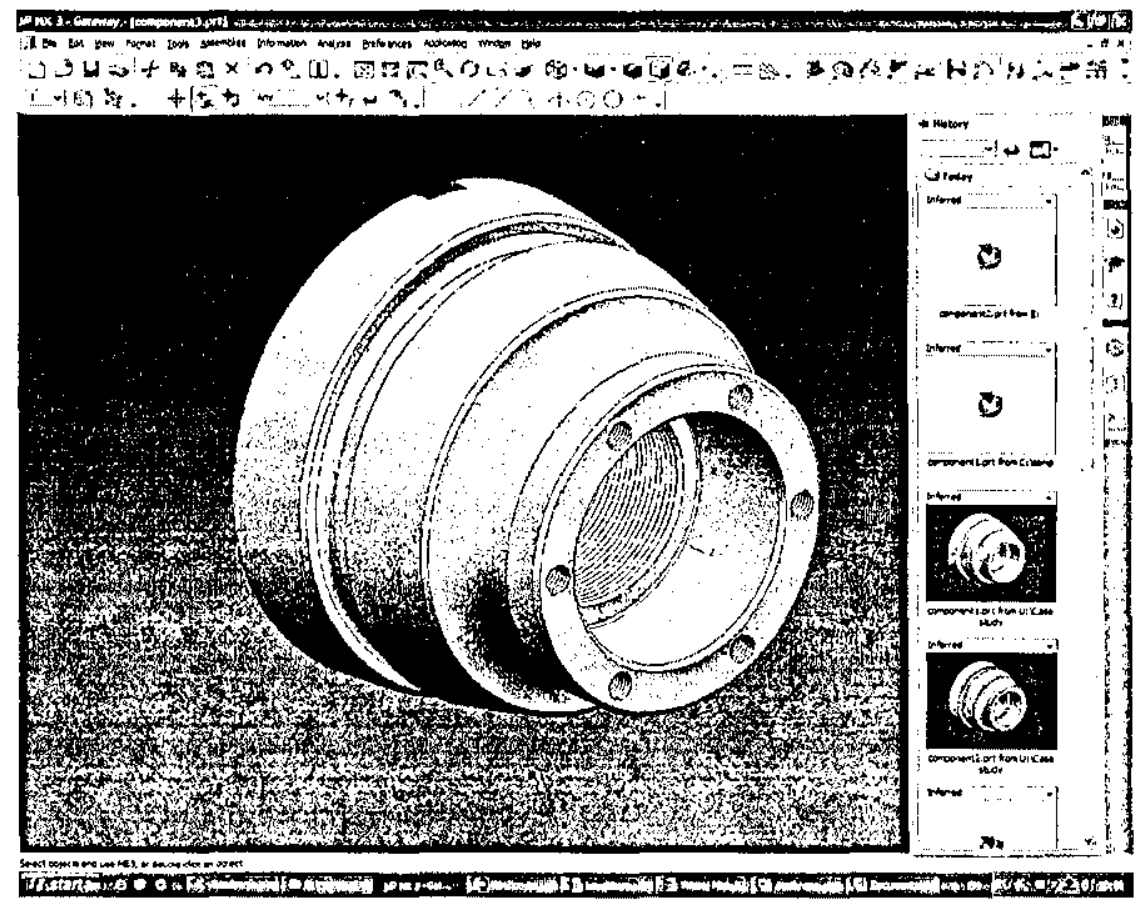

Figure 7.21 the Unigraphics design for component 2 


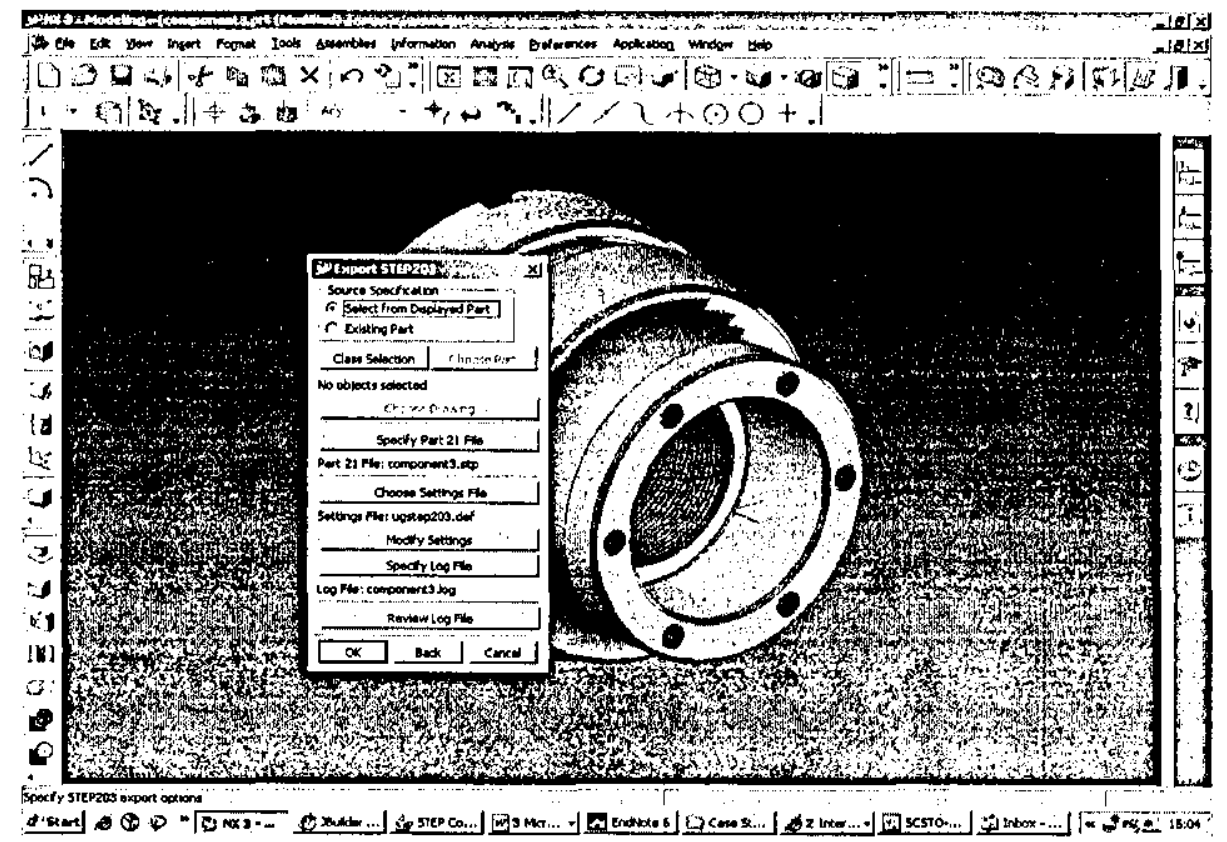

Figure 7.22 export file for component 2

The process for creating features starts with importing the CAD file into SCSTO from the main menu as shown in figure 7.23 The CAD file in this case is an AP 203 file. 


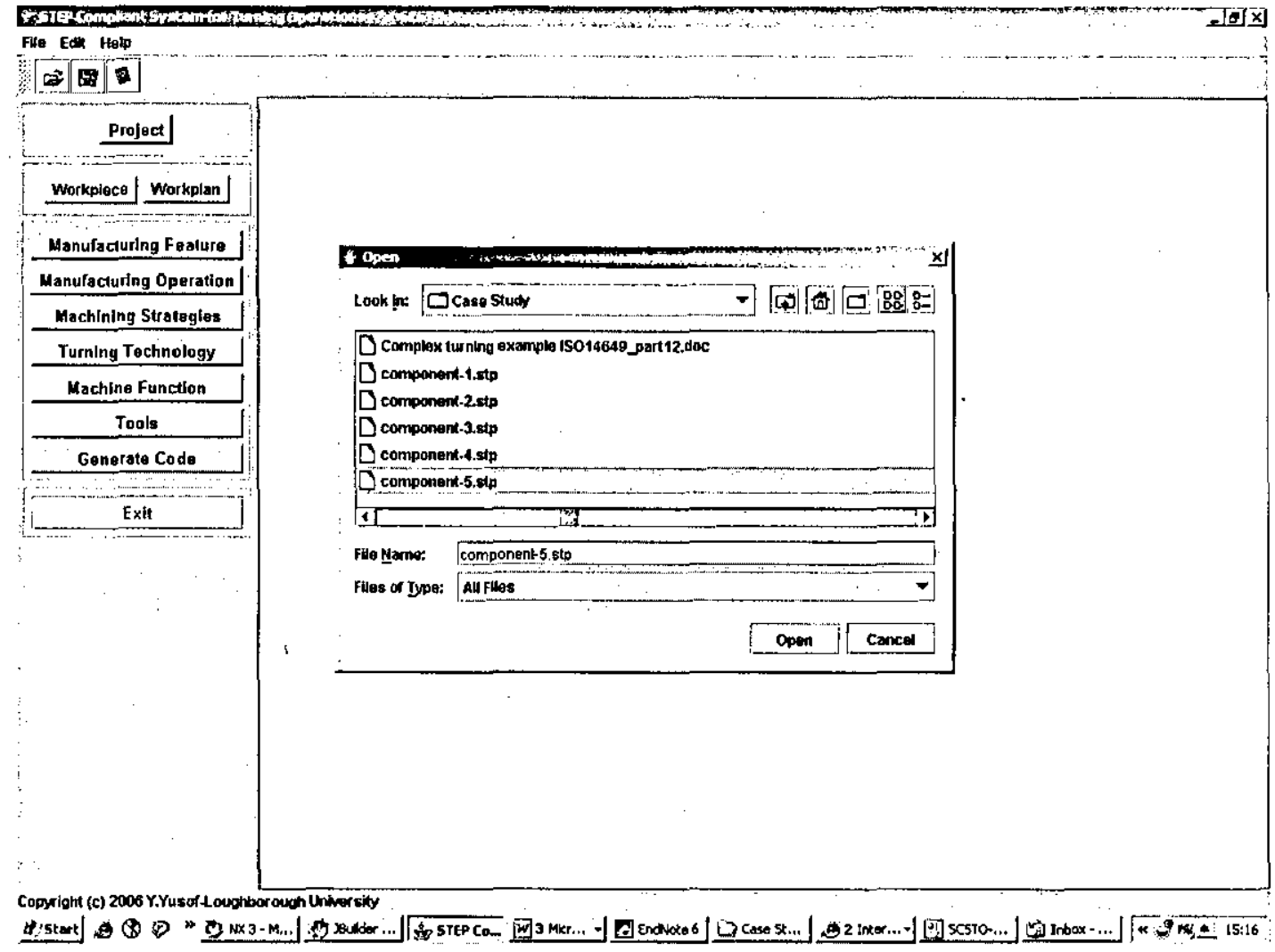

Figure 7.23 open file menu

After the file has been taken as the input file format, Boundary Representation (BRep) is converted into STEP data using the Parasolid Application Protocol Interface (API). Then the features need to be located and oriented to the base part. The user can check the features and can create Boolean operations as mentioned in component 1 . The difference from component 1 is the way of creating the features, and the subsequent processes are the same. Figure 7.24 shows the manufacturing process for this component. 


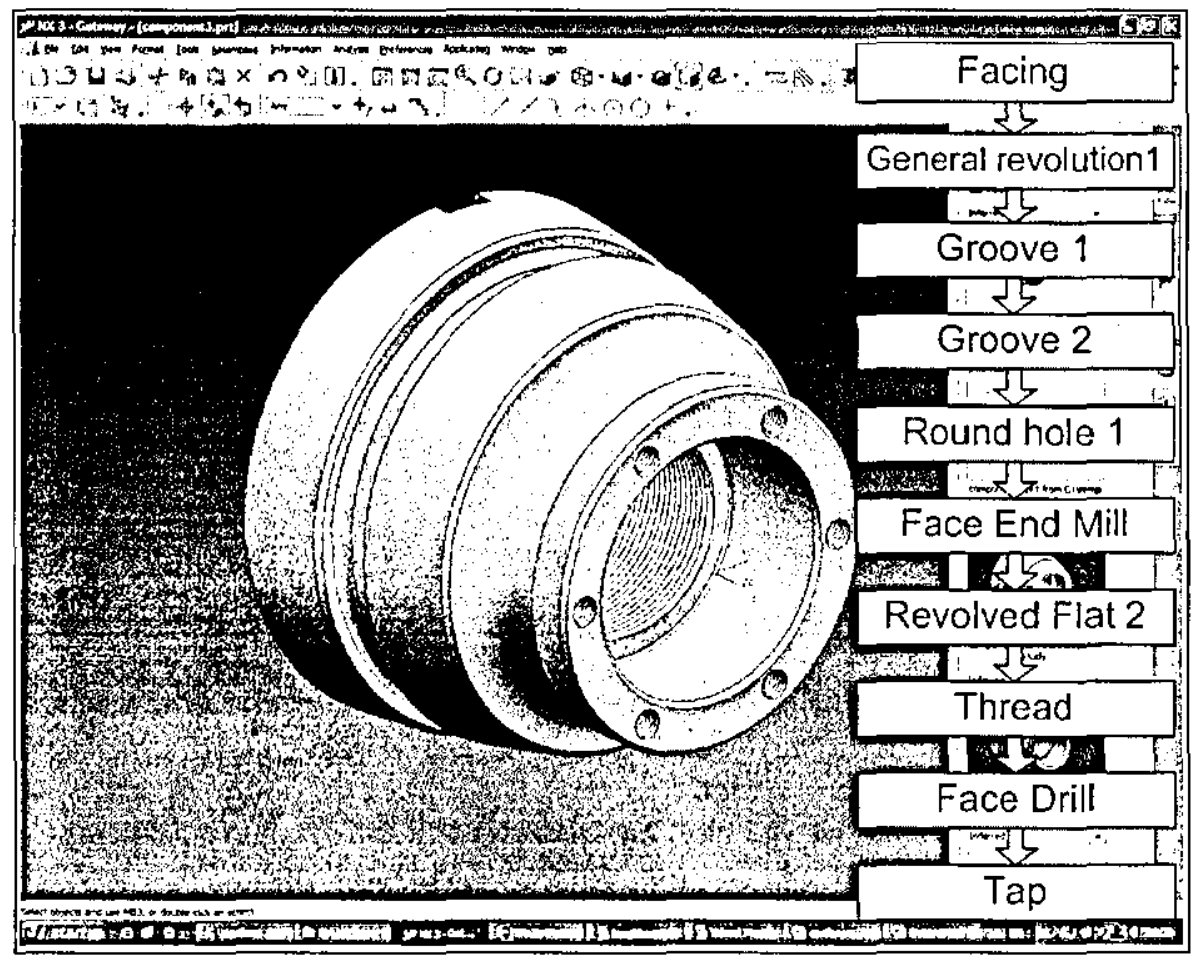

Figure 7.24 Manufacturing process of component 2

After recognising the features, they need to be located on the base part as shown in figure 7.25

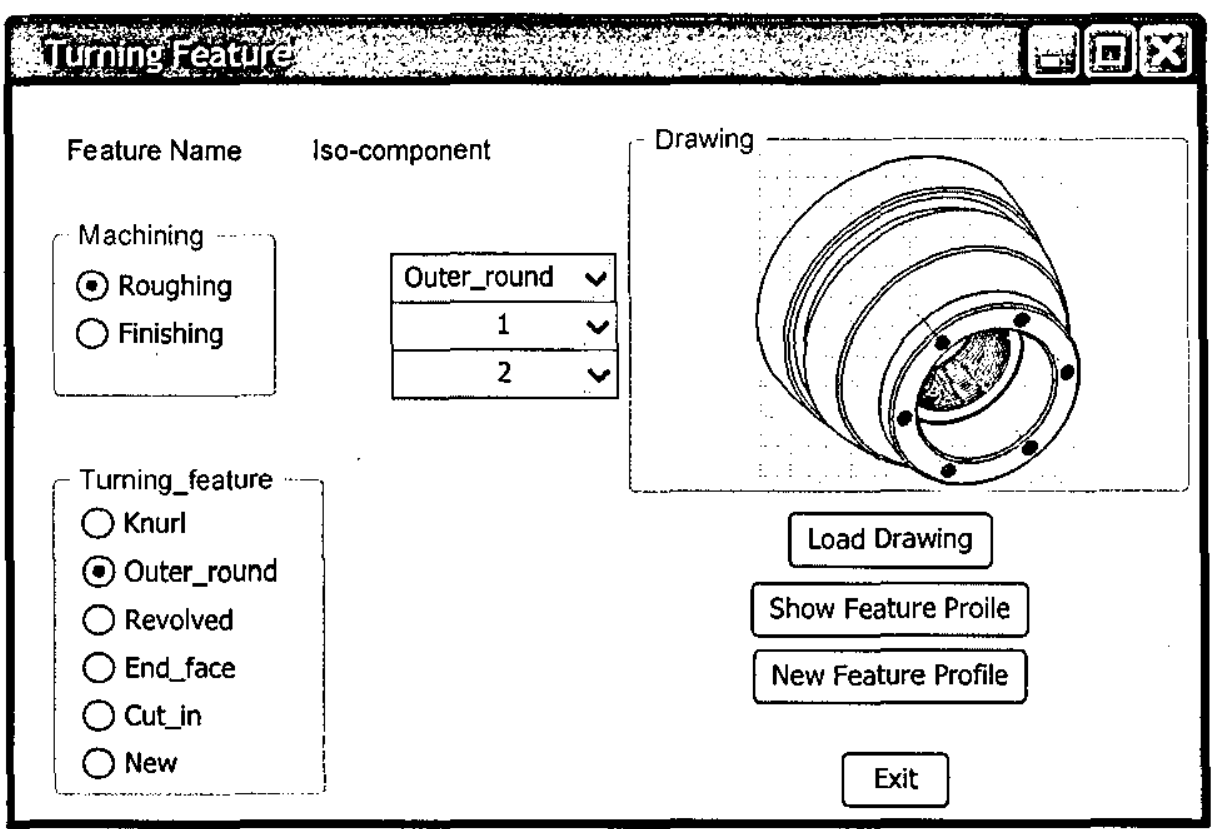

Figure 7.25 Turning feature of case study component 2 
Figure 7.26 depicts the dialog that appears just after the workplan has been created.

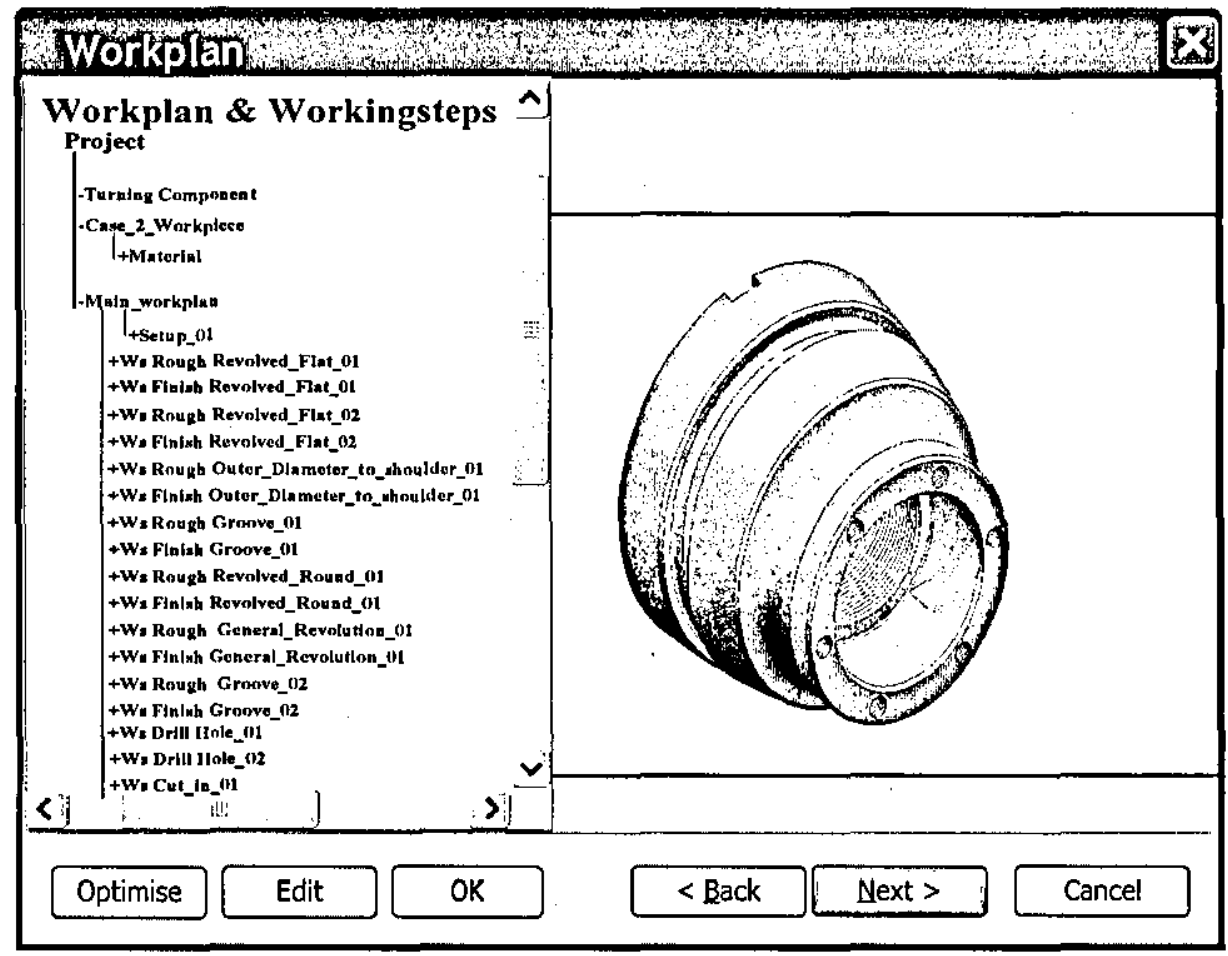

Figure 7.26 workplan and workingstep for component 2

The manufactured product after the finishing process is shown in figures 7.27 and 7.28 .

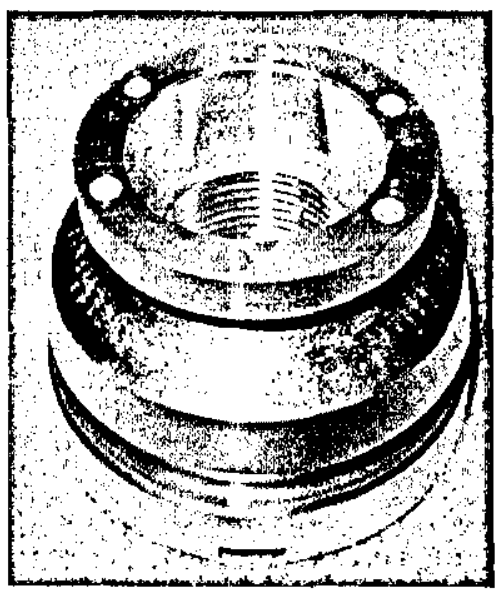

Figure 7.27 Final product (top-view) for component 2 


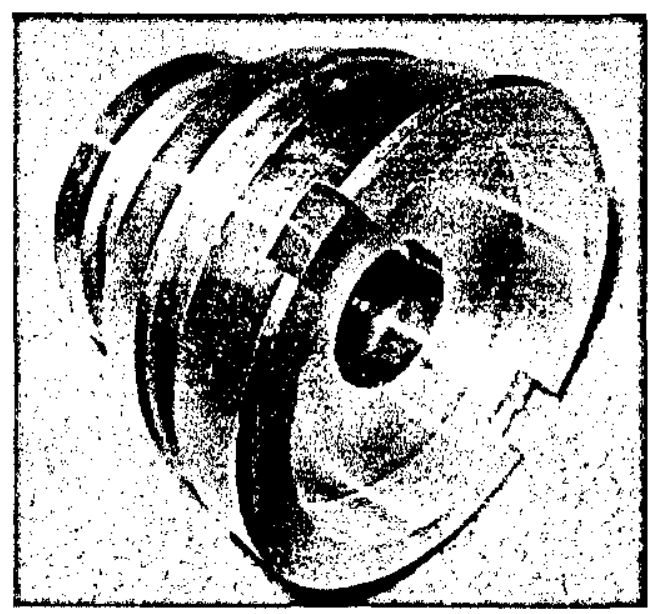

Figure 7.28 Final product (side-view) for component 2

\subsection{Summary}

This chapter has described a prototype system to validate the functioning of SCSTO. The input to SCSTO is the geometrical parameters of the workpiece and its features. The case studies have demonstrated the SCSTO capability of assisting in generating process plans for turning operations with geometry either defined by users or generated from an AP 203 CAD file. Case study 1 successfully generated the physical file and compliant with STEP-NC. Case study 2 demonstrated that features direct from CAD file is a better approach especially for complex components. The case studies have shown this prototype system can work either by the user creating features or by importing from a CAD file. Overall it has been shown that this prototype system has good opportunities to generate the part program compliant with ISO 14649. 


\section{Chapter 8-Conclusion and Recommendations}

\subsection{Introduction}

This chapter presents an overview and brief discussion of the research work that has been presented in this thesis. The discussion provides a summary of all the previous research conclusions and research contributions. Finally some recommendations for future research are given.

\subsection{Thesis Summary}

This thesis focuses on the use of the new standard; ISO 14649 (STEP-NC), to address the process planning and machining of rotational components. The principle motivation which led to this research was a desire to investigate how recent developments in standards might influence the product life-cycle in a global manufacturing environment. Consequently, the principle aim was to explore the use of the STEP-NC standard in the machining of asymmetrical parts in an interoperable manufacturing context. Interoperability refers in this instance to the ability to share information between the CAD and CAM phases of the life-cycle and to do this independent of machine tool equipment and location. This led directly to the main objectives which included the creation of a STEP-NC compliant information model and its embodiment in a prototype computer system. A literature review which focused on ISO and the standards for information exchange included the current status of STEP-NC (ISO 14649) documents and confirmed its potential suitability for this research. A review of research literature focused on STEP-Compliant process planning and manufacturing and discussed 
research work related to STEP-NC. This review established that milling had been the primary concern of earlier work and that turning was relatively unconsidered. However, ideas established in this earlier research were incorporated in this work, especially the use of formal information models, the importance of bi-directional flows of information between $\mathrm{CAD}$ and $\mathrm{CAM}$ and the potential of feature extraction. A design and specification for a prototype computer system that was developed on the basis of these reviews and on the idea that STEP information models should be available in universal formats familiar to most business application developers: specifically XML and the Unified Modelling Language (UML) were used to demonstrate the feasibility of the approach and case studies were developed involving two components based on an ISO 14649 Part 12 example and a component that showed increasing levels of complexity from standard turning to multi-axis machining.

\subsection{Research Contributions}

The main objective of this research work was to explore the application of new data standards for CNC machining for asymmetrical parts to enable data exchange of "manufacturing rich" information between CAD and CNC to support interoperable manufacture during the product development process. These objectives are realized through the following contributions.

i. The fuller understanding of how the STEP-NC standards might contribute to the goal of interoperability. At the heart of interoperability is the ability to seamlessly transfer manufacturing information to any machine that has the required manufacturing capability and a STEP-NC compliant controller.

ii. The design of a STEP-NC compliant CAx system framework and information models for turning operations compliant with ISO 14649 Part 12. Previous work has almost exclusively been concerned with milling with 
some work in others area such as EDM. The work reported here is the most significant in the field of turning.

iii. Extension of the above STEP-NC compliant system framework and information models for machining operations compliant with both Parts 11 and 12 of ISO 14649. This is an important demonstration of how parts 11 and 12 of the STEP-NC can be combined to describe the manufacture of complex turn/mill parts on sophisticated and highly capable machining centres.

iv. A prototype system which can be used for further experimentation and perhaps as the basis for an eventual commercial implementation. Such an implementation of a STEP-NC based CNC system would give another alternative to the conventional CNC systems based on the old ISO 6983 standard which have been used for the last few decades.

\subsection{Recommendation for Future Research}

In order to meet the requirements for global manufacturing of real-life parts, several extensions will be needed to this research including;

i. Make it fully automatic so the user simply loads files generated from a CAD system using files such as STEP AP203 or AP214 as input. A feature modeller needs to be fully implemented into the prototype system in order to import files directly from a CAD system and automatically generate the process plan. The feature modeller and feature library can work together to make sure all the features that have been defined by STEP 224 can be created for complex parts. Finally the process plan generator will generate the part program and send it to the machines. Automatic transfer of design information into the manufacturing system is an essential component of an effective bi-directional transfer of data. There would of course be much 
work in transferring information back to design from manufacturing, but it is to be hoped that STEP-NC and the wider STEP standard would make this a real possibility.

ii. As process planning is a rule-based activity intelligent methods could be applied to SCSTO. In terms of complexity of the component, an expert system may be the best approach to machine the turn/mill component. These techniques will minimise the human interface to the system and also minimise the errors or mistakes made by users. Further research needs to consider the implementation of an expert system into the SCSTO architecture in order to cover a wider range of manufacturing aspects including for example inspection of the finished part which might be permit quality control to be done during the machining processes.

iii. Apply the approach for turn/mill components with increasing number of axes to match the capabilities of modern machines that can have more than five axes. This research work focuses on individual rotational parts with limited machine axes and needs to be extended to include complex features with combinations of milling and turning features.

iv. There is a need to provide support for collaborative design and manufacturing planning that might take place on a global basis. A possible method for achieving this is to arrange for output files in XML format because XML files can be supported by internet interfaces so can be easily used to query and update data in the database over the internet for emanufacturing. A lot of research focuses on e-manufacturing and will increasingly use the XML format. The e-manufacturing will be one of the best solutions for global manufacturing because it will realise the concept of DABA or "Design Anywhere, Build Anywhere".

v. Other life cycle activities. The most obvious activity to extend to is 
the Production Planning area. i.e. including capacity and sequencing issues in manufacturing planning and base this on part descriptions which include STEP-NC knowledge. The point here is that if the manufacturing instructions are not machine-dependent (because they are in STEP-NC) then some of the constraints as to which machine is used would be removed from Production Planning.

\subsection{Conclusion}

This research work was motivated by industrial requirements of concurrent engineering, standard product data models and an integrated manufacturing environment, which are further reflected in process planning. The conclusions of the work can be elaborated as:

i. The information modelling in this area addresses the problem of capturing and representing manufacturing information related to resources and processes. The interaction between the different types of models could provide a description of the products, how they should be manufactured, and what manufacturing resources should be used. This would provide an information platform upon which several different computer-based tools to support the innovation process can be built. This will allow the provision of reliable manufacturing information to assist in the performance of product development life cycle activities and related decisions.

ii. SCSTO was developed to generate a Part 21 file based on machining features to support the interactive generation of process plans utilising feature extraction. The system was constructed using a structured methodology for its planning and object-oriented methods for its implementation. 
iii. A case study component was tested to show that the new approach (STEP-NC) can generate code which is comparable to the currently used Gcode with some benefits such as the elimination of the post-processor.

iv. Efforts are under way to fulfil the STEP-NC challenge by combining Parts 11 and 12, for turn/mill operations. STEP-NC forms a possible basis to satisfy the latest requirements and demands with respect to a bi-directional CAx process chain for machining.

v. STEP-NC forms a possible basis to satisfy the latest requirements and demands with respect to a bi-directional CAx process chain for machining. In addition its development as a future manufacturing platform to enable different process models to be integrated for the adaptable integration of $\mathrm{CAD} / \mathrm{CAPP} / \mathrm{CAM}$ and $\mathrm{CNC}$ will be a major avenue of research for years to come.

It is expected that the recommended future extensions will enhance the usefulness of this research, and will meet the requirements for global interoperable manufacturing for real-life parts. 


\section{References}

Ahlquist, F. (2002). "A Methodology for Operation Planning," Licentiate thesis, Lund University, Lund, Sweden.

AIAG. (1997). "Automotive Industries Action Group (AIAG), Measurement Systems Analysis, Second edition,." AIAG, Southfield, MI, USA.

Ali, L. (2005). "Development of a STEP-NC Compliant Feature-based Inspection Framework for Prismatic Parts," Doctoral Thesis, Loughborough University, Loughborough.

Ali, L., Newman, S. T., and Petzing, J. N. (2005). "Development of a STEPCompliant inspection framework for discrete components." Proceedings of the Institution of Mechanical Engineers, Part B: Journal of Engineering Manufacture, 219, July 2005, 557-563.

Allen, R. D. (2003). "An Agent Based Approach to STEP-NC CAM/CAM," Doctoral Thesis, Loughborough University, Loughborough.

Allen, R. D., Harding, J. A., and Newman, S. T. (2005). "The application of STEPNC using agent-based process planning." International Journal of Production Research, 43(4), 655-670.

Allen, R. D., Newman, S. T., Harding, J. A., and Rosso-Jr., R. S. U. (2003) "The Design of a STEP-NC Compliant Agent Based CAD/CAM System." Proceedings of the 13th International Conference on Flexible Automation and Intelligent Manufacturing Conference (FAIM2003), Tampa, Florida, USA., 530-540. 
Alting, L., and Zhang, H. (1989). "Computer aided process planning: A state of the art survey." International Journal of Production Research, 27(4), 553-585.

Amaitik, S., and Kilic. (2007). "An intelligent process planning system for prismatic parts using STEP features." The International Journal of Advanced Manufacturing Technology, 31(9-10), 978-993.

Amaitik, S. M. (2005). "Development of A Step Feature-Based Intelligent Process Planning System For Prismatic Parts," Doctoral Thesis, Middle East Technical University, Ankara, Turkey.

Aslan, E., Seker, U., and Alpdemir, N. (1999). "Data extraction from CAD model for rotational parts to be machined at turning centres." Turkish Journal of Engineering and Environmental Sciences, 23(5), 339-347.

Baymgarter, H. (1998). "A STEP to Improved CAD." Mechanical Engineering, $120(2), 84-85$.

Bhandarkar, M. P., Downie, B., Hardwick, M., and Nagi, R. (2000). "Migrating from IGES to STEP: One to one translation of IGES drawing to STEP drafting data." Computers in Industry, 41(3), 261-277.

Bhandarkar, M. P., and Nagi, R. (1997). "STEP Product information models in agile manufacturing." International Journal of Agile Manufacturing, 1, 6575.

Booch, G., Rumbaugh, J. and Jacobson, I.,. (1999). The Unified Modelling Language User Guide, , Addison-Wesley; ACM Press, Reading, MA.

Borland. (1997). Developer's Guide - JDataStore 7, Borland Software Corporation, Scotts Valley, California. 
Brecher, C., Vilr, M., and Wolf, J. (2006). "Closed-Loop CAPP/CAM/CNC Process Chain Based on STEP and STEP-NC Inspection Tasks." International Journal of Computer Integrated Manufacturing, 19(6), 570-580.

Case, K., and Harun, W. A. R. W. (2000). "Feature-based representation for manufacturing planning." International Journal of Production Research, $38(17), 4285-4300$.

Chen, P. P. (1976). "The entity-relationship model: toward a unified view of data." ACM Transactions on Database Systems, 1(1), 9-36.

Chep, A., and Tricarico, L. (1999). "Object-oriented analysis and design of a manufacturing feature representation." International Journal of Production Research, 37(10), 2349-2376.

Choi, I., Suh, S.-H., Kim, K., Song, M., Jang, M., and Lee, B.-E. (2006). "Development process and data management of TurnSTEP: a STEPcompliant CNC system for turning." International Journal of Computer Integrated Manufacturing, 19(6), 546-558.

Dong, J. J., Parsaei, H. R., and Leep, H. R. (1996). "Manufacturing process planning in a concurrent design and manufacturing environment." Computer \& Industrial Engineering, 30(1), 83-93.

Dutta, D., Kumar, V., Pratt, M. J., and Sriram, R. (1998) "Towards STEP-based data transfer in layered manufacturing." the tenth international IFIP WG5.2/5.3 PROLAMAT Conference, Trento, Italy.

Erdos, G., and Xirouchakis, P. (2003) "STEP-NC Data Model Development For Wire-EDM Manufacturing." 7th IFAC Workshop on Intelligent manufacturing Systems, 2003., Budapest, Hungary. 
ESPRIT. (1997). "Project 8643, Optimised preparation of manufacturing information with multi-level CAM-CNC coupling (OPTIMAL): final report for publication."

Feeney, A. B., and Frechette, S. (2002) "Testing STEP-NC Implementations." Proceedings of the 5th Biannual, World Automation Congress, 39-44.

Fidan, T. (2004). "Feature based design of rotational parts based on STEP," Doctoral Thesis, Middle East Technical University, Ankara, Turkey.

Fortin, E., Chatelain, J. F., and Rivest, L. (2001) "Improving Information Flow from CAM to CNC : an Innovative Architecture Based on BNCL Virtual Machine." Computer \& Industrial Engineering International Conference (29th ICC\&IE), Montreal, Canada.

Gallaher, M. P., and O'Connor, A. C. (2002). "Economic Impact Assessment of the International Standard for the Exchange of Product Model Data (STEP) in Transportation Equipment Industries." National Institute of Standards and Technology, Gaithersburg, US. 193.

Giachetti, R. E. (1999). "A standard manufacturing information model to support design for manufacturing in virtual enterprises." Journal of Intelligent Manufacturing, 10, 49-60.

Glantschnig, F. (2001) "International Activities for STEP and STEP-NC Manufacturing Standards." EMO 2001, Hanover, Canada.

Goldstein, B., Kemmerer, S., and Curts, P. (1998). "A Brief History of Early Product Data Exchange Standards - NISTIR 6621." National Institute of Standards and Technology (NIST), Gaithersburg, MD. 1-17. 
Gu, P., and Norrie, D. H. (1995). Intelligent Manufacturing Planning, Chapman \& Hall, New York.

Hardwick, M. (2002). "Digital manufacturing using STEP-NC." Technical Paper Society of Manufacturing Engineers. MS(MS02-252), 12.

Hardwick, M. (2003). "STEP into Automatic Machining - Manufacturing moves toward new frontiers." STEP Tools - STEP-NC white paper.

Hardwick, M., and Loffredo, D. (2006). "Lessons Learned Implementing STEP-NC AP238." International Journal of Computer Integrated Manufacturing, $19(6), 523-532$.

Heusinger, S. (2001). "ISO 14649 STEP-NC; Data model and implementation for turning in Germany - presentation material." WG7 meeting, Frankfurt.

Heusinger, S. (2003). "Data model and implementation for turning in Germany presentation material." Aachen University, Aachen, Germany.

Heusinger, S., Rosso-Jr, R. S. U., Klemm, P., Newman, S. T., and Rahimifard, S. (2006). "Integrating the CAx Process Chain for STEP-Compliant NC Manufacturing of Asymmetric Parts." International Journal of Computer Integrated Manufacturing, 19(6), 533-545.

Ho, K. H., Newman, S. T., and Allen, R. D. (2005). "STEP-NC compliant information modelling for wire electrical discharge machining component manufacture." Proceedings of the Institution of Mechanical Engineers. Part B, Journal of Engineering Manufacture, 209(10), 777 -784. 
Huang, H. M., Kramer, T. R., Messina, E., Proctor, F. M., and Scott, H. A. (2001). "A Feature-Based Inspection and Machining System." Computer Aided Design, 33, 653-669.

Hwang, H.-J., Han, S., and Kim, Y.-D. (2004). "Mapping 2D midship drawings into a 3D ship hull model based on STEP AP218." Computer Aided Design, 36, 537-547.

ISO. (1982). "International Organization for Standardisation, ISO 6983-1:1982, Numerical control of machines - Program format and definition of address words - Part 1: Data format for positioning, line motion and contouring control systems."

ISO. (1994). "International Organization for Standardisation - ISO 10303-1:1994, Part 1: Overview and fundamental principles. Product data representation and exchange."

ISO. (2000a). "International Organization for Standardisation - ISO/IS 10303-224 Product data representation and exchange: Application protocol: Mechanical product definition for process planning using machining features."

ISO. (2000b). "International Organization for Standardisation - ISO/IS 10303-224.2, Product data representation and exchange: Application protocol: Mechanical product definition for process planning using machining features."

ISO. (2002). "International Organization for Standardisation - ISO 10303-Part 21 Industrial automation systems and integration - Product data representation and exchange - Part 21: Implementation methods: Clear text encoding of the exchange structure." 
ISO. (2003a). "Industrial automation systems and integration - Product data representation and exchange - Part 214: Application protocol: Core data for automotive mechanical design processes."

ISO. (2003b). "International Standard 14649-1: Part 1 : Industrial automation system and integration - Physical device control - Data model for computerised numerical controllers - Part 1: Overview and fundamental principles."

ISO. (2004a). "International Standard 14649-10: Part 10 : Industrial automation system and integration - Physical device control - Data model for computerised numerical controllers - Part 10 : General process data."

ISO. (2004b). "International Standard 14649-11: Part 11 : Industrial automation system and integration - Physical device control - Data model for computerised numerical controllers - Part 11 : Process data for milling."

ISO. (2004c). "ISO 10303-11 Industrial Automation Systems and Integration Product Data Representation and Exchange - Part 11: Description Methods: the EXPRESS Language Reference Manual."

ISO. (2005a). "International Standard 14649-12: Part 12 : Industrial automation system and integration - Physical device control - Data model for computerised numerical controllers - Part 12 : Process data for turning."

ISO. (2005b). "International Standard 14649-121: Part 121 : Industrial automation system and integration - Physical device control - Data model for computerised numerical controllers -Part 121: Tools for Turning Machines." 
ISO/FDIS. (2004). "Industrial automation systems and integration - Physical device control - Data model for computerized numerical controllers - Part 111: Tools for milling machines."

Kemmerer, S. J. (1999). STEP The Grand Experiences, NIST Special Publication 939, Gaithersburg,.

Kim, M., J.Noh, S., Kim, Y., and Kang, S.-H. (2002). "A customizable view for Web-based STEP data." Data \& Knowledge Engineering, 43, 29-56.

Kramer, T. R., Proctor, F. M., Xu, X. W., and Michaloski, J. L. (2006). "Run-time interpretation of STEP-NC: Implementation and performance." International Journal of Computer Integrated Manufacturing, 19(6), 495507.

Kumar, S., Nassehi, A., Newman, S. T., Allen, R. D., and Tiwari, M. K. (2007). "Process control in CNC manufacturing for discrete components: A STEPNC compliant framework." Robotics and Computer-Integrated Manufacturing.

Landy, M., Siddiqui, S., and Swisher, J. (2003). JBuilder Developer's Guide.

Larsson, M. (2006). "Manufacturing System Data Management and Development Towards a STEP compliant reference model for manufacturing," Licentiate Thesis, Royal Institute of Technology (KTH), Stockholm, Sweden.

Lee, J. Y., and Kim, K. (1998). "A feature-based approach to extracting machining features." Computer Aided Design, 30(13), 1019-1035. 
Lee, W., and Bang, Y. B. (2002). "PC Based STEP-NC Milling Machine Operated by STEP-NC in XML Format." Journal- Korean Society of Precision Engineering, 19(12), 185-193.

Lee, W., and Bang, Y.-B. (2003). "Design and implementation of an ISO14649compliant CNC milling machine." International Journal of Production Research, 41(13), 3007-3017.

Lee, W., Bang, Y. B., Ryou, M. S., Kwon, W. H., and Jee, H. (2006). "Development of a PC-Based Milling Machine Operated by STEP-NC in XML Format." International Journal of Computer Integrated Manufacturing, 19(6), 593-602.

Lewis, J. (2002). "One STEP closer for digital manufacturing. By burying G-codes and post-processors within compilers, STEP-NC stands poised to streamline machine tool programming." Design News, 57(20), 44-46.

Li, R.-K. (1988). "A part-feature recognition system for rotational parts." International Journal of Production Research, 26(9), 1451-1475.

Lin, A. C., Lin, S. Y., and Cheng, S. B. (1997). "Extraction of manufacturing features from a feature-based design model." International Journal of Production Research, 35(12), 3249-3288.

Liu, R., and Zhang, C. (2004) "An Agent Based Framework for The Intelligent STEP-NC Controller." The 6th International Conference on Frontiers of Design and Manufacturing (ICFDM 2004), X'ian, China.

Liu, R., Zhang, C., Zhang, Y., and Wang, R. (2004a). "On CNC Machining and Part Program Pre-processing Based on STEP-NC." Materials Science Forum, 471-472, 326-329. 
Liu, R., Zhang, C., Zhang, Y., and Wang, R. (2004b). "Process planning model and heuristics for CNC machining based on STEP-NC Source." Zhongguo Jixie Gongcheng/China Mechanical Engineering, 15(4), 325-329.

Liu, R., Zhang, C. R., Nassehi, A., and Newman, S. T. (2007). "A STEP-NC programming system for prismatic parts." Materials Science Forum, 532533, 1108-1111.

Liu, R. L., C. R. Zhang. (2004). "Solutions for Interpreting STEP-NC Based Part Program." Materials Science Forum, 471-472, 344-347.

Maeder, W., Nguyen, V. K., Richard, J., and Stark, J. (2002) "Standardisation of the Manufacturing Process: The IMS STEP-NC Project." IPL (National Network of Competence on Integrated Production and Logistics) Net Workshop, Saas Fee, Switzerland.

Mazak. (2002). "CNC System Mazatrol T Plus Technical Specifications." Nagoya, Japan. 34.

Michaloski, J. (2005) "Case Study in the Challenges of Integrating CNC Production and Enterprise Systems." ISA EXPO, Chicago, Illinois, US.

Molina, G. (1995). "A Manufacturing Model to Support Data Driven Applications for design and manufacture," Doctoral Thesis, Loughborough University, Loughborough.

Motavalli, S., Cheraghi, S. H., and Shamsaasef, R. (1997) "Feature-based modelling; An object oriented approach." Proceedings of the 21st International Conference on Computers and Industrial Engineering, 349. 352. 
Muller, P. (2000a). "STEP-NC - New data interface for NC programming." STEPNC Newsletter, Issue 1, April 2000, Erlangen, Germany, 1-2.

Muller, P. (2000b). "STEP-NC - New data interface for NC programming." STEPNC Newsletter, Issue 2, July 2000, Erlangen, Germany, 1-4.

Muller, P. (2000c). "STEP-NC - New data interface for NC programming." STEPNC Newsletter, Issue 3, November 2000, Erlangen, Germany, 1-9.

Muller, P. (2003). "STEP-NC - New data interface for NC programming." STEPNC Newsletter, Issue 5, September 2003, Erlangen, Germany, 1-5.

Muller, P. (2004). "STEP-compliant Data Interface for Numerical Controls - Final Technical Report." GRD1-2001-00007, IMS STEP-NC Consortium, Erlangen, Germany. 1-41.

Nassehi, A., Allen, R. D., and Newman, S. T. (2006a) "Intelligent Replication of Manufacturing Information between CAD/CAM Systems and CNC Controllers." Proceedings of the 16th International Conference on Flexible Automation and Intelligent Manufacturing Conference (FAIM2006), Limerick, Ireland, June 2006, 413-420.

Nassehi, A., Newman, S. T., and Allen, R. D. (2005) "The application of multiagent systems in STEP-NC compliant process planning of prismatic components." Proceedings of the 15th International Conference on Flexible Automation and Intelligent Manufacturing Conference (FAIM2005), Bilbao, Spain, 202-209. 
Nassehi, A., Newman, S. T., and Allen, R. D. (2006b). "The application of multiagent systems for STEP-NC computer aided process planning of prismatic components." International Journal of Machine Tools \& Manufacture, $46(5), 559-574$.

Nassehi, A., Newman, S. T., and Allen, R. D. (2006c). "STEP-NC compliant process planning as an enabler for adaptive global manufacturing." Robotic and Computer Integrated Manufacturing, 22(5-6), 456-467.

Newman, S. (2004a). "Integrated manufacture for the 21 st century Development of the STEP-NC standard and its implications for manufacturing processes worldwide." Metalworking Production, 148(6), 13-16.

Newman, S., Harding, J., Young, B., Petzing, J., Rahimifard, S., and Case, K. (2004). "Intelligent Manufacture for STEP-NC Compliant Machining and Inspection - EPSRC Research Proposal." Loughborough University. 1-45.

Newman, S. T. (2004b) "Integrated CAD/CAPP/CAM/CNC Manufacture for the 21st century." International Conference in Flexible Automation and Intelligent Manufacture (FAIM2004), Toronto, Canada.

Newman, S. T., Allen, R. D., and Rosso-Jr, R. S. U. (2002) "CAD/CAM solutions for STEP Compliant CNC Manufacture." Proceedings of the 1st CIRP(UK) Seminar on Digital Enterprise Technology, School of Engineering, University of Durham.

Newman, S. T., Nassehi, A., Xu, X. W., Rosso-Jr, R. S. U., Wang, L., Yusof, Y., Ali, L., Liu, R., Zheng, L., Kumar, S., Vichare, P., and Dhokia, V. (2007) "Interoperable $\mathrm{CNC}$ for Global Manufacturing (Keynote paper)." Flexible Automation and Intelligent Manufacturing, FAIM2007, Philadelphia, USA, $1-13$. 
Nguyen, T., and Abdali, O. (2005). "Java Integrated Development Environment CASE Tool Comparison: Borland ${ }^{\circledR}$ JBuilder ${ }^{\mathrm{TM}}$ vs. NetBeans ${ }^{\circledR}$ IDE." School of Information Technology and Engineering (S.I.T.E.), Ottawa, Ontario, Canada.

Nielsen, J. (2003). "Information Modelling of Manufacturing Processes: Information Requirements for Process Planning in a Concurrent Engineering Environment," Doctoral Thesis, Royal Institute of Technology, Stockholm.

Nijssen, G. M., and Halpin, T. A. (1989). Conceptual Schema and Relational Database Design, A fact oriented approach, Prentice Hall, Upper Saddle River, NJ, USA.

Normile, D., Tyrka, K., Bulkeley, D., and Bak, D. (2000). "Design anywhere, build anywhere-Product Data Management and the Internet are redefining the relationships between manufacturers, customers, and suppliers." Global Design News.

OMAC. (2002). "The Value Proposition For STEP-NC, version 4." OMAC STEPNC WORKING GROUP. 26. http://www.omac.org/wgs/MachTool/STEPNC/Deliverables/STEP-NCValuePropDraftV.4.pdf.

Patil, L., and Pande, S. S. (2002). "An intelligent feature based process planning system for prismatic parts." International Journal of Production Research, $40(17), 4431-4447$.

Peng, T.-K., and Trappey, A. J. C. (1998). "Step toward STEP-compatible engineering data management: the data models of product structure and engineering changes." Robotics and Computer-Integrated Manufacturing, 14(2), 89-109. 
Proctor, F., Michaloski, J. L., and Shackleford, W. P. (2002) "Tying Together Design, Process Planning, and Machining with STEP-NC Technology." World automation congress; Robotics, manufacturing, automation and control trends, principles and applications, Orlando, FL, 33-38.

Quatrany, T. (1998). Visual Modelling with Rational Rose and UML, AddisonWesley, Reading, MA.

Rahimifard, S., and Newman, S. T. (1996). "A methodology to develop EXPRESS data models." International Journal of Computer Integrated Manufacturing, $9(1), 61-72$.

Rahimifard, S., and Newman, S. T. (1999). "The application of information systems for the design and operation of flexible machining cells." Journal of Intelligent Manufacturing, 10, 21-27.

Ravat, J., and Nazemetz, J. (2007). "Introduction to STEP." http://www.okstate.edu/indengr/step/WEBFILES/Papers/Introduction index.html.

Roger, N. N., Walt, B. W., and Philip, K. R. (1980). "Initial Graphics Exchange Specification IGES, version 1.0, NBSIR 80-1978." National Bureau of Standards, Washington (DC). http://www.nist.gov/sc4/national/usa/iges/iges.htm

Rosso-Jr, R. S. U. (2005). "STEP Compliant CAD/CAPP/CAM System For Rotational Asymmetric Parts," Doctoral Thesis, Loughborough, Loughborough, UK. 
Rosso-Jr, R. S. U., Newman, S. T., and Rahimifard, S. (2004). "The adoption of STEP-NC for the manufacture of asymmetric rotational components." Proceedings of the Institution of Mechanical Engineers Part B: J. Engineering Manufacture, 218, 1639-1644.

Schenck, D., and Wilson, P. (1994). Information Modelling the EXPRESS Way, Oxford University Press.

Shah, J. J., and Rogers, M. T. (1988). "Expert form feature modelling shell." Computer Aided Design, 20(9), 515-524.

Shah, J. J. R., M.T. (1998). "Functional requirements and conceptual design of the feature-based modelling system." Computer-Aided Engineering, 5(1), 9-15.

Shimamura, A., Moriyama, M., and Kasuga, K. (1996). "Data detection method for curve and surface data in STEP data exchange system." Seimitsu Kogaku Kaishi/Journal of the Japan Society for Precision Engineering, 62(5), 701 705.

Shin, S.-J., Suh, S.-H., and Stroud, I. (2007). "Reincarnation of G-code based part programs into STEP-NC for turning applications." Computer Aided Design, 39(1), 1-16.

Shunmugam, M. S., Mahesh, P., and Reddy, B. S. V. (2002). "A method of preliminary planning for rotational components with $\mathrm{C}$-axis features using genetic algorithm." Computers in Industry, 48, 199-217. 
Sokolov, A., Richard, J., Nguyen, V. K., Stroud, I., Maeder, W., and Xirouchakis, P. (2006). "Algorithms and an Extended STEP-NC Compliant Data Model for Wire Electro Discharge Machining Based on 3D Representations." International Journal of Computer Integrated Manufacturing, 19(6), 603613.

Srinivas, J. (2004). "Automatic generation of numerical control part programs using object-oriented software." Proceedings of the I Mech E Part B Journal of Engineering Manufacture, 218(2), 239-244).

STEP-NC-Project. (2000). "STEP-NC - New data interface for NC programming Issue 2, July 2000." STEP-NC, 4.

Storr, A., and Heusinger, S. (2002). "STEP-NC Basic of a CAD/NC process chain The STEP-NC process model for turning (Translation from Germany)."

Suh, S. H. (2002). "ISO 14649 Part 12 Process Data for Turning, WG3 T24presentation material." Seoul, Korea.

Suh, S. H., and Cheon, S. U. (2002). "A framework for an intelligent CNC and data model." International Journal of Advanced Manufacturing Technology, 19(10), 727-735.

Suh, S.-H., Cheon, S.-U., and Lee, B.-E. (2004). "Method for automatically generating part program for use in STEP-NC (US Patent 6795749)." http://www.patentstorm.us/patents/6795749-description.html.

Suh, S. H., Chung, D. H., Lee, B. E., Cho, J. H., Cheon, S. U., Hong, H. D., and Lee, H. S. (2002). "Developing an integrated STEP-compliant CNC prototype based on ISO 14649 Paradigm." Journal of Manufacturing Systems, 21(5), 350-362. 
Suh, S.-H., Chung, D.-H., Lee, B.-E., Shin, S., Choi, I., and Kim, K.-M. (2006). "STEP-compliant CNC system for turning: Data model, architecture, and implementation." Computer-Aided Design, 38(6), 677-688.

Suh, S. H., Lee, B. E., Chung, D. H., and Cheon, S. U. (2003). "Architecture and implementation of a shop-floor programming system for STEP-compliant CNC." Computer-Aided Design, 35(12), 1069-1083.

Tseng, Y. J., and Joshi, S. B. (1998). "Recognition of interacting rotational and prismatic machining features from 3-D mill-turn parts." International Journal of Production Research, 36(11), 3147-3165.

Usher, J. M., and Fernandes, K. J. (1999). "An object-oriented application dynamic process planning." International Journal of Production Research, 37(13), 2879-2894.

Vergeest, J. S. M. (1991). "CAD surface data exchange using STEP." Computer Aided Design, 23(4), 269-281.

Wang, H., and Xu, X. (2004) "A STEP-Compliant "Adaptor" for Linking CAPP with CNC." The 34th International Matador Conference, Manchester, UK, $45-50$.

Wang, H.-F., and Zhang, Y.-L. (2002). "CAD/CAM integrated system in collaborative development environment." Robotic and Computer Integrated Manufacturing, 18(2), 135-145.

Williams, D. J. (1988). Manufacturing Systems: an introduction to technologies, Stony Stratford: Open University Press. 
Wilson, P. R. (1998) "STEP and EXPRESS.". NSF Workshop on Distributed Information, Computation and Process Management for Scientific and Engineering Environments, Virginia, USA.

$\mathrm{Xu}, \mathrm{X}$., and Newman, S. T. (2006). "Making CNC Machine Tools More Open, Interoperable and Intelligent." Computers in Industry, 57(2), 141-152.

Xu, X., and Wang, J. (2004) "Development of a G-Code Free, STEP-Compliant CNC Lathe." Proc of the 2004 International Mechanical Engineering Congress and Exposition (IMECE), Anaheim, California, U.S.A.

$\mathrm{Xu}, \mathrm{X}$. W. (2006). "Realization of STEP-NC enabled machining." Robotics and Computer-Integrated Manufacturing, 22, 144-153.

$\mathrm{Xu}, \mathrm{X}$. W., and He, Q. (2004). "Striving for a total integration of CAD, CAPP, CAM and CNC." Robotics and Computer-Integrated Manufacturing, 20(2), 101-109.

Xu, X. W., Klemm, P., Proctor, F. M., and Suh, S. H. (2006a). "STEP-Compliant Process Planning and Manufacturing." International Journal of Computer Integrated Manufacturing, 19(6), 491-494.

Xu, X. W., Wang, H., Mao, J., Newman, S. T., Kramer, T. R., Proctor, F. M., and Michaloski, J. L. (2005). "STEP-Compliant NC Research: The search for Intelligent CAD/CAPP/CAM/CNC Integration.". International Journal of Production Research, 43(17), 3703-3743.

Xu, X. W., Wang, L., and Rong, Y. (2006b). "STEP-NC and function blocks for interoperable manufacturing." IEEE Transactions on Automation Science and Engineering, 3(July), 297-308. 
Yamada, H., Tanaka, F., and Onosato, M. (2006) "Analysis of ISO 6983 NC Data Based on ISO 14649 CNC Data Model." International Federation for Information Processing (IFIP), Knowledge Enterprise: Intelligent Strategies in Product Design, Manufacturing, and Management, Shanghai, China, 109-114.

Yang, H., Lu, W. F., and Lin, A. C. (1994). "PROCASE: a case-based process planning system for machining of rotational parts." Journal of Intelligent Manufacturing, 5, 411-430.

Yildiz, Y., Korkut, Y., and Peker, O. U. (2006). "Development of a Feature Based CAM System for Rotational Parts." G.U Journal of Science, 19(1), 35-40.

Young, R. I. M. (2003). "Informing decision-makers in product design and manufacture." International Journal of Computer Integrated Manufacturing, $16(6), 428-438$.

Younis, M. A., and Wahab, A. M. A. (1997). "A CAPP Expert System for rotational components." Computers and Industrial Engineering, 33(3-4), 509-512.

Yusof, Y., Case, K., Newman, S. T., and Xu., X. W. (2007) "A STEP Compliant System for Turning Operations." 17th International Conference on Flexible Automation and Intelligent Manufacturing (2007 FAIM), Philadelphia, USA, 140-147.

Yusof, Y., Newman, S. T., and Case, K. (2006a) "Machining of rotational asymmetric components with a single turning centre." Proceedings of the 8th Biennial ASME Conference on Engineering Systems Design and Analysis, ESDA2006, Torino, Italy, 1-9. 
Yusof, Y., Rosso-Jr, R. S. U., Newman, S. T., and Case, K. (2006b) "The Design of a STEP-NC Compliant CAD/CAPP/CAM System for the Manufacture of Rotational Parts on a CNC Turning Centre." Proceedings of the 23rd International Manufacturing Conference (IMC23), University of Ulster, Northern Ireland, UK, 19-28.

Zeid, I. (1991). CAD/CAM Theory and Practice, McGraw Hill Inc., New York.

Zhang, C., Liu, R., and Hu, T. (2006). "On the Futuristic Machine Control in a STEP-Compliant Manufacturing Scenario." International Journal of Computer Integrated Manufacturing, 19(6), 508-515.

Zhou, X., Qiu, Y., Hua, G., Wang, H., and Ruan, X. (2007). "A feasible approach to the integration of CAD and CAPP." Computer-Aided Design, 39(4), 324338. 


\section{Appendix A - Author Publications}

1. Yusof, Y., Newman, S.T. and Case, K., "STEP Compliant NC for Interoperable Manufacture of Turn/Mill Discrete Components", Challenges Facing Manufacturing, J. Vickery (ed), Institute of Technology Tallaght, Proceedings of the 22nd International Manufacturing Conference (IMC22), Institute of Technology, Tallaght, Dublin, Ireland, August 2005, pp 529-536, ISBN 0-9551218-0-9.

2. Yusof, Y., Newman, S.T., Case, K. and Rosso Jr., R.S.U., "Interoperable Process Planning Facility for Turn/Mill Components", The International Advanced Technology Congress (ATCi2005), Proceedings of the International Conference on Computer Integrated Systems 2005 (ConCIS 2005), Selangor, Malaysia, December 2005, pp 33-40.

3. Yusof, Y., Case, K. and Newman, S.T., "Machining of rotational asymmetric components with a single turning centre", Proceedings of the 8th Biennial ASME Conference on Engineering Systems Design and Analysis, ESDA2006, Torino, Italy, 4th July 2006, p.p.1-9, ISBN:0-7918-3779-3, [Also on CD-ROM].

4. Yusof, Y., Rosso-Jr, R. S. U., Case, K. and Newman, S. T., "The design of a STEP-NC compliant CAD/CAP/CAM system for the manufacture of rotational parts on a CNC turning centre, in 'Innovations in Manufacturing'", Proceedings of the 23rd International Manufacturing Conference, University of Ulster, 30th August - 1st September 2006, University of Ulster, September 2006, p.p.19-28, [CD-ROM]. 
5. Y. Yusof, K. Case, S. T. Newman and X. W. Xu. "A STEP Compliant System for Turning Operations". Proceedings of the 17th International Conference on Flexible Automation and Intelligent Manufacturing (2007 FAIM), June 18-20, 2007, Penn State Great Valley, Philadelphia, USA. pp 140-147, ISBN: 978-1-4276-2092-7.

6. Yusof, Y., Case, K., and Newman, S.T. " Application of STEP-NC for CNC Machining of Asymmetrical Components " , Proceedings of the 5th International Conference on Manufacturing Research (ICMR '07), De Montfort University, Leicester, UK, September 11-13, 2007, pp 89-93, ISBN 978-0-9556714. 


\section{Appendix B - Summaries of Standards Document}

\section{Standards by Name and Number}

1. Selected STEP Standards - Mechanical

i. AP203 - Configuration controlled design of mechanical parts and assemblies (ISO 10303-203:1994)

ii. AP213 - Numerical control process plans for machined parts (ISO/DIS 10303-213) - cancelled and replaced by AP240 (below)

iii. AP214 - Core data for automotive mechanical design processes (ISO 10303-214:2001)

iv. AP224 - Mechanical product definition for process planning using machining features (ISO 10303-224:1999 (Edition 1) \& ISO 10303224:2001 (Edition 2)

v. AP238 - Application interpreted model for numerical controllers (ISO/CD 10303-238) (CD Ballot successful in Fourth Quarter 2002)

vi. AP240 - Process plans for machined parts (ISO/CD 10303-340) (DIS Ballot during First Quarter 2004)

2. Related ISO Standards

i. ISO 14649 (ISO TC184/SC1/WG7) --- Physical device control - Data model for computerized numerical controllers

a. Part 1 - Overview and fundamental principles (ISO/FDIS 14649-1)

b. Part 10 - General process data (ISO/FDIS 14649-10)

c. Part 11 - Process data for milling (ISO/FDIS 14649-11)

d. Part 12 - Process data for turning (ISO/CD 14649-12)

e. Part 111 - Tools for milling (ISO/FDIS 14649-111)

f. Part 121 - Tools for turning (ISO/CD 14649-121)

ii. ISO 13399 (ISO TC29/WG34) --- Cutting tool representation and exchange

iii. ISO 6983-1:1982 (TC184/SC1) - Numerical control of machines Program format and definition of address words - data format for positioning, line motion, and contouring control systems 


\section{Summaries of Standard Documents}

\begin{tabular}{|c|c|}
\hline Documents & Scope \\
\hline AP203 & $\begin{array}{l}\text { - Products that are mechanical parts and assemblies; } \\
\text { - Product definition data and configuration control data pertaining } \\
\text { to the design phase of a product's development; } \\
\text { - The change of a design and data related to the documentation of } \\
\text { the change process; } \\
\text { - Five types of shape representations of a part, including } \\
\text { wireframe and surface without topology, wireframe geometry } \\
\text { with topology, manifold surfaces with topology, faceted } \\
\text { boundary representation, and boundary representation; } \\
\text { - Alternate representations of the data by different disciplines } \\
\text { during the design phase of a product's life cycle; } \\
\text { - Identification of government, industry, company or other } \\
\text { specifications for design, process, surface finish, and materials } \\
\text { which are specified by a designer as being applicable to the } \\
\text { design of the product; } \\
\text { - Identification of government, industry, company, or other } \\
\text { standard parts for their inclusion in a product's design; } \\
\text { - Data that are necessary for the tracking of a design's release; } \\
\text { - Data that are necessary to track the approval of a design, a } \\
\text { design aspect, or a configuration control aspect of a product; } \\
\text { - - If a part is being designed under a contract, the identification } \\
\text { of, and reference to, that contract under which the design is } \\
\text { developed; } \\
\text { - The identification of the security classification of a single part } \\
\text { when it is a component in an assembly; } \\
\text { - Data that is used in, or results from, the analysis or test of a } \\
\text { design that is used as evidence when considering a change to a } \\
\text { design }\end{array}$ \\
\hline $\begin{array}{c}\text { AP213 } \\
\text { (Replaced by } \\
\text { AP240) } \\
\text { (See Below) }\end{array}$ & $\begin{array}{l}\text { - Information from the planning activity that is contained in the } \\
\text { NC process plans for machined parts; } \\
\text { - Work instructions for the tasks required to manufacture a part, } \\
\text { using numerical control. These instructions include: } \\
\text { - References to the resources required to perform the work } \\
\text { - The sequences of the work instructions } \\
\text { - Relationships of the work to the part geometry } \\
\text { - References to standards and specifications declared in the } \\
\text { process plan; } \\
\text { - Information required to support the NC programming of } \\
\text { processes specified in the process plan; } \\
\text { - Information required to support in-process inspection specified } \\
\text { in the process plan; } \\
\text { Note - In-process inspection includes such tasks as using gage } \\
\text { blocks or performing a probing operation to verify the }\end{array}$ \\
\hline
\end{tabular}




\begin{tabular}{|c|c|}
\hline & $\begin{array}{l}\text { dimensional constraints placed upon the part. } \\
-\quad \text { Shop floor information specified in the process plan. }\end{array}$ \\
\hline \multirow[t]{2}{*}{ AP214 } & $\begin{array}{l}\text { Products of automotive manufacturers and their suppliers. These } \\
\text { products include parts, assemblies of parts, tools, assemblies of } \\
\text { tools, and raw materials. The considered parts include the } \\
\text { constituents of the car body, of the power train, of the chassis, } \\
\text { and of the interior. The tools include those specific to the } \\
\text { products produced and used by various manufacturing } \\
\text { technologies. Example: Typical manufacturing technologies } \\
\text { are for: } \\
\text { - Primary shaping: moulding or casting } \\
\text { - Transforming: bending or stamping } \\
\text { - Separating: milling or turning } \\
\text { - Coating: painting or surface coating } \\
\text { - Fitting: welding or riveting }\end{array}$ \\
\hline & $\begin{array}{l}\text { - Raw materials used to produce the parts or the tools; } \\
\text { - Process plan information describing the relationships between } \\
\text { parts, the tools used to manufacture them, and the tools used to } \\
\text { manage the relationships between intermediate stages of part or } \\
\text { tool development; }\end{array}$ \\
\hline AP214 & $\begin{array}{l}\text { - Product definition data and configuration control data for } \\
\text { managing large numbers of automotive products during the } \\
\text { design phase; } \\
\text { - Data describing the changes that have occurred during the } \\
\text { design phase, including tracking of the versions of a product } \\
\text { and of the data related to the change-process documentation; } \\
\text { - Identification of physically realized parts or tools (e.g., for } \\
\text { prototype building); } \\
\text { - Identification of standard parts, based on international, national, } \\
\text { or industrial standards; } \\
\text { - Release and approval data for product data; } \\
\text { - Data that identify the supplier of a product and related contract } \\
\text { information; } \\
\text { - The following eight types of representation of the shape of a part } \\
\text { or tool: } \\
\text { - 2D - wireframe representation } \\
\text { - 3D - wireframe representation } \\
\text { - Geometrically bounded surface representation } \\
\text { - Topologically bounded surface representation } \\
\text { - Faceted - boundary representation } \\
\text { - Boundary representation } \\
\text { - Compound shape representation } \\
\text { - Constructive solid geometric representation } \\
\text { - Representation of the shape of parts or tools that is a } \\
\text { combination of two or more of these eight types of shape } \\
\text { representation; }\end{array}$ \\
\hline
\end{tabular}




\begin{tabular}{|c|c|}
\hline & $\begin{array}{l}\text { - Data that pertains to the presentation of the shape of the product; } \\
\text { - Representation of portions of the shape of a part or tool by form } \\
\text { features; } \\
\text { - Product documentation represented on drawings; } \\
\text { - References to product documentation represented in a format } \\
\text { other than those specified by ISO 10303; } \\
\text { Example: Other formats include physical clay models, digital data } \\
\text { in other standard formats such as NC - data encoded according to } \\
\text { ISO 6983, or text data encoded according to ISO/IEC } 8879 \\
\text { (SGML) }\end{array}$ \\
\hline AP214 & $\begin{array}{l}\text { Simulation data for the description of kinematics structures; } \\
\text { Example: The simulation data for a windshield wiper may } \\
\text { include the geometry of the windshield as well as the } \\
\text { kinematics structure of the wiper, including all necessary links } \\
\text { and joints. } \\
\text { - Properties of parts or tools; } \\
\text { - Data defining surface conditions; } \\
\text { - Dimensional data and geometrical tolerance data. }\end{array}$ \\
\hline AP238 & $\begin{array}{l}\text { - This part of ISO } 10303 \text { specifies the application interpreted } \\
\text { model for the information requirements defined by the ISO } 14649 \\
\text { Data model for computerized numerical controllers. } \\
\text { - This part of ISO } 10303 \text { also specifies the use of the integrated } \\
\text { resources necessary for the scope and information requirements of } \\
\text { numerical controlled machining and associated processes, } \\
\text { including the scope and information requirements defined by ISO } \\
\text { 14649. } \\
\text { Note - The application activity model in the overview and the } \\
\text { fundamental principles in ISO 14649-1 describe the processes and } \\
\text { information flows that are the basis for the definition of the scope } \\
\text { of this part of ISO } 10303 \text {. } \\
\text { - The following are within the scope of this part of ISO 10303: } \\
\text { - Mechanical parts for manufacturing; } \\
\text { - Manufacturing process descriptions, including } \\
\text { manufacturing operations, sequences of operations, and } \\
\text { associated information as defined in ISO 14649; } \\
\text { - The AS-IS and TO-BE shapes of a mechanical part; } \\
\text { - Manufacturing features of a part; } \\
\text { - Manufacturing tolerance requirements of a part; } \\
\text { - Tool requirements for machining operations; } \\
\text { - Tool paths for machining operations; } \\
\text { - Manufacture of mechanical products, using } \\
\text { manufacturing processes defined in ISO 14649; } \\
\text { - Manufacturing product discipline view. }\end{array}$ \\
\hline & $\begin{array}{l}\text {-This part of ISO } 10303 \text { specifies the use of the integrated } \\
\text { resources necessary to define the scope and information }\end{array}$ \\
\hline
\end{tabular}




\begin{tabular}{|c|c|}
\hline & $\begin{array}{l}\text { requirements of the exchange, sharing, and long-term data } \\
\text { retention of computer-interpretable process plan information for } \\
\text { both numerical control (NC) and manually operated applications } \\
\text { and associated product definition data. } \\
\text { Note - The application activity model provides a graphical } \\
\text { representation of the processes and information flows, which are } \\
\text { the basis for defining the scope of this part of ISO 10303. } \\
\text { - The following are within the scope of this part of ISO 10303: } \\
\text { - Information out of the planning activity that is contained } \\
\text { in the process plans for machined parts, including: } \\
\text { Numerical controlled machines Manual operations }\end{array}$ \\
\hline AP240 & $\begin{array}{l}\text { The manufacture of a single-piece mechanical part and } \\
\text { assemblies of single-piece parts for manufacturing } \\
\text { purposes, including: } \\
\text { i. Process data for part routing, which includes } \\
\text { manufacturing process and setup sequencing } \\
\text { ii. Process data for operation } \\
\text { - Interface for capturing technical data out of the upstream } \\
\text { application protocols, including: } \\
\text { i. Product definition data, which includes tracking a } \\
\text { design exception notice of a part } \\
\text { ii. Initial material definition data } \\
\text { - Technical data for and out of the process planning for } \\
\text { machined parts, including: } \\
\text { i. Machining features for defining the shapes } \\
\text { necessary for manufacturing } \\
\text { ii. Machining feature classification structure } \\
\text { iii. Geometric and dimensional tolerances of the parts } \\
\text { iv. Materials and properties of the parts being } \\
\text { manufactured } \\
\text { - References to standards and specifications declared in } \\
\text { the process plan; } \\
\text { - Work instructions for the tasks required to manufacture } \\
\text { a part, using numerical control, including: } \\
\text { i. References to the resources required to perform the } \\
\text { ii. The sequences of the work instructions } \\
\text { iii. Relationships of the work to the part geometry } \\
\text { - Information required to support NC programming of } \\
\text { processes specified in the process plan; } \\
\text { - Information required to support the in-process } \\
\text { gauge blocks and performing a probing operation to } \\
\text { verify the dimensional constraints placed upon the part. }\end{array}$ \\
\hline
\end{tabular}




\begin{tabular}{|c|c|}
\hline & $\begin{array}{l}\text { - Shop floor information specified in the process plan. } \\
\text { Note - Shop floor information contains items such as } \\
\text { part routing, machine setup, and part loading } \\
\text { instructions. }\end{array}$ \\
\hline ISO14649-1 & $\begin{array}{l}\text { Includes a data model for computerized numerical controllers, } \\
\text { including an overview and fundamental principles. }\end{array}$ \\
\hline ISO14649-10 & $\begin{array}{l}\text {-This part of ISO } 14649 \text { specifies the process data that is } \\
\text { generally needed for NC programming within all machining } \\
\text { technologies. These data elements describe the interface between } \\
\text { a computerized numerical controller and the programming system } \\
\text { (i.e., CAM system or shopfloor programming system). On the } \\
\text { programming system, the program for the numerical controller is } \\
\text { created. This program includes geometric and technological } \\
\text { information. It can be described using this part of ISO } 14649 \\
\text { together with the technology-specific parts (such as ISO } 14649 \text { - } \\
\text { 11). This part of ISO } 14649 \text { provides the control structures for the } \\
\text { sequence of program execution, which is mainly the sequence of } \\
\text { working steps and associated machine functions. } \\
\text {-The "machining schema" defined in this part of ISO } 14649 \\
\text { contains the definition of data types that are generally relevant for } \\
\text { different technologies (e.g., milling, turning, grinding). The } \\
\text { features for non-milling technologies like turning and EDM will } \\
\text { be introduced when the technology-specific parts (such as ISO } \\
\text { 14649-12 for turning, ISO 14649-13 for EDM, and ISO 14649-14 } \\
\text { for contour cutting of wood and glass) are published. }\end{array}$ \\
\hline ISO14649-10 & $\begin{array}{l}\text { This schema includes the definition of the workpiece, a feature } \\
\text { catalogue containing features that might be referenced by several } \\
\text { technologies, the general executables, and the basis for an } \\
\text { operation definition. } \\
\text {-This part of ISO } 14649 \text { cannot stand alone. An implementation } \\
\text { needs at least one technology-specific part (e.g., ISO 14649-11 } \\
\text { for milling or ISO } 14649-12 \text { for turning). } \\
\text {-Additionally, the schema uses machining features similar to ISO } \\
\text { 10303-224 and ISO 10303-214. The description of process data is } \\
\text { done using the EXPRESS language defined in ISO } 1030311 \text {. The } \\
\text { encoding of the data is done using ISO 10303-21. }\end{array}$ \\
\hline ISO14649-11 & $\begin{array}{l}\text { - This part of ISO14649 specifies the data elements needed as } \\
\text { process data for milling. This part of ISO } 14649 \text { also specifies the } \\
\text { technology-specific data elements needed as process data for } \\
\text { milling. } \\
\text {-Together with the general process data described in ISO } 14649 \text { - } \\
10 \text {, this part of ISO } 4649 \text { describes the interface between a } \\
\text { computerized numerical controller and the programming system }\end{array}$ \\
\hline
\end{tabular}




\begin{tabular}{|c|c|}
\hline ISO14649-11 & $\begin{array}{l}\text { (i.e., CAM system or shopfloor programming system) used for } \\
\text { milling. It can be used for milling operations on all types of } \\
\text { machines, such as milling machines, machining centres, or lathes } \\
\text { with motorized tools capable of milling. } \\
\text {-The subject of the milling schema, which is described in this } \\
\text { part of ISO } 14649 \text {, is the definition of technology-specific data } \\
\text { types representing the machining process for milling and drilling. } \\
\text { This includes both milling of freeform surfaces as well as milling } \\
\text { of prismatic workpieces (also known as } 21 / 2 \text { D-milling). Not } \\
\text { included in this schema are geometric items, representations, } \\
\text { manufacturing features, executable objects, and base classes that } \\
\text { are common for all technologies. These are referenced from ISO } \\
\text { 10303's generic resources and from ISO 14649-10. Process data } \\
\text { is described using the EXPRESS language defined in ISO 10303- } \\
11 \text {. The encoding of the data is done using ISO 10303-21. }\end{array}$ \\
\hline $\begin{array}{c}\text { ISO } 14649 \\
12\end{array}$ & $\begin{array}{l}\text {-This part of ISO14649 specifies the data elements needed as } \\
\text { process data for turning. This part of ISO } 14649 \text { also specifies the } \\
\text { technology-specific data elements needed as process data for } \\
\text { turning. } \\
\text {-Together with the general process data described in ISO 14649- } \\
10 \text {, it describes the interface between a computerized numerical } \\
\text { controller and the programming system (i.e., CAM system or } \\
\text { shopfloor programming system) used for turning. It can be used } \\
\text { for turning operations on all types of machines }\end{array}$ \\
\hline $\begin{array}{c}\text { ISO14649- } \\
111\end{array}$ & $\begin{array}{l}\text {-This part of ISO } 14649 \text { specifies the data elements needed as } \\
\text { tools for milling. They work together with ISO 14649-11, which } \\
\text { includes the process data for milling. These data elements can be } \\
\text { used as the criteria for selecting one of several milling and drilling } \\
\text { type tools, not to describe the complete information about a } \\
\text { particular tool. Therefore, leaving out optional attributes gives the } \\
\text { controller more freedom to select from a larger set of tools. The } \\
\text { NC is assumed to have access to the complete description of } \\
\text { specific tools in a database. } \\
\text {-The milling tool_schema defined in this part of ISO 14649 } \\
\text { serves as a basic tool schema that includes only the most } \\
\text { important information. It is intended to give the controller enough } \\
\text { information to select the tool specified in the NC program. } \\
\text {-In ISO 6983, the tool is defined just with its number (e.g., T8). } \\
\text { No further information concerning the tool type or geometry is } \\
\text { given. This information is part of the tool set-up sheet, which is } \\
\text { supplied with the machine's NC program. The tool set-up sheet } \\
\text { gives the relationship between the tool location (e.g., slot } 8 \text { ) and } \\
\text { the type of tool (e.g., "drill } 4 \text { mm"). }\end{array}$ \\
\hline
\end{tabular}




\begin{tabular}{|c|c|}
\hline & $\begin{array}{l}\text {-The approach of this tool set-up sheet to ISO } 14649-11 \text { is to } \\
\text { include the information that is contained in the NC program's tool } \\
\text { set-up sheet mentioned above. Therefore, the most important } \\
\text { information that needs to be included in the tool description is: }\end{array}$ \\
\hline $\begin{array}{c}\text { ISO14649- } \\
111\end{array}$ & $\begin{array}{l}\text { - Tool type } \\
\text { - Tool geometry } \\
\text { - Expected tool life } \\
\text {-The tool schema does not include information that is part of the } \\
\text { tool database. The tool database is related to the machine and the } \\
\text { tools themselves but is independent of the NC program. This } \\
\text { means that data like the following data types are not included in } \\
\text { the tool schema: } \\
\text { - Normative tool life } \\
\text { - Tool location in the tool changer } \\
\text {-It is important to understand that all length measure types used in } \\
\text { this Part are not tolerances length measure types, because they are } \\
\text { used to describe the tools required for the manufacturing of a } \\
\text { workpiece, not the actual dimensions of the tools available at the } \\
\text { machine. Tool management must select a real tool based on the } \\
\text { actual tool dimensions and the tolerances of features. } \\
\text {-The overall structure of the tool description in this part of ISO } \\
\text { 14649 and ISO 14649-10 is the same one in ISO/DIS 13399-1. } \\
\text { Many definitions of tool body and its geometry are referenced } \\
\text { from the NIST tool model [NISTIR5707:Modeling of } \\
\text { Manufacturing Resource Information, July,1995. }\end{array}$ \\
\hline $\begin{array}{c}\text { ISO14649- } \\
121\end{array}$ & $\begin{array}{l}\text {-This part of ISO } 14649 \text { specifies the data elements needed as } \\
\text { tools for turning. They work together with ISO } 14649-12 \text {, which } \\
\text { includes the process data for turning. These data elements can be } \\
\text { used as a criteria to select one of several turning type tools, not to } \\
\text { describe the complete information about a particular tool. } \\
\text { Therefore, leaving out optional attributes gives the controller } \\
\text { more freedom to select from a larger set of tools. The NC is } \\
\text { assumed to have access to the complete description of specific } \\
\text { tools in a database. } \\
\text {-The turning tool schema defined in this part of ISO } 14649 \\
\text { serves as a basic tool schema that includes only the most } \\
\text { important information. It is intended to give the controller enough } \\
\text { information to select the tool specified in the NC program. }\end{array}$ \\
\hline
\end{tabular}




\section{Appendix C - Turning Features Library Codes}

The following code is taken from general class "turning features" library (TFL). In the code, Parent machining features and member machining features are declared as public variables respectively, to be able to call them from anywhere in the feature modeller. This will also make each parent feature a subclass of turning features class. This code also presents how the inheritance structure is constructed throughout the TFL file.

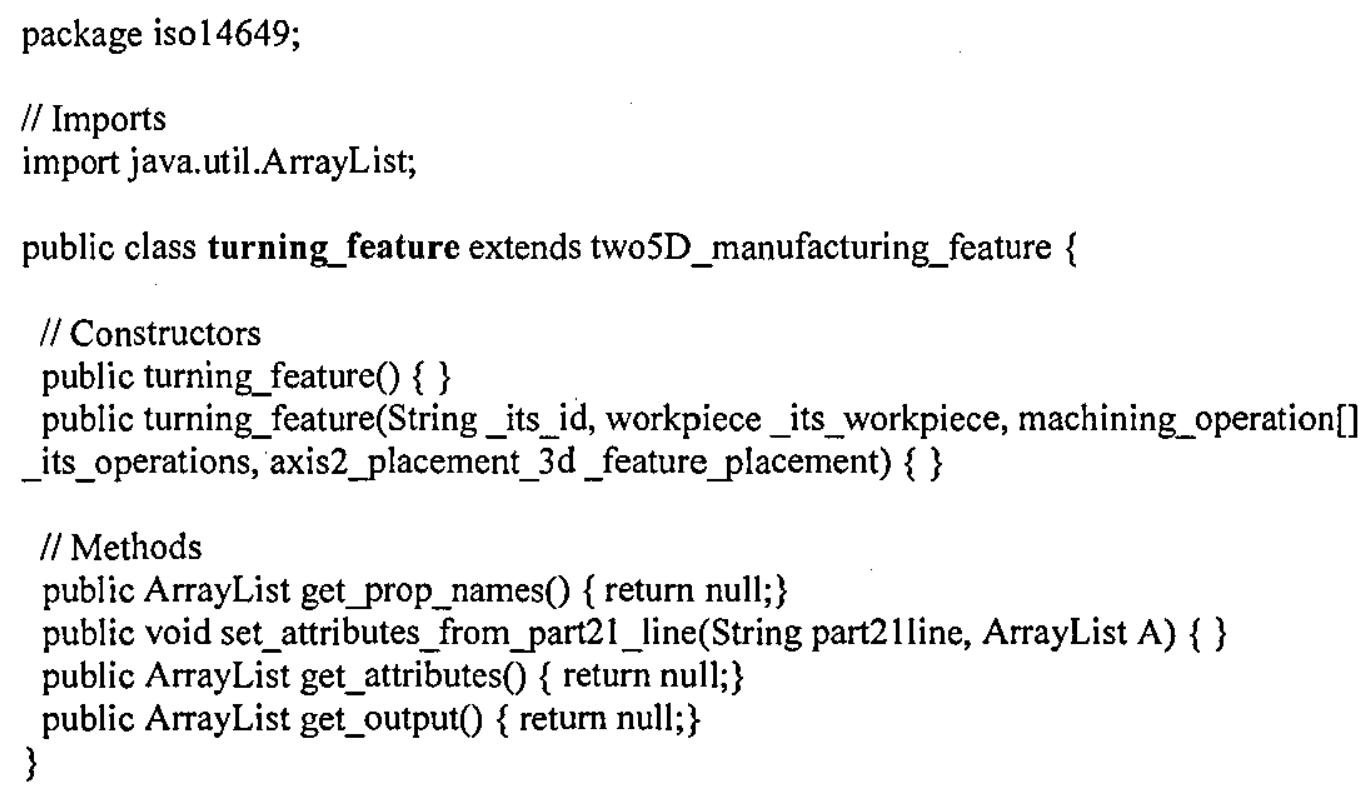


package iso 14649;

// Imports

import java.util.ArrayList;

public class outer_diameter extends outer_round \{

// Fields

public toleranced_length_measure diameter_at_placement;

public toleranced_length_measure feature_length;

public Object reduced_size;

// Constructors

public outer_diameter() \{\}

public outer_diameter(String_its_id, workpiece _its_workpiece, machining_operation[]

_its_operations, axis2_placement_3 3 d_feature_placement, toleranced_length_measure

_diameter_at_placement, toleranced_length_measure_feature_length, Object

_reduced_size) \{\}

// Methods

public ArrayList get_prop_names() \{ return null;

public void set_attributes_from_part21_line(String part21line, ArrayList A) \{\}

public void set_diameter_at_placement(toleranced_length_measure

_diameter_at_placement) \{\}

public void set_feature_length(toleranced_length_measure_feature_length) \{\}

public void set_reduced_size(Object_reduced_size) \{\}

public toleranced_length_measure get_diameter_at_placement() \{return null; $\}$

public toleranced_length_measure get_feature_length ()$\{$ return null; $\}$

public Object get_reduced_size() \{return null; $\}$

public ArrayList get_attributes() \{return null; \}

\}

public ArrayList get_output() \{return null; \}

package iso 14649;

// Imports

import java.util.ArrayList;

public class outer_diameter_to_shoulder extends outer_round \{

// Fields

public toleranced_length_measure diameter_at_placement;

public vee_profile v_shape_boundary;

// Constructors

public outer_diameter_to_shoulder() \{\} 
public outer_diameter_to_shoulder(String_its_id, workpiece_its_workpiece, machining_operation[]_its_operations, axis 2 _placement_3d_feature_placement, toleranced_length_measure _diameter_at_placement, vee_profile_v_shape_boundary) \{\}

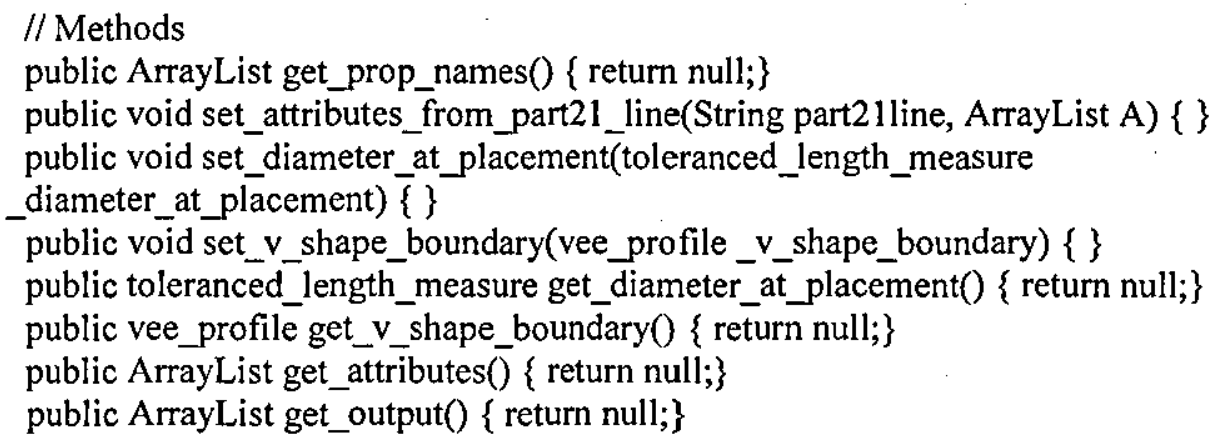




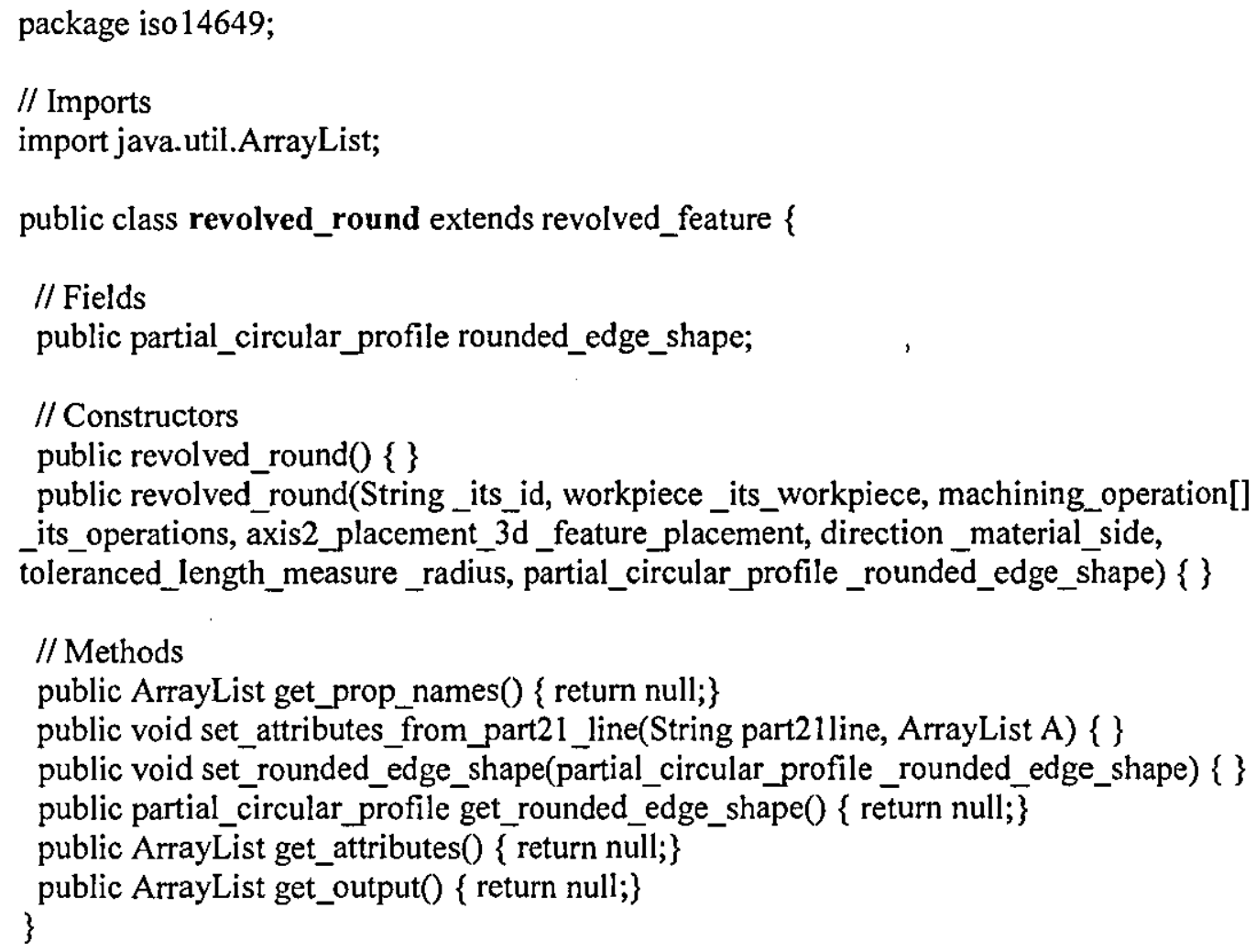


package iso14649;

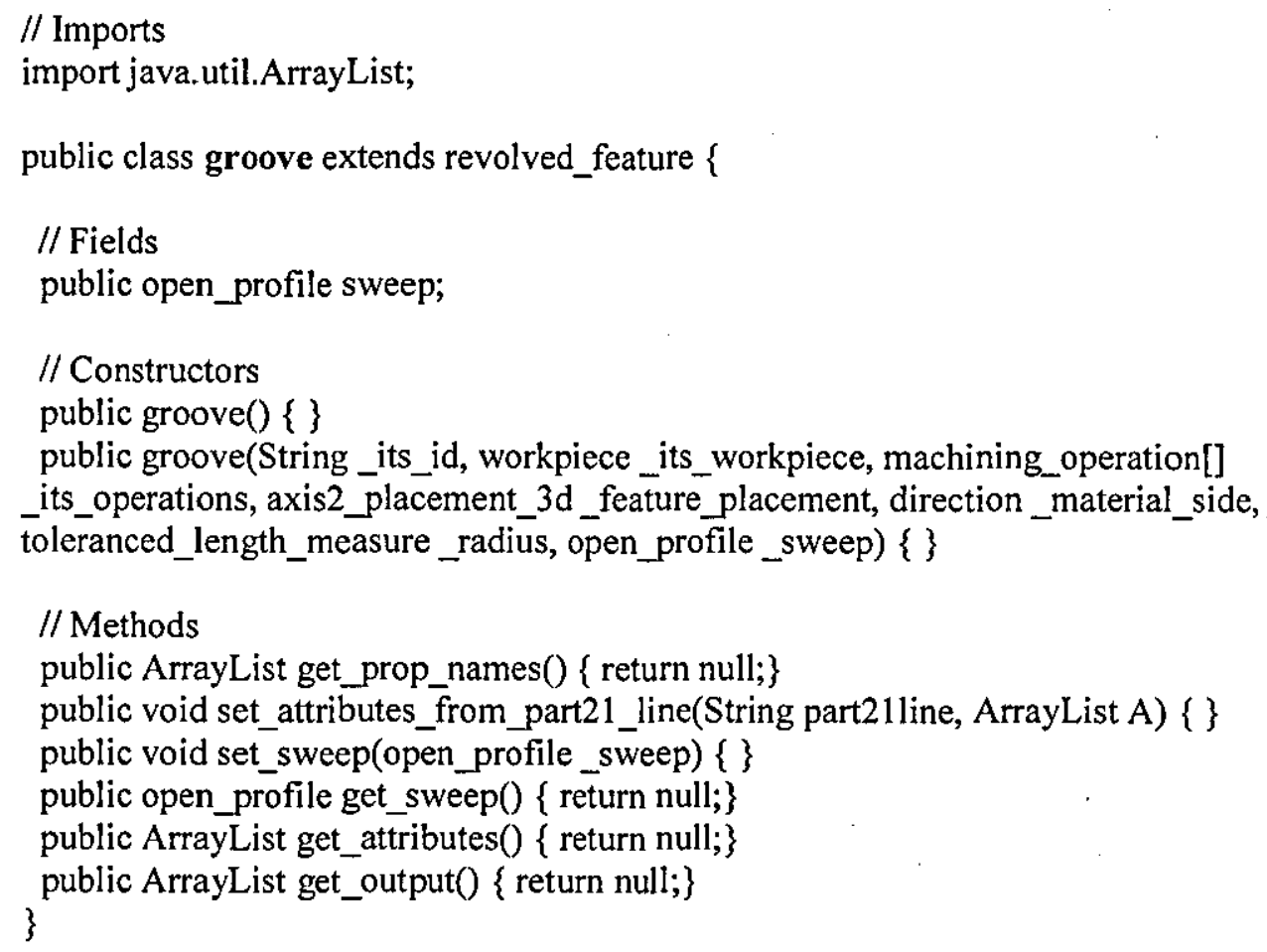


package isol4649;

// Imports

import java.util.ArrayList;

public class knurl extends turning_feature \{

// Fields

public turning_feature base_feature;

public partial_area_definition partial_profile;

public toleranced_length_measure tooth_depth;

public toleranced_length_measure diametral_pitch;

public toleranced_length_measure root_fillet;

public Integer number_of teeth;

public toleranced_length_measure major_diameter;

public toleranced_length_measure nominal_diameter;

// Constructors

public knurl() \{\}

public knurl(String _its_id, workpiece_its_workpiece, machining_operation[]

_its_operations, axis 2 _placement_3d_feature_placement, turning_feature _base_feature,

partial_area_definition_partial_profile, toleranced_length_measure _tooth_depth,

toleranced_length_measure _diametral_pitch, toleranced_length_measure_root_fillet,

Integer_nümber_of_teeth, toleranced_length_measure_major_diameter,

toleranced_length_measure_nominal_diameter) \{\}

\section{// Methods}

public ArrayList get_prop_names() \{ return null;

public void set_attributes_from_part21_line(String part21line, ArrayList A) \{\}

public void set_base_feature(turning_feature base_feature) \{\}

public void set_partial_profile(partial_area_definition_partial_profile) \{\}

public void set_tooth_depth(toleranced_length_measure_tooth_depth) \{\}

public void set_diametral_pitch(toleranced_length_measure_diametral_pitch) \{\}

public void set_root_fillet(toleranced_length_measure _root_fillet) \{\}

public void set_number_of_teeth(Integer_number_of_teeth) \{\}

public void set_major_diameter(toleranced_length_measure_major_diameter) \{\}

public void set_nominal_diameter(toleranced_length_measure_nominal_diameter) \{\}

public turning_feature get_base_feature() \{return nulli;

public partial_area_definition get_partial_profile() \{return null; \}

public toleranced_length_measure get_tooth_depth() \{return null; $\}$

public toleranced_length_measure get_diametral_pitch() \{return null; $\}$

public toleranced_length_measure get_root_fillet() \{return null; $\}$

public Integer get_number_of_teeth() \{return null; $\}$

public toleranced_length_measure get_major_diameter() \{return null; $\}$

public toleranced_length_measure get_nominal_diameter() \{return null; $\}$

public ArrayList get_attributes() \{ return null; \}

public ArrayList get_output() \{return null; $\}$ 
package iso14649;

// Imports

import java.util.ArrayList;

public class straight_knurl extends knurl \{

\section{// Constructors}

public straight_knurl() \{ \}

public straight_knurl(String_its_id, workpiece_its_workpiece, machining_operation[] its_operations, axis2 placement_3d feature placement, turning_feature _base_feature, partial_area_definition_partial_profile, toleranced_length_measure _tooth_depth, toleranced_length_measure _diametral_pitch, toleranced_length_measure _root_fillet, Integer_number_of_teeth, toleranced_length_measure_major_diameter, toleranced_length_measure_nominal_diameter) \{\}

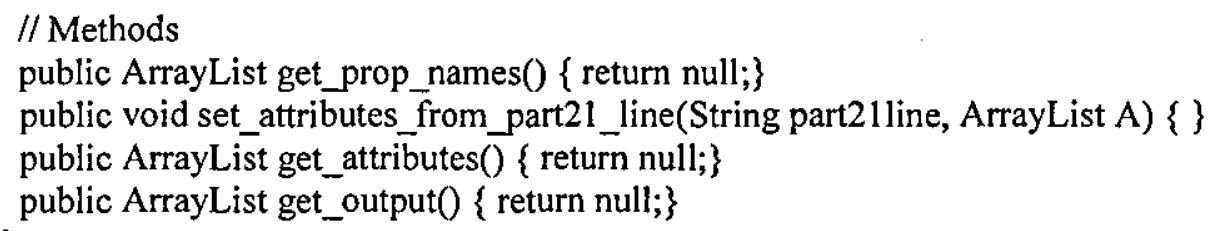


package iso14649;

// Imports

import java.util.ArrayList;

public class diamond_knurl extends knurl \{

//Fields

public Double helix1_angle;

public Double helix2_angle;

// Constructors

public diamond_knurl() \{\}

public diamond_knurl(String_its_id, workpiece_its_workpiece, machining_operation[] _its_operations, axis__placement_3 $3 \mathrm{~d}$ _feature_placement, turning_feature _base_feature, partial_area_definition_partial_profile, toleranced_length_measure _tooth_depth, toleranced_length_measure _diametral_pitch, toleranced_length_measure _root_fillet, Integer_number_of_teeth, toleranced_length_measure_major_diameter, toleranced_length_measure_nominal_diameter, Double_helix 1 _angle, Double _helix2_angle) $\{\overline{\}}$

\section{// Methods}

public ArrayList get_prop_names() \{return null; $\}$

public void set_attributes_from_part21_line(String part21line, ArrayList A) \{\}

public void set_helixl_angle(Double_helix1_angle) \{\}

public void set_helix2_angle(Double_helix2_angle) \{\}

public Double get helixl_angle() \{return null; $\}$

public Double get_helix2_angle() \{return null; $\}$

public ArrayList get_attributes() \{return null; $\}$

\}

public ArrayList get_output() \{return null;\}

package isol4649;

// Imports

import java.util.ArrayList;

public class tool_knurl extends knurl \{

// Constructors

public tool_knurl() \{\}

public tool_knurl(String_its_id, workpiece_its_workpiece, machining_operation[]

its_operations, axis2_placement_3d_feature_placement, turning_feature _base_feature,

partial_area_definition_partial_profile, toleranced_length_measure _tooth_depth, toleranced_length_measure_diametral_pitch, toleranced_length_measure _root_fillet, Integer_number_of_teeth, toleranced_length_measure_major_diameter,

toleranced_length_measure_nominal_diameter) \{\}

// Methods

public ArrayList get prop_names() \{return null;

public void set_attributes_from_part21_line(String part21line, ArrayList A) \{\} 
Appendix C

public ArrayList get_attributes() \{return null; \} \}

public ArrayList get_output() \{return null;\} 
Appendix D - Activity Diagrams

Activity Diagrams - Starting Header

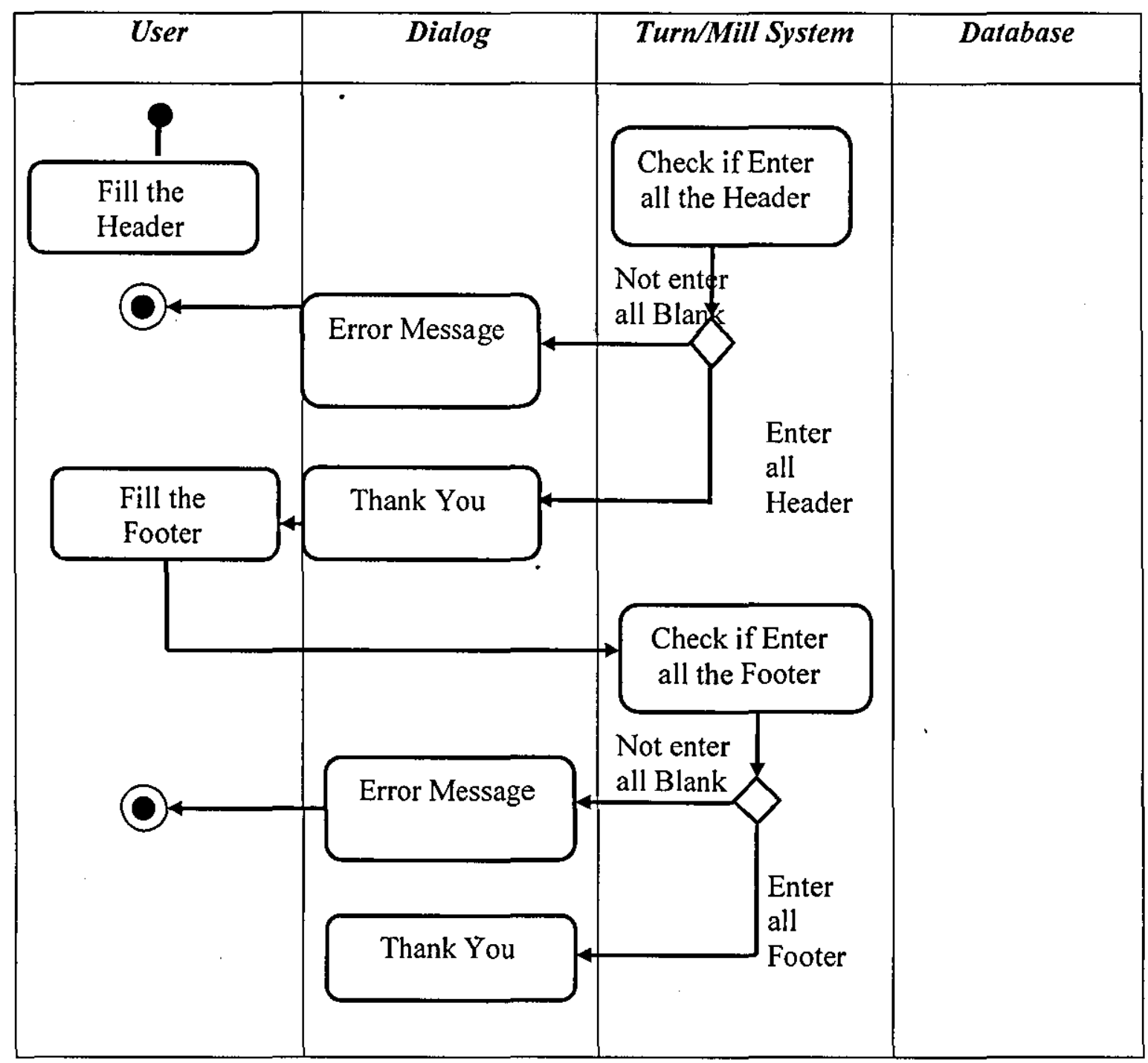


Appendix D

Activity Diagrams - Machining Parameter

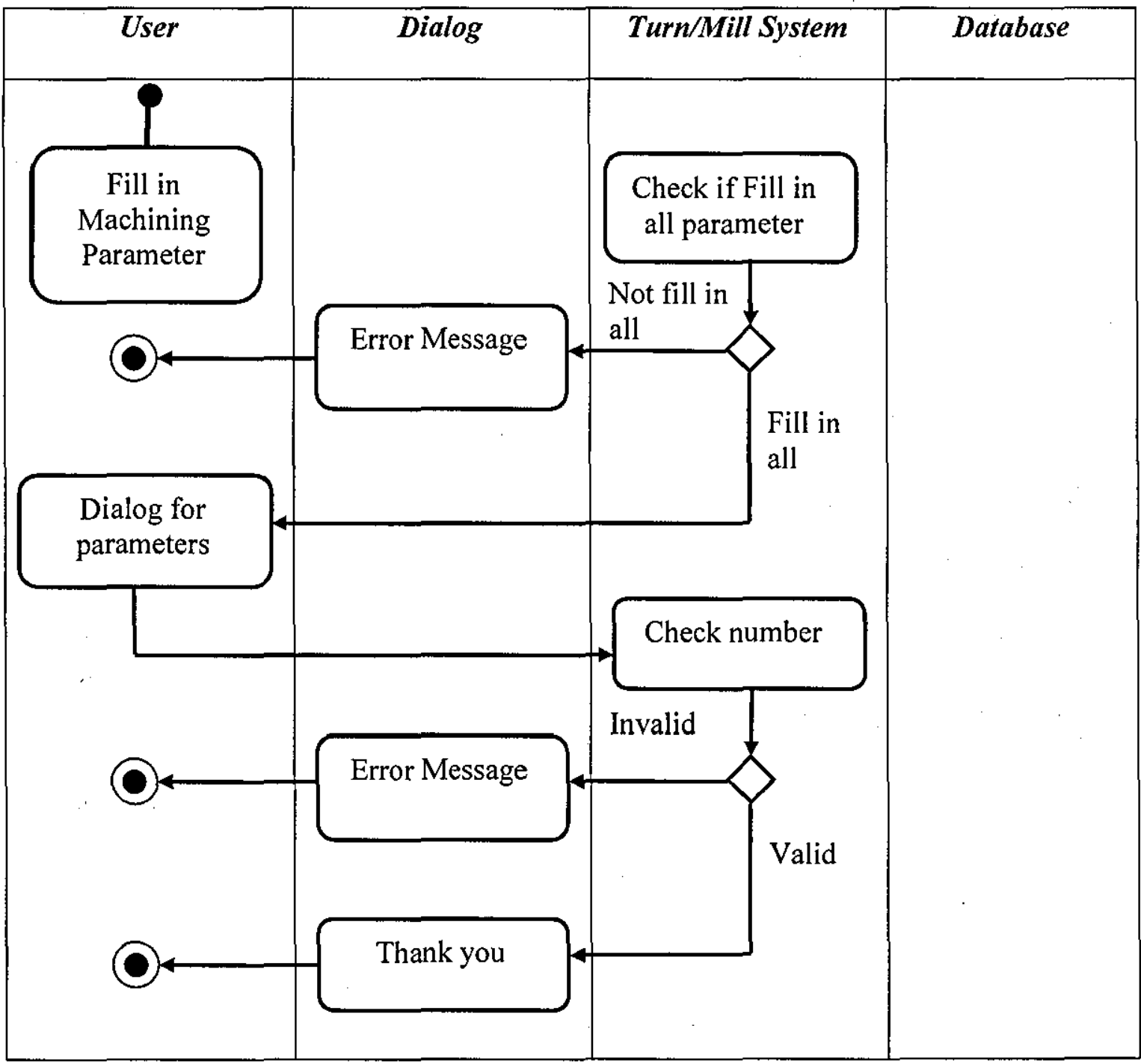


Appendix D

Activity Diagrams - New Feature

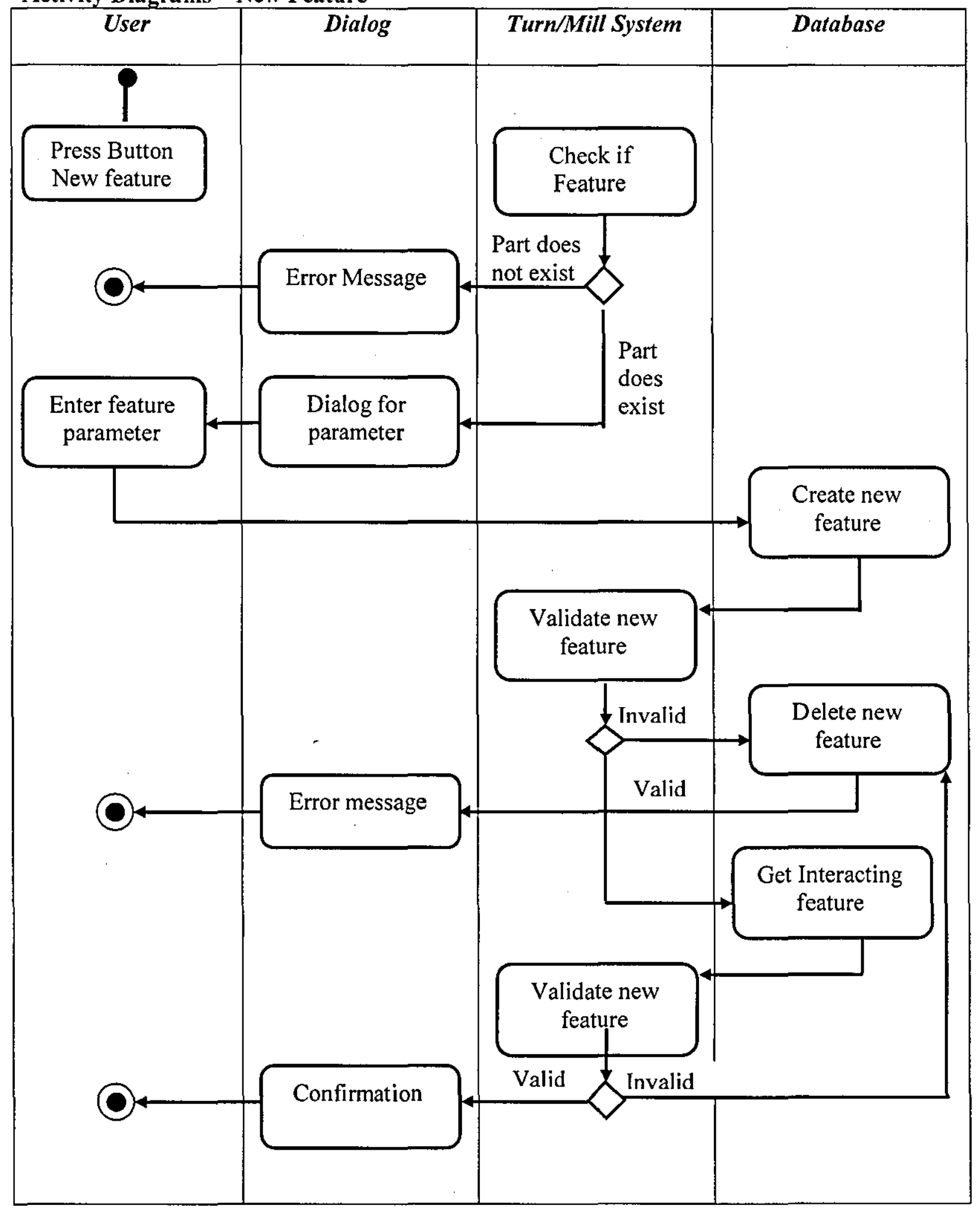


Appendix D

Activity Diagrams - STEP File

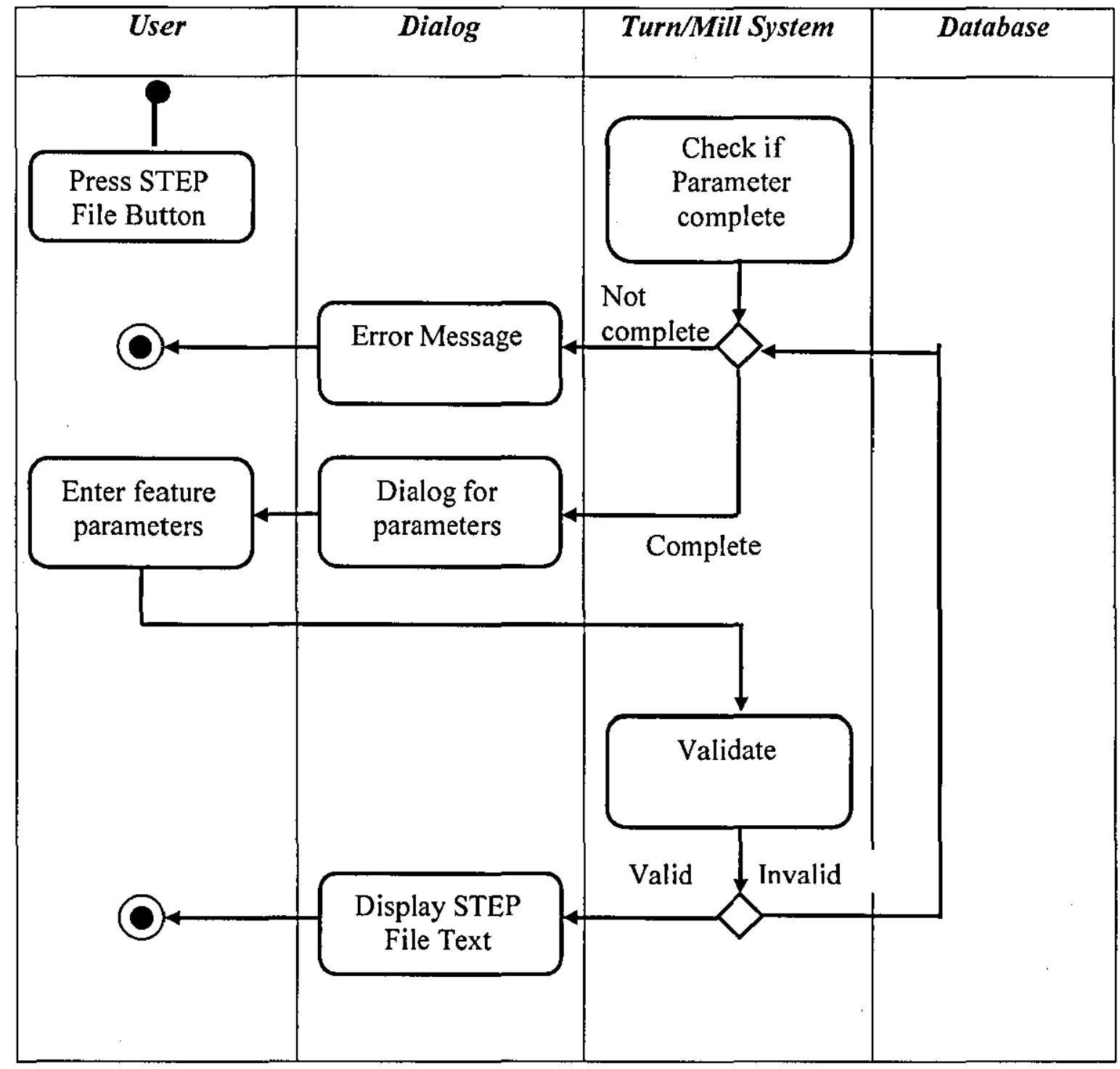




\section{Appendix E - Implementation Case Study Component 1}

\section{Implementation Case Study Component No 1}

The value of this research work is highlighted by the use of two case studies, one related to the representation of a standard turning component and the other, the representation of a turn-mill component machined with a turning centre. Both components have been designed using Unigraphics NX3. The operation planning for the two components requires different types of machining and has different demands on the number of machine axes, features, and the number of set-ups and level of complexity. In each case, the goal has been to make the product's processplanning more efficient, faster and if possible more secure.

The process for completed the case study component including pictorial views are shown in figures E1 - E8. The first component is a standard turning component based on ISO 14949 Part 12 and has been machined by a Haas Turning machine in Kolej Universiti Teknologi Tun Hussein Onn, Malaysia. DataPilot 3190 version 5.31 from Heidenhein was chosen to program this component because the geometry for the blank and the machined component is described with interactive graphics as shown. DataPilot with TURN PLUS allows the user to program the contour using the dimensions from the drawing in either Cartesian or polar coordinates and also in absolute or incremental form. With TURN PLUS the author can create NC programs in a very short time after entering the geometry for the blank and machined part. Having selected the material and clamping devices, TURN PLUS does everything else automatically. TURN PLUS selects the machining strategy, generates the work plan, selects the tools and cutting data, and generates the NC blocks both for turning operations as well as for face and lateral-surface machining.

The case study component based on ISO 14649 Part 12 (ISO 14649) has seven machining_features; 2 circular_face, 2 outer_diameter, 1 general_revolution, 1 groove, 1 cut_in. The final shape is machined by 2 setups; setup 1 is for right side features and setup 2 if for left side features. 


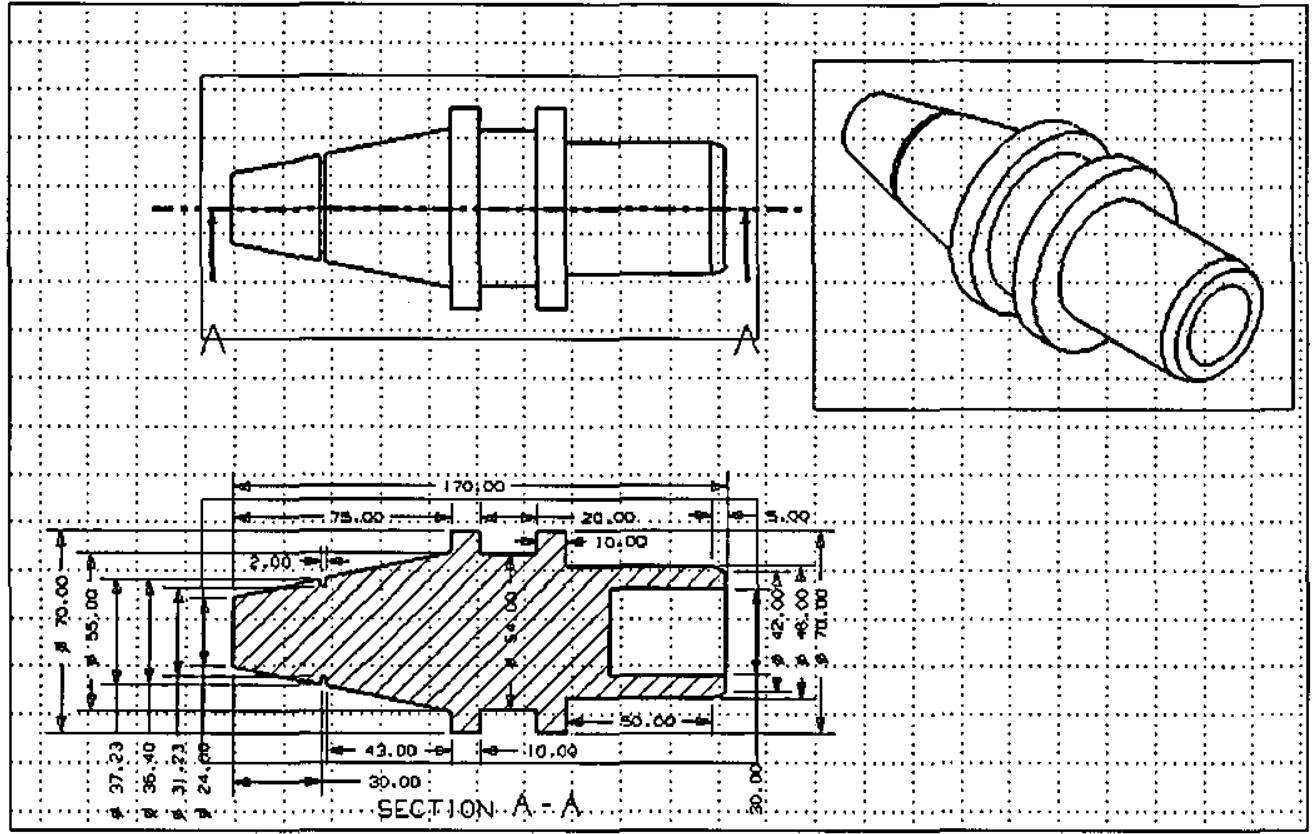

Figure E1: Drawing for Case Study Component

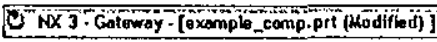

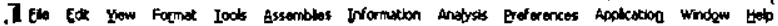

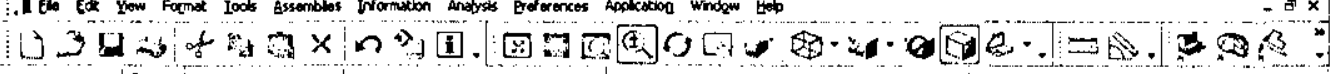

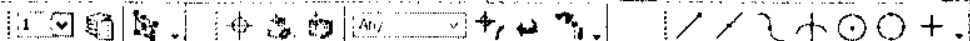

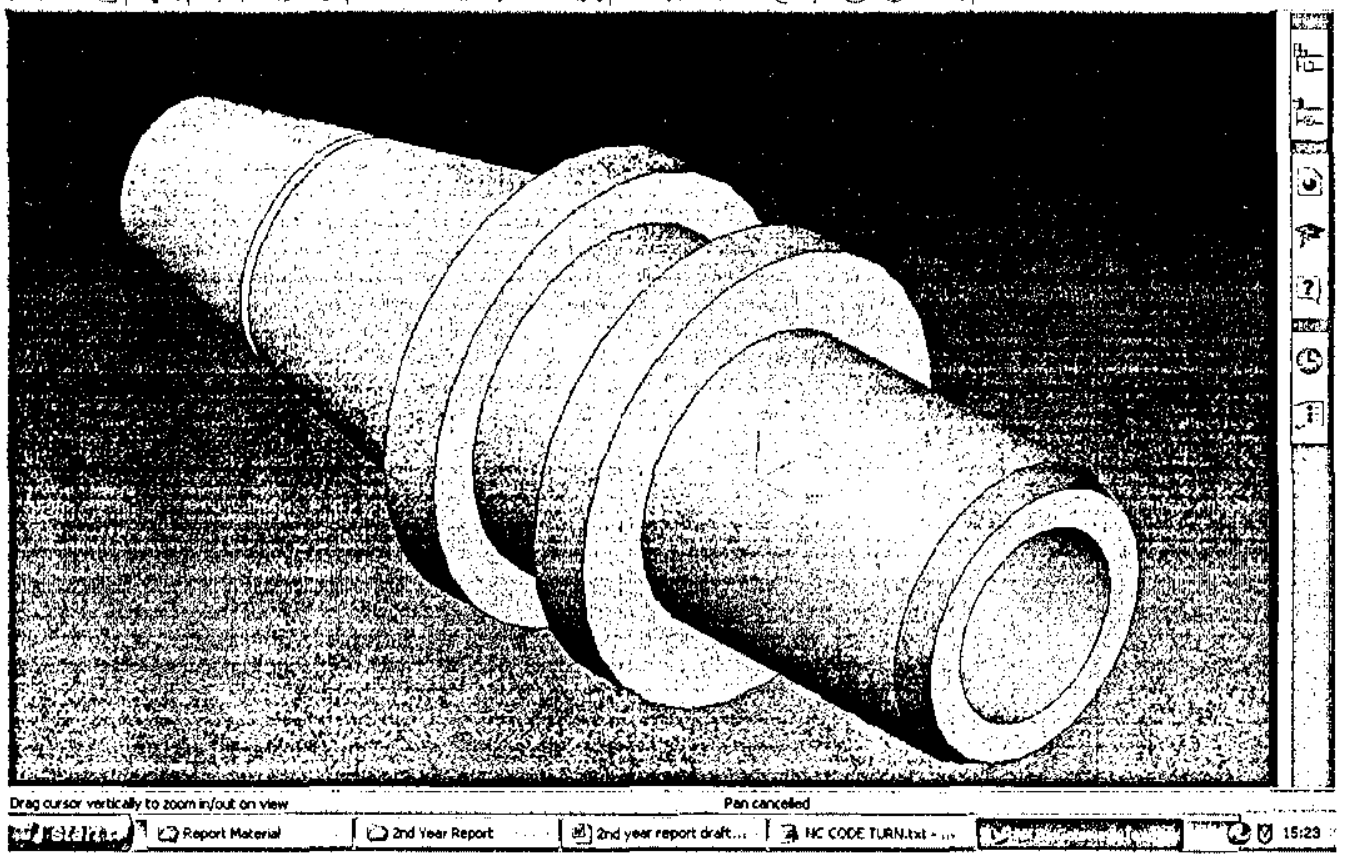

Figure E2: Unigraphic NX3 Modelling 


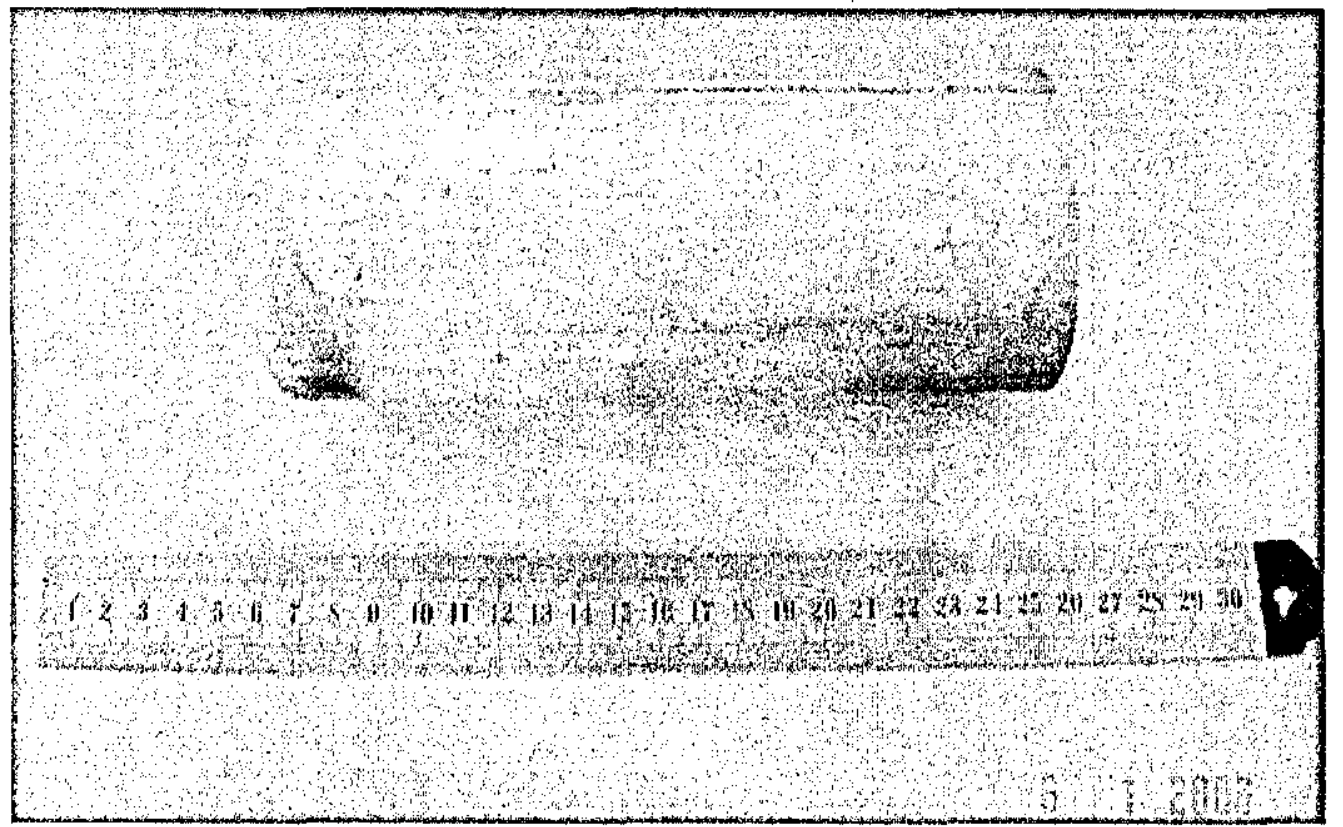

Figure E3: Material Aluminium

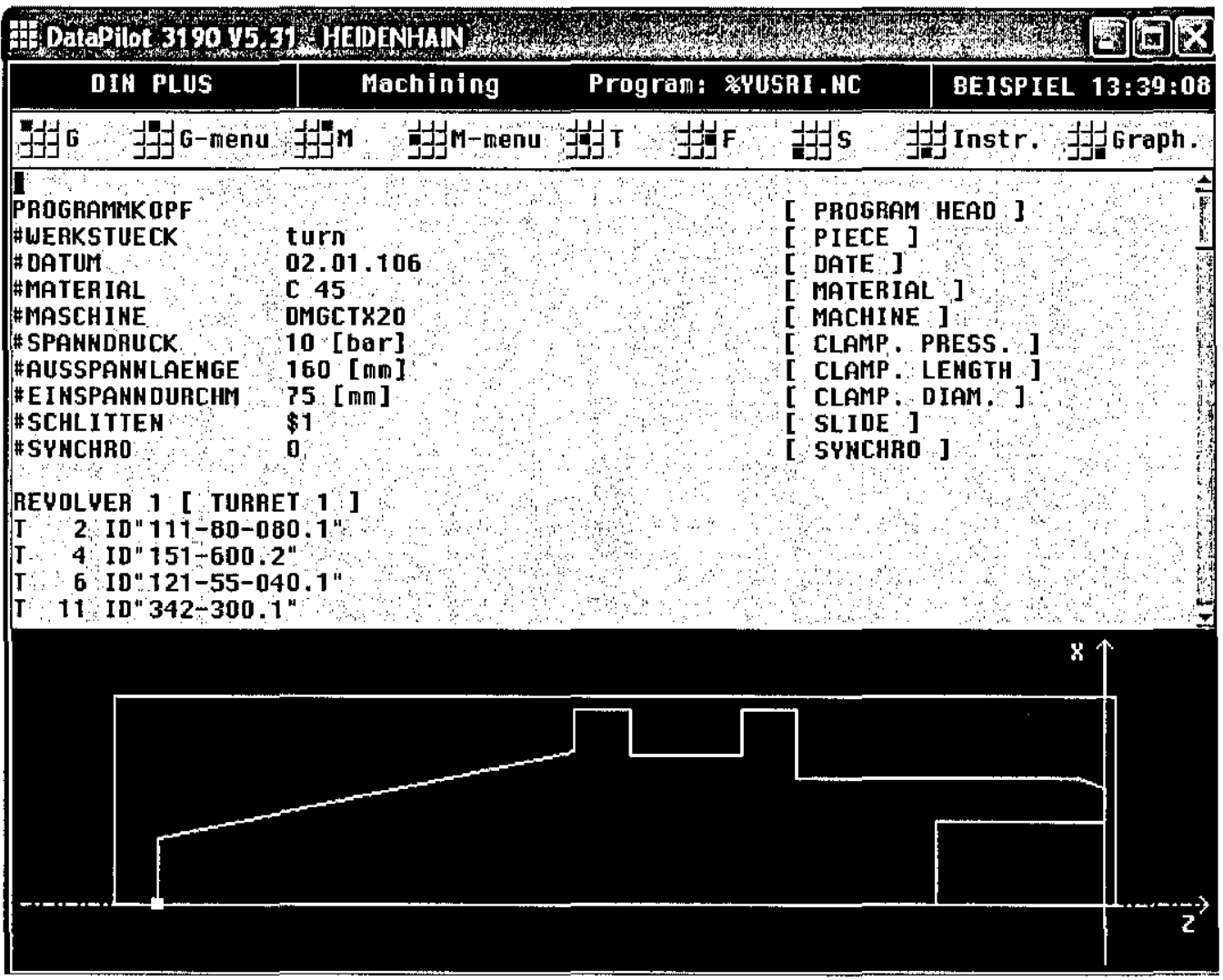

Figure E4: DataPilot Programming 


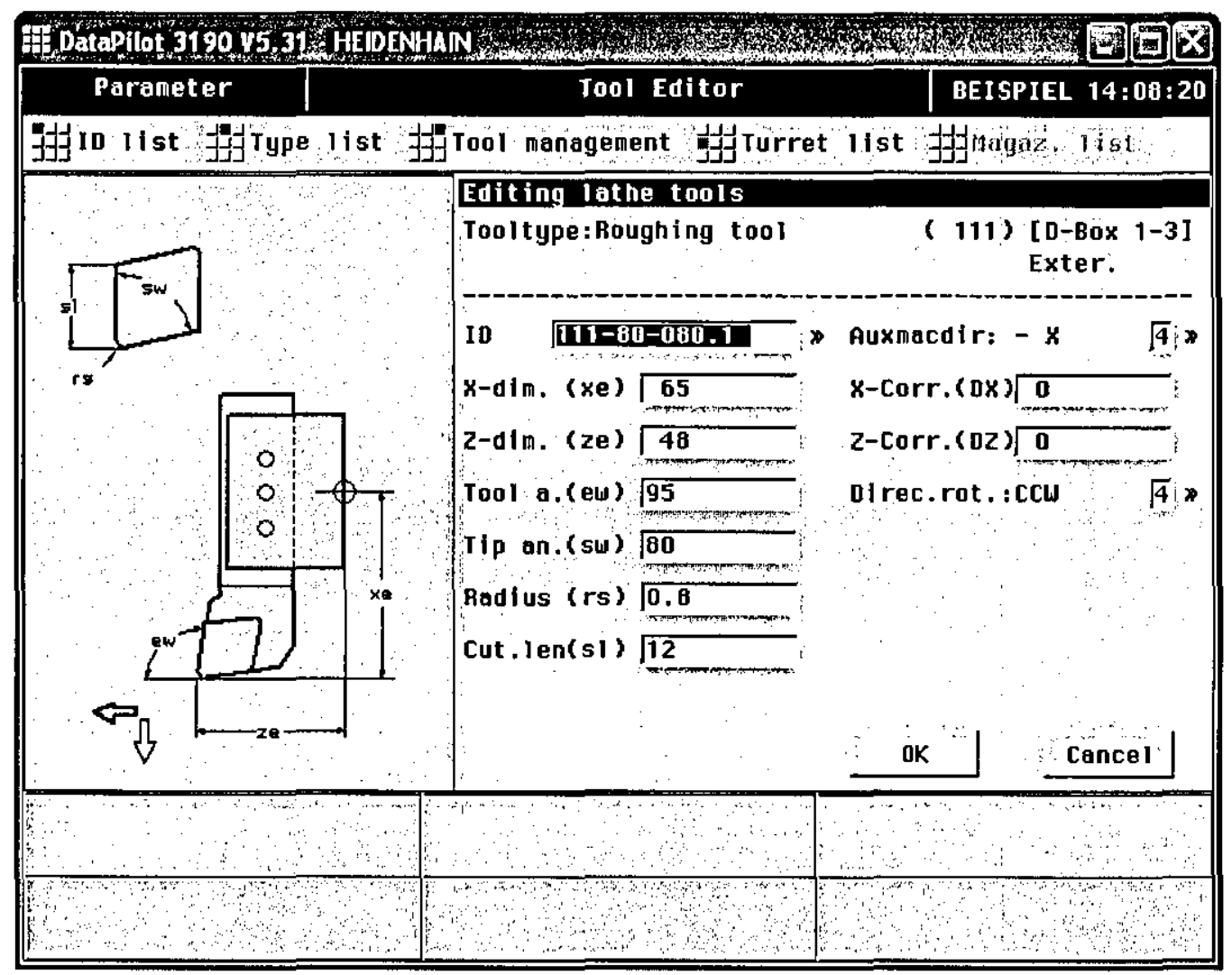

Figure E5: DataPilot Detail Tool Data

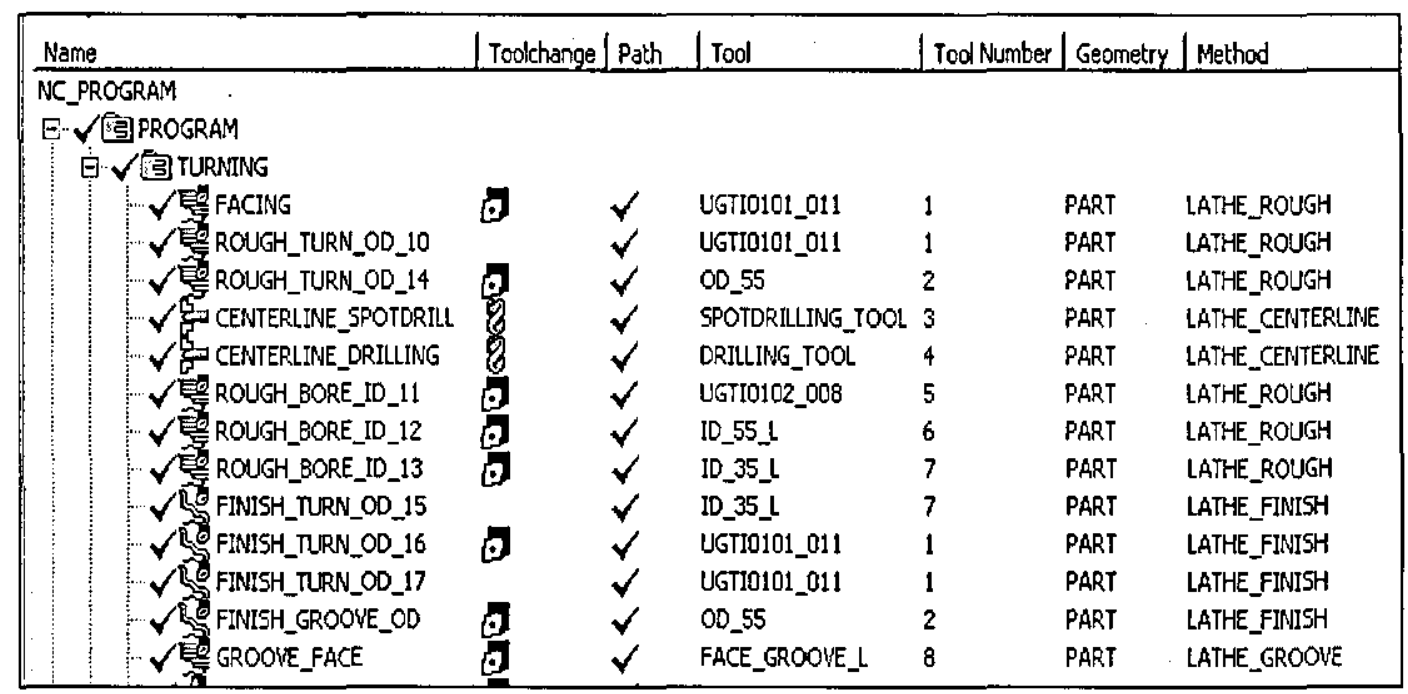

Figure E6: Unigraphic NX3 Operation Program Navigator 
Appendix E

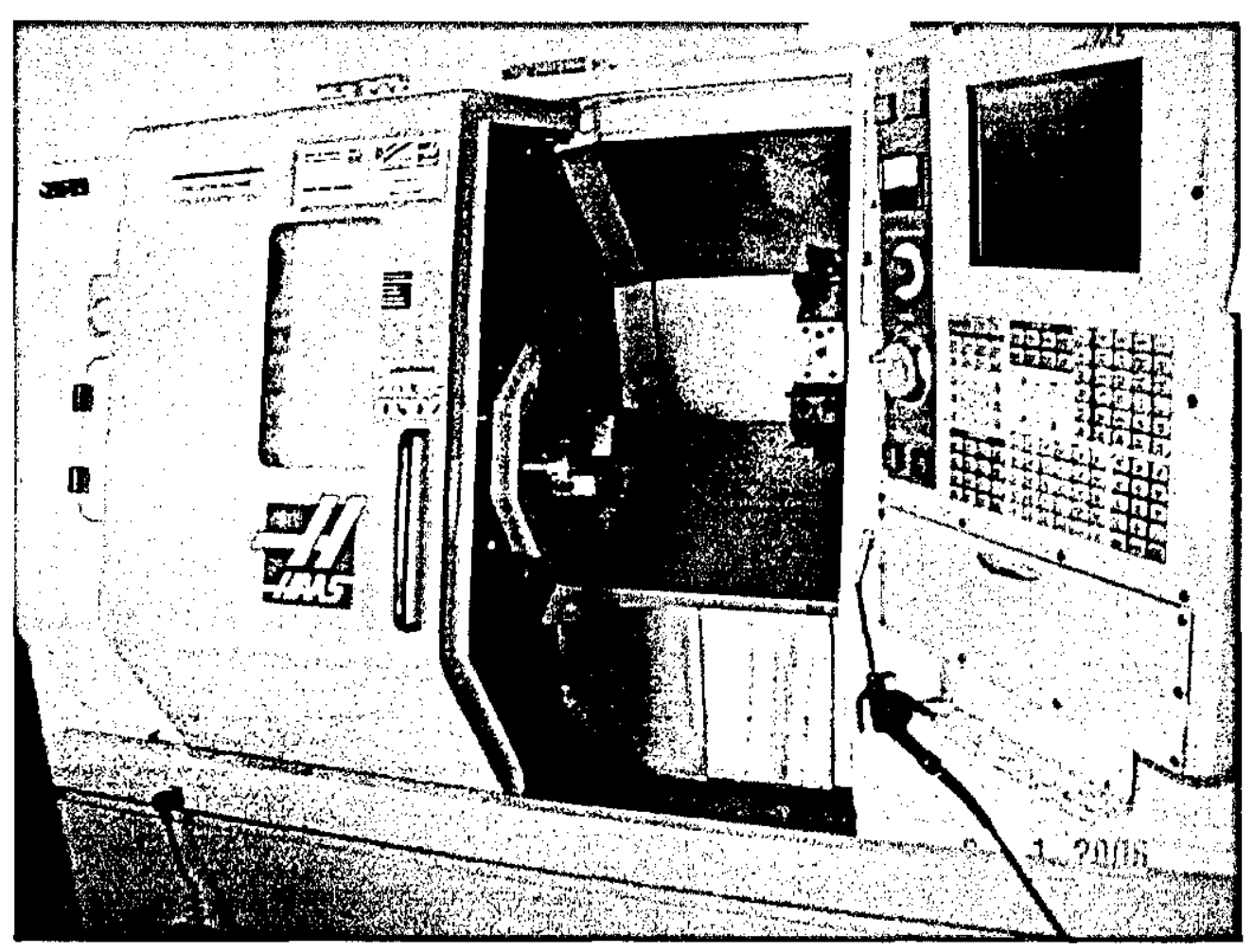

Figure E7: Haas CNC Turning Machine

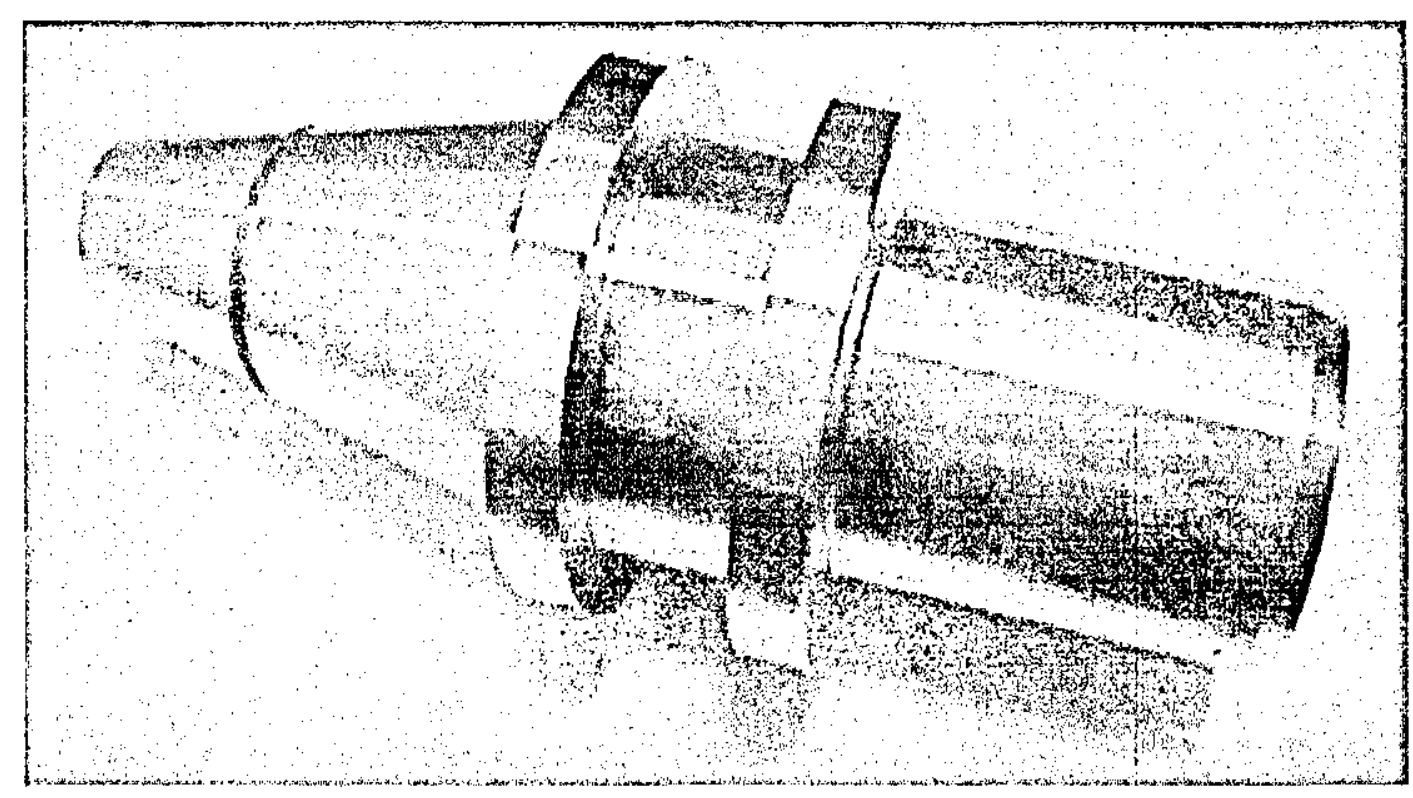

Figure E8: Final part 


\title{
Appendix F - ISO 14649 Physical File for Component 1
}

\author{
ISO-10303-21; \\ HEADER; \\ FILE_DESCRIPTION(('EXAMPLE OF NC PROGRAMME FOR TURNING: \\ COMPLEX DESIGN.'),'1'); \\ FILE_NAME('CASE STUDY COMPONENT NO 1'); \\ FILE_SCHEMA(); \\ ENDSEC; \\ DATA; \\ $\# 1=$ WORKPIECE('SIMPLE WORKPIECE',\#2,0.01,\$,\$,\$,()); \\ \#2=MATERIAL('ALUMINIUM',(\#3)); \\ \#3=PROPERTY_PARAMETER('E=200000N/M2'); \\ \#4=RIGHT_CIRCULAR_CYLINDER('WORKPIECE PIECE', \#5,175.0, 35.0); \\ $\# 5=A X I S 1$ PLACEMENT('WORKPIECE PIECE PLACEMENT',\#6,\#7); \\ \#6=CARTESIAN_POINT('WORKPIECE PIECE: LOCATION ',(0.000,0.000,0.000)); \\ $\# 7=$ DIRECTION(' AXIS ',(0.000,0.000,1.000)); \\ $\# 10=$ REVOLVED_FLAT('REVOLVED FLAT 1',\#1,(\#22,\#23),\#172,\#176,21.0,\#178); \\ $\# 11=$ REVOLVED_FLAT('REVOLVED FLAT 2',\#1,(\#31,\#32),\#183\#187,12.0,\#189); \\ $\# 12=$ GENERAL_REVOLUTION('GENERAL_REVOLUTION \\ $1 ', \# 1,(\# 20, \# 21), \# 194, \# 198,21.0, \# 200)$; \\ \#13=ROUND_HOLE('HOLE1 FLAT BOTTOM \\ '\#1,(\#26,\#27,\#28),\#207,\#215,\#216,\$,\#217); \\ \#14=OUTER_DIAMETER('OUTER_DIAMETER 1',\#1,(\#29,\#30), \\ $\# 218, \# 222, \# 223, \# 224)$; \\ $\# 15=$ GROOVE('GROOVE 1',\#1,(\#24,\#25), \#226,\#230,35.0,\#232); \\ $\# 16=$ GROOVE('CUT_IN',\#1,(\#33),\#236,\#240,18.4,\#242); \\ $\# 20=$ CONTOURING_ROUGH $(\$, \$$, ROUGH \\ GENERALREVOLUTION1',30.000,\$,\#280,\#61,\#60,\#130,\#130,\#131,0.5); \\ \#21=CONTOURING_FINISH(\$,\$,'FINISH GENERAL REVOLUTION \\ $1,30.000, \$, \# 280, \# 61, \# 60, \# 130, \# 130, \# 132,0.0)$; \\ \#22=FACING_ROUGH( $\$, \$, '$ ROUGH CIRCULAR FACE \\ $2 ', 50.000, \$, \# 280, \# 63, \# 60, \# 133, \# 134, \# 135,0.500)$; \\ \#23=FACING_FINISH(\$,\$,'FINISH CIRCULAR FACE \\ $2 ', 50.000, \$, \# 280, \# 63, \# 60, \# 133, \# 134, \# 136,0.0)$; \\ \#24=GROOVING_ROUGH $(\$, \$$, ROUGH GROOVE \\ $1 ', 30.000, \$, \# 285, \# 65, \# 60, \# 137, \# 137, \# 138, \$, 0.500)$; \\ \#25=GROOVING_FINISH(\$,\$,'FINISH GROOVE \\ $\left.1^{1}, 30.000, \$, \# 285, \# 65, \# 60, \# 137, \# 137, \# 139, \$, 0.0\right)$; \\ \#26= DRILLING(\$,\$,'DRILL HOLE1 $1,30.000, \$, \# 289, \# 66, \# 67, \$, \$, \$, \$, \$, \# 140)$; \\ $\# 27=$ REAMING $(\$, \$, ' R E A M$ HOLE $1,30.000, \$, \# 293, \# 69, \# 67, \$, \$, \$, \$, \$, \# 141,, T ., \$, \$)$; \\ \#29=CONTOURING_ROUGH(\$,\$,'ROUGH OUTER DIAMETER \\ $1,30.000, \$, \# 280, \# 61, \# 60, \# 130, \# 130, \# 131,0.5)$; \\ $\# 30=$ CONTOURING_FINISH(\$,\$,'FINISH OUTER_DIAMETER \\ $1 ', 30.000, \$, \# 280, \# 61, \# 60, \# 130, \# 130, \# 132,0.0)$; \\ \#31=FACING_ROUGH $(\$, \$$, ROUGH CIRCULAR FACE \\ $1,50.000, \$, \# 280, \# 63, \# 60, \# 133, \# 134, \# 135,0.500)$; \\ \#32=FACING_FINISH(\$,\$,'FINISH CIRCULAR FACE
}


ISO14649 Part 21 Physical File for Case Study Component No 1- (continued)

$1,50.000, \$, \# 280, \# 63, \# 60, \# 133, \# 134, \# 136,0.0)$;

$\# 33=$ CUTTING_IN $(\$, \$, ' C U T T I N G$ IN I',50.000,\$,\#297,\#70,\#60,\#142,\#142,\#143,\$,0.0);

\#34=PROJECT('TURNING EXAMPLE 1',\#35,(\#1),\$,\$,\$);

$\# 35=$ WORKPLAN('MAIN WORKPLAN',(\#36,\#37),\$,\#52,\$);

\#36=WORKPLAN('WORK PLAN FOR

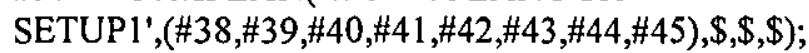

\#37=WORKPLAN('WORK PLAN FOR SETUP2',(\#47,\#48,\#49,\#50,\#51),\$,\#54,\$);

$\# 38=$ MACHINING_WORKINGSTEP('WS ROUGH CIRCULAR_FACE 2',\#56,\#11,\#22);

\#39=MACHINING_WORKINGSTEP('WS FINISH CIRCULAR_FACE 2',\#56,\#11,\#23);

\#40=MACHINING_WORKINGSTEP('WS ROUGH GENERAL_REVOLUTION

$1 ', \# 56, \# 12, \# 20$ );

\#41=MACHINING_WORKINGSTEP('WS FINISH GENERAL_REVOLUTION

$1 ', \# 56, \# 12, \# 21$;)

\#42=MACHINING_WORKINGSTEP('WS ROUGH GROOVE 1',\#56,\#15,\#24);

\#43=MACHINING_WORKINGSTEP('WS FINISH GROOVE 1',\#56,\#15,\#25);

\#44=MACHINING_WORKINGSTEP('WS DRILLING',\#56,\#13,\#26);

\#45=MACHINING_WORKINGSTEP('WS REAMING',\#56,\#13,\#27);

$\# 47=$ MACHINING_WORKINGSTEP('WS ROUGH CIRCULAR_FACE 1',\#56,\#10,\#30);

\#48=MACHINING_WORKINGSTEP('WS FINISH CIRCULAR_FACE 1',\#56,\#10,\#31);

\#49=MACHINING_WORKINGSTEP('WS ROUGH OUTER_DIÄMETER

$2 ', \# 56, \# 14, \# 28$;

\#50=MACHINING_WORKINGSTEP('WS FINISH OUTER_DIAMETER

$2 ', \# 56, \# 14, \# 29$ );

\#51=MACHINING_WORKINGSTEP('WS FINISH CUT_IN 1',\#56,\#16,\#32);

\#52=SETUP('SETUP 1',\#103,\#56,(\#53));

$\# 53=$ WORKPIECE_SETUP(,\#107,\$,\$,\$);

$\# 54=$ SETUP('SETUP 2',\#111,\#56,(\#55));

$\# 55=$ WORKPIECE_SETUP(,\#115,\$\$,\$);

\#56=PLANE('SECURITY PLANE',\#119);

$\# 60=$ TURNING_MACHINE_FUNCTIONS(.T.,\$,\$,(),.F.,\$,\$,(),\$,\$,\$);

$\# 61=$ TURNING_TECHNOLÖGY(\$,.TCP.,\#62,0.300,,F.,.F.,.F.,\$);

$\# 62=$ CONST_SPINDLE_SPEED $(500)$;

$\# 63=$ TURNING_TECHNOLOGY(\$,.TCP.,\#64,0.300,.F.,.F.,.F.,\$);

\#64=CONST_SPINDLE_SPEED(500);

$\# 65=$ TURNIN̄G_TECHNOLOGY(\$,.TCP.,\#66,0.300,.F.,.F.,.F.,\$);

\#66=CONST_SPINDLE_SPEED(200);

\#67= MILLIN̄G_MACHINE_FUNCTIONS(.T.,\$,\$,F.,\$,(), T.,\$,\$,());

$\# 66=$ MILLING_TECHNOLÖGY $(0.030$, TCP.,\$, 16.000,\$,.F.,.F.,.F.,\$);

$\# 69=$ MILLING_TECHNOLOGY $(0.030$, .TCP.,\$, 18.000,\$,.F.,.F.,.F.,\$);

$\# 70=$ TURNING_TECHNOLOGY(\$,.TCP.,\#71,0.300,.F.,.F.,F.,\$);

$\# 71=$ CONST_SPINDLE_SPEED $(100)$;

$\# 130=$ PLUNGE_RAMP $(\overline{\$}, 45.000)$;

$\# 131=U N I D I R E C T I O N A L$ TURNING $(\$, \$,(3.000), \$, \$, \$, \$, \$, 2.000, \$, \$)$;

\#132=UNIDIRECTIONAL_TURNING $(\$, \$,(0.500), \$, \$, \$, \$, \$, \$, \$)$;

$\# 133=$ PLUNGE_RAMP $(\$, 30.000)$;

$\# 134=$ PLUNGE_RAMP $(\$, 40.000)$;

$\# 131=$ UNIDIRECTIONAL_TURNING(\$,.T.,(3.000),\$,\$,\$,\$,\$,2.000,\$,\$);

$\# 132=$ UNIDIRECTIONAL_TURNING $(\$, F .,(0.500), \$, \$, \$, \$, \$, \$, \$, \$)$; 
ISO14649 Part 21 Physical File for Case Study Component No 1- (continued)

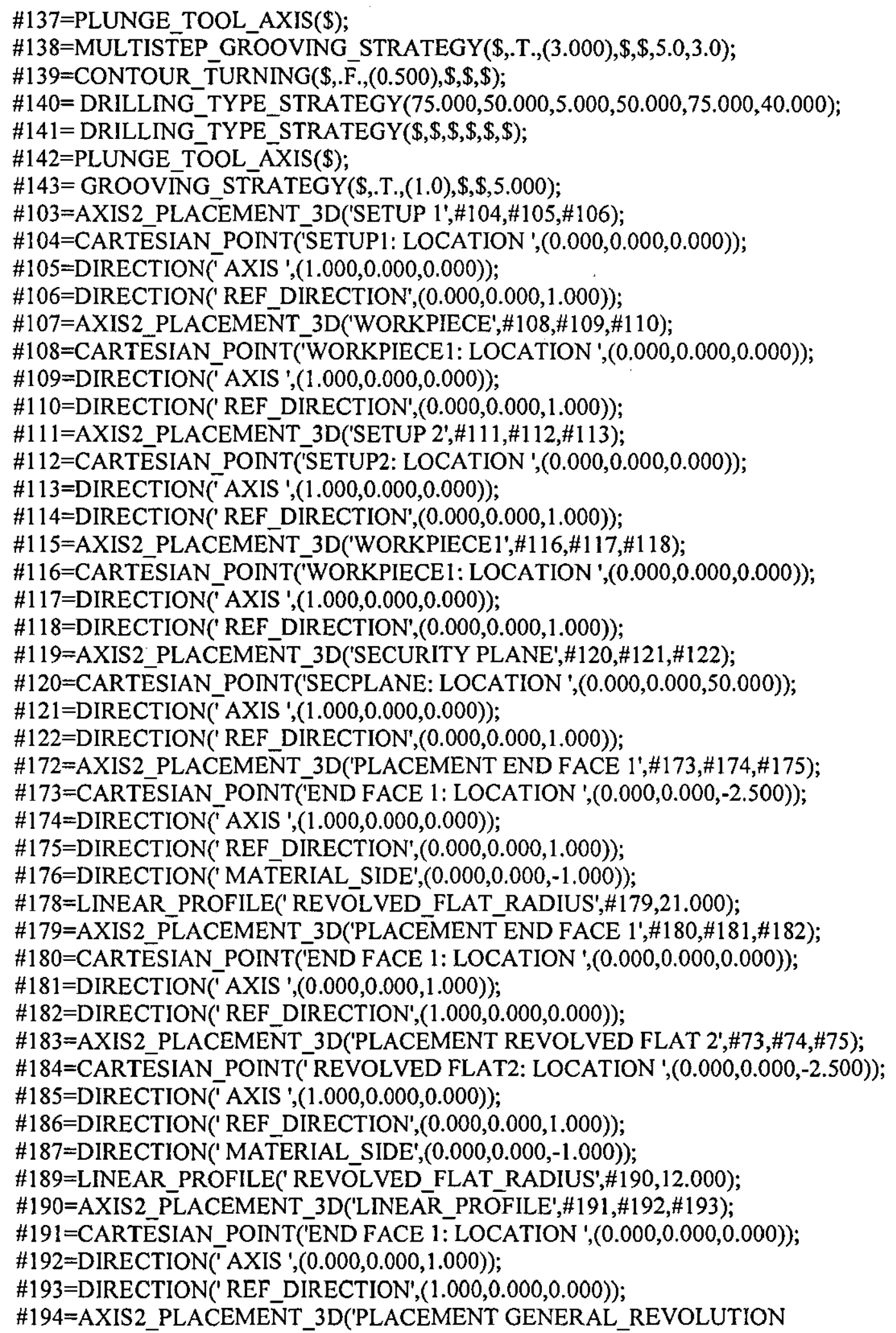


ISO14649 Part 21 Physical File for Case Study Component No 1- (continued)

1',\#195,\#196,\#197);

\#195=CARTESIAN_POINT(' GENERAL_REVOLUTION : LOCATION ',(0.000,0.000, 2.500));

\#196=DIRECTION(' AXIS ',(1.000,0.000,0.000));

\#197=DIRECTION(' REF_DIRECTION',(0.000,0.000,1.000));

\#198=DIRECTION(' MATERIAL_SIDE',(-1.000,0.000,0.000));

$\# 200=$ GENERAL_PROFILE $(\$$, $\# 201)$;

\#201=POLYLINE(",(\#202,\#203,\#204,\#205,\#206));

\#202=CARTESIAN_POINT(", $(21.000,0.000,0.000)$ );

\#203=CARTESIAN_POINT(",(23.000,0.000, 5.000));

\#204=CARTESIAN_POINT(",(23.000,0.000, 55.000));

\#205=CARTESIAN_POINT(",(35.000,0.000, 55.000));

\#206=CARTESIAN_POINT(",(35.000,0.000, 95.000));

\#207= AXIS2_PLACEMENT_3D('HOLE3',\#208,\#209,\#210);

$\# 208=$ DIRECTION $(",(0 ., 0 ., 1 . \overline{)})$;

\#209= DIRECTION(",(1.,0.,0.));

$\# 210=$ CARTESIAN_POINT(",(0.,0.,0.));

$\# 211=$ AXIS2_PLACEMENT_3D(",\#212,\#213,\#214);

\#212=CARTESIAN_POINT(", $(0.000,0.000,-40.000))$;

\#213=DIRECTION(", $(0.000000,0.000000,1.000000))$;

\#214=DIRECTION(",(1.000000,0.000000,0.000000));

\#215=PLANE(",\#211);

\#216= TOLERANCED_LENGTH_MEASURE(15.0,\#251);

$\# 217=$ FLAT_HOLE_BOTTOM();

\#218=AXIS2_PLACEMENT_3D('PLACEMENT OUTER_DIAMETER

$1 ;, \# 219, \# 220, \# 221$ );

\#219=CARTESIAN_POINT(' OUTER_DIAMETER 2: LOCATION ',(0.000,0.000, -

77.500));

\#220=DIRECTION(' AXIS ',(1.000,0.000,0.000));

\#221=DIRECTION(' REF_DIRECTION',(0.000,0.000,1.000));

\#222=TOLERANCED_LENGTH_MEASURE(56.000,\#251);

\#223=TOLERANCED_LENGTH_MEASURE(75.000,\#251);

\#224=DIAMETER_TAPER;

\#225=TOLERANCED_LENGTH_MEASURE(24.000,\#251);

\#226=AXIS2_PLACEMENT_3D('PLACEMENT GROOVE 1',\#227,\#228,\#229);

\#227=CARTESIAN_POINT(' GROOVE 1: LOCATION ', $(0.000,0.000,-67.500)$ );

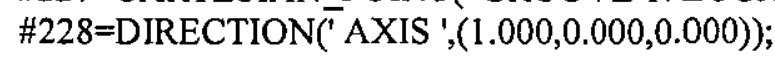

\#229=DIRECTION(' REF_DIRECTION',(0.000,0.000,1.000));

\#230=DIRECTION(' MATERIAL_SIDE',(-1.000,0.000,0.000));

\#232=SQUARE_U_PROFILE $($ \#234,0,\#235,0);

$\# 233=$ TOLERAN $\bar{N} \bar{C} D \_L E N G T H \_M E A S U R E(20.000$,\#251);

\#234=TOLERANCED_LENGTH_MEASURE $(0.000$,\#251);

\#235=TOLERANCED_LENGTH_MEASURE $(0.000$,\#251);

\#236=AXIS2_PLACEMENT_3D('PLACEMENT CUT_IN 1',\#237,\#238,\#239);

\#237=CARTESIAN_POINT(' CUT_IN 1: LOCATION ', $(0.000,0.000,-32.500))$;

$\# 238=$ DIRECTION(' AXIS ',(1.000,0.000,0.000));

\#239=DIRECTION(' REF_DIRECTION',(0.000,0.000,1.000));

\#240=DIRECTION(' MATERIAL_SIDE',(-1.000,0.000,0.000)); 
ISO14649 Part 21 Physical File for Case Study Component No 1- (continued)

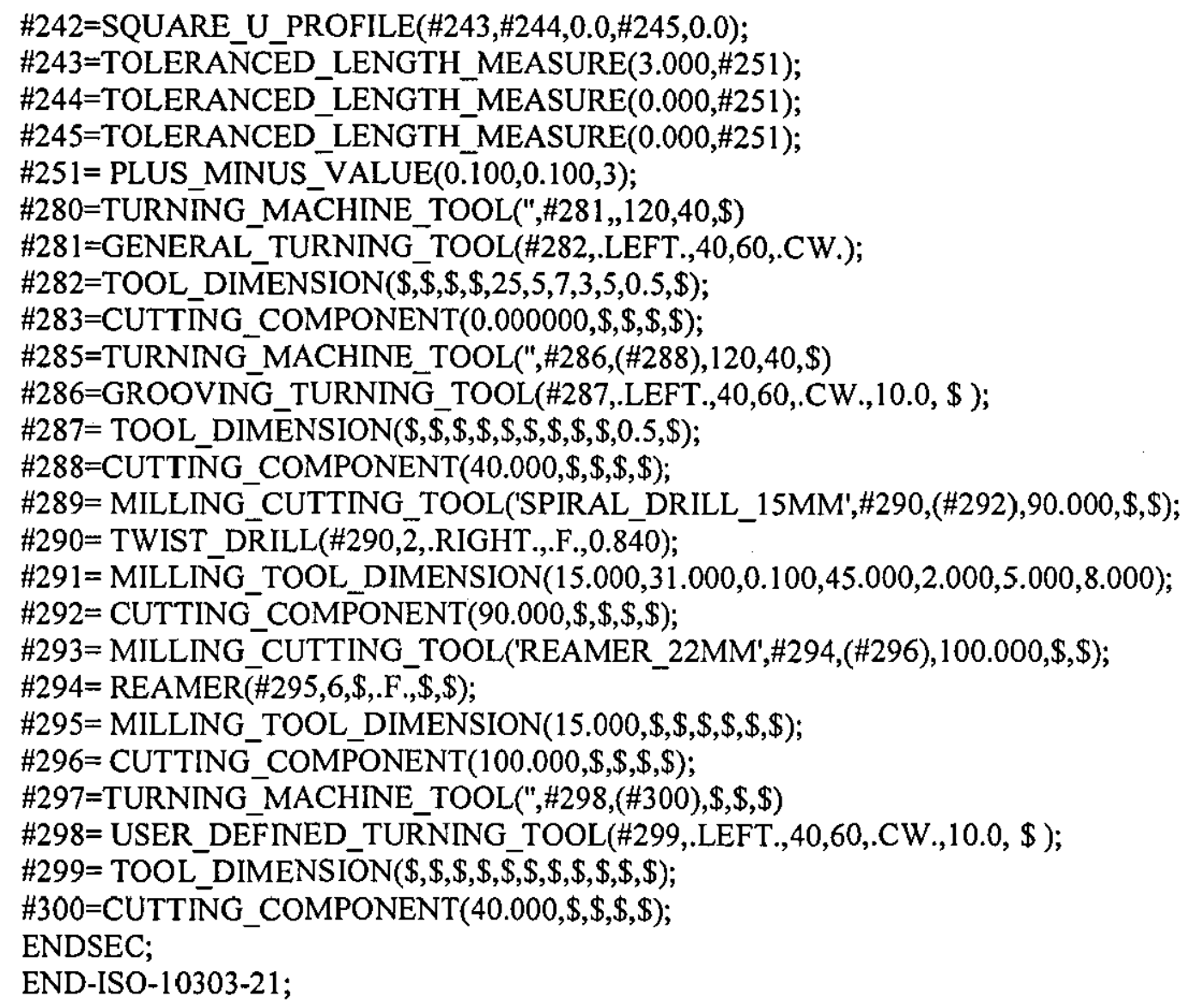




\section{Appendix G - G\&M Code for Case Study Component 1}

\%yusri.nc

PROGRAMMKOPF

\#MATERIAL C 45

\#MASCHINE DMGCTX20

\#SCHLITTEN \$1

\#SYNCHRO 0

\#WERKSTUECK turn

\#FIRMA KUITTHO

\#AUTOR Yusri

\#DATUM 02.01.106

\#EINSPANNDURCHM 75

[ PROGRAM HEAD ]

[MATERIAL ]

[ MACHINE ]

[ SLIDE ]

[SYNCHRO]

[ PIECE ]

[DEPT. ]

[AUTHOR ]

[ DATE ]

\#AUSSPANNLAENGE 160

[ CLAMP. DIAM. ]

\#SPANNDRUCK 10

[ CLAMP. LENGTH ]

[ CLAMP. PRESS. ]

REVOLVER 1 [ TURRET 1 ]

T2 ID"111-80-080.1"

T4 ID"151-600.2"

T6 ID"121-55-040.1"

T11 ID"342-300.1"

SPANNMITTEL 1 [ CHUCKING EQUIPMENT 1]

[ Zero point displacement Z348 ]

H1 ID"KH250"

H2 ID"HB240" X75 Q1.

ROHTEIL [ BLANK ]

N1 G20 X75 Z180 K2

FERTIGTEIL [ FINISHED PART ]

N2 G0 X0 Z-170

N3 G1 Z-30

N4 G1 X30

N5 G1 Z0

N6 G1 X42

N7 G1 X46 Z-5

N8 G1 Z-55

N9 G1 X70

N10 G1 Z-65

N11 G1 X54

N12 G1 Z-85

N13 G1 X70

N14 G1 Z-95

N15 G1 X55

N16 G1 X24 Z-170

N17 G1 X0 
G\&M Code for Case Study Component No 1 (continued)

BEARBEITUNG [ MACHINING ]

[ Centr.predrilling - Outside - Centric - Front face ]

N18 G65 H1 X0 Z-218 D1

N19 G65 H2 X75 Z-218 D1 Q1

N20 G14 Q0

N21 G26 S2000

N22 G97 S1000 M4

N23 T11

N24 G95 F0.2

N25 M108

N26 M107

$\mathrm{N} 27 \mathrm{G} 0 \mathrm{Z5}$

$\mathrm{N} 28 \mathrm{G} 0 \mathrm{X} 0$

$\mathrm{N} 29 \mathrm{G} 147 \mathrm{~K} 2$

N30 G74 Z-30 P75 I8 J30 E0 K0

N31 G14 Q0

N32 M109

[ Roughing - Transversal - Outside - Front face ]

N33 T2

N34 G96 S220 G95 F0.2 M4

N35 M108

N36 M107

N37 G0 X81 Z5

N38 G47 P3

N39 G820 NS6 NE6 P1 I1 K0.3 E0 Z-156 A90 W270 Q2 V3 D4

N40 G0 X48

N41 G0 Z5

N42 M109

[ Roughing - Longitudinal - Outside ]

N43 G95 F0.45

N44 M108

N45 M107

N46 G0 X81 Z3.3

$\mathrm{N} 47 \mathrm{G} 47 \mathrm{P} 3$

N48 G810 NS7 NE14 P2 I1 K0.3 E0 Z-156 A0 W180 Q2 V3 D4

N49 G0 Z3.3

N50 G0 X81

N51 G14 Q0

N52 M109

[ Contour cutting - Outside ]

N53 T4

N54 G96 S180 G95 F0.25 M4

N55 M108

N56 M107

N57 G0 Z-83.5

N58 G0 X77

N59 G47 P2

N60 G860 NS11 NE13 I0.4 K0.3 Z-156 V3 H0

N61 G0 X77 


\section{G\&M Code for Case Study Component No 1 (continued)}

N62 G0 Z-83.5

N63 G0 X250 Z3

N64 G14 Q0

N65 M109

[ Finishing - Contourparallel - Outside ]

N66 T6

N67 G96 S280 G95 F0.2 M4

N68 M108

N69 M 107

N70 G0 X36 Z3

N71 G47 P2

N72 G890 NS6 NE10 E0.2 V3 H4 Z-155 D1

N73 G0 X70 Z-82.6

N74 G1 Z-85

N75 M109

[ Finishing - Contourparallel - Outside ]

N76 M108

N77 M107

N78 G47 P2

N79 G890 NS14 E0.2 V3 Q3 H0 Z-155 D1 I76 K-92

N80 G14 Q0

N81 M9

N82 M30

ENDE [ END ] 


\section{Appendix H - Operation Plan for Case Study Component 2}

\begin{tabular}{|c|c|c|c|c|c|c|}
\hline \multicolumn{7}{|c|}{$\begin{array}{l}\text { Operation Plan for Component } 2 \\
\text { Material : Aluminium }\end{array}$} \\
\hline $\begin{array}{l}\text { Op. } \\
\text { No }\end{array}$ & $\begin{array}{l}\text { Op. } \\
\text { Type }\end{array}$ & Op. Description & $\begin{array}{l}\text { Tool } \\
\text { No. }\end{array}$ & $\begin{array}{c}\text { Tool } \\
\text { description }\end{array}$ & $\begin{array}{c}\text { Spindle } \\
\text { speed }\end{array}$ & $\begin{array}{c}\text { Cutting } \\
\text { feed }\end{array}$ \\
\hline 1 & Turn & Face the billet & $\mathrm{T} 1$ & Facing tool & 3600 & 0.2 \\
\hline 2 & Turn & $\begin{array}{l}\text { Rough the external } \\
\text { profile }\end{array}$ & $\mathrm{T} 2$ & $\begin{array}{l}\text { External } \\
\text { turning tool }\end{array}$ & 3600 & 0.2 \\
\hline 3 & Turn & $\begin{array}{l}\text { Finish external } \\
\text { profile }\end{array}$ & T3 & $\begin{array}{l}\text { External } \\
\text { turning tool }\end{array}$ & 3600 & 0.1 \\
\hline 4 & Turn & $\begin{array}{l}\text { Rough the internal } \\
\text { profile }\end{array}$ & $\mathrm{T} 2$ & $\begin{array}{l}\text { Internal } \\
\text { turning tool }\end{array}$ & 3600 & 0.2 \\
\hline 5 & Turn & $\begin{array}{l}\text { Finish the internal } \\
\text { profile }\end{array}$ & T3 & $\begin{array}{l}\text { Internal } \\
\text { turning tool }\end{array}$ & 3600 & 0.1 \\
\hline 6 & Turn & $\begin{array}{l}\text { Rough external } \\
\text { grooving }\end{array}$ & $\mathrm{T} 2$ & $\begin{array}{l}2 \mathrm{~mm} \text { External } \\
\text { grooving tool }\end{array}$ & 3600 & 0.2 \\
\hline 7 & Turn & $\begin{array}{l}\text { Finish external } \\
\text { grooving }\end{array}$ & $\mathrm{T} 3$ & $\begin{array}{l}2 \mathrm{~mm} \text { External } \\
\text { grooving tool }\end{array}$ & 3600 & 0.1 \\
\hline 7 & Mill & Mill slot & $\mathrm{T} 4$ & $\begin{array}{l}10 \mathrm{~mm} \text { End } \\
\text { mill }\end{array}$ & 3600 & 0.2 \\
\hline 8 & Turn & Part off & T5 & $\begin{array}{l}3 \mathrm{~mm} \text { parting } \\
\text { off tool }\end{array}$ & 3600 & 0.05 \\
\hline 9 & Turn & Workpiece transfer & & & 3600 & \\
\hline 10 & Turn & Rough facing & $\mathrm{T} 2$ & Facing tool & 3600 & 0.2 \\
\hline
\end{tabular}


Operation Plan for Component 2 (continued)

Material : Aluminium

\begin{tabular}{|c|c|c|c|c|c|c|}
\hline $\begin{array}{l}\text { Op. } \\
\text { No }\end{array}$ & $\begin{array}{l}\text { Op. } \\
\text { Type }\end{array}$ & Op. Description & $\begin{array}{l}\text { Tool } \\
\text { No. }\end{array}$ & $\begin{array}{c}\text { Tool } \\
\text { description }\end{array}$ & $\begin{array}{c}\text { Spindle } \\
\text { speed }\end{array}$ & $\begin{array}{c}\text { Cutting } \\
\text { feed }\end{array}$ \\
\hline 11 & Turn & $\begin{array}{l}\text { Rough external } \\
\text { profile }\end{array}$ & $\mathrm{T} 2$ & $\begin{array}{l}\text { External } \\
\text { turning tool }\end{array}$ & 3600 & 0.2 \\
\hline 12 & Turn & $\begin{array}{l}\text { Finish external } \\
\text { profile }\end{array}$ & T3 & $\begin{array}{l}\text { External } \\
\text { turning tool }\end{array}$ & 3600 & 0.1 \\
\hline 13 & Drill & Drilling & T6 & Drilling tool & 3600 & 0.2 \\
\hline 14 & Turn & $\begin{array}{l}\text { Rough internal } \\
\text { profile }\end{array}$ & $\mathrm{T} 2$ & $\begin{array}{l}\text { External } \\
\text { turning tool }\end{array}$ & 3600 & 0.2 \\
\hline 15 & Turn & $\begin{array}{l}\text { Finish internal } \\
\text { profile }\end{array}$ & $\mathrm{T} 3$ & $\begin{array}{l}\text { External } \\
\text { turning tool }\end{array}$ & 3600 & 0.1 \\
\hline 16 & Mill & Drill holes in face & T7 & $\begin{array}{l}3 \mathrm{~mm} \text { standard } \\
\text { drilling tool }\end{array}$ & 3600 & 0.2 \\
\hline
\end{tabular}


